\title{
A Telescoped Process to Manufacture 6,6,6- Trifluorofucose via Diastereoselective Transfer Hydrogenation: A Scalable Access to an Inhibitor of Fucosylation Utilized in Monoclonal Antibody Production
}

\section{Supporting Information}

Michal M. Achmatowicz, John G. Allen, Matthew M. Bio, Michael D. Bartberger, Christopher J. Borths, John T. Colyer, Richard D. Crockett, Tsang-Lin Hwang, Jan. N. Koek, Stephen A. Osgood, Scott W. Roberts, Aleksander Swietlow, Oliver R. Thiel, and Seb Caille*

Table of Contents:

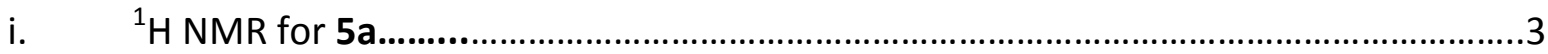

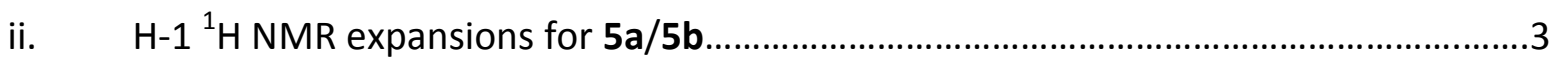

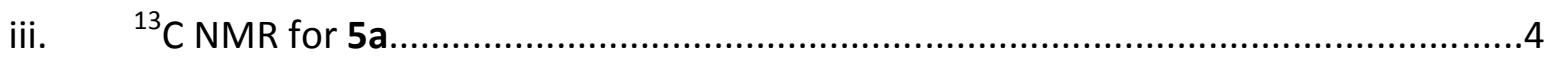

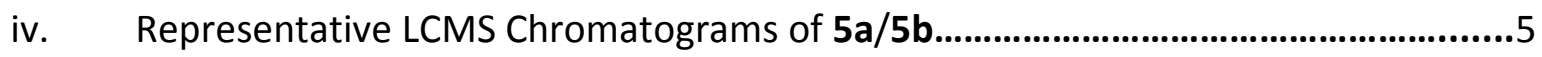

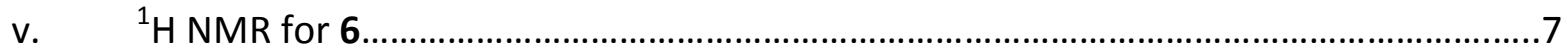

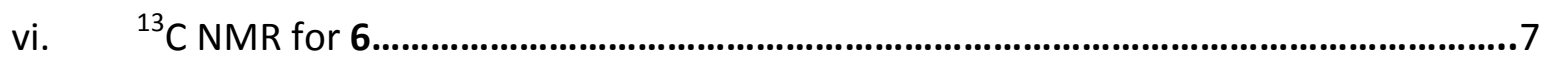

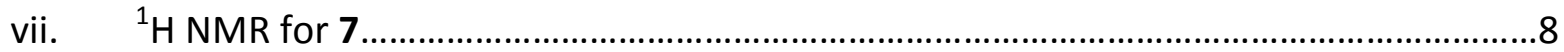

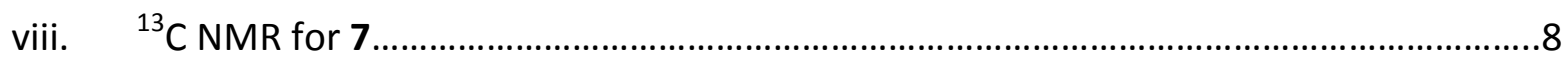

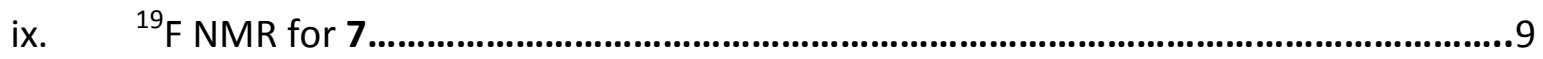

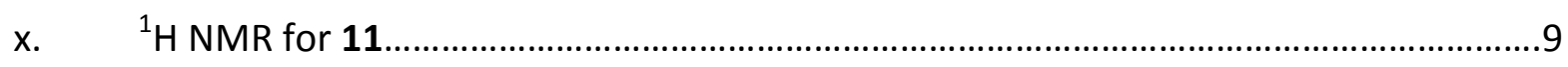

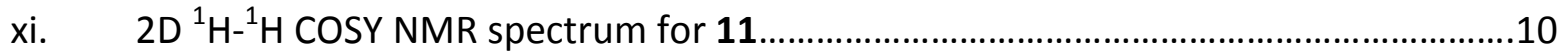

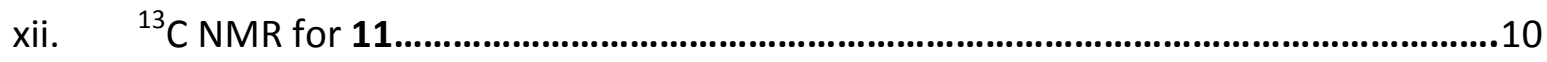

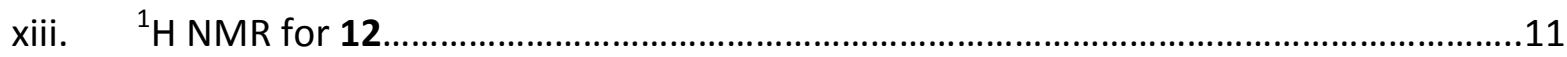




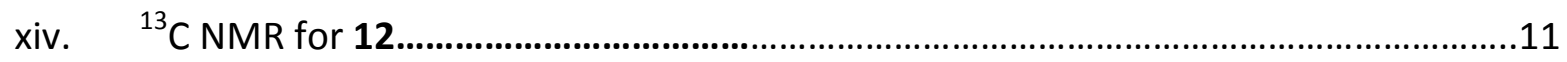

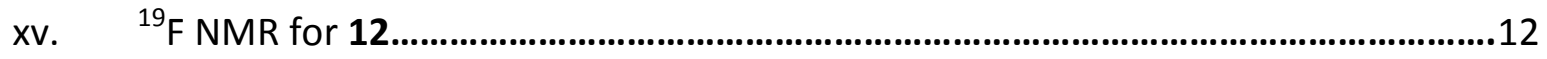

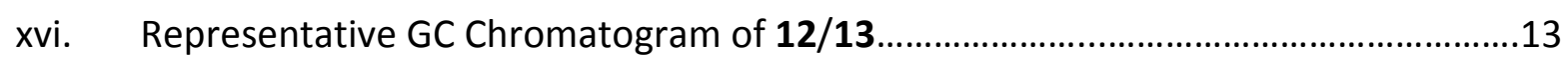

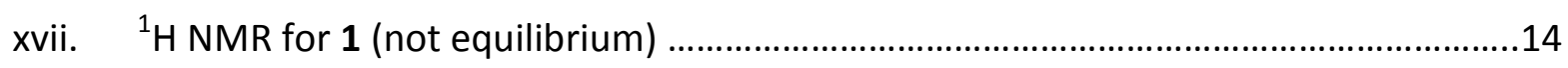

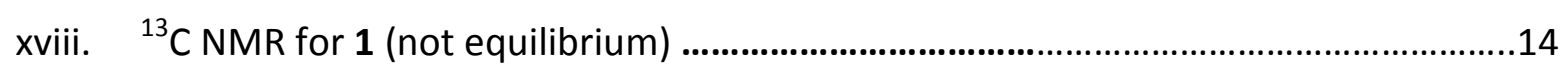

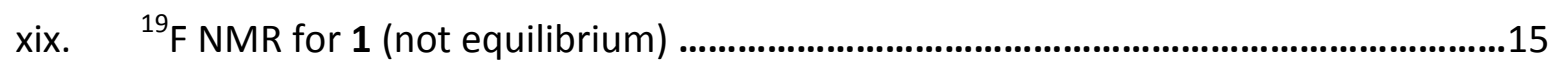

xx. 1D slice of $\mathrm{H}-2$ from $2 \mathrm{D}^{1} \mathrm{H}-{ }^{1} \mathrm{H}$ NOESY NMR spectrum of $\mathbf{1}$ ( $\alpha$-pyranose).................. 15

xxi. 1D slice of $\mathrm{H}-16(\mathrm{H}-2)$ from $2 \mathrm{D}^{1} \mathrm{H}^{-1} \mathrm{H}$ NOESY NMR spectrum of $\mathbf{1}$ ( $\beta$-pyranose)........16

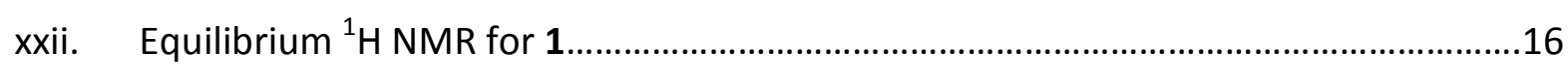

xxiii. Comparison Table for ${ }^{1} \mathrm{H}$ NMR Data Supplied by Toyokuni et al. and Caille et al.....17

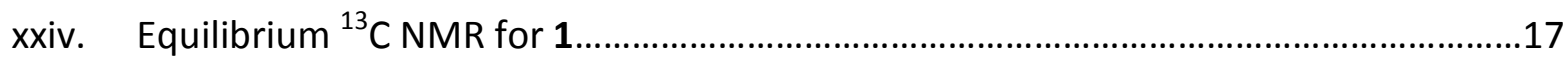

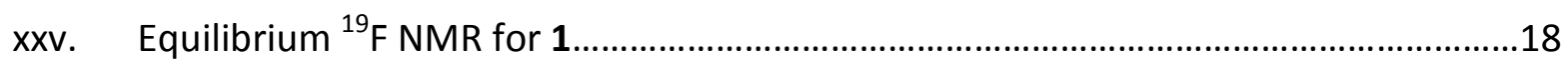

xxvi. M06-2X/6-31+G(d,p) Gibbs Free Energies, Boltzmann Populations, Total Energies, and Zero-point Energy Corrections for Conformational Ensembles of Systems A-D Within Free Energy Window of $4 \mathrm{kcal} / \mathrm{mol}$; Gas Phase. ("6a" = species A; "6b" =

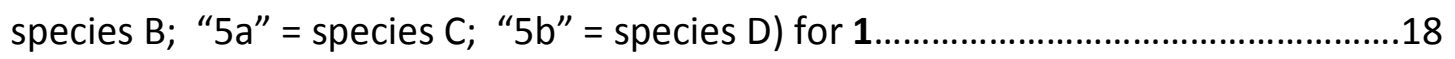


i. ${ }^{1} \mathrm{H} \quad \mathrm{NMR}$ for $(2 S, 3 S, 4 S, 5 S)$-benzyl 3,4-dihydroxy-5-methoxytetrahydrofuran-2carboxylate (5a):

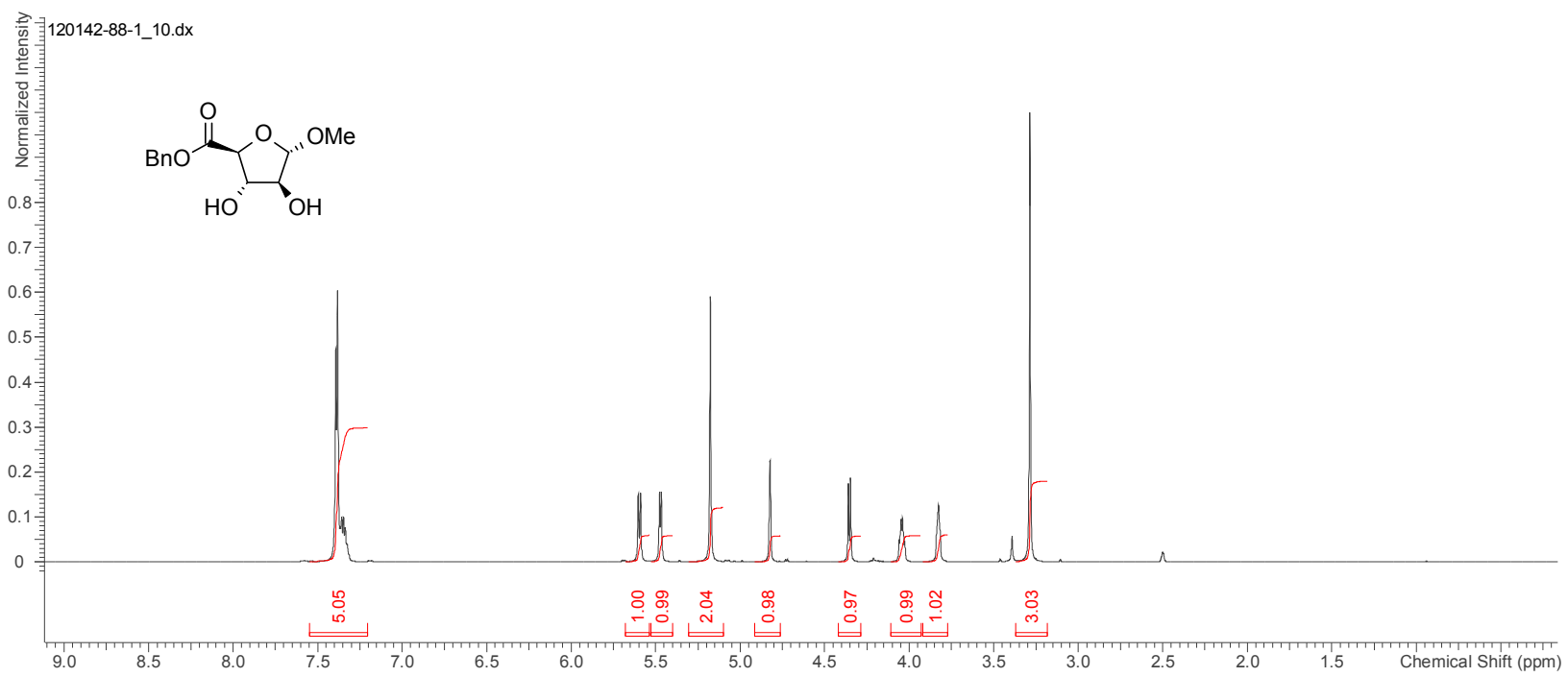

ii. $\quad H-1 \quad{ }^{1} \mathrm{H} \quad$ NMR expansions for $(2 R / S, 3 S, 4 S, 5 S)$-benzyl 3,4-dihydroxy-5methoxytetrahydrofuran-2-carboxylate $(5 a / 5 b)$ :

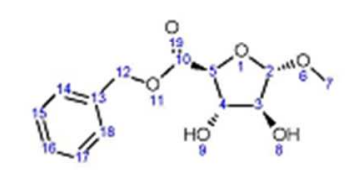

benzyl methyl a.D-arabinoturanosiduronate

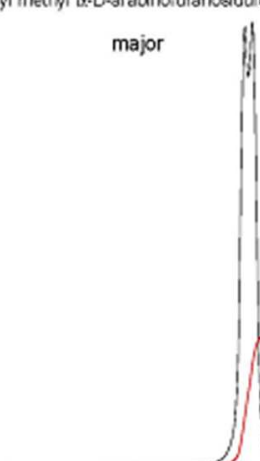

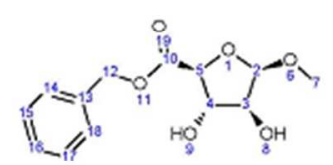

benzyl metryl $\beta$-D-arabinoturanosiduronate

minor

major

2
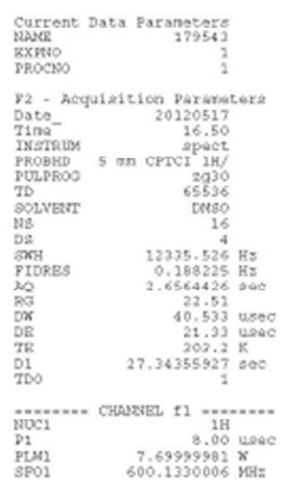

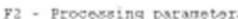

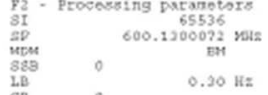

2 
iii. ${ }^{13} \mathrm{C}$ NMR for $(2 S, 3 S, 4 S, 5 S)$-benzyl 3,4-dihydroxy-5-methoxytetrahydrofuran-2carboxylate (5a):

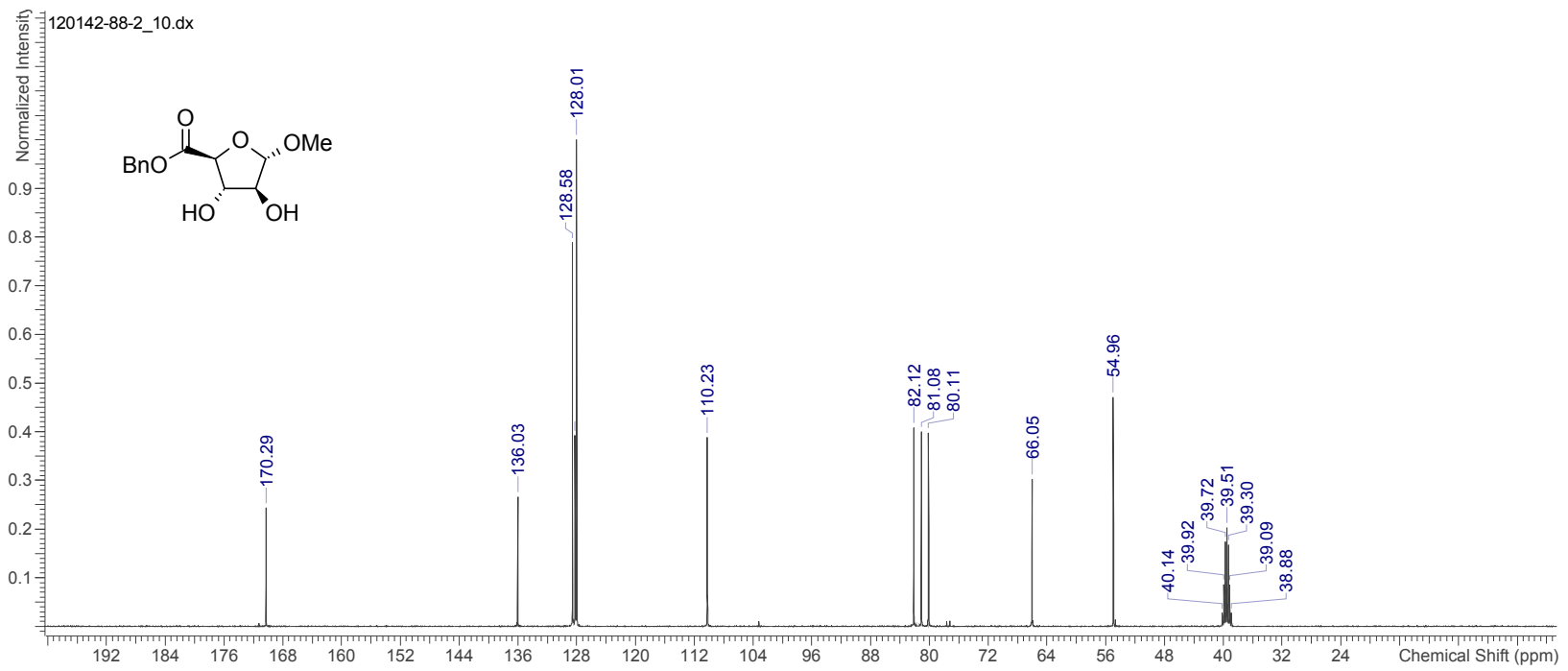


iv. Representative LCMS Chromatograms of $5 \mathrm{a}$ and $5 \mathrm{~b}$ :

Note: LCMS ratios of $\mathbf{5 a}$ and $\mathbf{5} \mathbf{b}$ were corroborated by ${ }^{1} \mathrm{H}$ NMR ratios in multiple instances.

First chromatogram (99/1): $\mathbf{5 a}$ at 3.72 minand $\mathbf{5 b}$ at $3.94 \mathrm{~min}$.
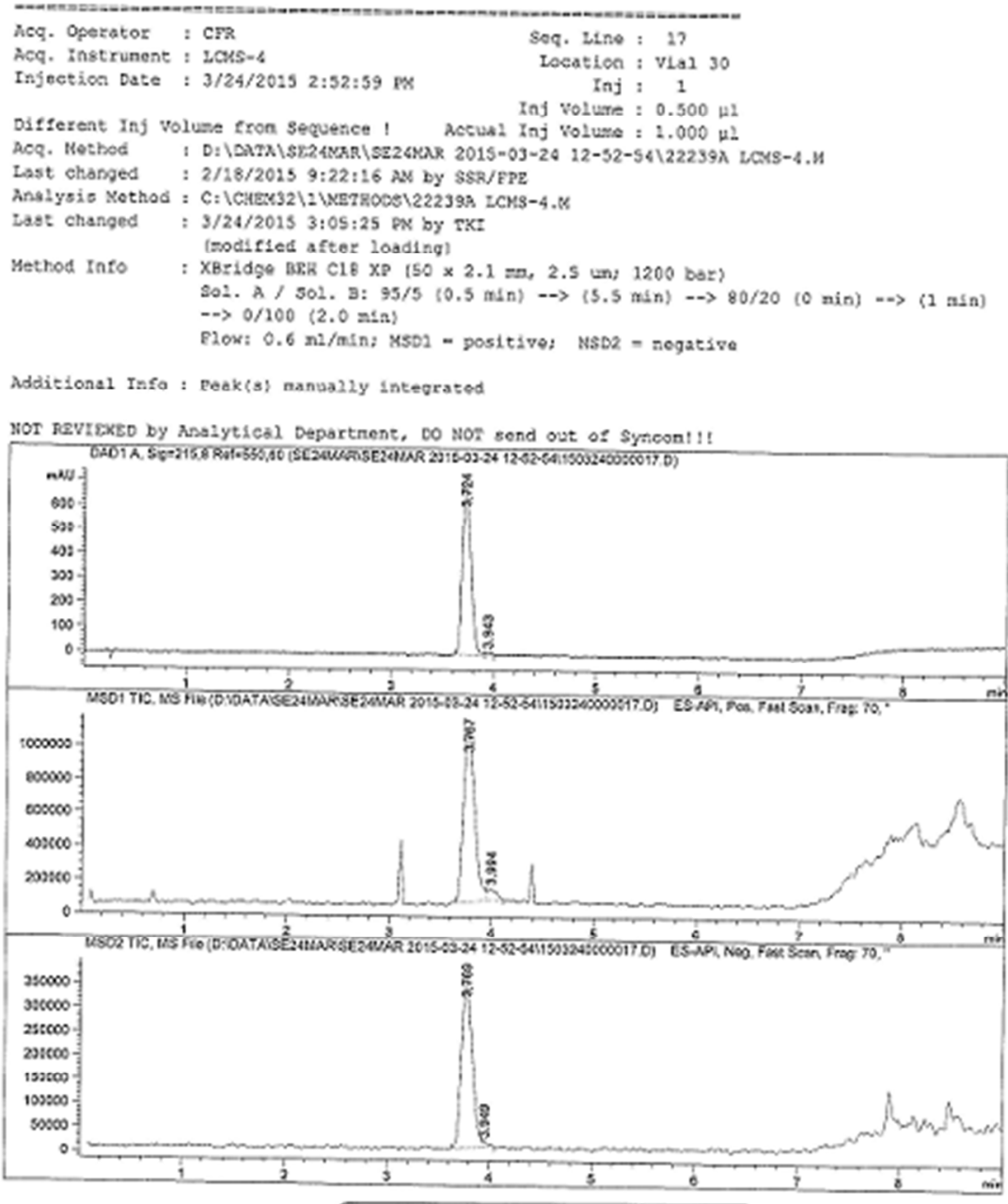

$\begin{array}{lll}\text { Sorted By } & : & \text { Signal } \\ \text { Nultiplier } & : & 1.0000 \\ \text { Dilution } & : & 1.0000\end{array}$

Do not use Multipliex \& Dilution Factor with ISrDs

Signal 1: DADl A, Sig-215, \& Ref-550,60

\begin{tabular}{|c|c|c|c|c|c|c|}
\hline $\begin{array}{c}\text { Peat } x \\
k\end{array}$ & $\begin{array}{c}\text { RetTime } \\
\text { [min] }\end{array}$ & Type & $\begin{array}{l}\text { Width } \\
\text { [min] }\end{array}$ & $\begin{array}{c}\text { Arca } \\
(m \mathrm{AU} * \mathrm{~s}]\end{array}$ & $\begin{array}{l}\text { Hesght } \\
\text { [mAd] }\end{array}$ & $\begin{array}{c}\text { Area } \\
8\end{array}$ \\
\hline \multicolumn{7}{|c|}{ 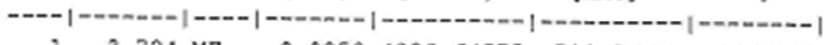 } \\
\hline 1 & 3.324 & uF & 0.0950 & 4039.64535 & 708.75049 & 99.0053 \\
\hline 2 & 3.943 & EN & 0.0590 & 40.58522 & 12.46483 & 0.9947 \\
\hline Totel & $s:$ & & & 4080.23098 & 220.22532 & \\
\hline
\end{tabular}




\section{Second chromatogram (95.7/4.3): 5a at 3.81 minand 5 b at 4.07 min.}
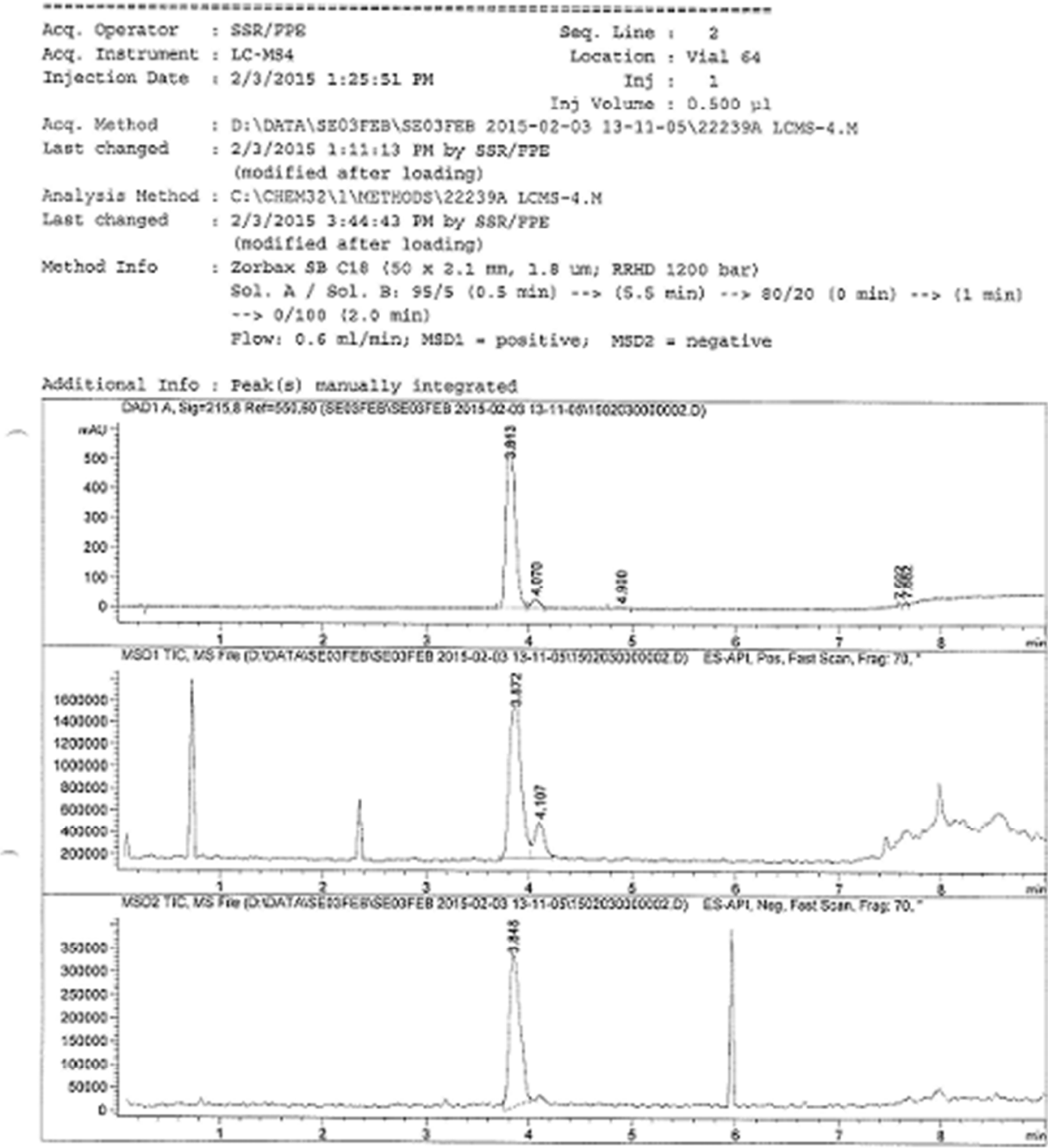

$\begin{array}{lll}\text { Sorted By } & : & \text { Signal } \\ \text { wultiplier } & : & 1.0000 \\ \text { Dilution } & : & 1.0000\end{array}$

Do not use Multiplier \& Dilution Factor with ISTDs

Signal 1: DAD1 A, Sig-215,8 Ref-550,60

\begin{tabular}{|c|c|c|c|c|c|c|}
\hline $\begin{array}{c}\text { Peak } \\
\#\end{array}$ & $\begin{array}{c}\text { RetTime } \\
\text { [min] }\end{array}$ & Type & $\begin{array}{l}\text { Wiath } \\
\text { [min] }\end{array}$ & $\begin{array}{c}\text { Area } \\
{[\text { mAU*s] }}\end{array}$ & $\begin{array}{l}\text { Height } \\
\text { [mAv] }\end{array}$ & $\begin{array}{c}\text { Area } \\
\div\end{array}$ \\
\hline & & & $\ldots$ & | & - & - \\
\hline 2 & 3.813 & MF & 0.1004 & 3544.68433 & 588.54803 & 94.8071 \\
\hline 2 & 4.070 & $F$ & 0.0963 & 156.52051 & 27.07830 & 4.1863 \\
\hline 3 & 4.900 & $\mathrm{NS}$ & 0.0972 & 20.04137 & 3.43781 & 0.5360 \\
\hline 4 & 7.592 & $\mathrm{MM}$ & 0.0220 & 11.40931 & 8.62674 & 0.3052 \\
\hline 5 & 7.662 & $\mathrm{NOM}$ & 0.0137 & 6.28124 & 3.52438 & 0.1653 \\
\hline & & & & 3738 & 2526 & \\
\hline
\end{tabular}



v. ${ }^{1} \mathrm{H}$
NMR
for
$(2 S, 3 R, 4 S, 5 S)$-benzyl
5-methoxy-3,4-
bis((trimethylsilyl)oxy)tetrahydrofuran-2-carboxylate (6):

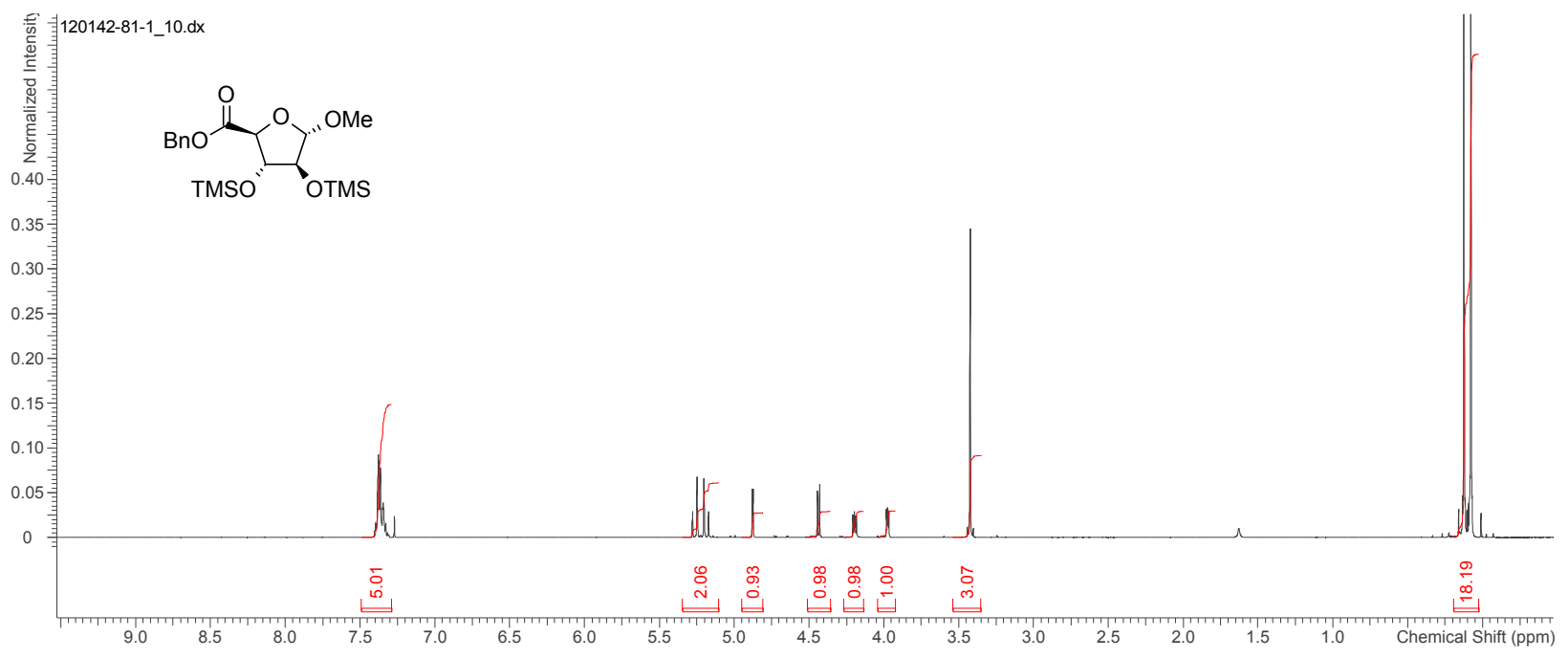
vi. $\quad{ }^{13} \mathrm{C}$
NMR for
$(2 S, 3 R, 4 S, 5 S)$-benzyl
5-methoxy-3,4-
bis((trimethylsilyl)oxy)tetrahydrofuran-2-carboxylate (6):

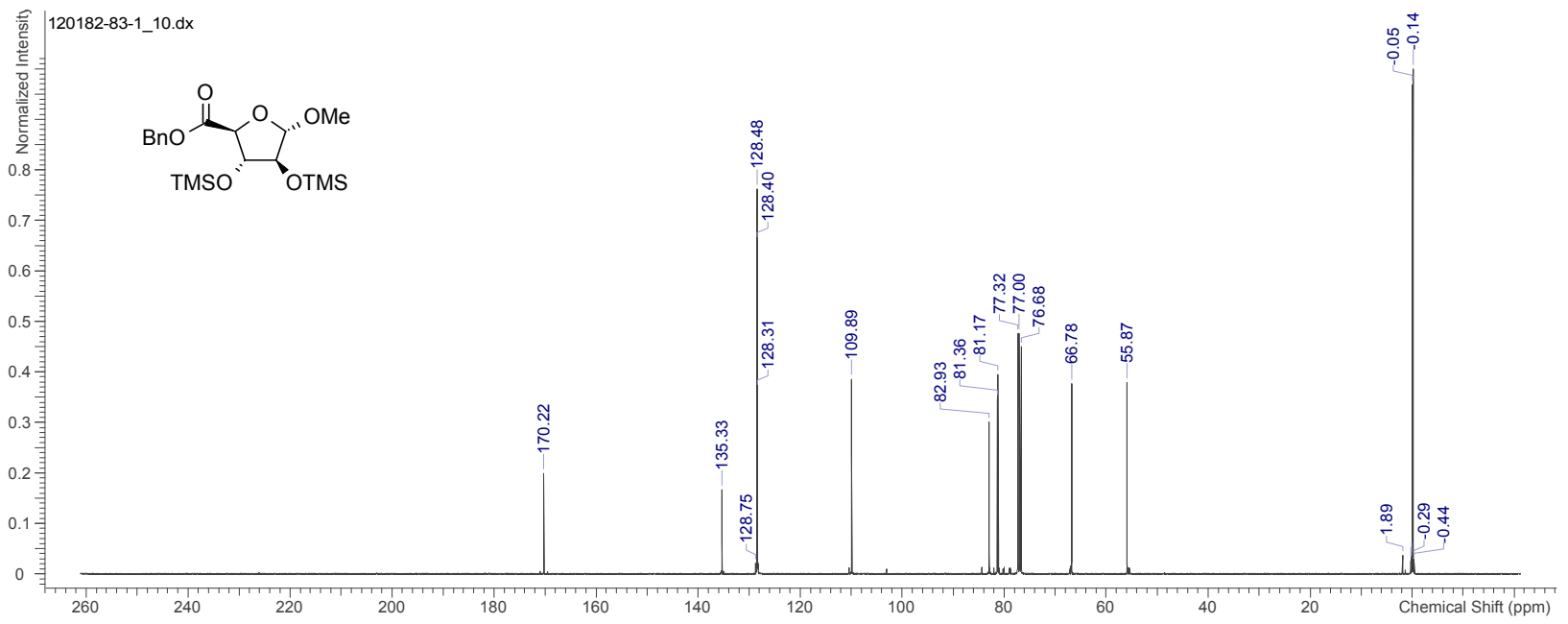


vii. ${ }^{1} \mathrm{H} \quad \mathrm{NMR}$ for $\quad(((2 S, 3 R, 4 S, 5 S)-2-(1-$ (benzyloxy)-2,2,2-trifluoro-1((trimethylsilyl)oxy)ethyl)-5-methoxytetrahydrofuran-3,4-

diyl)bis(oxy))bis(trimethylsilane) (7):

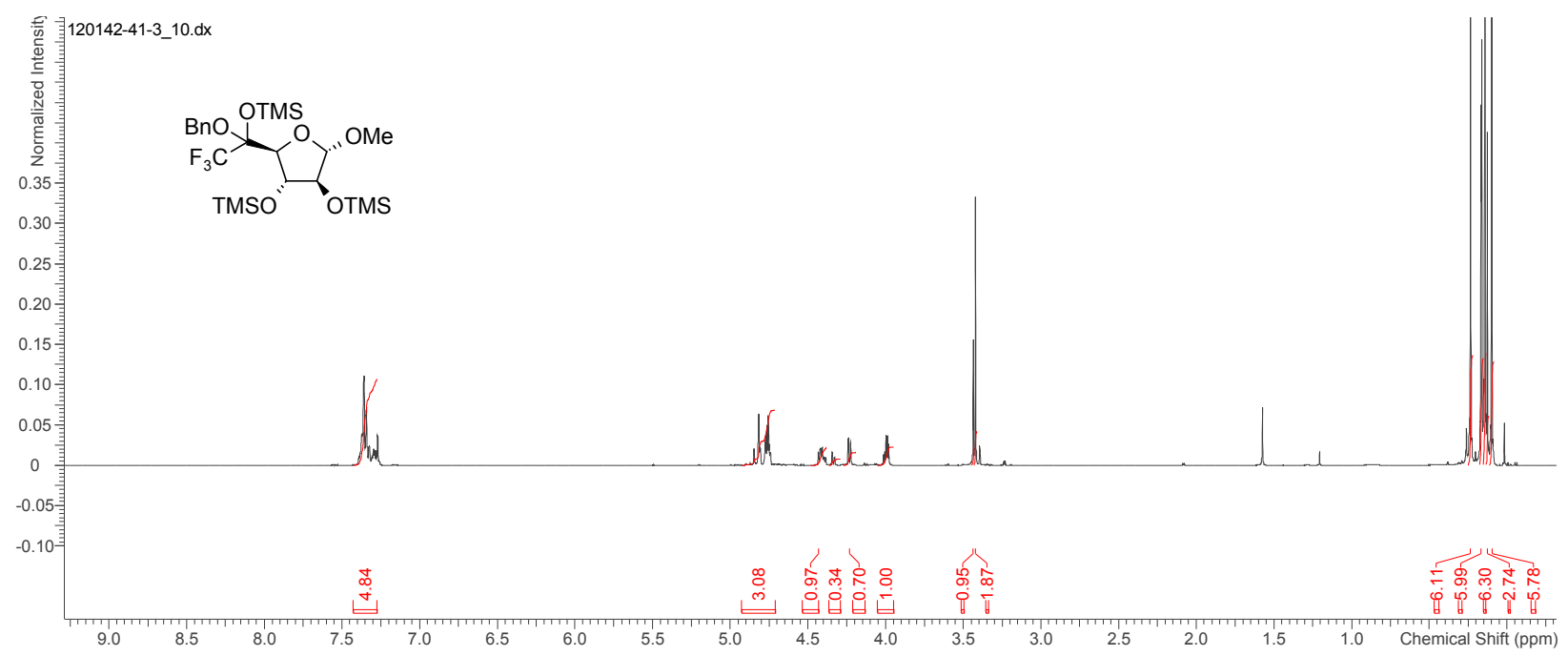

viii. ${ }^{13} \mathrm{C} \quad \mathrm{NMR}$ for $\quad(((2 S, 3 R, 4 S, 5 S)-2$-(1-(benzyloxy)-2,2,2-trifluoro-1((trimethylsilyl)oxy)ethyl)-5-methoxytetrahydrofuran-3,4-

diyl)bis(oxy))bis(trimethylsilane) (7):

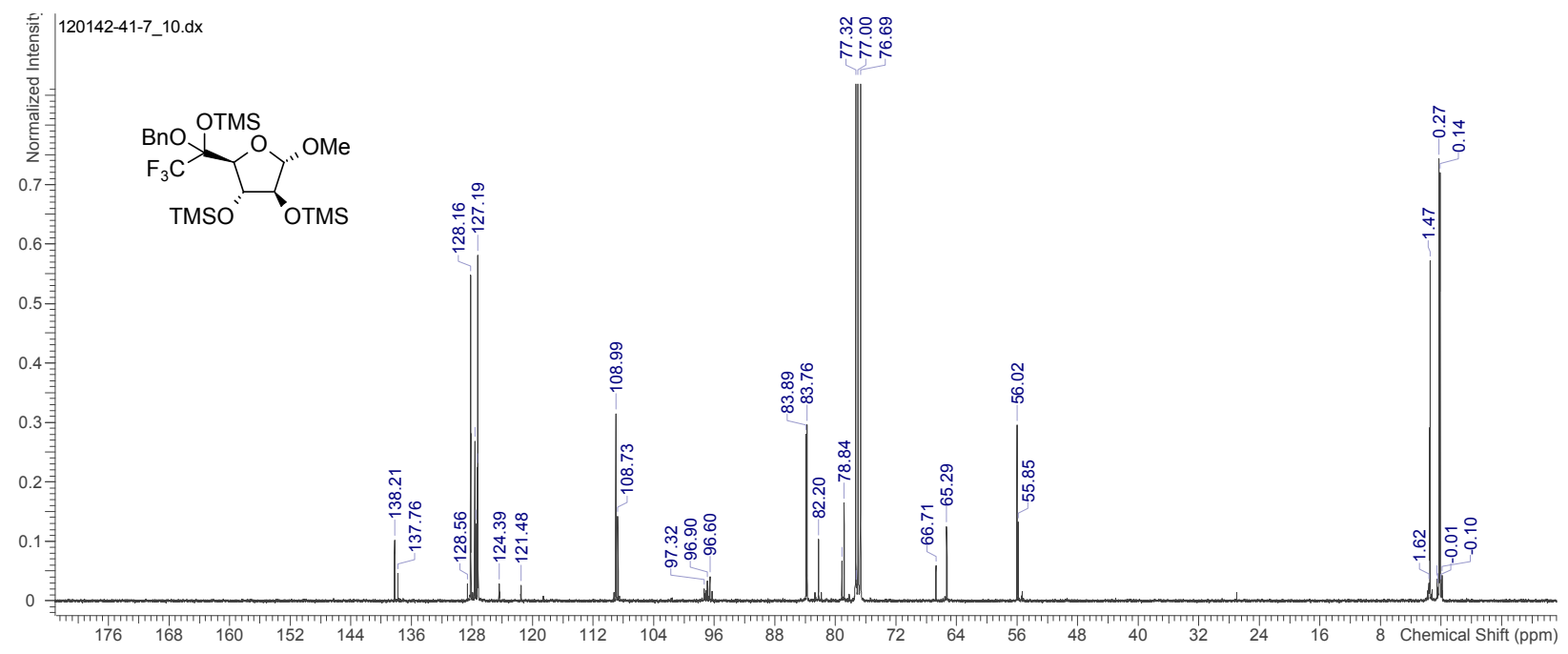


ix. ${ }^{19} \mathrm{~F} \quad \mathrm{NMR}$ for $\quad(((2 S, 3 R, 4 S, 5 S)-2$-(1-(benzyloxy)-2,2,2-trifluoro-1-

((trimethylsilyl)oxy)ethyl)-5-methoxytetrahydrofuran-3,4-

diyl)bis(oxy))bis(trimethylsilane) (7):

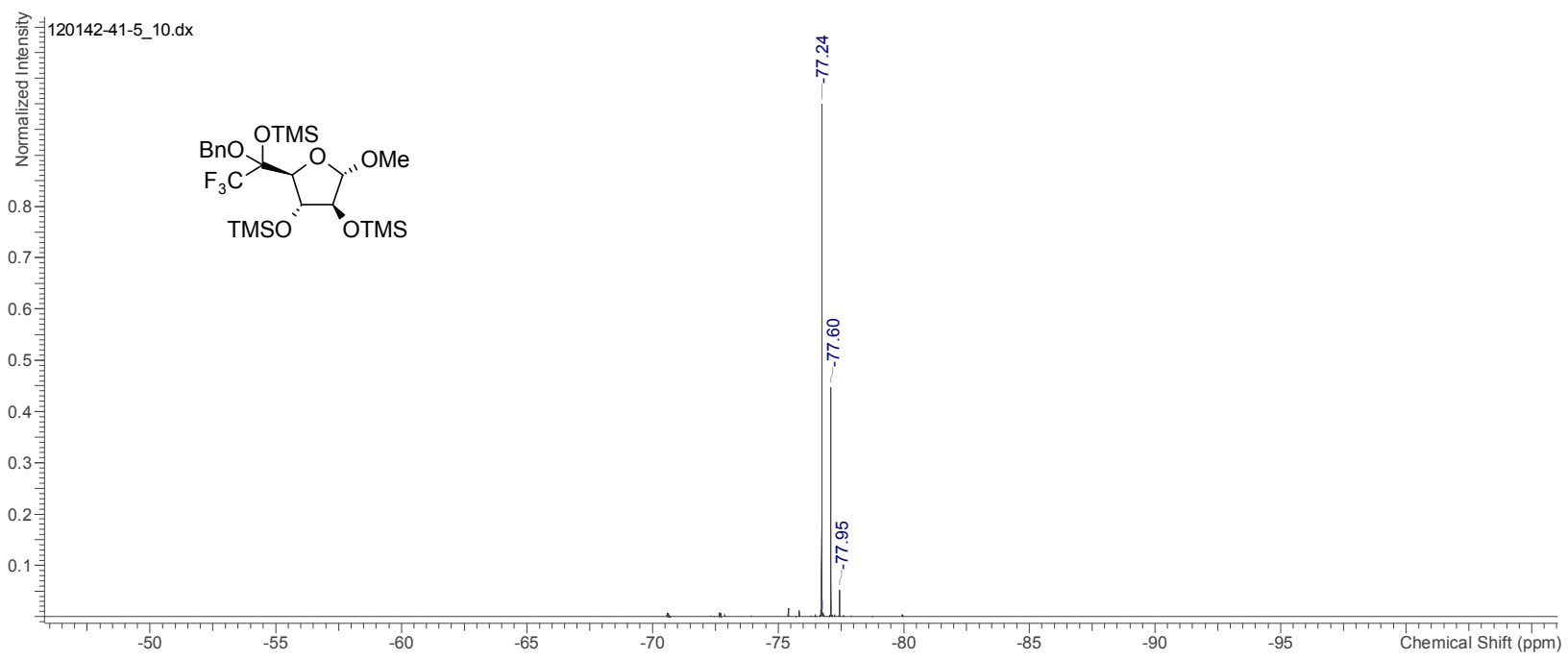

x. $\quad{ }^{1}$ H NMR for 11:

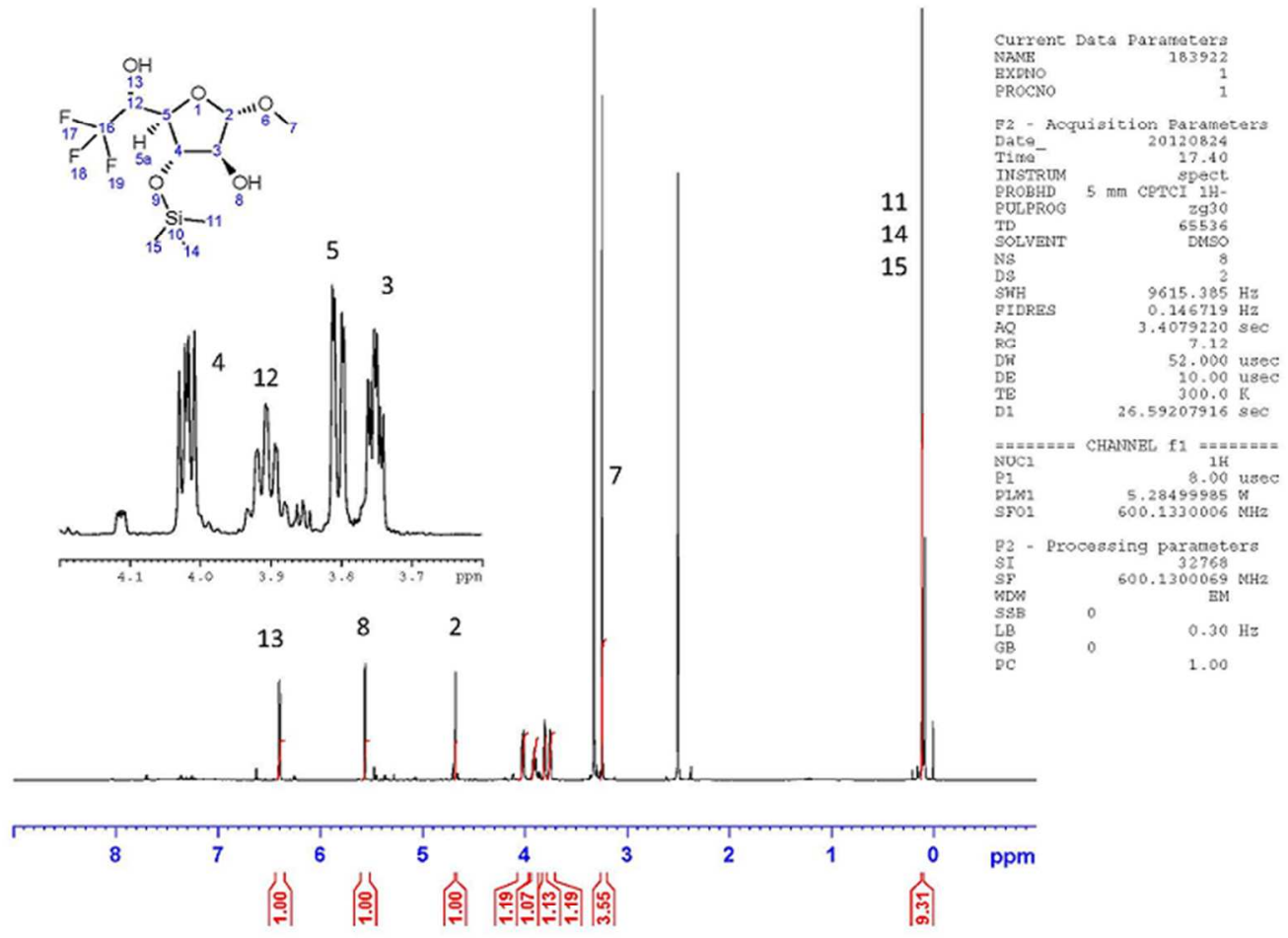

Figure 3. 1D ${ }^{1} \mathrm{H}$ NMR spectrum of TMS-intermediate, lot 116961-75-1 in DMSO-d ${ }_{6}$. 
xi. $\quad 2 \mathrm{D}{ }^{1} \mathrm{H}-{ }^{1} \mathrm{H}$ COSY NMR spectrum for 11 :
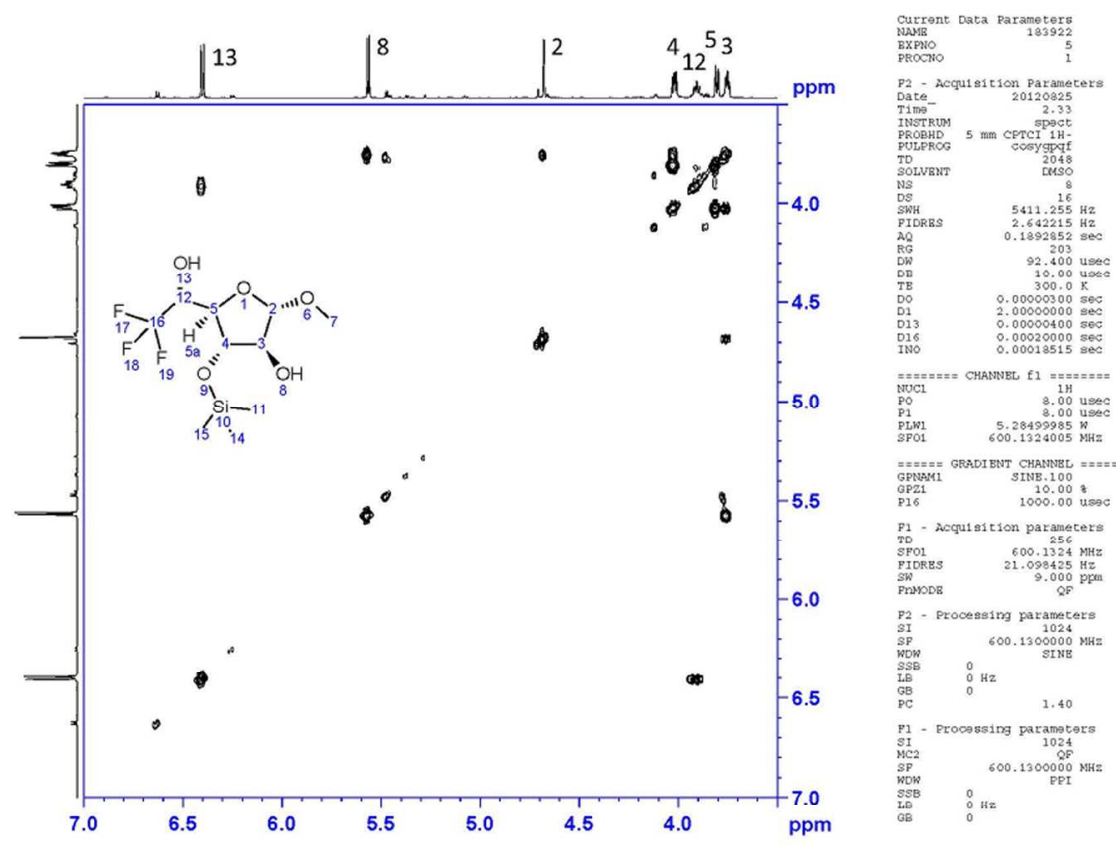

Figure 4. $2 \mathrm{D}^{1} \mathrm{H}-{ }^{1} \mathrm{H}$ COSY NMR spectrum of TMS-intermediate, lot 116961-75-1 in DMSO-d .

xii. $\quad{ }^{13}$ C NMR for 11:
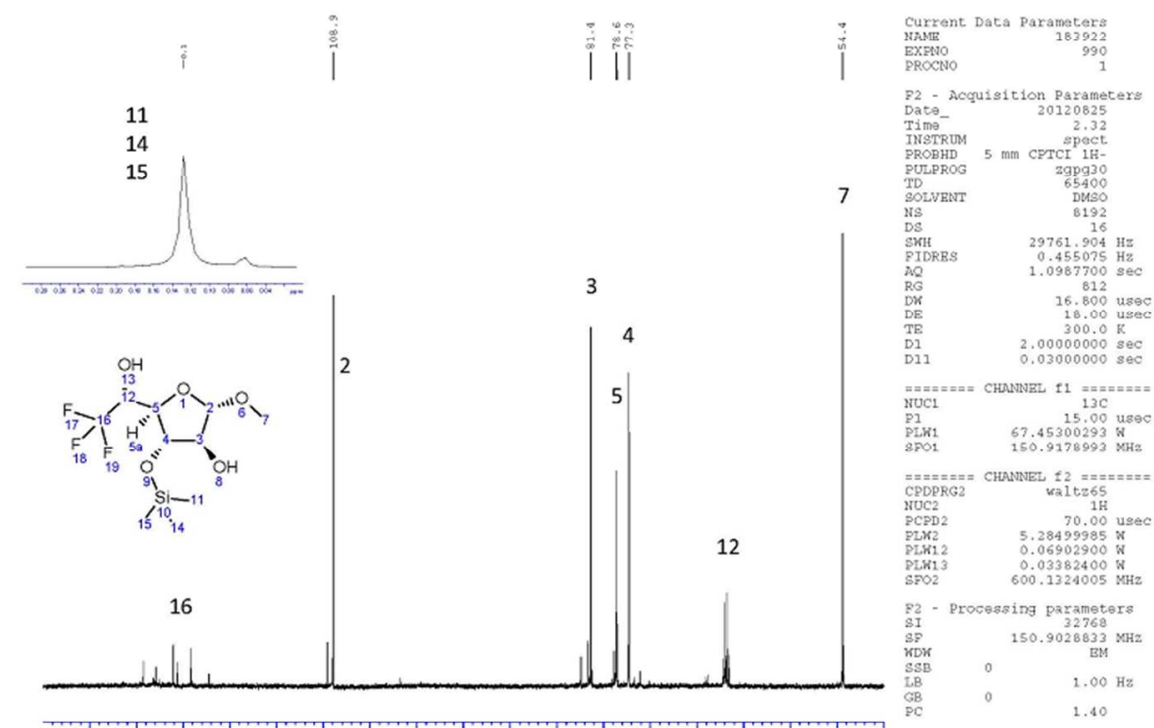

$\begin{array}{lllllllllllllllll}135 & 130 & 125 & 120 & 115 & 110 & 105 & 100 & 95 & 90 & 85 & 80 & 75 & 70 & 65 & 60 & \mathrm{ppm}\end{array}$

Figure 6. $1 \mathrm{D}^{13} \mathrm{C}$ NMR spectrum of TMS-intermediate, lot 116961-75-1 in DMSO- $\mathrm{d}_{6}$. 
xiii. ${ }^{1} \mathrm{H} \quad \mathrm{NMR}$ for $\quad(2 S, 3 S, 4 S, 5 S)-2$-methoxy-5-((R)-2,2,2-trifluoro-1hydroxyethyl)tetrahydrofuran-3,4-diol (12 major):

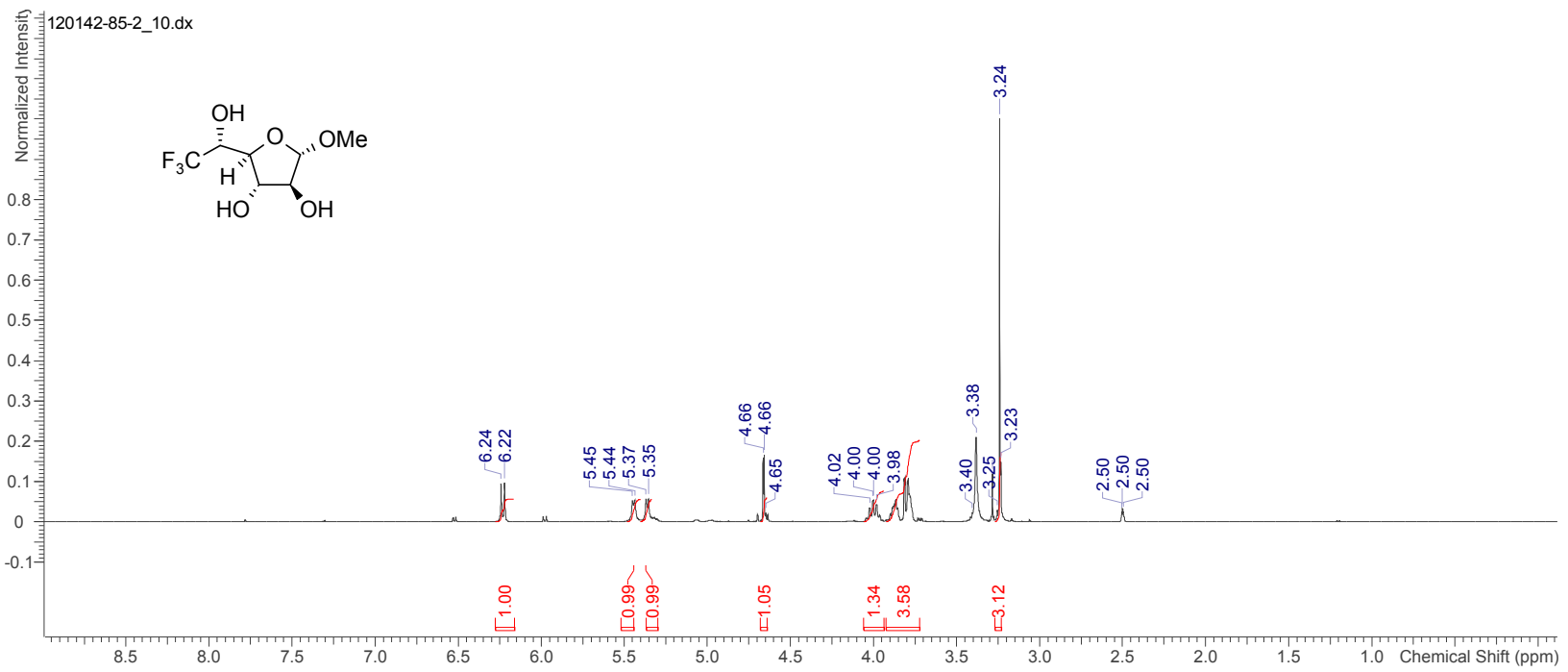

xiv. ${ }^{13} \mathrm{C}$ NMR for $\quad(2 S, 3 S, 4 S, 5 S)-2$-methoxy-5-((R)-2,2,2-trifluoro-1hydroxyethyl)tetrahydrofuran-3,4-diol (12 major):

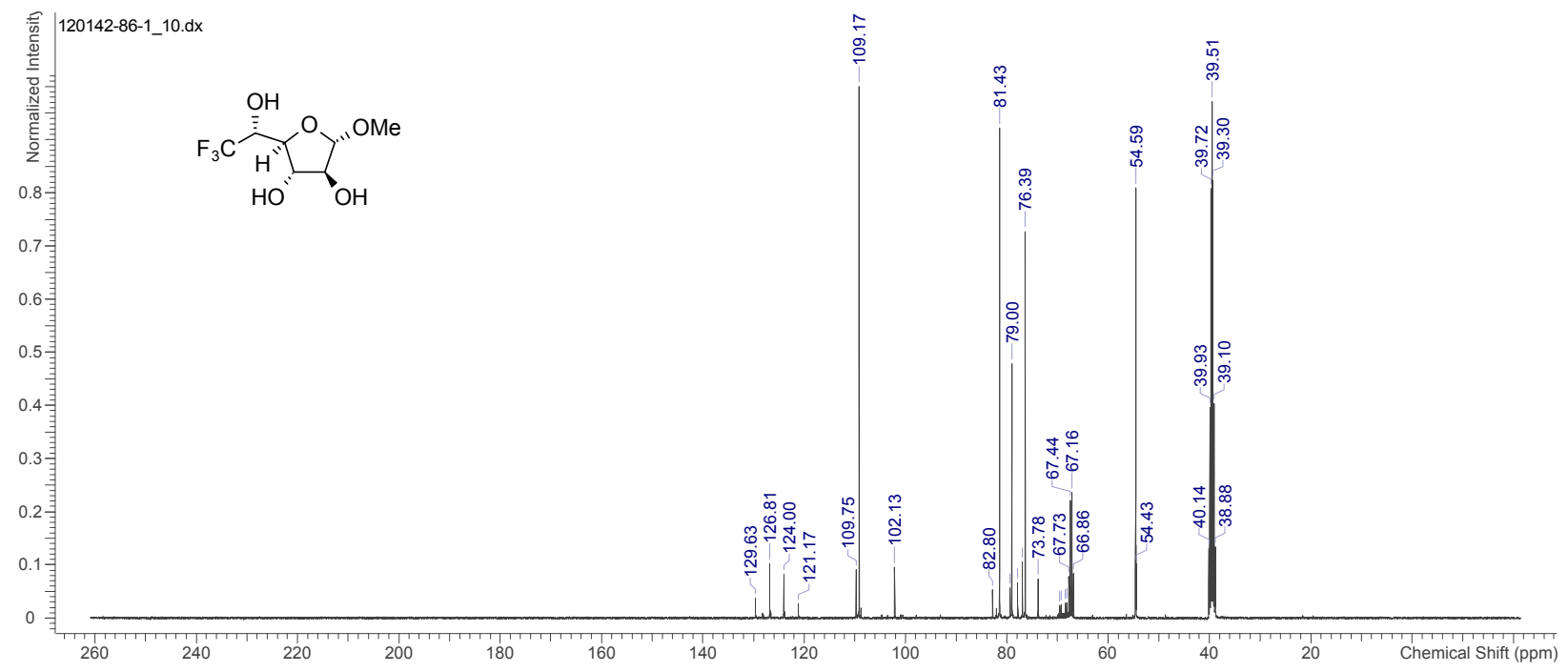


xV. ${ }^{19} \mathrm{~F} \quad \mathrm{NMR}$ for $\quad(2 S, 3 S, 4 S, 5 S)-2$-methoxy-5-((R)-2,2,2-trifluoro-1hydroxyethyl)tetrahydrofuran-3,4-diol (12 major):

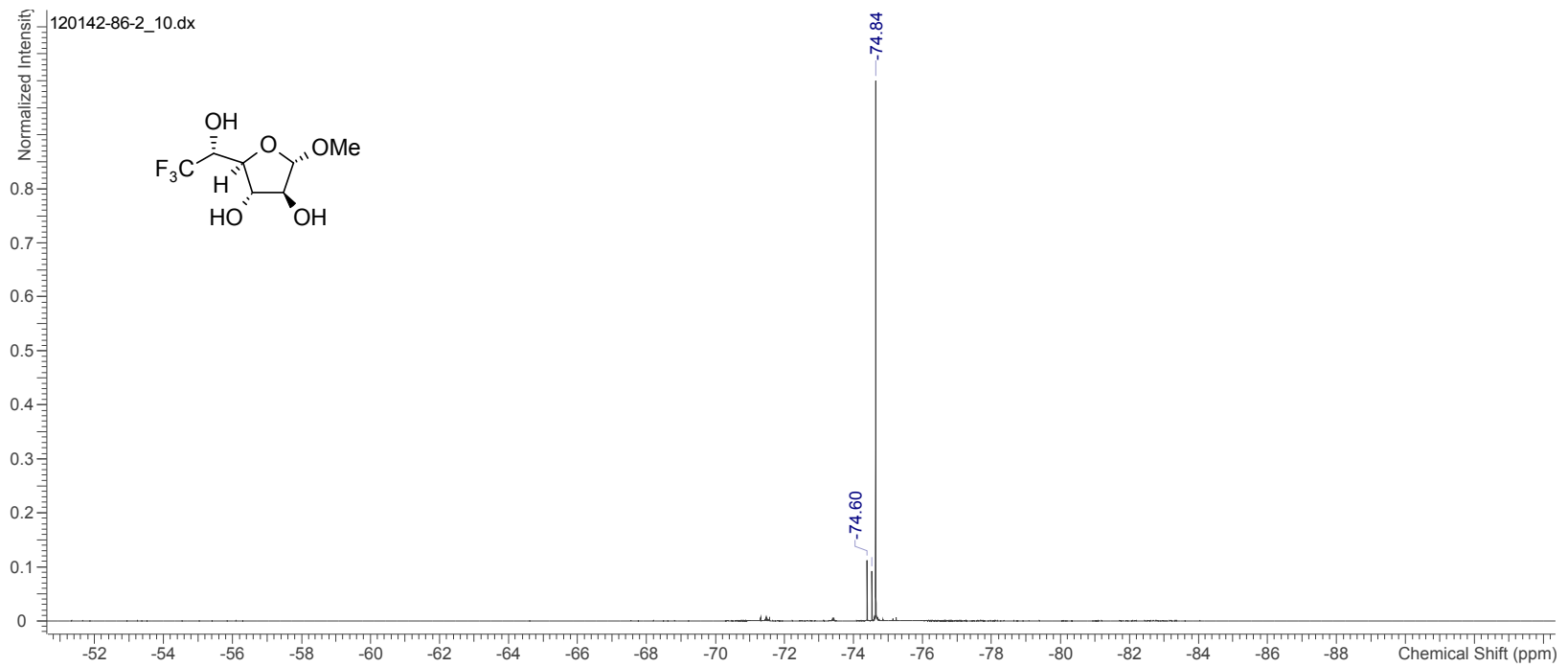


xvi. Representative GC Chromatogram of $\mathbf{1 2}$ and 13:

Note: GC ratios of 12 and 13 were corroborated by ${ }^{1} \mathrm{H}$ NMR and ${ }^{19} \mathrm{~F}$ NMR ratios.

Desired Triol is $\mathbf{1 2}$ at $6.98 \mathrm{~min}$ and undesired triol is $\mathbf{1 3}$ at $\mathbf{7 . 3 5} \mathrm{min}$.

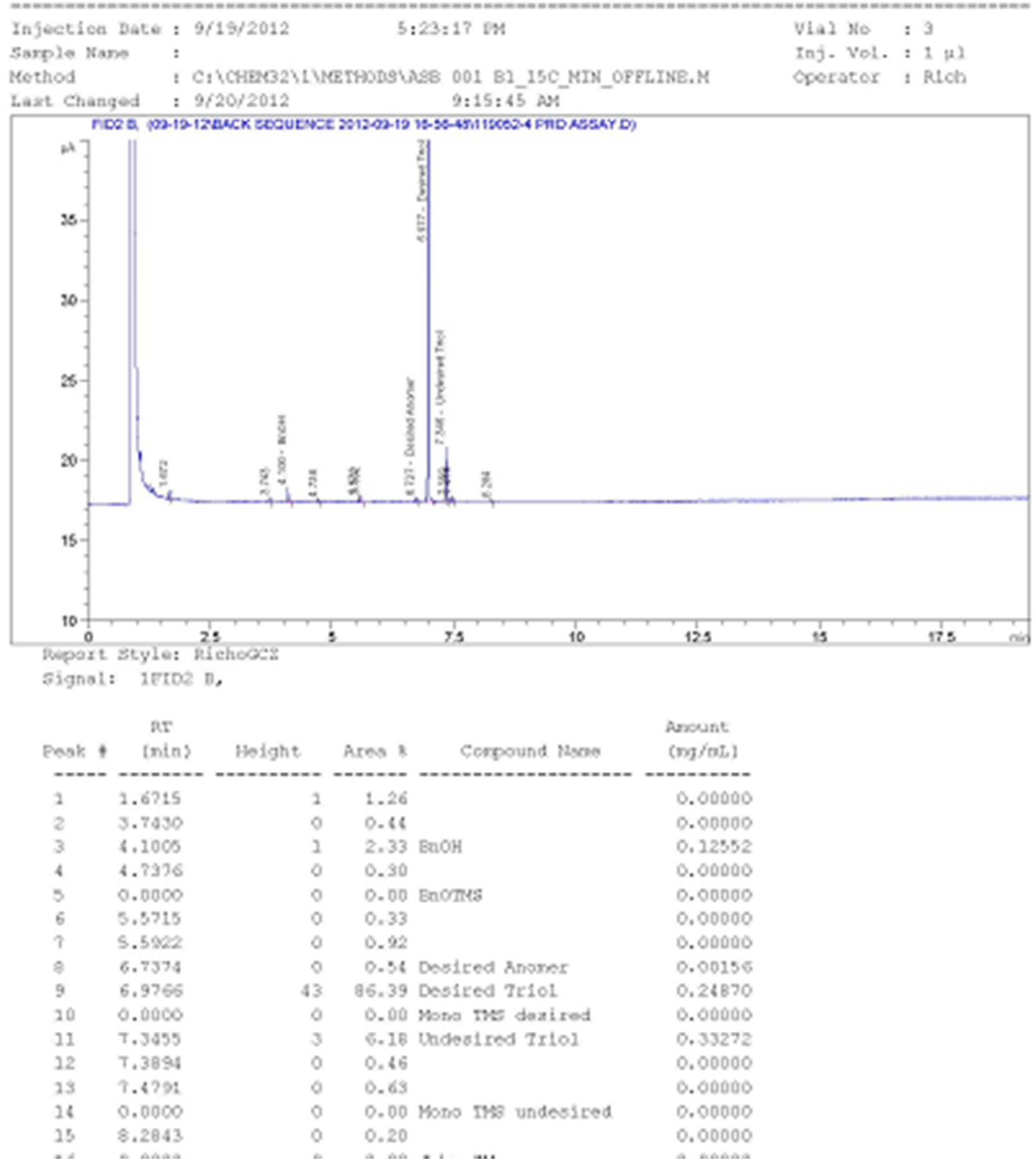


xvii. ${ }^{1}$ H NMR for 1 (not equilibrium):
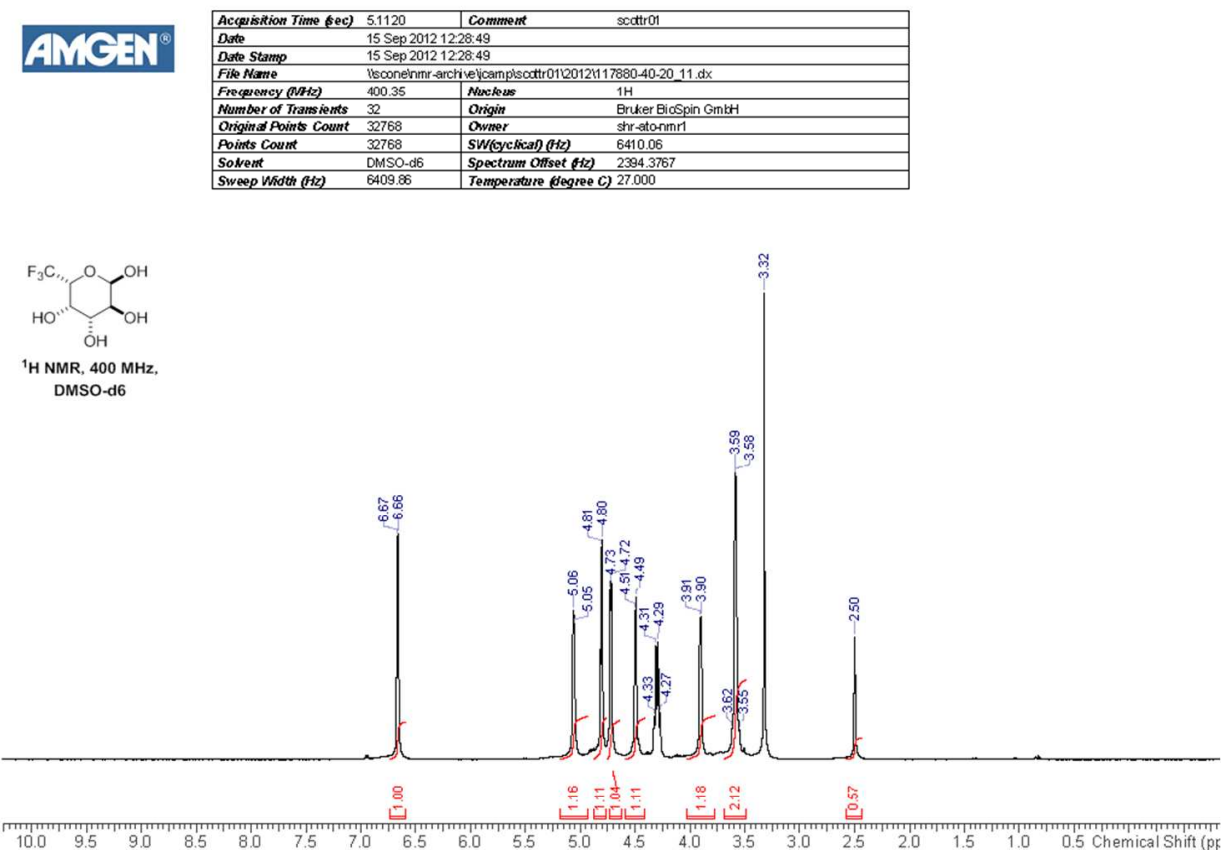

xviii. $\quad{ }^{13} \mathrm{C}$ NMR for $\mathbf{1}$ (not equilibirum):
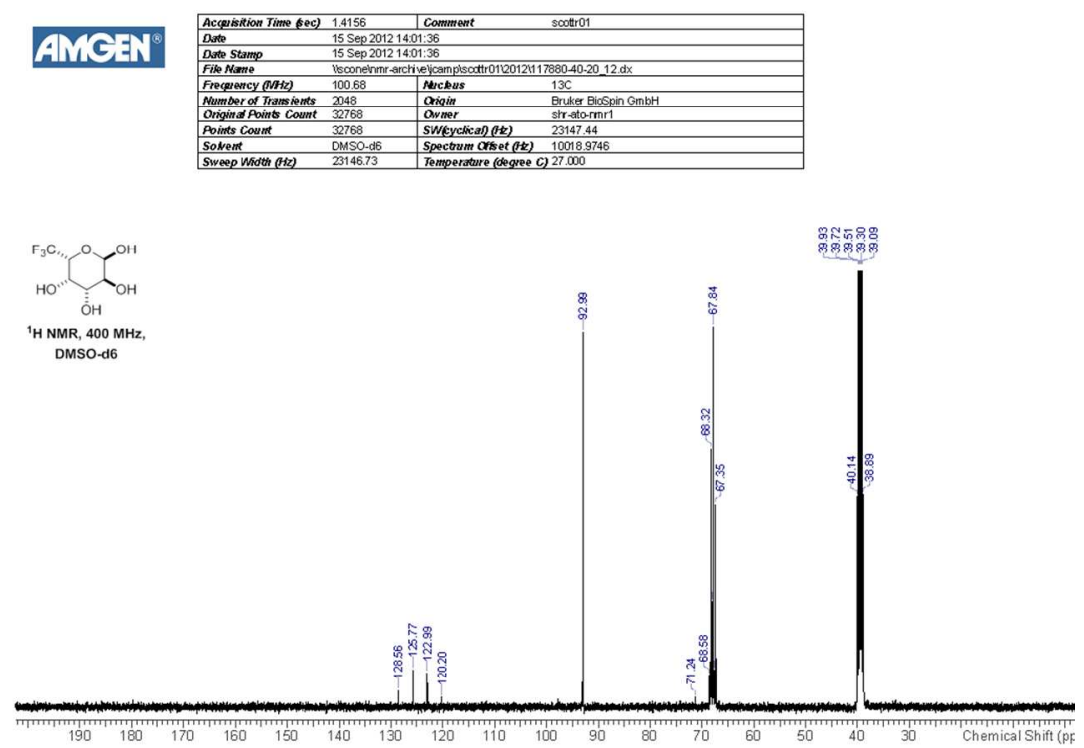
xix. $\quad{ }^{19}$ F NMR for $\mathbf{1}$ (not equilibrium):

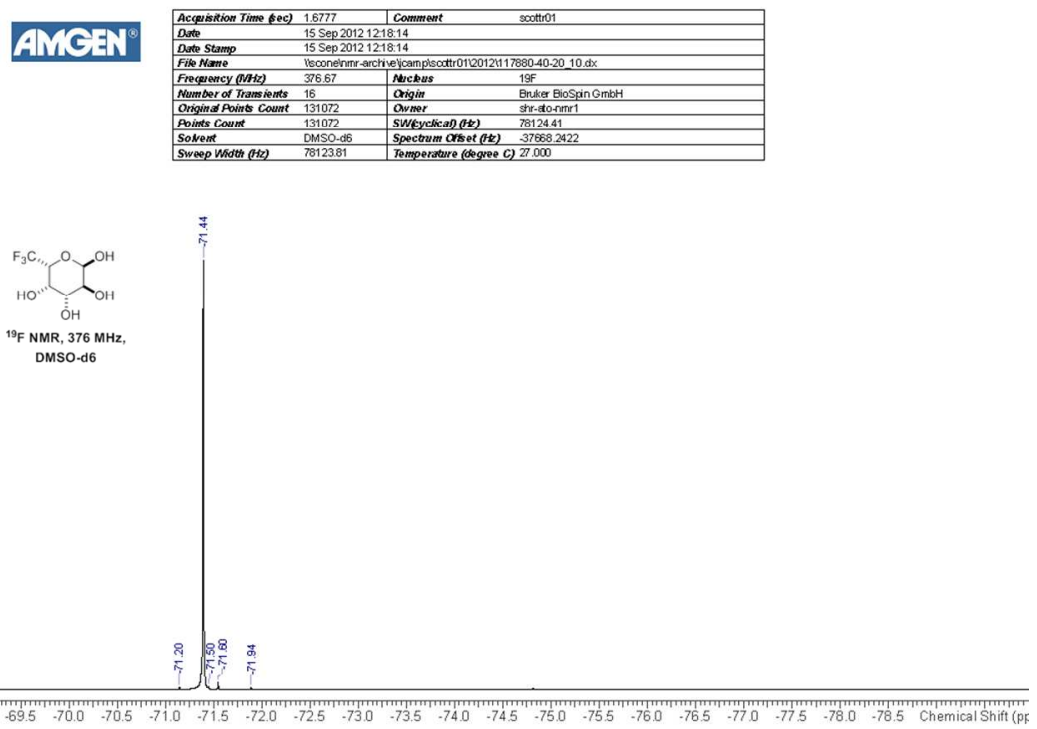

xx. $\quad$ 1D slice of $\mathrm{H}-2$ from $2 \mathrm{D}{ }^{1} \mathrm{H}-{ }^{1} \mathrm{H}$ NOESY NMR spectrum of $\mathbf{1}$ ( $\alpha$-pyranose):

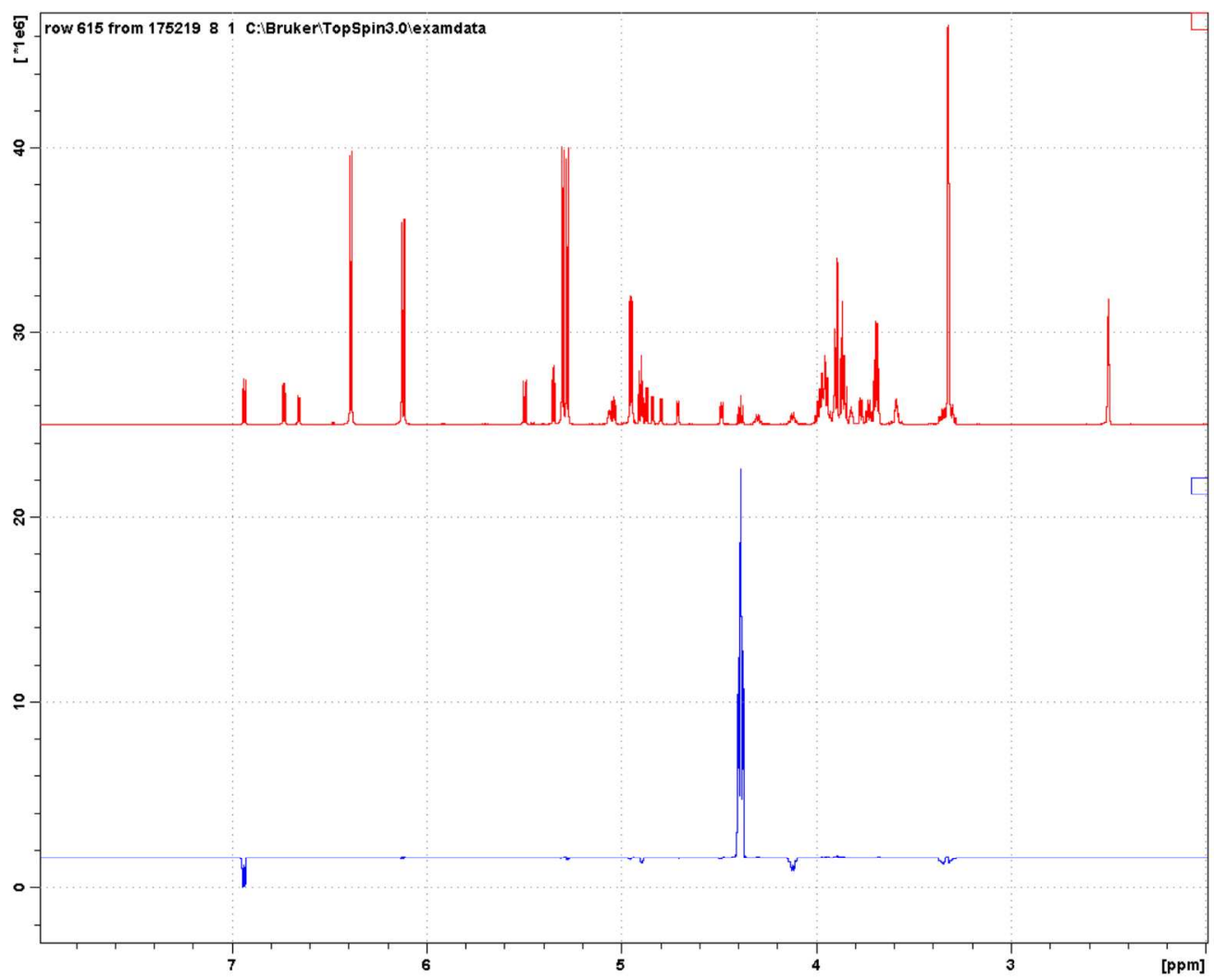


xxi. 1D slice of $\mathrm{H}-16(\mathrm{H}-2)$ from $2 \mathrm{D}^{1} \mathrm{H}-{ }^{1} \mathrm{H}$ NOESY NMR spectrum of $\mathbf{1}$ ( $\beta$-pyranose):

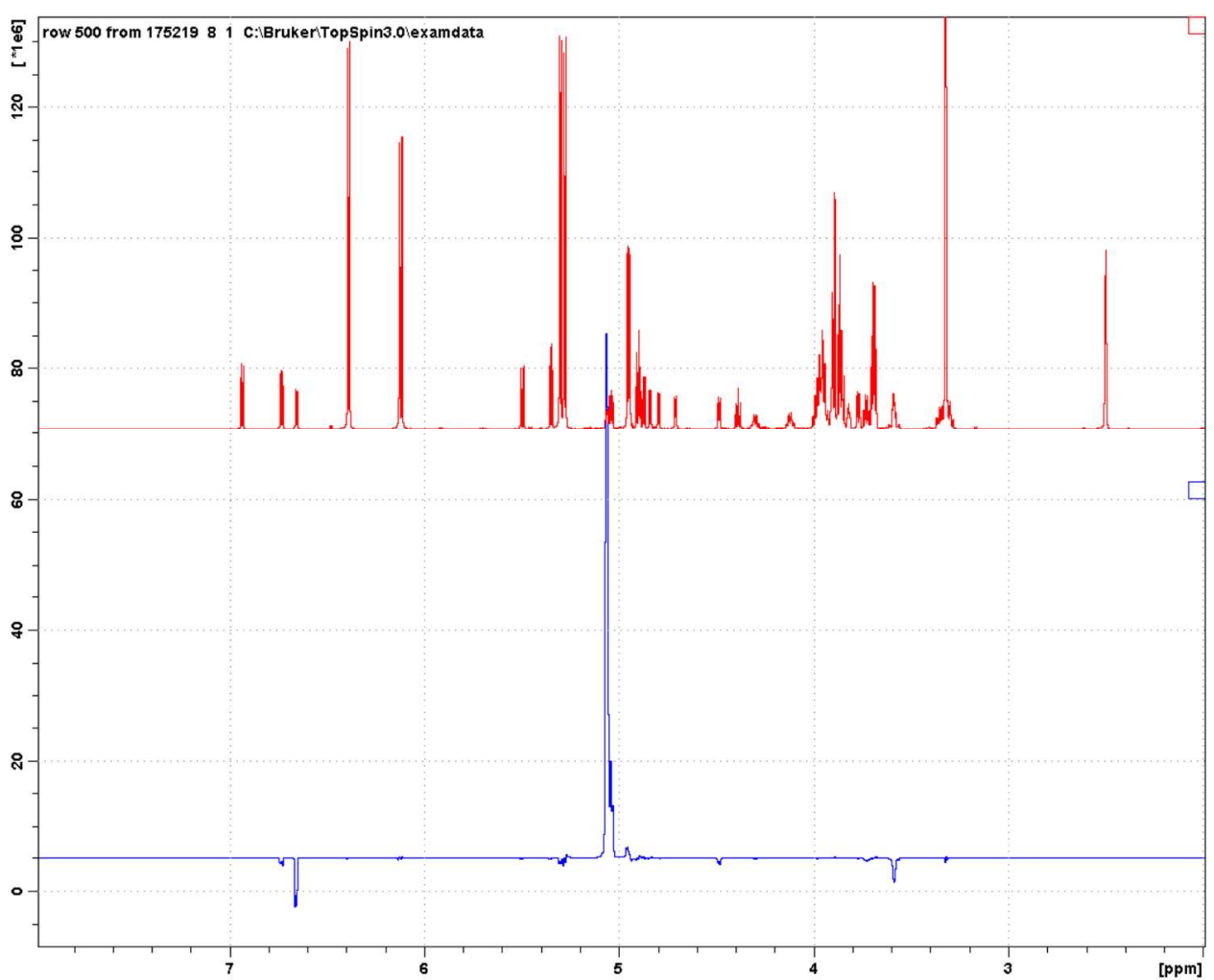

xxii. Equilibrium ${ }^{1} \mathrm{H}$ NMR for $\mathbf{1}$ :
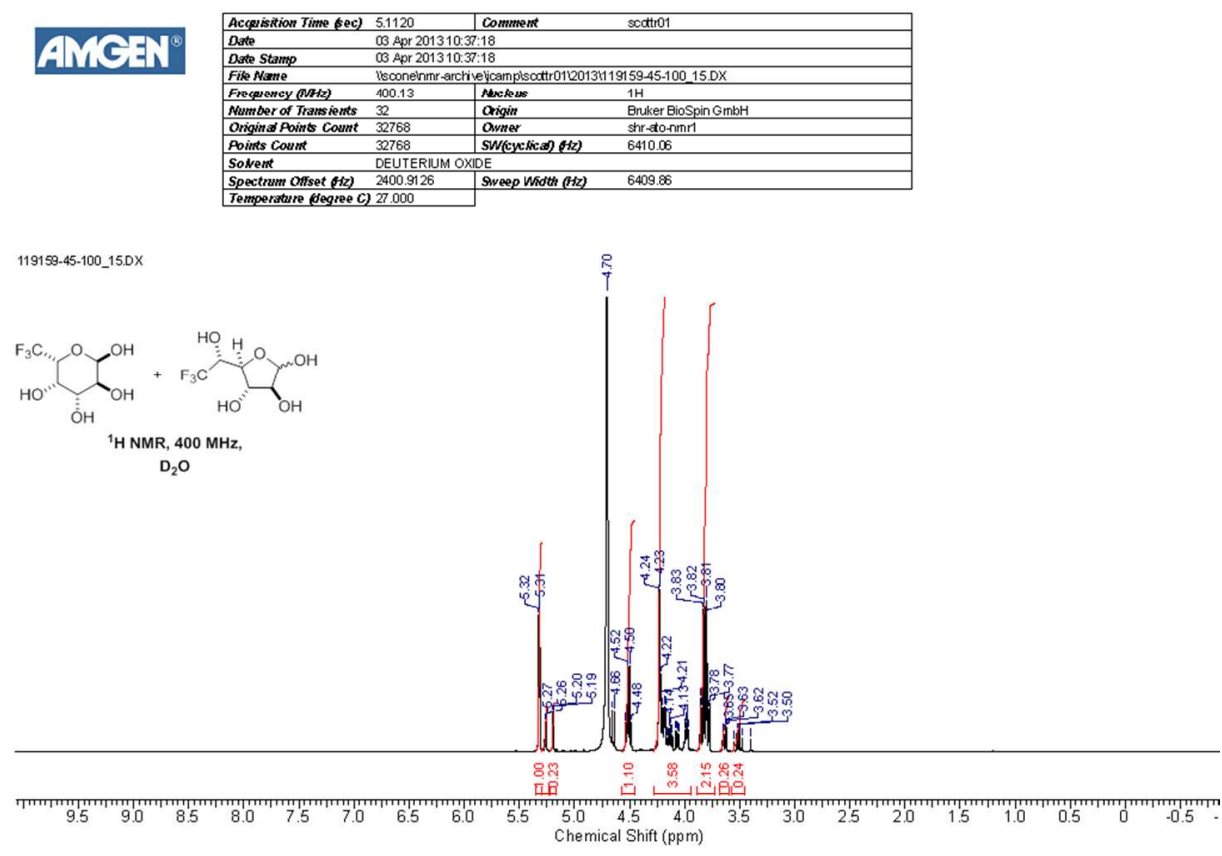
xxiii. Comparison Table for ${ }^{1} \mathrm{H}$ NMR Data Supplied by Toyokuni et al. and Caille et al.

\begin{tabular}{|c|c|c|c|c|c|c|}
\hline \multicolumn{7}{|c|}{$\delta$, Multiplicity, $J(\mathrm{~Hz})^{1}$} \\
\hline & & $\mathrm{H}-1$ & $\mathrm{H}-2$ & $\mathrm{H}-3$ & $\mathrm{H}-4$ & $\mathrm{H}-5$ \\
\hline $\mathbf{1}$ (Caille et al.) & $\alpha$-Pyranose & $5.32 \mathrm{~d}(3.6)$ & $3.79 \mathrm{dd}(10.0,3.6)$ & $3.84 \mathrm{dd}(10.0,3.0)$ & $4.23 \mathrm{~d}(3.0)$ & $4.51 \mathrm{q}(6.8)$ \\
\hline $\mathbf{1}$ (Caille et al.) & $\beta$-Pyranose & $4.64 \mathrm{~d}(8.0)$ & $3.51 \mathrm{dd}(10.0,8.0)$ & $3.63 \mathrm{dd}(10.0,3.0)$ & $4.18 \mathrm{~d}(3.0)$ & $4.10-4.25$ \\
\hline $\mathbf{1}$ (Caille et al.) & $\alpha$-Furanose & $5.26 \mathrm{~d}(4.8)$ & $4.06 \mathrm{dd}(8.0,4.8)$ & $\mathrm{NA}$ & $\mathrm{NA}$ & $4.10-4.25$ \\
\hline $\mathbf{1}$ (Caille et al.) & $\beta$-Furanose & $5.19 \mathrm{~d}(3.6)$ & $3.96-4.00$ & $\mathrm{NA}$ & $\mathrm{NA}$ & $4.10-4.25$ \\
\hline & & & & & & \\
\hline $\mathbf{1}$ (Toyokuni et al.) & $\alpha$-Pyranose & $5.32 \mathrm{~d}(3.5)$ & $3.79 \mathrm{dd}(10.5,3.5)$ & $3.84 \mathrm{dd}(10.5,3.0)$ & $4.23 \mathrm{~d}(3.0)$ & $4.51 \mathrm{q}(7.0)$ \\
\hline $\mathbf{1}$ (Toyokuni et al.) & $\beta$-Pyranose & $4.65 \mathrm{~d}(8.0)$ & $3.50 \mathrm{dd}(10.0,8.0)$ & $3.63 \mathrm{dd}(10.0,3.5)$ & $4.18 \mathrm{~d}(3.5)$ & $4.10-4.25$ \\
\hline $\mathbf{1}$ (Toyokuni et al.) & $\alpha$-Furanose & $5.26 \mathrm{~d}(5.0)$ & $4.06 \mathrm{dd}(8.0,5.0)$ & unassigned & unassigned & $4.10-4.25$ \\
\hline $\mathbf{1}$ (Toyokuni et al.) & $\beta$-Furanose & $5.20 \mathrm{~d}(3.5)$ & $3.97 \mathrm{dd}(4.0,3.5)$ & unassigned & unassigned & $4.10-4.25$ \\
\hline & & & & & & \\
\hline C5-epi-1 (Toyokuni) & $\alpha$-Pyranose & $5.03 \mathrm{~d}(3.0)$ & $3.82 \mathrm{dd}(5.5,3.0)$ & $3.93-3.96$ & $4.11 \mathrm{dd}(7.5,3.5)$ & $4.41-4.49$ \\
\hline C5-epi-1 (Toyokuni) & $\beta$-Pyranose & $5.16 \mathrm{~d}(1.0)$ & $3.81 \mathrm{dd}(4.0,1.0)$ & $4.02-4.07$ & unassigned & unassigned \\
\hline C5-epi-1 (Toyokuni) & $\alpha$-Furanose & $5.25 \mathrm{~d}(2.0)$ & $4.00 \mathrm{t}(2.0)$ & $4.18-4.21$ & $4.02-4.05$ & $4.14-4.25$ \\
\hline C5-epi-1 (Toyokuni) & $\beta$-Furanose & $5.28 \mathrm{~d}(4.5)$ & $4.05 \mathrm{dd}(6.0,4.5)$ & $4.29 \mathrm{t}(6.0)$ & $3.94 \mathrm{dd}(7.5,6.0)$ & $4.14-4.25$ \\
\hline
\end{tabular}

1. Data obtained in $\mathrm{D}_{2} \mathrm{O}$ using $500 \mathrm{MHz}$ (Toyokuni et al.) and $400 \mathrm{MHz}$ (Caille et al.) spectrometers

xxiv. Equilibrium ${ }^{13} \mathrm{C}$ NMR for 1 :

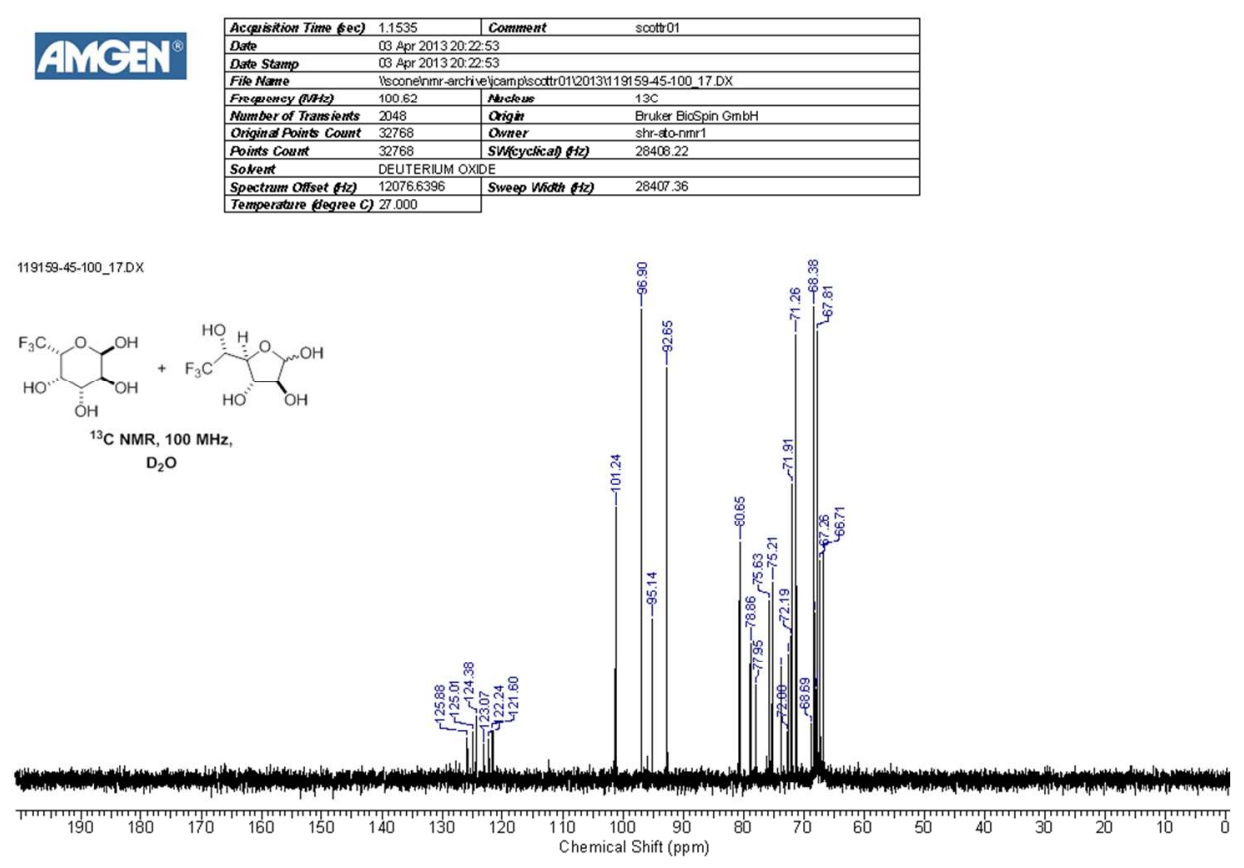


xxv. Equilibrium ${ }^{19}$ F NMR for 1:

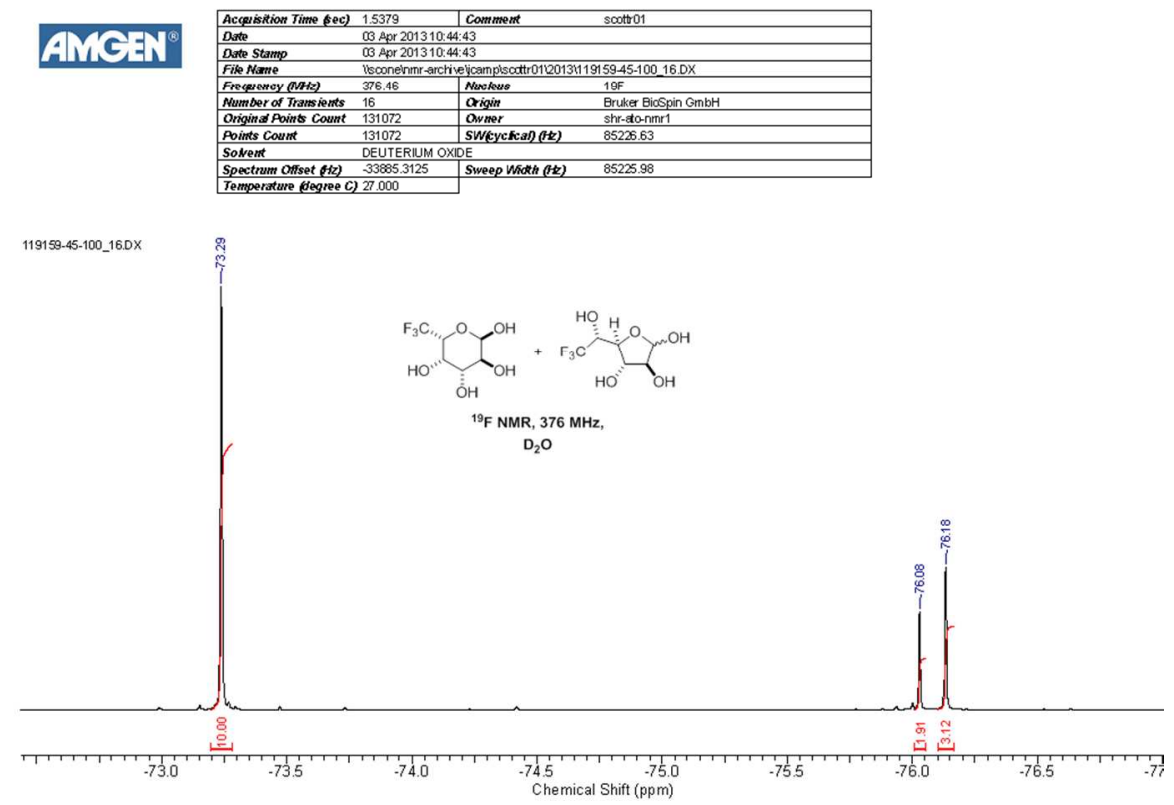

xxvi. M06-2X/6-31+G(d,p) Gibbs Free Energies, Boltzmann Populations, Total Energies, and Zeropoint Energy Corrections for Conformational Ensembles of Systems A-D Within Free Energy Window of $4 \mathrm{kcal} / \mathrm{mol}$; Gas Phase. (" $6 a$ " = species A; " $6 \mathrm{~b}$ " = species B; " $5 a$ " = species C; " $5 b$ " = species D) for $\mathbf{1}$ :

$\begin{array}{lllllll}\text { Conformer } & \Delta G(298.15 K)^{a} & \Delta G_{r e l}{ }^{b} & \text { Population } & E_{\text {tot }}{ }^{a} & E_{\text {tot, rel }}{ }^{b} & Z E^{a} \\ \text { 6a_confs_0106 } & -909.2844300 & 0 & 0.304726253 & -909.4190352 & 0 & 0.173296 \\ \text { 6a_confs_0051 } & -909.2840550 & 0.235 & 0.204953178 & -909.4182016 & 0.523 & 0.172901 \\ \text { 6a_confs_0023 } & -909.2839910 & 0.275 & 0.191573142 & -909.4186949 & 0.214 & 0.173208 \\ \text { 5a_confs_0182 } & -909.2828890 & 0.967 & 0.059579656 & -909.4144954 & 2.849 & 0.172064 \\ \text { 5b_confs_0112 } & -909.2823770 & 1.288 & 0.034658096 & -909.416065 & 1.864 & 0.172953 \\ \text { 5b_confs_1170 } & -909.2821140 & 1.453 & 0.0262337 & -909.4156176 & 2.145 & 0.1727 \\ \text { 6a_confs_0067 } & -909.2820660 & 1.483 & 0.024938454 & -909.4161172 & 1.831 & 0.172841 \\ \text { 6b_confs_0059 } & -909.2820220 & 1.511 & 0.023787319 & -909.4153033 & 2.342 & 0.172276 \\ \text { 6a_confs_0079 } & -909.2819800 & 1.537 & 0.022766039 & -909.4159714 & 1.923 & 0.172853 \\ \text { 5b_confs_0242 } & -909.2817430 & 1.686 & 0.017703957 & -909.4146992 & 2.721 & 0.172562 \\ \text { 6a_confs_0475 } & -909.2814850 & 1.848 & 0.013468654 & -909.4156143 & 2.147 & 0.172892 \\ \text { 6b_confs_0064 } & -909.2811390 & 2.065 & 0.009338196 & -909.4145073 & 2.841 & 0.172356 \\ \text { 6a_confs_0092 } & -909.2808960 & 2.218 & 0.007212965 & -909.4149124 & 2.587 & 0.172899 \\ \text { 6a_confs_0563 } & -909.2807660 & 2.299 & 0.006291306 & -909.4146889 & 2.727 & 0.172701 \\ \text { 5a_confs_0583 } & -909.2806400 & 2.378 & 0.005505968 & -909.4127008 & 3.975 & 0.171956 \\ \text { 5b_confs_0127 } & -909.2804630 & 2.489 & 0.004565314 & -909.4133569 & 3.563 & 0.172291 \\ \text { 5a_confs_0511 } & -909.2804420 & 2.502 & 0.004466235 & -909.4125419 & 4.074 & 0.172046 \\ \text { 5a_confs_0096 } & -909.2804370 & 2.506 & 0.004436184 & -909.4125268 & 4.084 & 0.172066 \\ \text { 6a_confs_0097 } & -909.2798680 & 2.863 & 0.002428445 & -909.4135201 & 3.461 & 0.172593 \\ \text { 6a_confs_0025 } & -909.2797990 & 2.906 & 0.002258443 & -909.412555 & 4.066 & 0.172239 \\ \text { 6a_confs_0146 } & -909.2797910 & 2.911 & 0.002239465 & -909.4139538 & 3.189 & 0.172904 \\ \text { 6a_confs_0093 } & -909.2797490 & 2.937 & 0.002143316 & -909.4136472 & 3.381 & 0.172701 \\ \text { 6a_confs_0132 } & -909.2797240 & 2.953 & 0.002086211 & -909.4125563 & 4.065 & 0.172257 \\ \text { 6b_confs_0053 } & -909.2794300 & 3.137 & 0.001529277 & -909.4127066 & 3.971 & 0.172278 \\ \text { 6a_confs_0105 } & -909.2794160 & 3.146 & 0.001506222 & -909.4134613 & 3.498 & 0.172768\end{array}$




$\begin{array}{lllllll}\text { 5a_confs_0601 } & -909.2793610 & 3.181 & 0.001419822 & -909.4114728 & 4.745 & 0.172068 \\ \text { 5a_confs_0081 } & -909.2792930 & 3.223 & 0.001322659 & -909.4121741 & 4.305 & 0.172561 \\ \text { 5b_confs_0372 } & -909.2791950 & 3.285 & 0.001191247 & -909.412721 & 3.962 & 0.172944 \\ \text { 5a_confs_0007 } & -909.2791580 & 3.308 & 0.00114589 & -909.4123356 & 4.204 & 0.172951 \\ \text { 5a_confs_0112 } & -909.2791580 & 3.308 & 0.00114589 & -909.4108587 & 5.131 & 0.171804 \\ \text { 5a_confs_0719 } & -909.2791060 & 3.341 & 0.001083812 & -909.4104659 & 5.377 & 0.1717 \\ \text { 6a_confs_1150 } & -909.2790870 & 3.353 & 0.001062081 & -909.4124854 & 4.11 & 0.172395 \\ \text { 5b_confs_0928 } & -909.2790300 & 3.389 & 0.00099947 & -909.4095688 & 5.94 & 0.171604 \\ \text { 6a_confs_0107 } & -909.2789020 & 3.469 & 0.000873232 & -909.4125729 & 4.055 & 0.172525 \\ \text { 5a_confs_0791 } & -909.2788850 & 3.479 & 0.000858617 & -909.4103438 & 5.454 & 0.171953 \\ \text { 6a_confs_0103 } & -909.2788420 & 3.506 & 0.000820368 & -909.412422 & 4.15 & 0.172496 \\ \text { 5b_confs_0804 } & -909.2787890 & 3.54 & 0.000774616 & -909.4110826 & 4.99 & 0.172071 \\ \text { 5b_confs_0792 } & -909.2787750 & 3.549 & 0.000762939 & -909.4110821 & 4.991 & 0.172079 \\ \text { 5a_confs_0399 } & -909.2787090 & 3.59 & 0.000711929 & -909.4106824 & 5.241 & 0.171959 \\ \text { 6b_confs_0141 } & -909.2786570 & 3.623 & 0.00067336 & -909.4118361 & 4.517 & 0.172206 \\ \text { 6b_confs_0119 } & -909.2786520 & 3.626 & 0.00066996 & -909.4116666 & 4.624 & 0.172181 \\ \text { 5b_confs_0373 } & -909.2786230 & 3.644 & 0.000649912 & -909.4116509 & 4.634 & 0.172829 \\ \text { 6b_confs_0143 } & -909.2785140 & 3.712 & 0.000579443 & -909.411802 & 4.539 & 0.172382 \\ \text { 6b_confs_0448 } & -909.2784150 & 3.774 & 0.000521873 & -909.4111627 & 4.94 & 0.171999 \\ \text { 6b_confs_1018 } & -909.2783980 & 3.785 & 0.000512273 & -909.4116437 & 4.638 & 0.172285 \\ \text { 6b_confs_0023 } & -909.2783400 & 3.821 & 0.000482074 & -909.4121679 & 4.309 & 0.172572 \\ \text { 5a_confs_0021 } & -909.2783370 & 3.823 & 0.000480449 & -909.4114407 & 4.766 & 0.172737 \\ \text { 5a_confs_0440 } & -909.2782800 & 3.859 & 0.000452126 & -909.4112737 & 4.87 & 0.172853 \\ \text { 5a_confs_0367 } & -909.2781870 & 3.917 & 0.000409964 & -909.4096491 & 5.89 & 0.171778\end{array}$

${ }^{a}$ Hartrees. ${ }^{b} \mathrm{kcal} / \mathrm{mol}$. " Normalized based on Boltzmann distribution at 298.15 using Gibbs free energy window of $<4 \mathrm{kcal} / \mathrm{mol}$. 


\section{M06-2X/6-31+G(d,p) Cartesian Coordinates (Gas Phase) for Systems A-D Within Free Energy}

\section{Window of $4 \mathrm{kcal} / \mathrm{mol}$. ("6a" = species $A$; "6b" = species B; “5a" = species C; "5b" = species}

D).

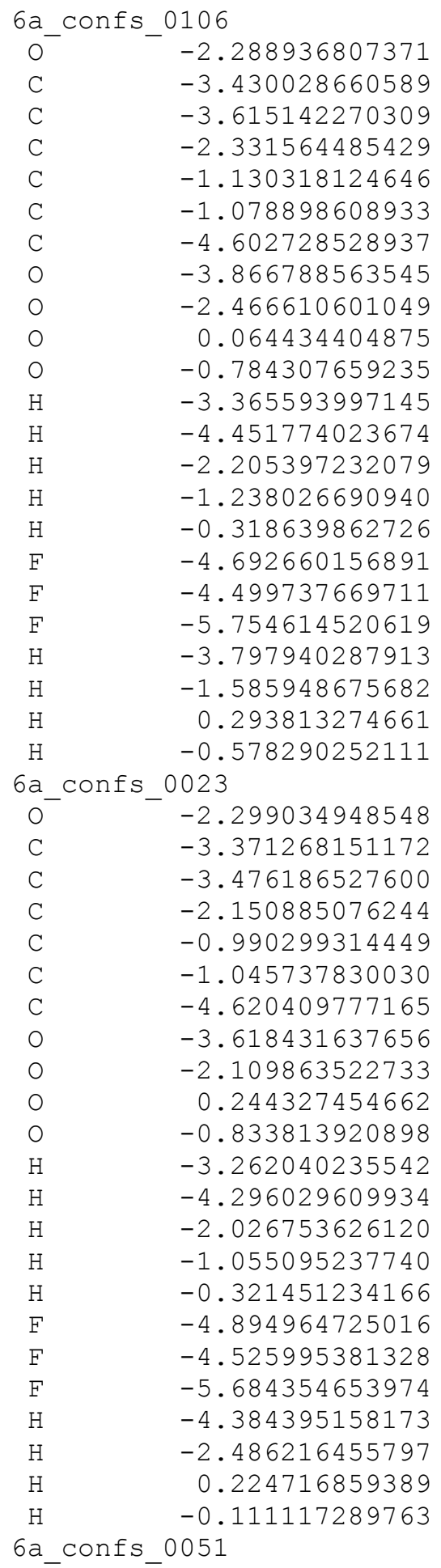

5.797236936804

4.984569674649

3. 943659748877

3.119604555696

4.039331171067

5.091075396186

5.947218592292

4. 592440801921

2. 243035469593

3. 288560988782

4. 395885204743

4.456215460084

3. 286509784801

2. 552953662881

4. 553969738529

5.848386978658

6.810519640641

6.658834328203

5.254573390746

3. 922907203082

1. 909642227039

3. 029631775386

5.025837269338

5.870566028808

4. 980798469784

3. 949095310628

3. 194342951358

4. 182324461525

5. 237298473059

5.832384157155

4. 516998265072

2. 269313185236

3. 508043459496

4. 642346195642

4. 442674074723

3. 249951144381

2. 615899560631

4. 693572661101

6.037187152955

6.534525236587

6.700484121648

5.040591869459

5.101146197834

2. 709571648863

2. 730275228758

4. 005310145299
0.180539080962

0.373151375574

$-0.728386078701$

$-0.809698246970$

$-1.009367555593$

0.095667461222

0.499704468056

$-1.949315507058$

$-1.908792071581$

$-1.072205557625$

1.284689997548

1.335065278032

$-0.449898371798$

0.125841026267

$-1.968378928779$

$-0.117774048006$

$-0.506419268188$

1. 637194358479

0.566395673833

$-2.644441450174$

$-2.129833564035$

$-0.167939505428$

1. 985701433962

0.196351234522

0.390178953810

$-0.742890427023$

$-0.792652334061$

$-0.918026880652$

0.189393286205

0.531728704062

$-2.030715058743$

$-1.858509489386$

$-0.773904384229$

1. 442019780211

1. 342502207204

$-0.527737263758$

0.129343899301

$-1.887548337027$

0.003970618680

$-0.587624197818$

1.536255873906

0.763263361861

$-2.073109601049$

$-2.635019318509$

$-1.348295096696$

1. 338424469187 


\begin{tabular}{|c|c|c|c|}
\hline \multirow{2}{*}{\multicolumn{4}{|c|}{3}} \\
\hline & & & \\
\hline $\mathrm{C}$ & -3.333299367842 & 4.953763604891 & 0.345906988114 \\
\hline $\mathrm{C}$ & -3.390064482349 & 3.954281198138 & -0.809489843653 \\
\hline C & -2.030706571251 & 3.257002007667 & -0.892301915755 \\
\hline $\mathrm{C}$ & -0.902296359535 & 4.284199923580 & -0.980472651031 \\
\hline C & -1.010376441538 & 5.315604988223 & 0.144108664942 \\
\hline C & -4.604433469948 & 5.775027768804 & 0.498506594191 \\
\hline 0 & -3.677802390487 & 4.560565280785 & -2.057977864519 \\
\hline 0 & -1.963953088988 & 2.383916386516 & -1.999126783826 \\
\hline 0 & 0.355444814850 & 3.656732906183 & -0.844911907182 \\
\hline 0 & -0.790375263881 & 4.713468606393 & 1.388475520073 \\
\hline $\mathrm{H}$ & -3.185825569824 & 4.415331039287 & 1.290516657056 \\
\hline $\mathrm{H}$ & -4.172778598542 & 3.210494505257 & -0.628422367116 \\
\hline $\mathrm{H}$ & -1.879028574288 & 2.643702261760 & 0.003697408662 \\
\hline $\mathrm{H}$ & -0.971395612304 & 4.814190788834 & -1.942263318662 \\
\hline $\mathrm{H}$ & -0.322123324445 & 6.151566750867 & -0.019953437792 \\
\hline $\mathrm{F}$ & -5.674358857101 & 4.965043787764 & 0.546928199547 \\
\hline $\mathrm{F}$ & -4.782102252195 & 6.628653212350 & -0.521350941875 \\
\hline $\mathrm{F}$ & -4.585508167460 & 6.494671015874 & 1.625052738716 \\
\hline $\mathrm{H}$ & -3.420589292911 & 5.492907109375 & -2.036703685216 \\
\hline $\mathrm{H}$ & -2.395887559567 & 2.833224953111 & -2.74161070341 \\
\hline $\mathrm{H}$ & 0.368532380236 & 2.889577586632 & -1.433967250295 \\
\hline $\mathrm{H}$ & -0.043843630754 & 4.104148428086 & 1.286488670275 \\
\hline \multicolumn{4}{|c|}{ 6a_confs_0067 } \\
\hline 0 & -2.296456601540 & 5.815168369639 & 0.106595435322 \\
\hline $\mathrm{C}$ & -3.362858503701 & 4.913300838322 & 0.282154615691 \\
\hline $\mathrm{C}$ & -3.436232630094 & 3.861229269152 & -0.834081268785 \\
\hline $\mathrm{C}$ & -2.087239817943 & 3.142092605996 & -0.886288140703 \\
\hline C & -0.959564887090 & 4.167760529956 & -1.013796341404 \\
\hline $\mathrm{C}$ & -1.029060130281 & 5.168908935594 & 0.134530112530 \\
\hline $\mathrm{C}$ & -4.624634220622 & 5.748895032759 & 0.399752418745 \\
\hline 0 & -3.610877054078 & 4.407638125927 & -2.127882105640 \\
\hline 0 & -2.031167713694 & 2.231565124628 & -1.964107762559 \\
\hline 0 & 0.298504739762 & 3.545076266294 & -1.004714091672 \\
\hline 0 & -0.838315749679 & 4.486756921437 & 1.335241196970 \\
\hline $\mathrm{H}$ & -3.267659525426 & 4.380737264799 & 1.239723356754 \\
\hline $\mathrm{H}$ & -4.232972448657 & 3.141001533921 & -0.602448577721 \\
\hline $\mathrm{H}$ & -1.936237389354 & 2.561260887543 & 0.029587357196 \\
\hline $\mathrm{H}$ & -1.113609369013 & 4.739347603861 & -1.940735845438 \\
\hline $\mathrm{H}$ & -0.299253395699 & 5.972081056159 & -0.001140046546 \\
\hline $\mathrm{F}$ & -4.557420759787 & 6.611844580096 & 1.415001395948 \\
\hline $\mathrm{F}$ & -5.684602182441 & 4.945298398194 & 0.609722842453 \\
\hline $\mathrm{F}$ & -4.887619601201 & 6.456141757798 & -0.716219049008 \\
\hline $\mathrm{H}$ & -4.420564767625 & 4.928043136163 & -2.181687547032 \\
\hline $\mathrm{H}$ & -2.428593418182 & 2.670636826603 & -2.730702189424 \\
\hline $\mathrm{H}$ & 0.246904633178 & 2.789116256373 & -1.605231945081 \\
\hline $\mathrm{H}$ & -0.637169206832 & 5.117398678790 & 2.036026179403 \\
\hline \multicolumn{4}{|c|}{ 5b_confs_0112 } \\
\hline 0 & -6.337198225977 & 3.671240159341 & 0.46454105181 \\
\hline $\mathrm{C}$ & -5.841938348861 & 3.936291593681 & -0.856464258244 \\
\hline $\mathrm{C}$ & -5.351797697167 & 2.574524451060 & -1.423154120225 \\
\hline C & -5.951212075069 & 1.558477400299 & -0.43400180764 \\
\hline C & -5.925289502963 & 2.378122188782 & 0.854781207018 \\
\hline $\mathrm{C}$ & -6.996353980988 & 4.556111362914 & -1.650730246118 \\
\hline $\mathrm{C}$ & -7.235656330003 & 6.020949270396 & -1.29443350895 \\
\hline 0 & -8.189300526411 & 3.836422375122 & -1.459813169236 \\
\hline $\mathrm{H}$ & 94019061446 & 4.628371123178 & -0 . \\
\hline
\end{tabular}




\begin{tabular}{|c|c|c|c|}
\hline 0 & -3.948414609658 & 2.509353222738 & -1.494290712028 \\
\hline 0 & -7.258536612007 & 1.165585538302 & -0.762156916664 \\
\hline 0 & -4.591272759599 & 2.396349612361 & 1.306578260583 \\
\hline $\mathrm{H}$ & -5.725400447906 & 2.391467430570 & -2.434959600854 \\
\hline $\mathrm{H}$ & -5.330637081596 & 0.663128711935 & -0.361171558913 \\
\hline $\mathrm{H}$ & -6.620002613275 & 2.032628538148 & 1.624989272612 \\
\hline $\mathrm{H}$ & -6.762075109320 & 4.534882239419 & -2.719521344216 \\
\hline $\mathrm{F}$ & -7.690974820355 & 6.151449830473 & -0.037466417375 \\
\hline $\mathrm{F}$ & -8.141834042234 & 6.575378518522 & -2.108344663405 \\
\hline $\mathrm{F}$ & -6.103465570348 & 6.735714264862 & -1.397947003243 \\
\hline $\mathrm{H}$ & -8.384280658946 & 3.874234946755 & -0.508889357141 \\
\hline $\mathrm{H}$ & -3.603235573717 & 2.506267136171 & -0.589174208099 \\
\hline $\mathrm{H}$ & -7.730208158517 & 1.903587444679 & -1.178508363124 \\
\hline $\mathrm{H}$ & -4.520696193639 & 3.001662640294 & 2.055434104133 \\
\hline \multicolumn{4}{|c|}{ 6a confs 0079} \\
\hline $0^{-}$ & -2.296364383364 & 5.886265802331 & 0.172389370144 \\
\hline $\mathrm{C}$ & -3.356623305261 & 4.963279814000 & 0.338755336775 \\
\hline $\mathrm{C}$ & -3.390841121277 & 3.956791239511 & -0.811063975789 \\
\hline $\mathrm{C}$ & -2.027555048407 & 3.258141795507 & -0.878645339374 \\
\hline $\mathrm{C}$ & -0.903959042348 & 4.292386729590 & -0.943449914007 \\
\hline $\mathrm{C}$ & -1.014092373281 & 5.263000398868 & 0.227414630081 \\
\hline $\mathrm{C}$ & -4.619656759484 & 5.801957029187 & 0.453609036018 \\
\hline 0 & -3.675641217321 & 4.556533721962 & -2.063849871309 \\
\hline 0 & -1.952263490461 & 2.407104454780 & -2.002401606194 \\
\hline 0 & 0.359646797109 & 3.686218320689 & -0.931792394582 \\
\hline 0 & -0.843650860074 & 4.558910664869 & 1.414679934781 \\
\hline $\mathrm{H}$ & -3.253289220012 & 4.418040700020 & 1.285856036750 \\
\hline $\mathrm{H}$ & -4.175136937137 & 3.213498159791 & -0.635938795564 \\
\hline $\mathrm{H}$ & -1.878990726191 & 2.633185389098 & 0.009053290122 \\
\hline $\mathrm{H}$ & -1.035926480409 & 4.891741315685 & -1.859503640955 \\
\hline $\mathrm{H}$ & -0.297924287811 & 6.083865825279 & 0.128534425544 \\
\hline $\mathrm{F}$ & -5.704259272736 & 5.011794274166 & 0.466309794133 \\
\hline $\mathrm{F}$ & -4.752281087903 & 6.667963961561 & -0.560811105345 \\
\hline $\mathrm{F}$ & -4.621704824722 & 6.513723702040 & 1.589215336346 \\
\hline $\mathrm{H}$ & -3.370041863381 & 5.474268810439 & -2.064395312004 \\
\hline $\mathrm{H}$ & -2.389337668517 & 2.866557560390 & -2.735700784140 \\
\hline $\mathrm{H}$ & 0.326532198153 & 2.944185218097 & -1.551174319147 \\
\hline $\mathrm{H}$ & -0.661839025163 & 5.173885112140 & 2.134609867715 \\
\hline \multicolumn{4}{|c|}{ 5b confs 1170} \\
\hline 0 & -6.177298050136 & 4.211177437767 & 0.391841041403 \\
\hline $\mathrm{C}$ & -5.907444834317 & 3.795053216325 & -0.954415688650 \\
\hline $\mathrm{C}$ & -5.485151748741 & 2.302683088741 & -0.871149942377 \\
\hline $\mathrm{C}$ & -5.795282935732 & 1.943930660510 & 0.597052395789 \\
\hline $\mathrm{C}$ & -5.578778210697 & 3.293106978415 & 1.267537446114 \\
\hline $\mathrm{C}$ & -7.171957439473 & 4.012745472702 & -1.778080336506 \\
\hline $\mathrm{C}$ & -7.591126451828 & 5.482762706061 & -1.823555442244 \\
\hline 0 & -8.242387632963 & 3.231355611040 & -1.334728599657 \\
\hline $\mathrm{H}$ & -5.081326821011 & 4.380440124447 & -1.372652292620 \\
\hline 0 & -4.132330409131 & 2.100517369286 & -1.196660070052 \\
\hline 0 & -7.155730811040 & 1.630803212662 & 0.811332177952 \\
\hline 0 & -4.184679218215 & 3.488203076373 & 1.341214309858 \\
\hline $\mathrm{H}$ & -6.071069652524 & 1.686152045192 & -1.559168667240 \\
\hline $\mathrm{H}$ & -5.119811205758 & 1.168597161372 & 0.973348324868 \\
\hline $\mathrm{H}$ & -6.065894093218 & 3.398628492800 & 2.240384315059 \\
\hline $\mathrm{H}$ & -6.959430771509 & 3.733496138240 & -2.817011959557 \\
\hline $\mathrm{F}$ & -8.069548613462 & 5.919402992457 & -0.653793607915 \\
\hline $\mathrm{F}$ & -8.547696384926 & 5.674746215614 & -2.744184781609 \\
\hline
\end{tabular}




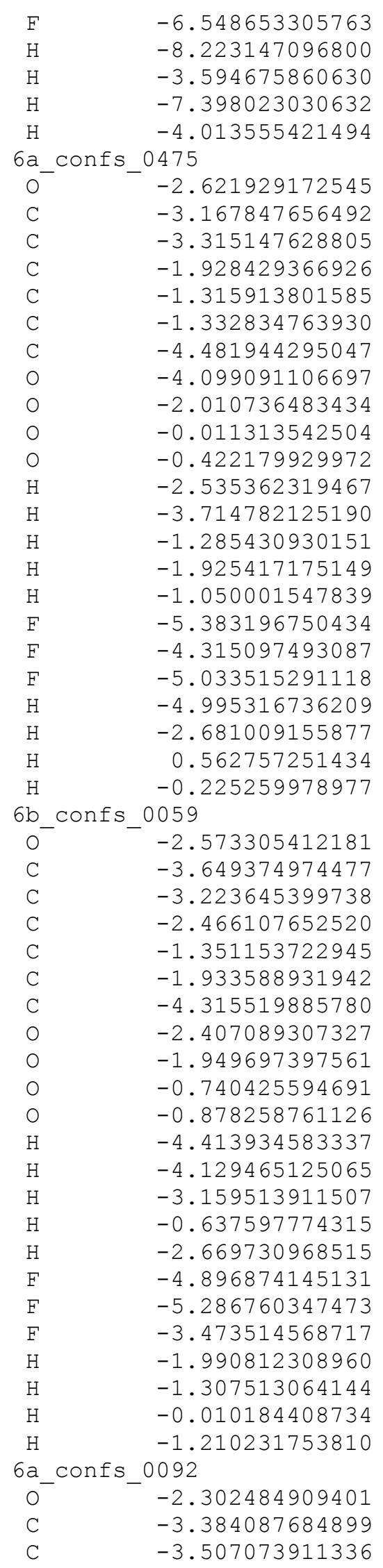

6.267897022716

3.205756063602

2. 562757413252

0.857571962696

4.388215537731

5.610783238342

5. 432522215372

3. 949628802578

3.299297422663

3.552853576373

5.050721604156

6.192151163191

3. 223336578188

1. 932828410403

3.050430509348

5.656843317475

5.910143701778

3.853857008252

3.750080457762

3.038799694456

5.222071904354

5.694251112055

7. 483732352262

6.113321431688

3.574800589260

1. 543053137780

3.562782540874

6.552709231389

5.517635453395

5.114548701923

4.179168144749

2.999403785802

3. 501127992324

4.422578317921

6.394656405560

4.880738685328

2.239784285973

2. 361471433182

4.895124982452

4.592799529855

3.803367079114

2.391192374499

4.091810436852

3.880263217270

7.035295677021

6.093860380234

7.244397946834

4.223638798436

1. 614307196087

2. 652528777702

5.508100397485

5.804833199078

4.939497021802

3. 862225977796
$-2.161588099943$

$-0.363879185962$

$-0.536878394790$

0.287933119543

1.646103938536

$-1.198746742149$

0.087119725127

0.445015256237

0.382256506013

$-1.004672717044$

$-1.329444834866$

0.108096979210

$-0.481206652308$

0.686762929242

$-1.128090270916$

$-0.445268026847$

0.849941647920

1.463900383794

1.147900568122

$-1.754094829880$

$-2.371779779950$

$-0.760328737551$

$-0.183809650499$

1.332835624396

$-0.530765577917$

0.108353867979

$-0.542887119512$

$-0.743088548600$

0.479005418150

$-0.334398967034$

$-1.468867770668$

$-0.856923634099$

0.045210376780

1.105838402637

$-0.823228886897$

$-2.369618372307$

$-1.927960754822$

0.612940639577

1. 867354770708

0.266177729896

$-1.966740868103$

$-0.253467646560$

$-0.545457695096$

1. 728686796817

0.207437931083

$-1.704031064402$

$-1.399835160758$

$-2.945064931501$

$-1.565654175580$

1.173688459088

2.535109403091

0.062881900060

0.358548293604

$-0.713490072689$ 


\begin{tabular}{|c|c|}
\hline & \\
\hline $\mathrm{C}$ & -2.196823854682 \\
\hline $\mathrm{C}$ & -1.006133218649 \\
\hline $\mathrm{C}$ & -1.050421135075 \\
\hline $\mathrm{C}$ & -4.606507194456 \\
\hline 0 & -3.778562232902 \\
\hline 0 & -2.265648203838 \\
\hline 0 & 0.236449448584 \\
\hline 0 & -0.793645008576 \\
\hline $\mathrm{H}$ & -3.246892492072 \\
\hline $\mathrm{H}$ & -4.331085754525 \\
\hline $\mathrm{H}$ & -2.068201493156 \\
\hline $\mathrm{H}$ & -1.078733266177 \\
\hline $\mathrm{H}$ & -0.315473824107 \\
\hline $\mathrm{F}$ & -4.512860133006 \\
\hline $\mathrm{F}$ & -5.715424386770 \\
\hline $\mathrm{F}$ & -4.768623584678 \\
\hline $\mathrm{H}$ & -3.441571217326 \\
\hline $\mathrm{H}$ & -2.607290643716 \\
\hline $\mathrm{H}$ & 0.363999129796 \\
\hline $\mathrm{H}$ & -0.581904429036 \\
\hline & 0242 \\
\hline 0 & -6.208461777491 \\
\hline $\mathrm{C}$ & -5.823265524579 \\
\hline $\mathrm{C}$ & -5.443083108171 \\
\hline $\mathrm{C}$ & -5.934845857958 \\
\hline $\mathrm{C}$ & -5.743315296230 \\
\hline $\mathrm{C}$ & -6.971156965427 \\
\hline $\mathrm{C}$ & -7.356976954280 \\
\hline 0 & -8.088240164740 \\
\hline $\mathrm{H}$ & -4.946524884847 \\
\hline 0 & -4.062611696205 \\
\hline 0 & -7.276952095618 \\
\hline 0 & -4.359109995807 \\
\hline $\mathrm{H}$ & -5.956823696593 \\
\hline $\mathrm{H}$ & -5.327907452990 \\
\hline $\mathrm{H}$ & -6.331476173198 \\
\hline $\mathrm{H}$ & -6.635671059069 \\
\hline $\mathrm{F}$ & -6.314710397416 \\
\hline $\mathrm{F}$ & -7.812647697933 \\
\hline $\mathrm{F}$ & -8.338925955858 \\
\hline $\mathrm{H}$ & -8.796935578529 \\
\hline $\mathrm{H}$ & -3.602976719994 \\
\hline $\mathrm{H}$ & -7.809176786458 \\
\hline $\mathrm{H}$ & -4.197204160610 \\
\hline & 0563 \\
\hline 0 & -1.994507419492 \\
\hline $\mathrm{C}$ & -2.935091517168 \\
\hline $\mathrm{C}$ & -3.869181805065 \\
\hline $\mathrm{C}$ & -2.964209632158 \\
\hline $\mathrm{C}$ & -1.936004089058 \\
\hline $\mathrm{C}$ & -1.147297885072 \\
\hline $\mathrm{C}$ & -3.666074462863 \\
\hline 0 & -4.509591134633 \\
\hline 0 & -3.734518333424 \\
\hline 0 & -1.071435005624 \\
\hline 0 & -0.326870417551 \\
\hline $\mathrm{H}$ & -2.427965758877 \\
\hline
\end{tabular}

3.060823502450

4.012765165885

5.142349203615

5.835876701389

4.401065824762

2.098528922815

3.364189943565

4.554885063580

4. 462093597928

3.182100127782

2. 513815648367

4.464527459190

5.916701410037

6.631634681662

5.093866265661

6.626178768340

5.306128550282

2.539683381501

3. 023586413283

5.236543169230

3.853574119530

3.909540791101

2. 458746884496

1.629518413171

2. 640410231682

4. 482742124247

5.902079708075

3.636812072192

4.555754798629

2. 324134919260

1.238097414870

2.706022417218

2.143685579724

0.732373370946

2.436033486524

4.553495075409

6.736169506268

6.005521882736

6.341022942120

3. 917708363980

2. 448170197557

1.998341902102

3.423043798162

5.256887361970

5.488367834275

4.280088719362

3.125022257879

2. 879800413519

4.158477846562

6.765368630030

3.896567705728

1. 977383544266

1.819330260310

4.378763875796

5.704102410575
$-0.768223472083$

$-0.959241083935$

0.075075036145

0.479046585841

$-1.996252360639$

$-1.786344043698$

$-0.914866084560$

1.322179715859

1.337566318527

$-0.474925266219$

0.174680991685

$-1.955507251427$

$-0.162608644873$

1. 554173661471

0.617367606176

$-0.590604674997$

$-2.048873874664$

$-2.577556176217$

$-0.019235839481$

1.970908736115

0.444921625524

$-0.930096936784$

$-1.345086797534$

$-0.140617600642$

0.986550150255

$-1.746582662400$

$-1.341486648780$

$-1.617060769116$

$-1.056178194982$

$-1.583731143407$

$-0.244106634803$

1.257165310801

$-2.256742335955$

$-0.005196918290$

1.884691975246

$-2.791387855908$

$-1.481320912703$

$-0.097038757580$

$-2.163370815370$

$-2.208903694233$

$-0.740288884705$

$-0.527102311069$

1.882970812434

$-1.016594779592$

0.010249469582

0.202629669747

0.622661170366

$-0.482417171845$

$-0.774315508412$

$-0.361161060868$

$-0.988516671985$

0.858619186395

$-0.132192359020$

0.349028326895

0.961905839755 


\begin{tabular}{|c|c|c|c|}
\hline $\mathrm{H}$ & -4.590000942345 & 4.491736327245 & 1.004785499859 \\
\hline $\mathrm{H}$ & -2.433223582206 & 3.427534129478 & 1.541146651087 \\
\hline $\mathrm{H}$ & -2.468692828852 & 2.576898691075 & -1.387321571671 \\
\hline $\mathrm{H}$ & -0.550188055341 & 4.053160864101 & -1.684679014592 \\
\hline $\mathrm{F}$ & -4.537782877076 & 7.087750854828 & 0.611700317125 \\
\hline $\mathrm{F}$ & -4.372084255072 & 6.650294396149 & -1.500478278944 \\
\hline $\mathrm{F}$ & -2.825355663007 & 7.792433321931 & -0.513193035452 \\
\hline $\mathrm{H}$ & -5.092492741213 & 4.598036052019 & -1.299916147674 \\
\hline $\mathrm{H}$ & -3.137013574393 & 1.217791637759 & 0.826810072783 \\
\hline $\mathrm{H}$ & -0.432755764131 & 2.162758446076 & 0.509039342247 \\
\hline $\mathrm{H}$ & 0.318337744622 & 5.067444419068 & 0.149210054213 \\
\hline \multicolumn{4}{|c|}{ 6b confs 0064} \\
\hline 0 & -2.545931223632 & 5.511460524396 & 0.464629223048 \\
\hline C & -3.636264017894 & 5.101559302226 & -0.328235429759 \\
\hline $\mathrm{C}$ & -3.240528566262 & 4.149703005562 & -1.459165863358 \\
\hline $\mathrm{C}$ & -2.474953516970 & 2.975743150449 & -0.849256391940 \\
\hline $\mathrm{C}$ & -1.331427903133 & 3.497121513331 & 0.002825658010 \\
\hline $\mathrm{C}$ & -1.867972746726 & 4.436362704756 & 1.079170890026 \\
\hline $\mathrm{C}$ & -4.301018234182 & 6.381033804023 & -0.822863883838 \\
\hline 0 & -2.438610283437 & 4.835588924662 & -2.386767413941 \\
\hline 0 & -1.999403413144 & 2.190102903537 & -1.920769932941 \\
\hline 0 & -0.693709880161 & 2.374146600421 & 0.571632870844 \\
\hline 0 & -0.838948934512 & 4.955960182180 & 1.854510338904 \\
\hline $\mathrm{H}$ & -4.391287582185 & 4.594826477874 & 0.295352892439 \\
\hline $\mathrm{H}$ & -4.159313924277 & 3.774731441109 & -1.932670800565 \\
\hline $\mathrm{H}$ & -3.151359185664 & 2.386123160400 & -0.209346013215 \\
\hline $\mathrm{H}$ & -0.642493665997 & 4.070354258899 & -0.636325645443 \\
\hline $\mathrm{H}$ & -2.535646210494 & 3.891149534921 & 1.764760455510 \\
\hline $\mathrm{F}$ & -4.863033822293 & 7.039434851540 & 0.204873083199 \\
\hline $\mathrm{F}$ & -5.284013448324 & 6.076455273053 & -1.688670858513 \\
\hline $\mathrm{F}$ & -3.457542759761 & 7.216073243061 & -1.421182774579 \\
\hline $\mathrm{H}$ & -2.056391123787 & 4.169912186923 & -2.975810427283 \\
\hline $\mathrm{H}$ & -1.354210767076 & 1.562703749359 & -1.567404573147 \\
\hline $\mathrm{H}$ & -0.000711452209 & 2.683192776865 & 1.169937651078 \\
\hline $\mathrm{H}$ & -0.426827337881 & 5.674860430453 & 1.354276945467 \\
\hline \multicolumn{4}{|c|}{ 5a_confs_0182 } \\
\hline 0 & -5.002103834134 & 4.972056461829 & -1.174479673832 \\
\hline $\mathrm{C}$ & -6.184897404887 & 4.156820049703 & -1.231462535672 \\
\hline $\mathrm{C}$ & -5.895304162021 & 2.925558692552 & -0.370958449414 \\
\hline $\mathrm{C}$ & -4.365346096510 & 2.897212047879 & -0.331824090704 \\
\hline $\mathrm{C}$ & -4.033758718776 & 4.390373608159 & -0.344654631173 \\
\hline $\mathrm{C}$ & -7.373598425441 & 4.958549812284 & -0.708603131693 \\
\hline $\mathrm{C}$ & -7.724015654478 & 6.139945134585 & -1.610942566183 \\
\hline 0 & -7.174252819850 & 5.407373418925 & 0.600424170625 \\
\hline $\mathrm{H}$ & -6.355085936164 & 3.848815401615 & -2.268476324487 \\
\hline 0 & -6.488532841083 & 1.796674479577 & -0.965658648300 \\
\hline 0 & -3.818794189542 & 2.186281712012 & 0.742876666432 \\
\hline 0 & -4.158001959357 & 4.850631463243 & 0.987235548099 \\
\hline $\mathrm{H}$ & -6.274267817856 & 3.080884211616 & 0.649662287859 \\
\hline $\mathrm{H}$ & -4.002456098824 & 2.434580951647 & -1.255985366862 \\
\hline $\mathrm{H}$ & -3.049147092949 & 4.640424257208 & -0.747182763482 \\
\hline $\mathrm{H}$ & -8.253309307358 & 4.303543608792 & -0.711259644056 \\
\hline $\mathrm{F}$ & -6.792072911645 & 7.101547830044 & -1.580007016244 \\
\hline $\mathrm{F}$ & -8.885889227676 & 6.696501351284 & -1.240052640332 \\
\hline $\mathrm{F}$ & -7.859927223042 & 5.742536447412 & -2.890913747562 \\
\hline $\mathrm{H}$ & -6.252789190412 & 5.698327801450 & 0.689920923164 \\
\hline $\mathrm{H}$ & -6.345011300637 & 1.033938711951 & -0.392614240118 \\
\hline
\end{tabular}




\begin{tabular}{|c|c|}
\hline $\begin{array}{l}\mathrm{H} \\
\mathrm{H}\end{array}$ & $\begin{array}{l}-3.903215856952 \\
-3.640321930407\end{array}$ \\
\hline \multicolumn{2}{|c|}{$6 a$ confs 0146} \\
\hline 0 & -2.301801650946 \\
\hline $\mathrm{C}$ & -3.405539231526 \\
\hline $\mathrm{C}$ & -3.535253338854 \\
\hline $\mathrm{C}$ & -2.203900554759 \\
\hline $\mathrm{C}$ & -1.073695149782 \\
\hline $\mathrm{C}$ & -1.059618421190 \\
\hline $\mathrm{C}$ & -4.623694804185 \\
\hline 0 & -3.853009195539 \\
\hline 0 & -2.304933021527 \\
\hline 0 & 0.115050877654 \\
\hline 0 & -0.796885935210 \\
\hline $\mathrm{H}$ & -3.324164243574 \\
\hline $\mathrm{H}$ & -4.321251934285 \\
\hline $\mathrm{H}$ & -2.017875798309 \\
\hline $\mathrm{H}$ & -1.273777898657 \\
\hline $\mathrm{H}$ & -0.324530450664 \\
\hline $\mathrm{F}$ & -4.741951998620 \\
\hline $\mathrm{F}$ & -4.567509038972 \\
\hline $\mathrm{F}$ & -5.743500431077 \\
\hline $\mathrm{H}$ & -3.746305742091 \\
\hline $\mathrm{H}$ & -1.417885468004 \\
\hline $\mathrm{H}$ & 0.818938224207 \\
\hline $\mathrm{H}$ & -0.673504794092 \\
\hline \multicolumn{2}{|c|}{$6 a$ confs 0093} \\
\hline $0^{-}$ & -2.346956513002 \\
\hline $\mathrm{C}$ & -3.487527313516 \\
\hline $\mathrm{C}$ & -3.628198478639 \\
\hline $\mathrm{C}$ & -2.337230417773 \\
\hline $\mathrm{C}$ & -1.143673178156 \\
\hline $\mathrm{C}$ & -1.139867800522 \\
\hline $\mathrm{C}$ & -4.672229987905 \\
\hline 0 & -3.849987168764 \\
\hline 0 & -2.438828700847 \\
\hline 0 & 0.019295447663 \\
\hline 0 & -0.950505717319 \\
\hline $\mathrm{H}$ & -3.448015970786 \\
\hline $\mathrm{H}$ & -4.464032388444 \\
\hline $\mathrm{H}$ & -2.231877147407 \\
\hline $\mathrm{H}$ & -1.211914463791 \\
\hline $\mathrm{H}$ & -0.370712521730 \\
\hline $\mathrm{F}$ & -4.628892546916 \\
\hline $\mathrm{F}$ & -5.820198410035 \\
\hline $\mathrm{F}$ & -4.726188179635 \\
\hline $\mathrm{H}$ & -3.793900057839 \\
\hline $\mathrm{H}$ & -1.588183579382 \\
\hline $\mathrm{H}$ & 0.755682786611 \\
\hline $\mathrm{H}$ & -0.287157691865 \\
\hline \multicolumn{2}{|c|}{$6 a$ confs 0097} \\
\hline $0^{-}$ & -2.29031538961 \\
\hline $\mathrm{C}$ & -3.381640427003 \\
\hline $\mathrm{C}$ & -3.490728689961 \\
\hline $\mathrm{C}$ & -2.190828819494 \\
\hline $\mathrm{C}$ & -0.995847972493 \\
\hline $\mathrm{C}$ & -1.03393153049 \\
\hline
\end{tabular}

2.741835536411

5.654087009821

5.846481806373

4.974615416136

3.972844314840

3.235118697894

4. 243720297197

5.179526993373

5.874251815552

4. 656945118593

2.430420546067

3. 494305623734

4. 421496763901

4.408208772502

3. 248717985882

2. 619611132734

4.859038336814

5.983416952210

6.782132564068

6.533908348892

5.128195412283

4.025905366329

2.106050174333

4.050757831484

5.015029728807

5.813352174064

4.994817441003

3.962520527964

3.149157994658

4.083049280310

5.112363039653

5.947985183365

4.622744259021

2.289832943970

3.262197734510

4.472895833575

4.466373890163

3.291657852005

2.569079997635

4.606834846943

5.877751565849

6.650443887223

5.243034091477

6.818416460790

3.956911226390

1.846953104634

3.691587993547

3.778638671250

5.801997582924

4. 915250018809

3.945913055195

3.134161589838

4.076000418368

5.156135992671
1.531456299925

1.111699574008

0.230303337813

0.375952023042

$-0.769815814576$

$-0.913575850062$

$-1.061522594844$

0.149174322902

0.531565273682

$-1.956784395636$

$-2.070482745127$

$-1.212834202913$

1.291462974625

1.314277176416

$-0.511897067374$

$-0.022687570292$

$-1.948177073771$

0.021540447707

$-0.432048676118$

1.703649308216

0.548606942439

$-2.682347500107$

$-2.276620673622$

$-1.562887503640$

2. 042049861240

0.203141423547

0.358843288611

$-0.758695991068$

$-0.816858273967$

$-0.956276426580$

0.181038347733

0.443313288145

$-1.980765372256$

$-1.933479568423$

$-0.888718804262$

1. 417186171433

1. 321028704164

$-0.514775996866$

0.111159178076

$-1.916463483591$

0.016375114903

1. 587057170832

0.450272944514

$-0.560779273374$

$-2.679948738456$

$-2.048429169098$

$-1.338497137744$

1.294572603726

0.121131586777

0.323848943755

$-0.855635298339$

$-0.856715950970$

$-0.994210284895$

0.088932457569 


\begin{tabular}{|c|c|}
\hline \multirow{2}{*}{\multicolumn{2}{|c|}{-4 598730572210}} \\
\hline & -4.598730572210 \\
\hline 0 & -3.699590260604 \\
\hline 0 & -2.220453354675 \\
\hline 0 & 0.220840688640 \\
\hline 0 & -0.750092018733 \\
\hline $\mathrm{H}$ & -3.258667799367 \\
\hline $\mathrm{H}$ & -4.336248012822 \\
\hline $\mathrm{H}$ & -2.117707719074 \\
\hline $\mathrm{H}$ & -1.047842665312 \\
\hline $\mathrm{H}$ & -0.311733186159 \\
\hline $\mathrm{F}$ & -4.739253094131 \\
\hline $\mathrm{F}$ & -4.515163099828 \\
\hline $\mathrm{F}$ & -5.714264183753 \\
\hline $\mathrm{H}$ & -3.335659956108 \\
\hline $\mathrm{H}$ & -1.319426005837 \\
\hline $\mathrm{H}$ & 0.395634784266 \\
\hline $\mathrm{H}$ & -0.580050715231 \\
\hline \multicolumn{2}{|c|}{$6 a$ confs 0105} \\
\hline 0 & -2.278053147206 \\
\hline $\mathrm{C}$ & -3.376592056541 \\
\hline $\mathrm{C}$ & -3.529145075042 \\
\hline $\mathrm{C}$ & -2.206478693256 \\
\hline $\mathrm{C}$ & -1.051314732781 \\
\hline $\mathrm{C}$ & -1.046037517585 \\
\hline $\mathrm{C}$ & -4.594013327913 \\
\hline 0 & -3.830289683672 \\
\hline 0 & -2.207427419319 \\
\hline 0 & 0.189956365068 \\
\hline 0 & -0.792648295901 \\
\hline $\mathrm{H}$ & -3.272905797334 \\
\hline $\mathrm{H}$ & -4.334465408365 \\
\hline $\mathrm{H}$ & -2.031173527313 \\
\hline $\mathrm{H}$ & -1.174696177807 \\
\hline $\mathrm{H}$ & -0.308559358014 \\
\hline $\mathrm{F}$ & -4.525120951505 \\
\hline $\mathrm{F}$ & -5.709693288368 \\
\hline $\mathrm{F}$ & -4.736104740871 \\
\hline $\mathrm{H}$ & -3.692873663076 \\
\hline $\mathrm{H}$ & -2.624488659188 \\
\hline $\mathrm{H}$ & 0.179913642818 \\
\hline $\mathrm{H}$ & -0.036588486829 \\
\hline \multicolumn{2}{|c|}{ 5b_confs_0127 } \\
\hline 0 & -6.280219398820 \\
\hline $\mathrm{C}$ & -5.800272826212 \\
\hline $\mathrm{C}$ & -5.446863434347 \\
\hline $\mathrm{C}$ & -6.021225588101 \\
\hline $\mathrm{C}$ & -5.875815075797 \\
\hline $\mathrm{C}$ & -6.881935246479 \\
\hline $\mathrm{C}$ & -7.303592332751 \\
\hline 0 & -8.012761964252 \\
\hline $\mathrm{H}$ & -4.895354122428 \\
\hline 0 & -4.064674096894 \\
\hline 0 & -7.391508893680 \\
\hline 0 & -4.510318186512 \\
\hline $\mathrm{H}$ & -5.931147833981 \\
\hline $\mathrm{H}$ & -5.459747357874 \\
\hline $\mathrm{H}$ & -6.516134724029 \\
\hline
\end{tabular}

5.807185823561

4.623757013900

2.207189322885

3. 362082561974

4.512882270708

4.356121676372

3.264964238268

2.614566744018

4.556651307257

5.951421736628

6.703330252584

6.489392400781

5.064190339092

5.517217634585

1.881020771064

3.124401760046

5.169165488471

5.820988123707

4.961207789862

3.934056296081

3.173606637419

4.147443809252

5.166999357020

5.863589988861

4.593607206510

2.337411992857

3.472403564553

4.522733555152

4.424671665450

3. 235731918578

2. 583812268781

4.684349208570

5.953367678908

6.559956783268

5.108223736284

6.734989269706

3.964125583133

1. 492597236932

2.772088731504

3. 933037597612

3.749584491630

3.923149133932

2. 503426257761

1. 589182116393

2. 490258568236

4.616800075347

5. 951424097092

3.829843152148

4.540592449972

2. 320827429357

1. 379373605810

2. 474839512615

2.291559145820

0.653369921174

2.230291987036
0.511489542666

$-2.071071280242$

$-1.908134286735$

$-0.961287534876$

1.303975739784

1.260130996014

$-0.713111268438$

0.114832020633

$-1.976654778616$

$-0.118342230278$

$-0.472462860751$

1.664497954965

0.560131685159

$-2.013368114712$

$-2.036076276064$

$-0.039298811944$

1.990498049538

0.152599655069

0.355279187188

$-0.767384089623$

$-0.886865883450$

$-1.066710626993$

0.075146936178

0.505878120273

$-1.971129824120$

$-2.039881441048$

$-1.011463360964$

1.298430088032

1. 308627380581

$-0.489036945825$

0.022323951521

$-2.015497848130$

$-0.116639107887$

1. 651121305618

0.563672799506

$-0.488196929168$

$-2.692284642410$

$-1.836170076204$

$-1.676564551206$

1.163945903061

0.434804481006

$-0.908373018750$

$-1.431471009284$

$-0.335311702781$

0.889179047200

$-1.729257106008$

$-1.111609082571$

$-1.954548330259$

$-0.900325148063$

$-1.611922245363$

$-0.620443604311$

1.240616933457

$-2.387257157147$

$-0.259089207018$

1.739327268643 


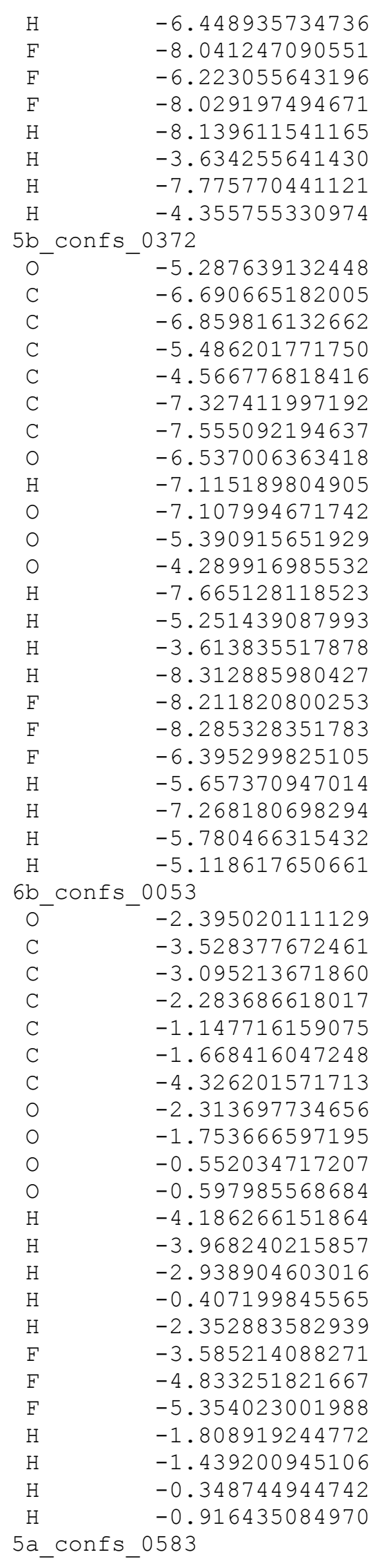

4.870759125495

6.661163323244

6.698677416595

5.805957497318

3.214115398410

2.410745864265

0.770879035425

3.149080394925

3.752902904798

3.509471109311

2.193260677041

2.009959599468

2.605846555146

4.730753054618

5.882497831610

5.180958895665

3.372944302341

1.181044956494

2.679146607707

1. 722314771568

2.240017050562

0.957333174925

2. 958098550147

4.471125473649

6.889131398376

5.480481330806

6.370688371751

5.363506237318

0.341193763635

3.565637735750

1. 325685647314

5.558281690938

5.032869538089

4.066015002842

2.950194719444

3.510641294227

4.566341098690

6.227327063959

4.695504613241

2.066084266567

2.480080954783

5.188148274513

4.502901678545

3.639452640956

2. 350786323829

4.009430434322

4.109539387880

7.065270289660

6.927261388782

5.809630403425

5.419559381886

2.607756439610

1.761856052423

5.799767061387
$-2.705176002094$

$-1.979136586567$

$-0.804378096008$

0.001171759636

$-1.215114430056$

$-0.749214408525$

0.021187097779

1. 914640547088

$-1.120659028040$

$-1.044655964751$

$-0.256530715649$

0.405146730474

$-0.672735175345$

$-0.360312154258$

$-1.335506429840$

0.710448373993

$-2.045829157007$

$-1.225956996002$

1.634666020492

$-1.707853577027$

0.486390710358

0.584860463976

$-0.276025560928$

0.038257383183

$-0.747056173280$

$-2.389325844299$

$-1.799172412562$

0.338593490363

$-0.778857792407$

1.563662001317

$-2.016548192760$

0.393494456968

$-0.254446243211$

$-1.356451995284$

$-0.695419444584$

0.165849836614

1.129789400645

$-0.758283179357$

$-2.353795716219$

$-1.657595698281$

0.911507408864

1. 728026006962

0.453585526658

$-1.860287411819$

$-0.049197091905$

$-0.481087262471$

1.866291474663

$-1.491159591349$

0.266170171874

$-1.514117838548$

$-1.955427086105$

$-2.397552165150$

0.296973121771

2.402933319263 


\begin{tabular}{|c|c|c|c|}
\hline 0 & -5.505850348888 & 4.061110122097 & -1.503475785857 \\
\hline $\mathrm{C}$ & -6.661431165167 & 3.400783782292 & -0.987580359742 \\
\hline C & -6.156422780468 & 2.233875567504 & -0.131091387156 \\
\hline $\mathrm{C}$ & -4.714335727320 & 2.063951528124 & -0.615581606438 \\
\hline $\mathrm{C}$ & -4.337032871381 & 3.511446517629 & -0.948808705811 \\
\hline C & -7.509592103846 & 4.374944031180 & -0.178793916557 \\
\hline $\mathrm{C}$ & -8.128463637269 & 5.479721222137 & -1.026402084469 \\
\hline 0 & -6.698238442014 & 4.941905467079 & 0.824437732108 \\
\hline $\mathrm{H}$ & -7.256904147001 & 3.009377783333 & -1.820353524848 \\
\hline 0 & -6.994658762698 & 1.119614613381 & -0.328990061575 \\
\hline 0 & -3.866421460877 & 1.454621524550 & 0.318337745057 \\
\hline 0 & -3.940729411291 & 4.179169727880 & 0.226446879004 \\
\hline $\mathrm{H}$ & -6.134593638255 & 2.518785275707 & 0.931075015161 \\
\hline $\mathrm{H}$ & -4.715947095099 & 1.452946995109 & -1.525565057497 \\
\hline $\mathrm{H}$ & -3.530618399785 & 3.611591490208 & -1.677743255249 \\
\hline $\mathrm{H}$ & -8.351905177109 & 3.812332598734 & 0.250027534196 \\
\hline $\mathrm{F}$ & -8.832928438437 & 6.311297200911 & -0.223183250761 \\
\hline $\mathrm{F}$ & -8.983406603749 & 4.965356400907 & -1.922682276246 \\
\hline $\mathrm{F}$ & -7.237200834145 & 6.222212854989 & -1.677229184039 \\
\hline $\mathrm{H}$ & -7.216173096478 & 5.539876818560 & 1.377749982504 \\
\hline $\mathrm{H}$ & -6.660353004655 & 0.387872005094 & 0.204000713982 \\
\hline $\mathrm{H}$ & -3.567348149452 & 2.153549750752 & 0.920403733536 \\
\hline $\mathrm{H}$ & -4.709444704614 & 4.665656721844 & 0.568001120697 \\
\hline \multicolumn{4}{|c|}{ 6a_confs_0107 } \\
\hline 0 & -2.321638191668 & 5.894053506877 & 0.188521357394 \\
\hline $\mathrm{C}$ & -3.365720221469 & 4.968363204236 & 0.364000694838 \\
\hline $\mathrm{C}$ & -3.460854886581 & 3.997667959562 & -0.827903669203 \\
\hline $\mathrm{C}$ & -2.125345301949 & 3.251604075624 & -0.857572141897 \\
\hline $\mathrm{C}$ & -0.985548234644 & 4.255494476047 & -0.985479071181 \\
\hline $\mathrm{C}$ & -1.052434429095 & 5.296417984880 & 0.136688372902 \\
\hline $\mathrm{C}$ & -4.632104655644 & 5.777805465453 & 0.576756324819 \\
\hline 0 & -3.606040997386 & 4.636272612984 & -2.071056639533 \\
\hline 0 & -1.979390777133 & 2.372819608422 & -1.952535260957 \\
\hline 0 & 0.265426114539 & 3.607920162669 & -0.850686604769 \\
\hline 0 & -0.790033733993 & 4.694865758633 & 1.376963452658 \\
\hline $\mathrm{H}$ & -3.221734209136 & 4.389818832236 & 1.287594607724 \\
\hline $\mathrm{H}$ & -4.283905664614 & 3.288427686371 & -0.641965182646 \\
\hline $\mathrm{H}$ & -2.011093430056 & 2.707277970817 & 0.092232745887 \\
\hline $\mathrm{H}$ & -1.073578536001 & 4.762443471456 & -1.953290967890 \\
\hline $\mathrm{H}$ & -0.356267153344 & 6.118478005707 & -0.058111511534 \\
\hline $\mathrm{F}$ & -5.670041932496 & 4.947003953597 & 0.790808819394 \\
\hline $\mathrm{F}$ & -4.950658470795 & 6.531383212747 & -0.496071468714 \\
\hline $\mathrm{F}$ & -4.536401847109 & 6.594402503436 & 1.624084070922 \\
\hline $\mathrm{H}$ & -4.335020438294 & 5.266968801109 & -2.046353930602 \\
\hline $\mathrm{H}$ & -2.675136171862 & 1.705994325942 & -1.944642213213 \\
\hline $\mathrm{H}$ & 0.285270474386 & 2.871798050840 & -1.476523411982 \\
\hline $\mathrm{H}$ & -0.056647305654 & 4.075418370354 & 1.245341627583 \\
\hline \multicolumn{4}{|c|}{$6 a$ confs 0132} \\
\hline 0 & -2.206941250676 & 5.837984031835 & 0.162296665243 \\
\hline $\mathrm{C}$ & -3.265586915411 & 4.924123723317 & 0.309298124537 \\
\hline $\mathrm{C}$ & -3.350557274080 & 3.964534117387 & -0.892057533681 \\
\hline $\mathrm{C}$ & -2.023944995000 & 3.203308513477 & -0.90619869808 \\
\hline $\mathrm{C}$ & -0.869233168358 & 4.194151137286 & -1.000098682556 \\
\hline $\mathrm{C}$ & -0.944492277450 & 5.224317997876 & 0.130956024116 \\
\hline $\mathrm{C}$ & -4.525824948590 & 5.747495029962 & 0.504621551734 \\
\hline 0 & -3.469147511116 & 4.612918017395 & -2.133324686648 \\
\hline 0 & -1.867806410947 & 2.334736621672 & -2.008099523207 \\
\hline
\end{tabular}




\begin{tabular}{|c|c|}
\hline 0 & 0.370758237318 \\
\hline 0 & -0.715559224997 \\
\hline $\mathrm{H}$ & -3.146575511396 \\
\hline $\mathrm{H}$ & -4.184301890622 \\
\hline $\mathrm{H}$ & -1.935832626799 \\
\hline $\mathrm{H}$ & -0.932244704871 \\
\hline $\mathrm{H}$ & -0.234027142068 \\
\hline F & -4.813550607483 \\
\hline F & -4.440657983752 \\
\hline $\mathrm{F}$ & -5.578083759589 \\
\hline $\mathrm{H}$ & -4.190049581035 \\
\hline $\mathrm{H}$ & -2.627496339977 \\
\hline $\mathrm{H}$ & 0.396071522999 \\
\hline $\mathrm{H}$ & 0.010784363898 \\
\hline \multicolumn{2}{|c|}{$6 a$ confs 0025} \\
\hline 0 & -2.206885906584 \\
\hline C & -3.265445529409 \\
\hline $\mathrm{C}$ & -3.350442259051 \\
\hline $\mathrm{C}$ & -2.023747895708 \\
\hline $\mathrm{C}$ & -0.869127573294 \\
\hline $\mathrm{C}$ & -0.944389968983 \\
\hline $\mathrm{C}$ & -4.525742818616 \\
\hline 0 & -3.469451793244 \\
\hline 0 & -1.867579242930 \\
\hline 0 & 0.370939451698 \\
\hline 0 & -0.715475769097 \\
\hline $\mathrm{H}$ & -3.146338670353 \\
\hline $\mathrm{H}$ & -4.184032906416 \\
\hline $\mathrm{H}$ & -1.935557913583 \\
\hline $\mathrm{H}$ & -0.932240444977 \\
\hline $\mathrm{H}$ & -0.233966037261 \\
\hline $\mathrm{F}$ & -4.813554323241 \\
\hline $\mathrm{F}$ & -4.440611642939 \\
\hline F & -5.577935599407 \\
\hline $\mathrm{H}$ & -4.190386855620 \\
\hline $\mathrm{H}$ & -2.629552063015 \\
\hline $\mathrm{H}$ & 0.396442505463 \\
\hline $\mathrm{H}$ & 0.010883256569 \\
\hline \multicolumn{2}{|c|}{$5 a$ confs_0511 } \\
\hline 0 & -5.709518510736 \\
\hline $\mathrm{C}$ & -5.911757992876 \\
\hline $\mathrm{C}$ & -5.229579019791 \\
\hline $\mathrm{C}$ & -4.313585969119 \\
\hline $\mathrm{C}$ & -5.130981122852 \\
\hline $\mathrm{C}$ & -7.410282816416 \\
\hline $\mathrm{C}$ & -8.077201914046 \\
\hline 0 & -8.086244511613 \\
\hline $\mathrm{H}$ & -5.421902343111 \\
\hline 0 & -4.453001188335 \\
\hline 0 & -4.046676782182 \\
\hline 0 & -6.156740207996 \\
\hline $\mathrm{H}$ & -5.971433376440 \\
\hline $\mathrm{H}$ & -3.390002879888 \\
\hline $\mathrm{H}$ & -4.549368944683 \\
\hline $\mathrm{H}$ & -7.542746623785 \\
\hline $\mathrm{F}$ & -7.399728823261 \\
\hline $\mathrm{F}$ & -8.14949502429 \\
\hline
\end{tabular}

3.529288388281

4.607689795227

4. 335499180507

3.262967141433

2. 645225230239

4.712367434901

6.039109317250

6.514474549095

6.553425683988

4. 928025504429

5.253146796528

1.747139046822

2.801429161783

3.978243579311

5.838004723206

4.924104366202

3.964118993213

3.203086644796

4.194026132365

5.224392903528

5.747431250120

4. 611992232764

2.334367298911

3.529302395016

4.608054783647

4.335750588885

3.262464624661

2.645055459615

4.712077099055

6.039184394808

6.514092935085

6.553643535362

4.927920469306

5.252190261029

1.750177845770

2.801467911728

3.978593150927

4.655180200981

4.010279136330

2. 633253793778

2.816118406567

3.770030870154

3.895838005028

5.262229287290

3.142930948551

4.607067875611

2.271147582445

1.633895466709

3.071147483427

1.859153100268

3.312833797906

4.363261573066

3.397202373328

6.021766877821

5.938998469037
$-0.848430402369$

1.370349947667

1.230035846581

$-0.724766470396$

0.038417677669

$-1.963902481626$

$-0.041556034838$

$-0.567562848921$

1. 560908306350

0.690362920041

$-2.115326630035$

$-2.086779700063$

$-1.483620018648$

1.247376647134

0.162036769398

0.309346857274

$-0.891684085416$

$-0.905880345816$

$-0.999958738099$

0.130887739817

0.504477978356

$-2.133206887145$

$-2.007673572878$

$-0.848257349028$

1.370417798764

1.230242575588

$-0.723968777799$

0.038759634695

$-1.963844839827$

$-0.041798762373$

$-0.567898371713$

1. 560551224869

0.690461957673

$-2.115270456059$

$-2.089491727215$

$-1.483465734123$

1.247617111057

0.244053286731

$-1.020212436789$

$-0.911873298130$

0.293476192804

1.159568644379

$-1.301387108809$

$-1.460458878419$

$-0.341499272420$

$-1.797112564288$

$-2.034666429275$

1.009364550137

1.829538272911

$-0.676985879089$

$-0.033103134126$

1. 869814605738

$-2.271526976506$

$-2.346154602386$

$-0.311853137291$ 


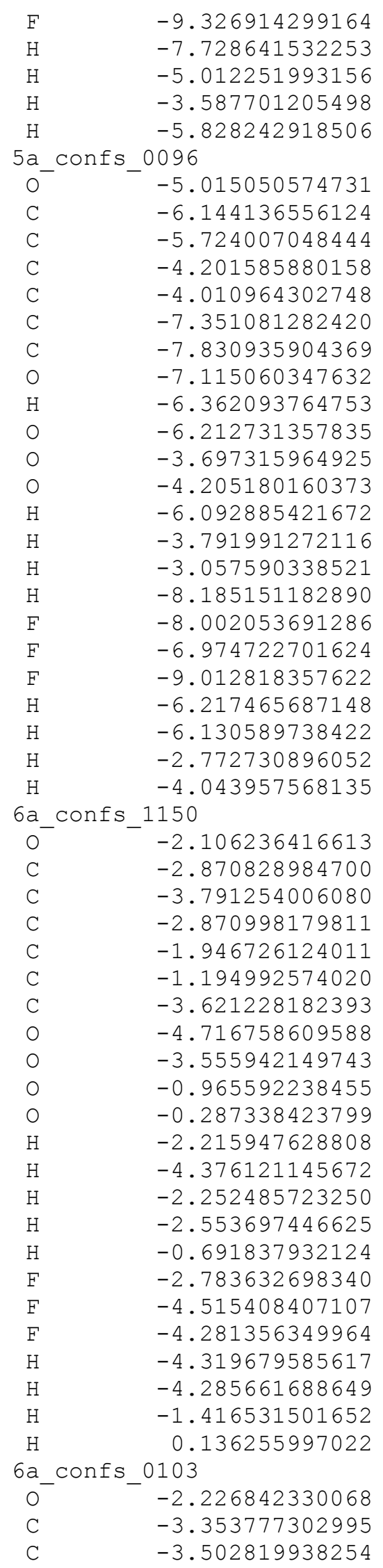

5.127226234997

3.348449744100

1.872084864377

1.010637889629

2.188266018602

5.049958478582

4.171270784926

3.022195860026

3.139985524197

4.656566774568

4.933120049919

6.019913640719

5.483943334318

3.777883244400

1.805706688834

2.610594573615

5.286240968536

3.199074864395

2. 626138743107

4.975775469398

4.225884726656

5.521506485608

7.043341264270

6.516441684761

5.859836138524

1.124882869669

2.358456875548

4. 639480955425

5.107839537756

5.447813821415

4.280239977891

3.094853695229

2.798878438810

4.060481266969

6.719587663527

3.980130221961

1. 885701244545

1.845904806265

4.458649641696

5.699175525324

4.544063451166

3.370753257312

2. 438183803488

3.903663034963

7.758352637710

7.014472677864

6.615536426364

4.156038642688

2.027238041984

1.085086984474

3. 657355200598

5.780973096889

4.952407387565

3. 894406294197
$-1.929647312880$

0.541096720858

$-2.709733509324$

0.433647372373

2.058654893802

$-1.362526385903$

$-1.273857985322$

$-0.352325316494$

$-0.337644023591$

$-0.470002348018$

$-0.728424363586$

$-1.689207611593$

0.532385967283

$-2.272256249466$

$-0.864693170343$

0.873026650521

0.774344338427

0.667251736411

$-1.215453926952$

$-0.901524541998$

$-0.637479116219$

$-2.930333590651$

$-1.775911178063$

$-1.294473725309$

0.553515677461

$-0.187298988338$

0.771267105769

1.478621045972

$-1.146104096124$

$-0.004641955739$

0.366648480114

0.691053014886

$-0.482962304439$

$-0.915363853100$

$-0.369705710413$

$-0.648216905878$

0.939078914053

$-0.127269727955$

0.072997813240

0.838868265090

1.256746320688

1.559950997282

$-1.324926815459$

$-1.875292030305$

$-0.476034113688$

0.590139347728

$-1.529250139224$

$-1.512280418687$

1. 552853262574

0.262915371750

0.415796283605

0.194682603412

0.378269818698

$-0.715667481197$ 


\begin{tabular}{|c|c|}
\hline & \\
\hline $\mathrm{C}$ & -2.183739912374 \\
\hline $\mathrm{C}$ & -1.021449923211 \\
\hline $\mathrm{C}$ & -1.004927151148 \\
\hline $\mathrm{C}$ & -4.551195155828 \\
\hline 0 & -3.801423459643 \\
\hline 0 & -2.200357971960 \\
\hline 0 & 0.211458653567 \\
\hline 0 & -0.806553197810 \\
\hline $\mathrm{H}$ & -3.295315918092 \\
\hline $\mathrm{H}$ & -4.313240914870 \\
\hline $\mathrm{H}$ & -2.011231260337 \\
\hline $\mathrm{H}$ & -1.195107328581 \\
\hline $\mathrm{H}$ & -0.232423914359 \\
\hline $\mathrm{F}$ & -4.490416119173 \\
\hline F & -5.688684426067 \\
\hline $\mathrm{F}$ & -4.643040712341 \\
\hline $\mathrm{H}$ & -3.649733720711 \\
\hline $\mathrm{H}$ & -2.546304480842 \\
\hline $\mathrm{H}$ & 0.167095498024 \\
\hline $\mathrm{H}$ & -0.589569012928 \\
\hline & 0007 \\
\hline 0 & -5.529817123803 \\
\hline $\mathrm{C}$ & -6.647337059608 \\
\hline $\mathrm{C}$ & -6.105256062880 \\
\hline $\mathrm{C}$ & -4.681152684426 \\
\hline $\mathrm{C}$ & -4.306659245385 \\
\hline $\mathrm{C}$ & -7.613321883202 \\
\hline C & -8.437155810919 \\
\hline 0 & -6.912873664245 \\
\hline $\mathrm{H}$ & -7.139039243619 \\
\hline 0 & -6.116765127572 \\
\hline 0 & -4.607756459037 \\
\hline 0 & -3.297531740998 \\
\hline $\mathrm{H}$ & -6.694297424485 \\
\hline $\mathrm{H}$ & -4.003909507815 \\
\hline $\mathrm{H}$ & -4.025285634763 \\
\hline $\mathrm{H}$ & -8.332886342202 \\
\hline $\mathrm{F}$ & -7.667581085342 \\
\hline $\mathrm{F}$ & -9.358933779717 \\
\hline F & -9.081819865294 \\
\hline $\mathrm{H}$ & -6.260853717873 \\
\hline $\mathrm{H}$ & -5.811407314177 \\
\hline $\mathrm{H}$ & -5.414435506716 \\
\hline $\mathrm{H}$ & -3.357923715921 \\
\hline & 0081 \\
\hline 0 & -4.955709573022 \\
\hline $\mathrm{C}$ & -6.135622016050 \\
\hline $\mathrm{C}$ & -5.852686804246 \\
\hline $\mathrm{C}$ & -4.324798580062 \\
\hline $\mathrm{C}$ & -3.999446622826 \\
\hline $\mathrm{C}$ & -7.317573754194 \\
\hline $\mathrm{C}$ & -7.881215285927 \\
\hline 0 & -6.944928260437 \\
\hline $\mathrm{H}$ & -6.309742669224 \\
\hline 0 & -6.478794491405 \\
\hline 0 & -3.777964055375 \\
\hline 0 & -4.079364880160 \\
\hline
\end{tabular}

3.118276981773

4.085994221072

5.068406760558

5.887433680729

4.521934807088

2.291924548124

3.414667011144

4.346930320204

4. 433805831505

3.209183237827

2. 522335611971

4.675135779783

5.828668287721

6.605884643205

5.166920319363

6.744456474184

3. 873689717273

1.419767375137

2.790210499973

4.957787112716

3.873730129847

3.209489705704

2. 313662113372

2.830699608420

3.291952184935

4.293888888742

4.907927964125

5.314030887685

2. 563915210320

0.989395815842

3.921332900847

4. 220316686134

2. 404536033016

2.057178493208

2. 458779261596

3. 864066383814

5.595700114924

5.751493232253

3.955836717760

5.651350350600

0.384681134409

4.459899622742

4.736136559706

5.076191152621

4.265204916448

3.022797901562

2. 985055531260

4.481419575924

5.076109566690

6.053002038557

5.775489951261

3.962961454716

1.909452460054

2.239663311856

4.957909577168
$-0.820169337241$

$-0.996765949932$

0.169104317455

0.475621388172

$-1.937435013077$

$-1.981158406821$

$-1.047960168321$

1. 348677112590

1.345783951397

$-0.420461876609$

0.084458766457

$-1.907780614659$

0.021884296710

1. 611417413620

0.521739397166

$-0.535793289408$

$-2.639232627263$

$-1.762886992588$

$-1.783023272251$

2.062395963691

$-1.262959039186$

$-0.693178379012$

0.445729520250

0.642412379416

$-0.775670311372$

$-0.194627776333$

$-1.322845879430$

0.474890539882

$-1.430008864821$

$-0.048403071044$

1. 535972813310

$-0.801529850941$

1. 368441906690

1.014219485250

$-1.427875666476$

0.508877739113

$-2.177823645211$

$-0.840924713974$

$-2.017493892590$

$-0.163839822211$

0.638008650190

1. 465454605175

0.018173273325

$-1.154400029860$

$-1.225370005745$

$-0.378732431591$

$-0.358133613508$

$-0.305880111214$

$-0.680384153934$

$-1.705536742111$

0.481351062777

$-2.263584005042$

$-0.963882180625$

0.694357903268

1.016408679640 


\begin{tabular}{|c|c|c|c|}
\hline $\mathrm{H}$ & -6.216591906464 & 3.169545655049 & 0.652178257626 \\
\hline $\mathrm{H}$ & -3.971007249031 & 2.555578736230 & -1.302596524228 \\
\hline $\mathrm{H}$ & -3.004062987625 & 4.744584215538 & -0.665194297704 \\
\hline $\mathrm{H}$ & -8.132628740535 & 4.393955351138 & -0.416300594260 \\
\hline $\mathrm{F}$ & -6.988611043623 & 7.013533912202 & -2.001533328424 \\
\hline $\mathrm{F}$ & -8.984610810690 & 6.658311950012 & -1.250270639163 \\
\hline $\mathrm{F}$ & -8.201323535995 & 5.422684415589 & -2.846094999961 \\
\hline $\mathrm{H}$ & -6.285822852168 & 6.435696705192 & 0.211949335957 \\
\hline $\mathrm{H}$ & -6.286261089445 & 1.130638738827 & -0.427301308230 \\
\hline $\mathrm{H}$ & -3.685751970343 & 2.833524494706 & 1.454450490412 \\
\hline $\mathrm{H}$ & -5.012880821155 & 5.028388387400 & 1.272999235916 \\
\hline \multicolumn{4}{|c|}{ 6b confs 0023} \\
\hline 0 & -2.423650995838 & 5.565862060906 & 0.388244044247 \\
\hline C & -3.529115587794 & 5.015273373766 & -0.287689593423 \\
\hline $\mathrm{C}$ & -3.070593358137 & 4.009600807542 & -1.347991430404 \\
\hline $\mathrm{C}$ & -2.244377227022 & 2.920337228977 & -0.652352891142 \\
\hline $\mathrm{C}$ & -1.149565531224 & 3.557575593275 & 0.196742682612 \\
\hline $\mathrm{C}$ & -1.744445465402 & 4.584847892563 & 1.150096236027 \\
\hline $\mathrm{C}$ & -4.305582967951 & 6.191859647801 & -0.863953068452 \\
\hline 0 & -2.287421010082 & 4.608496118877 & -2.364093514076 \\
\hline 0 & -1.676353606807 & 2.032790402588 & -1.588450094104 \\
\hline 0 & -0.469386465610 & 2.604551740282 & 0.981920012080 \\
\hline 0 & -0.768495105673 & 5.259696006982 & 1.862457903392 \\
\hline $\mathrm{H}$ & -4.213158023017 & 4.511012355629 & 0.413885283747 \\
\hline $\mathrm{H}$ & -3.937292800261 & 3.556939306487 & -1.840067709052 \\
\hline $\mathrm{H}$ & -2.891558693104 & 2.319430658485 & 0.000097433537 \\
\hline $\mathrm{H}$ & -0.444133501414 & 4.093144169752 & -0.459310324782 \\
\hline $\mathrm{H}$ & -2.468583746404 & 4.097464480867 & 1.829932927898 \\
\hline $\mathrm{F}$ & -3.531250152604 & 7.001982779160 & -1.596226452409 \\
\hline $\mathrm{F}$ & -4.854679165882 & 6.926353539934 & 0.109501080158 \\
\hline $\mathrm{F}$ & -5.298786983021 & 5.745283607082 & -1.649646475041 \\
\hline $\mathrm{H}$ & -1.839835747575 & 5.391318628942 & -2.011697706757 \\
\hline $\mathrm{H}$ & -1.383162983065 & 2.567080138234 & -2.342762982394 \\
\hline $\mathrm{H}$ & -0.189689653054 & 1.882876904915 & 0.402616688690 \\
\hline $\mathrm{H}$ & -0.102981229060 & 4.610822556955 & 2.131547949648 \\
\hline \multicolumn{4}{|c|}{ 6b_confs_0141 } \\
\hline 0 & -2.421388409577 & 5.555101315458 & 0.365312188263 \\
\hline $\mathrm{C}$ & -3.546667435257 & 5.057644743444 & -0.324103606828 \\
\hline $\mathrm{C}$ & -3.093842851599 & 4.141881759217 & -1.458872173235 \\
\hline $\mathrm{C}$ & -2.284222966628 & 2.971608448423 & -0.875261797997 \\
\hline $\mathrm{C}$ & -1.177942203170 & 3.491404862258 & 0.045282458472 \\
\hline $\mathrm{C}$ & -1.731958268663 & 4.507981168287 & 1.039799550332 \\
\hline $\mathrm{C}$ & -4.338179965097 & 6.273948720782 & -0.784121630923 \\
\hline 0 & -2.305274849419 & 4.832494214920 & -2.410989787430 \\
\hline 0 & -1.773106359318 & 2.181771236359 & -1.914690968634 \\
\hline 0 & -0.626534959014 & 2.399718229801 & 0.736054140766 \\
\hline 0 & -0.653927665385 & 5.053721423987 & 1.713038006547 \\
\hline $\mathrm{H}$ & -4.212101388594 & 4.497982605141 & 0.352970168906 \\
\hline $\mathrm{H}$ & -3.958898262934 & 3.742835403209 & -1.997156819890 \\
\hline $\mathrm{H}$ & -2.942231609479 & 2.324059055614 & -0.280708567666 \\
\hline $\mathrm{H}$ & -0.415915981735 & 4.009395425432 & -0.559606235816 \\
\hline $\mathrm{H}$ & -2.436855038389 & 4.013136901963 & 1.731450501620 \\
\hline $\mathrm{F}$ & -3.584773540030 & 7.153039085378 & -1.453212440150 \\
\hline $\mathrm{F}$ & -4.871019716997 & 6.917326459497 & 0.265201878770 \\
\hline $\mathrm{F}$ & -5.346677273209 & 5.893923851825 & -1.582948590753 \\
\hline $\mathrm{H}$ & -1.807908103448 & 5.534536175771 & -1.967210528064 \\
\hline $\mathrm{H}$ & -1.424994852181 & 2.784889041048 & -2.587557020518 \\
\hline
\end{tabular}




\begin{tabular}{|c|c|}
\hline $\mathrm{H}$ & 0.113621194767 \\
\hline $\mathrm{H}$ & -0.966499494643 \\
\hline \multicolumn{2}{|c|}{ 6b confs 0143} \\
\hline 0 & -2.487331955800 \\
\hline $\mathrm{C}$ & -3.530547614523 \\
\hline $\mathrm{C}$ & -3.027550821740 \\
\hline $\mathrm{C}$ & -2.189883764270 \\
\hline $\mathrm{C}$ & -1.114233370309 \\
\hline $\mathrm{C}$ & -1.745840241855 \\
\hline $\mathrm{C}$ & -4.339339562160 \\
\hline 0 & -2.152361800109 \\
\hline 0 & -1.592239692368 \\
\hline 0 & -0.417471085267 \\
\hline 0 & -0.833853582649 \\
\hline $\mathrm{H}$ & -4.229489068097 \\
\hline $\mathrm{H}$ & -3.885220426813 \\
\hline $\mathrm{H}$ & -2.835438519292 \\
\hline $\mathrm{H}$ & -0.436312863969 \\
\hline $\mathrm{H}$ & -2.422122359338 \\
\hline $\mathrm{F}$ & -3.609792789417 \\
\hline $\mathrm{F}$ & -4.841000576148 \\
\hline $\mathrm{F}$ & -5.367201928795 \\
\hline $\mathrm{H}$ & -2.582596799414 \\
\hline $\mathrm{H}$ & -1.255871158900 \\
\hline $\mathrm{H}$ & -0.201131562760 \\
\hline $\mathrm{H}$ & -0.134268456006 \\
\hline \multicolumn{2}{|c|}{ 6b confs 0119} \\
\hline $0^{-}$ & -2.535576507138 \\
\hline $\mathrm{C}$ & -3.632912484295 \\
\hline $\mathrm{C}$ & -3.209658030683 \\
\hline C & -2.434446144453 \\
\hline $\mathrm{C}$ & -1.297774446668 \\
\hline $\mathrm{C}$ & -1.827982254030 \\
\hline C & -4.395133519260 \\
\hline 0 & -2.312261307974 \\
\hline 0 & -1.918011276204 \\
\hline 0 & -0.716476417832 \\
\hline 0 & -0.764806891219 \\
\hline $\mathrm{H}$ & -4.340901163537 \\
\hline $\mathrm{H}$ & -4.103810688491 \\
\hline $\mathrm{H}$ & -3.112292252062 \\
\hline $\mathrm{H}$ & -0.559810215743 \\
\hline $\mathrm{H}$ & -2.526785064200 \\
\hline $\mathrm{F}$ & -5.482433828576 \\
\hline $\mathrm{F}$ & -3.658322546311 \\
\hline $\mathrm{F}$ & -4.805094813977 \\
\hline $\mathrm{H}$ & -2.687560219403 \\
\hline $\mathrm{H}$ & -1.538752649380 \\
\hline $\mathrm{H}$ & -0.515454760606 \\
\hline $\mathrm{H}$ & -1.095542517957 \\
\hline \multicolumn{2}{|c|}{ 5b confs 0373} \\
\hline 0 & -5.292589622465 \\
\hline $\mathrm{C}$ & -6.596277680504 \\
\hline $\mathrm{C}$ & -6.394299785912 \\
\hline $\mathrm{C}$ & -5.009576803381 \\
\hline $\mathrm{C}$ & -4.28635162063 \\
\hline $\mathrm{C}$ & -7.39661350616 \\
\hline
\end{tabular}

2.719861633394

5.640938238793

5.571831477033

5.017575021275

4.060517511007

2. 987354855692

3. 626312793072

4. 591585195816

6.177159640823

4. 663530519212

2.113729942491

2.634564384175

5.246513672408

4.456348985942

3.597425501144

2.367337081603

4.212509859207

4.052538260144

6.962686537899

6.947696544588

5.703309843403

5.390252720562

2.657958639568

1.931543487646

5. 614717525288

5.609530683886

5.096321285961

4.087758232399

2. 977064854230

3.548921651241

4.607537617971

6.284908008907

4.611853230653

2.041991916176

2. 520816747012

5. 215818282390

4.588229894650

3.670103867940

2. 418663574426

4.041217112373

4.147054249280

5.854999121246

7.001502126530

7.113585272293

5.373445251163

2.545633259219

1.793860950323

5.839582809732

4.179334643300

3.609315589553

2.090926658502

2.022362053127

3.163567349583

4.300382805043
1.266746250133

2. 412185024096

0.393054320897

$-0.355597128182$

$-1.447617741141$

$-0.758265821975$

0.124381430838

1. 125379651533

$-0.914353058288$

$-2.380056134048$

$-1.690441519091$

0.834346969499

1. 916523113657

0.289530016877

$-1.954070691590$

$-0.123042475757$

$-0.519576943655$

1.805424690555

$-1.728810618519$

0.048809744918

$-1.645255772223$

$-2.845331897031$

$-2.418047603212$

0.206211374275

1.361306091663

0.416074780458

$-0.282971264443$

$-1.361784511828$

$-0.658784065856$

0.194556045674

1. 150284190581

$-0.844609609928$

$-2.318218114366$

$-1.579791771405$

0.958357914342

1.776163663549

0.395793122604

$-1.844871575202$

$-0.000358087788$

$-0.455754162876$

1.872985200925

$-1.511947409483$

$-1.713435960220$

0.116420904678

$-2.774894159346$

$-2.315398826866$

0.353745421176

2.433638275621

$-1.214616780675$

$-1.197007553202$

$-1.001555944334$

$-0.350172537456$

$-1.084791805592$

$-0.067467473220$ 


\begin{tabular}{|c|c|}
\hline \multirow{2}{*}{\multicolumn{2}{|c|}{-8.107189211359}} \\
\hline & \\
\hline 0 & -6.570670207806 \\
\hline $\mathrm{H}$ & -7.092232316782 \\
\hline 0 & -6.276134027462 \\
\hline 0 & -5.059437627464 \\
\hline 0 & -3.764762527647 \\
\hline $\mathrm{H}$ & -7.162706583380 \\
\hline $\mathrm{H}$ & -4.530747382862 \\
\hline $\mathrm{H}$ & -3.471676874149 \\
\hline $\mathrm{H}$ & -8.179420083201 \\
\hline F & -7.230625160264 \\
\hline $\mathrm{F}$ & -8.845559587378 \\
\hline $\mathrm{F}$ & -8.929354138318 \\
\hline $\mathrm{H}$ & -5.816043416504 \\
\hline $\mathrm{H}$ & -7.116234714284 \\
\hline $\mathrm{H}$ & -5.526501344189 \\
\hline $\mathrm{H}$ & -4.426995777891 \\
\hline \multicolumn{2}{|c|}{ 6b confs 1018} \\
\hline 0 & -1.712727678324 \\
\hline $\mathrm{C}$ & -2.876315122880 \\
\hline $\mathrm{C}$ & -3.837321968888 \\
\hline $\mathrm{C}$ & -3.068948868980 \\
\hline $\mathrm{C}$ & -1.767952801131 \\
\hline $\mathrm{C}$ & -0.953598912384 \\
\hline $\mathrm{C}$ & -3.511180250405 \\
\hline 0 & -4.276039926146 \\
\hline 0 & -3.837337660514 \\
\hline 0 & -0.962156515181 \\
\hline 0 & 0.206910117258 \\
\hline $\mathrm{H}$ & -2.636292780373 \\
\hline $\mathrm{H}$ & -4.689408333288 \\
\hline $\mathrm{H}$ & -2.829550467776 \\
\hline $\mathrm{H}$ & -1.997378502094 \\
\hline $\mathrm{H}$ & -0.725263052606 \\
\hline $\mathrm{F}$ & -2.698132915589 \\
\hline $\mathrm{F}$ & -4.634738606746 \\
\hline $\mathrm{F}$ & -3.865437952901 \\
\hline $\mathrm{H}$ & -4.716720617091 \\
\hline $\mathrm{H}$ & -4.292682094313 \\
\hline $\mathrm{H}$ & -1.504102649459 \\
\hline $\mathrm{H}$ & 0.569377559810 \\
\hline \multicolumn{2}{|c|}{ 5a_confs_0601 } \\
\hline 0 & -6.923236379654 \\
\hline $\mathrm{C}$ & -5.849179181266 \\
\hline $\mathrm{C}$ & -5.178439938362 \\
\hline C & -5.712766495139 \\
\hline $\mathrm{C}$ & -7.106494176189 \\
\hline $\mathrm{C}$ & -6.383887182532 \\
\hline $\mathrm{C}$ & -6.982540416391 \\
\hline 0 & -7.308739444679 \\
\hline $\mathrm{H}$ & -5.147159631895 \\
\hline 0 & -3.774652297052 \\
\hline 0 & -5.815591265670 \\
\hline 0 & -7.994496844149 \\
\hline $\mathrm{H}$ & -5.535948404182 \\
\hline $\mathrm{H}$ & -5.112685077231 \\
\hline $\mathrm{H}$ & -7.528909660093 \\
\hline
\end{tabular}

5.549669539075

4.647341697463

3.794861652770

1.436223876834

2.203641963394

2.793752244596

1.623902869939

1.060581651817

3.594869696078

3.637134369983

6.463847076146

6.119159934768

5.243701384468

5.129845306409

1.462088406781

3.025723206645

2. 270766023726

5.434246915296

5.310568852377

4.236489113912

2.915393470616

3.125866550266

4.242564551889

6.690410041515

4.473012885780

1.898904279923

1.967069548346

4.510704965704

5.049283859295

4.133705421751

2.566326200077

3. 433022809887

3.983475947654

7.589380601596

6.637013007783

7.147621623806

5.326891944952

2.296572300377

1.219967815721

3.663507291478

3.771638554977

4.045243474926

2.690677026587

1.802430776465

2.390160612603

4.706822471736

6.085299736421

3.911791100099

4.724988589219

2.727341263500

0.439213915263

1.935361017266

2.296037909286

1. 949768484594

2. 222634720448
$-0.577274455059$

1. 012102023977

$-2.157952559726$

$-2.258026022044$

1.039644327147

$-2.313262472326$

$-0.375310919332$

$-0.542018077421$

$-0.500535629073$

0.314438045427

$-1.017812246111$

0.379873311687

$-1.599302715095$

0.633066648435

$-2.730492294668$

1.261341047366

$-2.789865918705$

$-0.550344102032$

0.217787234825

$-0.324127088720$

$-0.400922316439$

$-1.169862690925$

$-0.529859835094$

0.281886780547

$-1.646498781599$

$-1.004220572050$

$-1.147500286969$

$-1.234649615462$

1. 263595269094

0.361607297154

0.611516344411

$-2.200637397335$

0.522531970398

0.832430433140

1. 023542562504

$-0.933417751882$

$-1.726323381372$

$-1.761500694285$

$-1.434454155295$

$-1.529579222613$

0.696115103377

$-0.209633434719$

$-0.501230868239$

0.624427106772

0.843151726109

$-1.480492468245$

$-1.202736333286$

$-2.155890700425$

0.289672519494

$-0.605052430566$

0.292923105327

$-0.149470836996$

$-1.457992313005$

1.535700281197

1.837308352535 


\begin{tabular}{|c|c|}
\hline & \\
\hline $\mathrm{H}$ & -5.534114096733 \\
\hline $\mathrm{F}$ & -7.234042937520 \\
\hline $\mathrm{F}$ & -6.120012754031 \\
\hline $\mathrm{F}$ & -8.126800429586 \\
\hline $\mathrm{H}$ & -7.891175104679 \\
\hline $\mathrm{H}$ & -3.398563086326 \\
\hline $\mathrm{H}$ & -4.971839833493 \\
\hline $\mathrm{H}$ & -7.740725363147 \\
\hline & 0021 \\
\hline 0 & -5.459684772952 \\
\hline $\mathrm{C}$ & -6.668726505652 \\
\hline $\mathrm{C}$ & -6.220137079625 \\
\hline $\mathrm{C}$ & -4.861611986618 \\
\hline $\mathrm{C}$ & -4.483249128914 \\
\hline $\mathrm{C}$ & -7.600628422586 \\
\hline C & -7.964232506803 \\
\hline 0 & -7.094678318356 \\
\hline $\mathrm{H}$ & -7.167225964953 \\
\hline 0 & -6.084987662760 \\
\hline 0 & -4.876225695634 \\
\hline 0 & -4.463667222257 \\
\hline $\mathrm{H}$ & -6.920984455047 \\
\hline $\mathrm{H}$ & -4.121745548336 \\
\hline $\mathrm{H}$ & -3.520238881454 \\
\hline $\mathrm{H}$ & -8.540985538074 \\
\hline $\mathrm{F}$ & -8.293590363575 \\
\hline $\mathrm{F}$ & -6.945787475645 \\
\hline $\mathrm{F}$ & -9.009600140261 \\
\hline $\mathrm{H}$ & -6.279878051270 \\
\hline $\mathrm{H}$ & -5.810930700112 \\
\hline $\mathrm{H}$ & -5.540270976565 \\
\hline $\mathrm{H}$ & -4.156932602551 \\
\hline & 0440 \\
\hline 0 & -5.261068550137 \\
\hline $\mathrm{C}$ & -6.36470095615 \\
\hline $\mathrm{C}$ & -6.599292463505 \\
\hline $\mathrm{C}$ & -5.904296771492 \\
\hline $\mathrm{C}$ & -4.719690455233 \\
\hline $\mathrm{C}$ & -7.559574148537 \\
\hline $\mathrm{C}$ & -7.541723076053 \\
\hline 0 & -7.571619303227 \\
\hline $\mathrm{H}$ & -6.128728477067 \\
\hline 0 & -5.901773764953 \\
\hline 0 & -6.710928190102 \\
\hline 0 & $-4.25157292550^{\circ}$ \\
\hline $\mathrm{H}$ & -7.663585804633 \\
\hline $\mathrm{H}$ & -5.577087762492 \\
\hline $\mathrm{H}$ & -3.882573024269 \\
\hline $\mathrm{H}$ & -8.507056396905 \\
\hline $\mathrm{F}$ & -8.613806081547 \\
\hline $\mathrm{F}$ & -7.54879730392 \\
\hline $\mathrm{F}$ & -6.45145291140 \\
\hline $\mathrm{H}$ & -6.677268101429 \\
\hline $\mathrm{H}$ & -6.260324974949 \\
\hline $\mathrm{H}$ & -7.23319775475 \\
\hline $\mathrm{H}$ & -4.97888080172 \\
\hline & \\
\hline
\end{tabular}

4.881313635267

6.735166767889

6.844819751085

6.021486223694

3.472193134497

3.130868954050

0.129820911484

1.032920968647

3.577728404861

3. 026101167040

2.027845959801

2.588429589354

3.656807164537

4.091942998774

5.170307315014

4.703775061713

2.510283613040

0.779283149307

3.050097114723

4.933812887373

1.967989411306

1.783869593072

3.464378383368

3.603227296585

4.637198038786

6.025228386578

5.897303984963

5.186097983724

0.114203408409

3.749601386087

5.571487701583

4.006255350166

3. 266898270482

2.106778586033

2.600844355315

3.396912640415

4.237292752300

4.996165219353

5.154328750943

2.852895537336

0.943412003645

3.436134424527

4.366820354835

1.901935009130

1.764563949974

2.756598837473

3.688924470476

5.785784780836

4.135483663589

5.766209961070

5.539316433066

0.606108458779

4.039256318602

4.609079871655
$-2.153223368240$

$-2.347812541362$

$-0.494204615171$

$-0.515960689867$

$-1.511160860017$

0.187506396801

$-0.059465944309$

$-0.397477187166$

$-1.504473806622$

$-0.986429146962$

0.078956417428

0.574386850323

$-0.496918189577$

$-0.401592494685$

$-1.420080570246$

0.757598191821

$-1.810527180975$

$-0.571120858404$

1.892726638766

0.103390114438

0.921168933389

0.542372230844

$-0.977113926210$

$-0.123802702458$

$-2.606964812478$

$-1.619886577431$

$-1.000201029394$

0.519390536422

0.071077861789

1. 971805273455

$-0.553761753234$

$-0.378161619363$

$-0.870360679650$

0.128667840786

1.391725802147

0.815754432174

$-0.989981026895$

$-2.312586740952$

0.073872176781

$-1.859230325184$

$-0.266141334015$

2.192709397207

1. 667427642218

0.304038222079

2.011064499254

0.524476212138

$-0.968024957008$

$-2.437315370340$

$-3.348723591182$

$-2.425697081196$

0.103835891010

$-1.094832095790$

1.635739410414

2. 262743295366 


\begin{tabular}{|c|c|}
\hline & \\
\hline 0 & -2.210369395734 \\
\hline $\mathrm{C}$ & -3.466470499984 \\
\hline $\mathrm{C}$ & -3.369495987421 \\
\hline C & -2.436980657663 \\
\hline $\mathrm{C}$ & -1.108370980565 \\
\hline $\mathrm{C}$ & -1.353645365622 \\
\hline $\mathrm{C}$ & -4.356997773557 \\
\hline 0 & -2.778322579293 \\
\hline O & -2.268807303798 \\
\hline 0 & -0.355594121469 \\
\hline 0 & -0.126670069692 \\
\hline $\mathrm{H}$ & -3.945586517511 \\
\hline $\mathrm{H}$ & -4.365925003576 \\
\hline $\mathrm{H}$ & -2.894292548110 \\
\hline $\mathrm{H}$ & -0.585417810346 \\
\hline $\mathrm{H}$ & -1.822658745339 \\
\hline $\mathrm{F}$ & -4.467956566519 \\
\hline $\mathrm{F}$ & -5.591937512106 \\
\hline $\mathrm{F}$ & -3.905047187002 \\
\hline $\mathrm{H}$ & -3.290208030609 \\
\hline $\mathrm{H}$ & -2.043811268921 \\
\hline $\mathrm{H}$ & 0.496910824894 \\
\hline $\mathrm{H}$ & -0.229344900057 \\
\hline $5 b$ & 0804 \\
\hline 0 & -6.702499442277 \\
\hline $\mathrm{C}$ & -6.149680891260 \\
\hline $\mathrm{C}$ & -5.697738739913 \\
\hline $\mathrm{C}$ & -6.526776795889 \\
\hline $\mathrm{C}$ & -6.554149190226 \\
\hline $\mathrm{C}$ & -7.273887710940 \\
\hline $\mathrm{C}$ & -6.743914579448 \\
\hline 0 & -7.883977869052 \\
\hline $\mathrm{H}$ & -5.292358619944 \\
\hline 0 & -4.315090049038 \\
\hline 0 & -7.866580603027 \\
\hline 0 & -5.309342542745 \\
\hline $\mathrm{H}$ & -5.930105232273 \\
\hline $\mathrm{H}$ & -6.046297273058 \\
\hline $\mathrm{H}$ & -7.391523381862 \\
\hline $\mathrm{H}$ & -8.001586043038 \\
\hline $\mathrm{F}$ & -6.172554486825 \\
\hline $\mathrm{F}$ & -5.816590856628 \\
\hline $\mathrm{F}$ & -7.725822853209 \\
\hline $\mathrm{H}$ & -8.035061264470 \\
\hline $\mathrm{H}$ & -4.108254697885 \\
\hline $\mathrm{H}$ & -7.908780712980 \\
\hline $\mathrm{H}$ & -5.240426164012 \\
\hline $5 b$ & 0792 \\
\hline 0 & -5.838318208928 \\
\hline $\mathrm{C}$ & -6.042818608683 \\
\hline $\mathrm{C}$ & -5.451089529574 \\
\hline C & -5.437282397768 \\
\hline $\mathrm{C}$ & -5.115551290876 \\
\hline $\mathrm{C}$ & -7.557103088601 \\
\hline $\mathrm{C}$ & -7.899540277650 \\
\hline 0 & -8.055300661862 \\
\hline $\mathrm{H}$ & -5.507529223959 \\
\hline
\end{tabular}

5.910832490180

5.295029631697

3. 877140349913

3. 041507419668

3.776433121615

5.205818341827

6.218463587531

3.839449019335

1.760071632603

3.082473804725

5.845480519821

5.227319676339

3.415157912351

2. 902949770035

3.836246100712

5.186501603539

7.423829969355

5.692310939204

6.395553811641

4.349199506916

1.869217530655

3.526633817587

6.731379442749

4.446962135682

4.290087863200

2.816916372689

2.148665036859

3.256174139943

4.707722926408

4.998938050019

5.892890639676

4.962817409311

2.653907854647

1. 939022202378

3.225050758801

2.389698103914

1. 240453696292

3.189631264784

3.887930764125

3. 897455838725

5.964664941967

5.375507111050

5.803930132491

2.890065327273

1.191722131354

4.004785298410

5.254452412558

4.410253934599

3.033225991465

3.092426327695

4.561907667642

4.436287587611

3.947577433415

5. 742829432172

4.840692232596
$-0.149792912735$

$-0.235437760904$

$-0.815059850277$

0.071714860726

0.258913162872

0.736172175019

$-1.049789293011$

$-2.098217666861$

$-0.470653969944$

1.223095764674

0.765121491413

0.757295011462

$-0.823983803232$

1.059681835936

$-0.706766636562$

1.737679021350

$-0.488045477669$

$-1.145673652094$

$-2.304439542030$

$-2.736267768686$

$-1.405445888801$

1. 311560201919

1.133340697436

0.993838134428

$-0.330299040325$

$-0.430227727175$

0.683169131577

1.726233410003

$-1.290304991522$

$-2.686015886964$

$-0.860596045547$

$-0.437410040783$

$-0.235267029027$

0.289217915057

2.386560173468

$-1.408832527804$

1.061409937356

2. 426323818117

$-1.380826030264$

$-3.217440499234$

$-2.683736105766$

$-3.511065165434$

0.090341089182

0.681029351583

$-0.318045761799$

2. 951943890872

0.386836631606

$-0.765975999229$

$-0.394410583547$

1.144992392889

1.374015525415

$-1.023238904226$

$-2.422393824112$

$-0.944594627508$

$-1.618739296158$ 


\begin{tabular}{|c|c|}
\hline & \\
\hline 0 & -4.168208518909 \\
\hline 0 & -6.720678434957 \\
\hline 0 & -3.727966325745 \\
\hline $\mathrm{H}$ & -6.078812164440 \\
\hline $\mathrm{H}$ & -4.674191085373 \\
\hline $\mathrm{H}$ & -5.434637764795 \\
\hline $\mathrm{H}$ & -8.061262011605 \\
\hline $\mathrm{F}$ & -7.301592957374 \\
\hline $\mathrm{F}$ & -9.217224115663 \\
\hline $\mathrm{F}$ & -7.502301598512 \\
\hline $\mathrm{H}$ & -7.667040238834 \\
\hline $\mathrm{H}$ & -3.559494787663 \\
\hline $\mathrm{H}$ & -6.938701857922 \\
\hline $\mathrm{H}$ & -3.516354850306 \\
\hline $5 a_{-}$ & 0112 \\
\hline 0 & -5.011862915262 \\
\hline $\mathrm{C}$ & -6.163893948659 \\
\hline $\mathrm{C}$ & -5.741253156214 \\
\hline $\mathrm{C}$ & -4.210835656213 \\
\hline $\mathrm{C}$ & -3.997566314692 \\
\hline $\mathrm{C}$ & -7.296375344986 \\
\hline $\mathrm{C}$ & -8.633677225212 \\
\hline 0 & -7.439945034973 \\
\hline $\mathrm{H}$ & -6.422446954456 \\
\hline 0 & -6.166208325226 \\
\hline 0 & -3.548060117671 \\
\hline 0 & -4.157410340886 \\
\hline $\mathrm{H}$ & -6.074298010941 \\
\hline $\mathrm{H}$ & -3.863335802877 \\
\hline $\mathrm{H}$ & -3.040207564528 \\
\hline $\mathrm{H}$ & -7.107454765722 \\
\hline $\mathrm{F}$ & -8.599612162684 \\
\hline $\mathrm{F}$ & -8.939523623172 \\
\hline $\mathrm{F}$ & -9.631538097265 \\
\hline $\mathrm{H}$ & -6.549500016399 \\
\hline $\mathrm{H}$ & -7.086261591373 \\
\hline $\mathrm{H}$ & -3.665737211643 \\
\hline $\mathrm{H}$ & -3.914495818946 \\
\hline $5 a_{-}$ & 0399 \\
\hline 0 & -6.535792401499 \\
\hline $\mathrm{C}$ & -5.640314987970 \\
\hline $\mathrm{C}$ & -4.932818551231 \\
\hline $\mathrm{C}$ & -5.736945912130 \\
\hline $\mathrm{C}$ & -6.186639086564 \\
\hline $\mathrm{C}$ & -6.423166875225 \\
\hline $\mathrm{C}$ & -7.266661992198 \\
\hline 0 & -7.233597462026 \\
\hline $\mathrm{H}$ & -4.881690251308 \\
\hline 0 & -3.605622868626 \\
\hline 0 & -6.866652229138 \\
\hline 0 & -7.309403935548 \\
\hline $\mathrm{H}$ & -4.970958762680 \\
\hline $\mathrm{H}$ & -5.132342944351 \\
\hline $\mathrm{H}$ & -5.378280920626 \\
\hline $\mathrm{H}$ & -5.694301430709 \\
\hline $\mathrm{F}$ & -7.712352945736 \\
\hline $\mathrm{F}$ & -6.528962716936 \\
\hline
\end{tabular}

2.825919225943

2.875370953958

4.710891836772

2.214424244050

2.433833871365

4.942299990116

3.767509417839

4.676260965059

3.987386083894

2.668417640884

6.146032472944

3. 451814143864

1.938173256509

5.653012877049

4.923857703735

4.067174331487

2.876696405228

2. 962618742441

4.472566240753

4.933394783035

4.214444103496

6.114102956133

3.715808319300

1. 635303829021

2.363797490371

4.974423770988

3.022446328565

2.477646888799

4.778864193054

5.141308621482

3.081659559669

3. 845321818207

4.964807481029

6.430668128692

1. 490133336963

2.931623231460

5.906631736094

3.691341360568

4.046523529495

2.740854940190

1.657803809248

2.412041554036

4.700276531494

5.878110219398

3.821015436999

4.742967215373

2.804065160282

1.334456352703

1.788199014337

2.595126739061

0.771514201833

2.495633644260

5.137652907014

6.601025673263

6.703150498377
$-0.930772147055$

1. 692594658280

1.176989880017

$-0.754575272423$

1. 572478594828

2. 348312932123

$-0.310244325146$

$-3.373176677170$

$-2.643289284236$

$-2.590114245097$

$-0.156103799151$

$-0.511390940161$

1.636473833090

1.172325476971

$-1.308448725543$

$-1.188468294766$

$-0.307269603248$

$-0.342868093998$

$-0.444252719070$

$-0.632900419674$

$-0.713517953404$

$-1.371799938112$

$-2.193501005043$

$-0.806221151438$

0.729054940122

0.860910132080

0.730113184261

$-1.260751934246$

$-0.873409797929$

0.432048591440

0.034838407139

$-1.961981795329$

$-0.246147668955$

$-1.577642496869$

$-0.556433352694$

1.503340263502

0.893009431776

0.587818543806

$-0.465268598527$

$-0.900500440212$

$-0.174534523478$

1.075965519858

$-1.601353865697$

$-1.110107838325$

$-2.321649496948$

$-0.090162979076$

$-0.415033773748$

$-0.957065392783$

1.609499084145

$-1.985859992348$

0.051380123903

1.816676375970

$-2.296739573624$

$-2.149867897042$

$-0.339045319483$ 


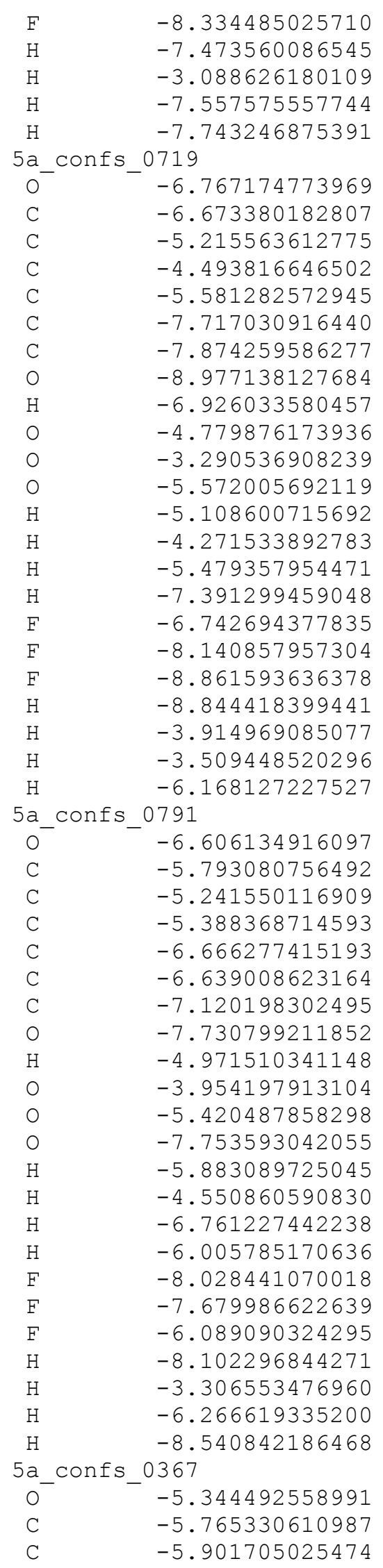

5.500074480543

3.058212583498

2.085335474072

1.023131973837

2. 387486700120

4.195803000722

4.553926785675

4. 325663242712

4.195774142744

3.561578261094

3.707411486634

4.126007081931

3.855498160912

5.615451803447

5.412314443751

3. 482666540243

2. 189654377157

3.378626982384

5.201575041597

3. 722580862864

2.655507145220

3.873081932610

5.431839397099

3.448167737509

3. 845455416144

5.204607313178

2.541358583876

1. 707450260496

3.515427193637

4.028882285761

2.805271564963

1. 685732262690

2.115567149782

4.923030046854

6.185219012476

4.242377499244

4.608918796720

2.998372215884

0.384824431248

1.684928039445

2. 578913819248

1.737912096614

1.738614163959

5.264338753079

5.923681815186

7.041002998140

6.825866173047

3.654668453960

3.016358615404

0.266201208111

1. 571891404547

4.803024602437

4.052113509913

2. 597011100661
$-0.402454349615$

$-1.769136298104$

$-0.795891292424$

$-0.352116175096$

2. 229448158845

1. 364448447711

$-0.030593099503$

$-0.439530746724$

0.910248255257

1.773459139402

$-0.762698405067$

$-2.217341775953$

$-0.167766492986$

$-0.122124131108$

$-1.217924503780$

0.864006029149

1. 457479974006

$-0.988305756404$

1.280565907398

2.849882699449

$-0.766854895164$

$-2.902834676484$

$-2.340955989068$

$-2.812975505138$

0.789756555325

$-1.590163933829$

0.810405123470

2.041817780041

0.658518637877

$-0.408035350840$

$-1.162582051763$

$-0.124090152031$

0.601321817598

$-1.311022576261$

$-0.598369122088$

$-1.856083082989$

0.029167806498

$-1.686961280313$

$-0.625068004610$

$-0.192726869228$

$-2.021196601574$

0.584213210623

1. 622424849458

$-2.139345199734$

0.350161319654

$-1.464525154759$

$-0.011434324036$

$-1.178907753689$

$-0.971685500161$

$-1.079357058630$

0.351582440995

0.568751307666

$-0.573688256253$

$-0.089631357010$ 


\begin{tabular}{|c|c|}
\hline & \\
\hline $\mathrm{C}$ & -6.200988000935 \\
\hline $\mathrm{C}$ & -5.342201818046 \\
\hline $\mathrm{C}$ & -7.078517911114 \\
\hline C & -7.244268746793 \\
\hline 0 & -7.068403795505 \\
\hline $\mathrm{H}$ & -4.982754458858 \\
\hline 0 & -4.671508623224 \\
\hline 0 & -7.569096331053 \\
\hline 0 & -5.932375943345 \\
\hline $\mathrm{H}$ & -6.712362685969 \\
\hline $\mathrm{H}$ & -5.908722023737 \\
\hline $\mathrm{H}$ & -4.307355402303 \\
\hline $\mathrm{H}$ & -7.935910866781 \\
\hline $\mathrm{F}$ & -7.202252351861 \\
\hline $\mathrm{F}$ & -6.268798846389 \\
\hline $\mathrm{F}$ & -8.411233919367 \\
\hline $\mathrm{H}$ & -6.609423254477 \\
\hline $\mathrm{H}$ & -4.451520940185 \\
\hline $\mathrm{H}$ & -7.685269223584 \\
\hline $\mathrm{H}$ & -5.361506661023 \\
\hline $5 b$ & 0928 \\
\hline 0 & -5.770201626398 \\
\hline $\mathrm{C}$ & -6.341481314959 \\
\hline $\mathrm{C}$ & -6.204321738938 \\
\hline $\mathrm{C}$ & -4.814534499422 \\
\hline $\mathrm{C}$ & -4.680573622696 \\
\hline $\mathrm{C}$ & -7.792766301090 \\
\hline $\mathrm{C}$ & -7.915756958439 \\
\hline 0 & -8.477071883314 \\
\hline $\mathrm{H}$ & -5.780586549372 \\
\hline 0 & -6.363313241298 \\
\hline 0 & -4.721665738717 \\
\hline 0 & -3.456070164630 \\
\hline $\mathrm{H}$ & -6.943762974862 \\
\hline $\mathrm{H}$ & -4.070522370955 \\
\hline $\mathrm{H}$ & -4.768961123976 \\
\hline $\mathrm{H}$ & -8.288906659982 \\
\hline $\mathrm{F}$ & -7.223201052783 \\
\hline $\mathrm{F}$ & -7.452901806541 \\
\hline $\mathrm{F}$ & -9.192026467237 \\
\hline $\mathrm{H}$ & -7.922130573386 \\
\hline $\mathrm{H}$ & -6.394434417050 \\
\hline $\mathrm{H}$ & -3.795917449681 \\
\hline $\mathrm{H}$ & -3.384891464276 \\
\hline & 0106 \\
\hline 0 & -2.288936807371 \\
\hline $\mathrm{C}$ & -3.430028660589 \\
\hline $\mathrm{C}$ & -3.615142270309 \\
\hline $\mathrm{C}$ & -2.331564485429 \\
\hline $\mathrm{C}$ & -1.130318124646 \\
\hline $\mathrm{C}$ & -1.078898608933 \\
\hline C & -4.602728528937 \\
\hline 0 & -3.86678856354 \\
\hline 0 & -2.466610601049 \\
\hline 0 & 0.064434404875 \\
\hline 0 & -0.78430765923 \\
\hline $\mathrm{H}$ & -3.36559399714 \\
\hline
\end{tabular}

2.773882956976

3.992541399569

4. 673840951144

4.380410263874

6.067127437426

4.143907441824

1. 905888450045

3.072652448581

4. 670173533298

2.059775163804

1.887609282094

3.712142111782

4.225884909170

3.044576926608

4.925887490207

4.826342220652

6.268811651397

1.741166737138

3.606604745251

5.387624666148

3.925681412015

3.732272355499

2.230532020569

1. 969963305265

3.028200120560

4.196754129729

5.718501983866

3.679080063760

4.274722722223

1.922159125467

0.635010592855

3.678635766500

1.708793325435

2.191815453697

2.594059143991

3.836844632048

6.253499186146

6.264542488748

6.100002197965

3.833703339141

0.964056981796

0.398615194469

4.388554458256

5.797236936804

4.984569674649

3.943659748877

3.119604555696

4.039331171067

5.091075396186

5.947218592292

4.592440801921

2.243035469593

3.288560988782

4.395885204743

4.456215460084
1.393390705514

1.738578403149

$-1.093991013924$

$-2.576098247507$

$-0.967531482227$

$-1.336520455640$

$-0.167593958737$

1. 529641802690

2. 802215748667

$-0.592527908835$

1. 967394509092

1. 967830588546

$-0.573103004820$

$-2.795107427468$

$-3.315062972830$

$-3.047009982561$

$-0.139506966538$

$-1.091419433992$

2. 328334673744

3.098654729274

0.172945707189

$-1.119141641086$

$-1.335069553959$

$-0.771421795880$

0.337346571359

$-1.083024158544$

$-1.094700955159$

0.021472331136

$-1.892759634417$

$-2.696019362977$

$-0.329377976450$

0.168298789224

$-0.709653410664$

$-1.548212594380$

1.340340716547

$-1.991371153884$

$-2.116594029588$

0.041040665815

$-1.231824468343$

0.800638891778

$-2.797625468522$

$-0.204658309864$

0.818370840670

0.180539080962

0.373151375574

$-0.728386078701$

$-0.809698246970$

$-1.009367555593$

0.095667461222

0.499704468056

$-1.949315507058$

$-1.908792071581$

$-1.072205557625$

1.284689997548

1.335065278032 


\begin{tabular}{|c|c|c|c|}
\hline $\mathrm{H}$ & -4.451774023674 & 3.286509784801 & -0.449898371798 \\
\hline $\mathrm{H}$ & -2.205397232079 & 2.552953662881 & 0.125841026267 \\
\hline $\mathrm{H}$ & -1.238026690940 & 4.553969738529 & -1.968378928779 \\
\hline $\mathrm{H}$ & -0.318639862726 & 5.848386978658 & -0.117774048006 \\
\hline $\mathrm{F}$ & -4.692660156891 & 6.810519640641 & -0.506419268188 \\
\hline $\mathrm{F}$ & -4.499737669711 & 6.658834328203 & 1.637194358479 \\
\hline $\mathrm{F}$ & -5.754614520619 & 5.254573390746 & 0.566395673833 \\
\hline $\mathrm{H}$ & -3.797940287913 & 3.922907203082 & -2.644441450174 \\
\hline $\mathrm{H}$ & -1.585948675682 & 1.909642227039 & -2.129833564035 \\
\hline $\mathrm{H}$ & 0.293813274661 & 3.029631775386 & -0.167939505428 \\
\hline $\mathrm{H}$ & -0.578290252111 & 5.025837269338 & 1.985701433962 \\
\hline \multicolumn{4}{|c|}{$6 a$ confs 0051} \\
\hline $0^{-}$ & -2.294928319876 & 5.891725889623 & 0.136271228761 \\
\hline $\mathrm{C}$ & -3.333299367842 & 4.953763604891 & 0.345906988114 \\
\hline $\mathrm{C}$ & -3.390064482349 & 3.954281198138 & -0.809489843653 \\
\hline $\mathrm{C}$ & -2.030706571251 & 3.257002007667 & -0.892301915755 \\
\hline $\mathrm{C}$ & -0.902296359535 & 4.284199923580 & -0.980472651031 \\
\hline $\mathrm{C}$ & -1.010376441538 & 5.315604988223 & 0.144108664942 \\
\hline $\mathrm{C}$ & -4.604433469948 & 5.775027768804 & 0.498506594191 \\
\hline 0 & -3.677802390487 & 4.560565280785 & -2.057977864519 \\
\hline 0 & -1.963953088988 & 2.383916386516 & -1.999126783826 \\
\hline 0 & 0.355444814850 & 3.656732906183 & -0.844911907182 \\
\hline O & -0.790375263881 & 4.713468606393 & 1.388475520073 \\
\hline $\mathrm{H}$ & -3.185825569824 & 4.415331039287 & 1.290516657056 \\
\hline $\mathrm{H}$ & -4.172778598542 & 3.210494505257 & -0.628422367116 \\
\hline $\mathrm{H}$ & -1.879028574288 & 2.643702261760 & 0.003697408662 \\
\hline $\mathrm{H}$ & -0.971395612304 & 4.814190788834 & -1.942263318662 \\
\hline $\mathrm{H}$ & -0.322123324445 & 6.151566750867 & -0.019953437792 \\
\hline $\mathrm{F}$ & -5.674358857101 & 4.965043787764 & 0.546928199547 \\
\hline $\mathrm{F}$ & -4.782102252195 & 6.628653212350 & -0.521350941875 \\
\hline $\mathrm{F}$ & -4.585508167460 & 6.494671015874 & 1.625052738716 \\
\hline $\mathrm{H}$ & -3.420589292911 & 5.492907109375 & -2.036703685216 \\
\hline $\mathrm{H}$ & -2.395887559567 & 2.833224953111 & -2.741610703415 \\
\hline $\mathrm{H}$ & 0.368532380236 & 2.889577586632 & -1.433967250295 \\
\hline $\mathrm{H}$ & -0.043843630754 & 4.104148428086 & 1.286488670275 \\
\hline \multicolumn{4}{|c|}{ 6a_confs_0023 } \\
\hline 0 & -2.299034948548 & 5.870566028808 & 0.196351234522 \\
\hline $\mathrm{C}$ & -3.371268151172 & 4.980798469784 & 0.390178953810 \\
\hline $\mathrm{C}$ & -3.476186527600 & 3.949095310628 & -0.742890427023 \\
\hline $\mathrm{C}$ & -2.150885076244 & 3.194342951358 & -0.792652334061 \\
\hline C & -0.990299314449 & 4.182324461525 & -0.918026880652 \\
\hline $\mathrm{C}$ & -1.045737830030 & 5.237298473059 & 0.189393286205 \\
\hline $\mathrm{C}$ & -4.620409777165 & 5.832384157155 & 0.531728704062 \\
\hline 0 & -3.618431637656 & 4.516998265072 & -2.030715058743 \\
\hline 0 & -2.109863522733 & 2.269313185236 & -1.858509489386 \\
\hline O & 0.244327454662 & 3.508043459496 & -0.773904384229 \\
\hline 0 & -0.833813920898 & 4.642346195642 & 1.442019780211 \\
\hline $\mathrm{H}$ & -3.262040235542 & 4.442674074723 & 1.342502207204 \\
\hline $\mathrm{H}$ & -4.296029609934 & 3.249951144381 & -0.527737263758 \\
\hline $\mathrm{H}$ & -2.026753626120 & 2.615899560631 & 0.129343899301 \\
\hline $\mathrm{H}$ & -1.055095237740 & 4.693572661101 & -1.88754833702 \\
\hline $\mathrm{H}$ & -0.321451234166 & 6.037187152955 & 0.00397061868 \\
\hline $\mathrm{F}$ & -4.894964725016 & 6.534525236587 & -0.587624197818 \\
\hline $\mathrm{F}$ & -4.525995381328 & 6.700484121648 & 1.536255873906 \\
\hline $\mathrm{F}$ & -5.684354653974 & 5.040591869459 & 0.763263361861 \\
\hline $\mathrm{H}$ & -4.384395158173 & 5.101146197834 & -2.073109601049 \\
\hline $\mathrm{H}$ & -2.486216455797 & 2.709571648863 & -2.63501931850 \\
\hline
\end{tabular}




\begin{tabular}{|c|c|}
\hline $\mathrm{H}$ & 0.224716859389 \\
\hline $\mathrm{H}$ & -0.111117289763 \\
\hline \multicolumn{2}{|c|}{$5 a$ confs 0182} \\
\hline $0^{-}$ & -5.002103834134 \\
\hline $\mathrm{C}$ & -6.184897404887 \\
\hline $\mathrm{C}$ & -5.895304162021 \\
\hline $\mathrm{C}$ & -4.365346096510 \\
\hline $\mathrm{C}$ & -4.033758718776 \\
\hline $\mathrm{C}$ & -7.373598425441 \\
\hline $\mathrm{C}$ & -7.724015654478 \\
\hline 0 & -7.174252819850 \\
\hline $\mathrm{H}$ & -6.355085936164 \\
\hline 0 & -6.488532841083 \\
\hline 0 & -3.818794189542 \\
\hline 0 & -4.158001959357 \\
\hline $\mathrm{H}$ & -6.274267817856 \\
\hline $\mathrm{H}$ & -4.002456098824 \\
\hline $\mathrm{H}$ & -3.049147092949 \\
\hline $\mathrm{H}$ & -8.253309307358 \\
\hline $\mathrm{F}$ & -6.792072911645 \\
\hline $\mathrm{F}$ & -8.885889227676 \\
\hline $\mathrm{F}$ & -7.859927223042 \\
\hline $\mathrm{H}$ & -6.252789190412 \\
\hline $\mathrm{H}$ & -6.345011300637 \\
\hline $\mathrm{H}$ & -3.903215856952 \\
\hline $\mathrm{H}$ & -3.640321930407 \\
\hline \multicolumn{2}{|c|}{ 5b confs 0112} \\
\hline 0 & -6.337198225977 \\
\hline $\mathrm{C}$ & -5.841938348861 \\
\hline $\mathrm{C}$ & -5.351797697167 \\
\hline $\mathrm{C}$ & -5.951212075069 \\
\hline $\mathrm{C}$ & -5.925289502963 \\
\hline C & -6.996353980988 \\
\hline $\mathrm{C}$ & -7.235656330003 \\
\hline 0 & -8.189300526411 \\
\hline $\mathrm{H}$ & -4.994019061446 \\
\hline 0 & -3.948414609658 \\
\hline 0 & -7.258536612007 \\
\hline 0 & -4.591272759599 \\
\hline $\mathrm{H}$ & -5.725400447906 \\
\hline $\mathrm{H}$ & -5.330637081596 \\
\hline $\mathrm{H}$ & -6.620002613275 \\
\hline $\mathrm{H}$ & -6.762075109320 \\
\hline $\mathrm{F}$ & -7.690974820355 \\
\hline $\mathrm{F}$ & -8.141834042234 \\
\hline $\mathrm{F}$ & -6.103465570348 \\
\hline $\mathrm{H}$ & -8.384280658946 \\
\hline $\mathrm{H}$ & -3.603235573717 \\
\hline $\mathrm{H}$ & -7.730208158517 \\
\hline $\mathrm{H}$ & -4.520696193639 \\
\hline \multicolumn{2}{|c|}{ 5b confs 1170} \\
\hline $0^{-}$ & -6.177298050136 \\
\hline C & -5.907444834317 \\
\hline $\mathrm{C}$ & -5.485151748741 \\
\hline $\mathrm{C}$ & -5.795282935732 \\
\hline $\mathrm{C}$ & -5.578778210697 \\
\hline $\mathrm{C}$ & -7.17195743947 \\
\hline
\end{tabular}

2.730275228758

4.005310145299

4.972056461829

4.156820049703

2. 925558692552

2. 897212047879

4.390373608159

4.958549812284

6.139945134585

5.407373418925

3.848815401615

1.796674479577

2.186281712012

4.850631463243

3.080884211616

2.434580951647

4.640424257208

4.303543608792

7.101547830044

6.696501351284

5.742536447412

5.698327801450

1.033938711951

2.741835536411

5.654087009821

3. 671240159341

3.936291593681

2. 574524451060

1.558477400299

2. 378122188782

4.556111362914

6.020949270396

3.836422375122

4. 628371123178

2.509353222738

1.165585538302

2.396349612361

2.391467430570

0.663128711935

2.032628538148

4.534882239419

6.151449830473

6.575378518522

6.735714264862

3.874234946755

2. 506267136171

1. 903587444679

3.001662640294

4.211177437767

3. 795053216325

2. 302683088741

1.943930660510

3.293106978415

4.012745472702
$-1.348295096696$

1.338424469187

$-1.174479673832$

$-1.231462535672$

$-0.370958449414$

$-0.331824090704$

$-0.344654631173$

$-0.708603131693$

$-1.610942566183$

0.600424170625

$-2.268476324487$

$-0.965658648300$

0.742876666432

0.987235548099

0.649662287859

$-1.255985366862$

$-0.747182763482$

$-0.711259644056$

$-1.580007016244$

$-1.240052640332$

$-2.890913747562$

0.689920923164

$-0.392614240118$

1.531456299925

1.111699574008

0.464541051816

$-0.856464258244$

$-1.423154120225$

$-0.434001807644$

0.854781207018

$-1.650730246118$

$-1.294433508956$

$-1.459813169236$

$-0.816996640680$

$-1.494290712028$

$-0.762156916664$

1. 306578260583

$-2.434959600854$

$-0.361171558913$

1.624989272612

$-2.719521344216$

$-0.037466417375$

$-2.108344663405$

$-1.397947003243$

$-0.508889357141$

$-0.589174208099$

$-1.178508363124$

2.055434104133

0.391841041403

$-0.954415688650$

$-0.871149942377$

0.597052395789

1.267537446114

$-1.778080336506$ 


\begin{tabular}{|c|c|c|c|}
\hline & & & \\
\hline $\mathrm{C}$ & -7.591126451828 & 5.482762706061 & -1.823555442244 \\
\hline O & -8.242387632963 & 3.231355611040 & -1.334728599657 \\
\hline $\mathrm{H}$ & -5.081326821011 & 4.380440124447 & -1.372652292620 \\
\hline 0 & -4.132330409131 & 2.100517369286 & -1.196660070052 \\
\hline 0 & -7.155730811040 & 1.630803212662 & 0.811332177952 \\
\hline 0 & -4.184679218215 & 3.488203076373 & 1.341214309858 \\
\hline $\mathrm{H}$ & -6.071069652524 & 1.686152045192 & -1.559168667240 \\
\hline $\mathrm{H}$ & -5.119811205758 & 1.168597161372 & 0.973348324868 \\
\hline $\mathrm{H}$ & -6.065894093218 & 3.398628492800 & 2.240384315059 \\
\hline $\mathrm{H}$ & -6.959430771509 & 3.733496138240 & -2.817011959557 \\
\hline $\mathrm{F}$ & -8.069548613462 & 5.919402992457 & -0.653793607915 \\
\hline $\mathrm{F}$ & -8.547696384926 & 5.674746215614 & -2.744184781609 \\
\hline $\mathrm{F}$ & -6.548653305763 & 6.267897022716 & -2.161588099943 \\
\hline $\mathrm{H}$ & -8.223147096800 & 3.205756063602 & -0.363879185962 \\
\hline $\mathrm{H}$ & -3.594675860630 & 2.562757413252 & -0.536878394790 \\
\hline $\mathrm{H}$ & -7.398023030632 & 0.857571962696 & 0.287933119543 \\
\hline $\mathrm{H}$ & -4.013555421494 & 4.388215537731 & 1.646103938536 \\
\hline & 0067 & & \\
\hline 0 & -2.296456601540 & 5.815168369639 & 0.106595435322 \\
\hline $\mathrm{C}$ & -3.362858503701 & 4.913300838322 & 0.282154615691 \\
\hline $\mathrm{C}$ & -3.436232630094 & 3.861229269152 & -0.834081268785 \\
\hline $\mathrm{C}$ & -2.087239817943 & 3.142092605996 & -0.886288140703 \\
\hline $\mathrm{C}$ & -0.959564887090 & 4.167760529956 & -1.013796341404 \\
\hline $\mathrm{C}$ & -1.029060130281 & 5.168908935594 & 0.134530112530 \\
\hline $\mathrm{C}$ & -4.624634220622 & 5.748895032759 & 0.399752418745 \\
\hline 0 & -3.610877054078 & 4.407638125927 & -2.127882105640 \\
\hline 0 & -2.031167713694 & 2.231565124628 & -1.964107762559 \\
\hline O & 0.298504739762 & 3.545076266294 & -1.004714091672 \\
\hline 0 & -0.838315749679 & 4.486756921437 & 1.335241196970 \\
\hline $\mathrm{H}$ & -3.267659525426 & 4.380737264799 & 1.239723356754 \\
\hline $\mathrm{H}$ & -4.232972448657 & 3.141001533921 & -0.602448577721 \\
\hline $\mathrm{H}$ & -1.936237389354 & 2.561260887543 & 0.029587357196 \\
\hline $\mathrm{H}$ & -1.113609369013 & 4.739347603861 & -1.940735845438 \\
\hline $\mathrm{H}$ & -0.299253395699 & 5.972081056159 & -0.001140046546 \\
\hline $\mathrm{F}$ & -4.557420759787 & 6.611844580096 & 1.415001395948 \\
\hline $\mathrm{F}$ & -5.684602182441 & 4.945298398194 & 0.609722842453 \\
\hline $\mathrm{F}$ & -4.887619601201 & 6.456141757798 & -0.716219049008 \\
\hline $\mathrm{H}$ & -4.420564767625 & 4.928043136163 & -2.181687547032 \\
\hline $\mathrm{H}$ & -2.428593418182 & 2.670636826603 & -2.730702189424 \\
\hline $\mathrm{H}$ & 0.246904633178 & 2.789116256373 & -1.605231945081 \\
\hline $\mathrm{H}$ & -0.637169206832 & 5.117398678790 & 2.036026179403 \\
\hline $6 \mathrm{~b}$ & 0059 & & \\
\hline 0 & -2.573305412181 & 5.517635453395 & 0.479005418150 \\
\hline $\mathrm{C}$ & -3.649374974477 & 5.114548701923 & -0.334398967034 \\
\hline $\mathrm{C}$ & -3.223645399738 & 4.179168144749 & -1.468867770668 \\
\hline $\mathrm{C}$ & -2.466107652520 & 2.999403785802 & -0.856923634099 \\
\hline C & -1.351153722945 & 3.501127992324 & 0.045210376780 \\
\hline $\mathrm{C}$ & -1.933588931942 & 4.422578317921 & 1.105838402637 \\
\hline $\mathrm{C}$ & -4.315519885780 & 6.394656405560 & -0.823228886897 \\
\hline 0 & -2.407089307327 & 4.880738685328 & -2.369618372307 \\
\hline 0 & -1.949697397561 & 2.239784285973 & -1.927960754822 \\
\hline 0 & -0.740425594691 & 2.361471433182 & 0.612940639577 \\
\hline 0 & -0.878258761126 & 4.895124982452 & 1.867354770708 \\
\hline $\mathrm{H}$ & -4.413934583337 & 4.592799529855 & 0.266177729896 \\
\hline $\mathrm{H}$ & -4.129465125065 & 3.803367079114 & -1.966740868103 \\
\hline $\mathrm{H}$ & -3.159513911507 & 2.391192374499 & -0.253467646560 \\
\hline $\mathrm{H}$ & -0.637597774315 & 4.091810436852 & -0 \\
\hline
\end{tabular}




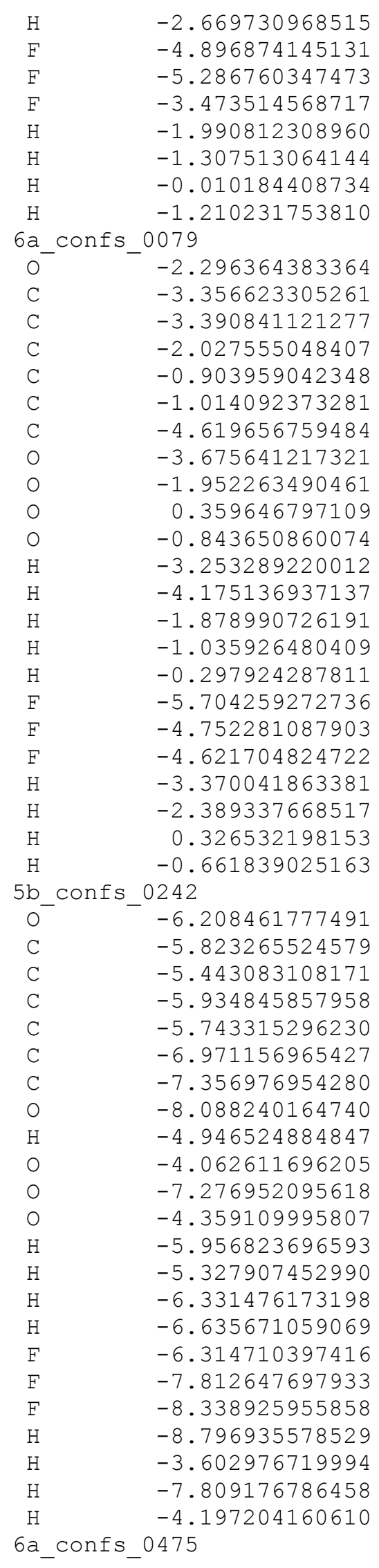

3.880263217270

7.035295677021

6.093860380234

7.244397946834

4.223638798436

1. 614307196087

2. 652528777702

5.508100397485

5.886265802331

4.963279814000

3.956791239511

3.258141795507

4.292386729590

5.263000398868

5.801957029187

4.556533721962

2.407104454780

3. 686218320689

4.558910664869

4.418040700020

3.213498159791

2. 633185389098

4.891741315685

6.083865825279

5.011794274166

6.667963961561

6.513723702040

5.474268810439

2.866557560390

2.944185218097

5.173885112140

3.853574119530

3. 909540791101

2.458746884496

1.629518413171

2. 640410231682

4. 482742124247

5.902079708075

3.636812072192

4.555754798629

2. 324134919260

1.238097414870

2.706022417218

2.143685579724

0.732373370946

2.436033486524

4.553495075409

6.736169506268

6.005521882736

6.341022942120

3. 917708363980

2. 448170197557

1. 998341902102

3.423043798162
1.728686796817

0.207437931083

$-1.704031064402$

$-1.399835160758$

$-2.945064931501$

$-1.565654175580$

1. 173688459088

2. 535109403091

0.172389370144

0.338755336775

$-0.811063975789$

$-0.878645339374$

$-0.943449914007$

0.227414630081

0.453609036018

$-2.063849871309$

$-2.002401606194$

$-0.931792394582$

1.414679934781

1.285856036750

$-0.635938795564$

0.009053290122

$-1.859503640955$

0.128534425544

0.466309794133

$-0.560811105345$

1. 589215336346

$-2.064395312004$

$-2.735700784140$

$-1.551174319147$

2. 134609867715

0.444921625524

$-0.930096936784$

$-1.345086797534$

$-0.140617600642$

0.986550150255

$-1.746582662400$

$-1.341486648780$

$-1.617060769116$

$-1.056178194982$

$-1.583731143407$

$-0.244106634803$

1.257165310801

$-2.256742335955$

$-0.005196918290$

1.884691975246

$-2.791387855908$

$-1.481320912703$

$-0.097038757580$

$-2.163370815370$

$-2.208903694233$

$-0.740288884705$

$-0.527102311069$

1. 882970812434 


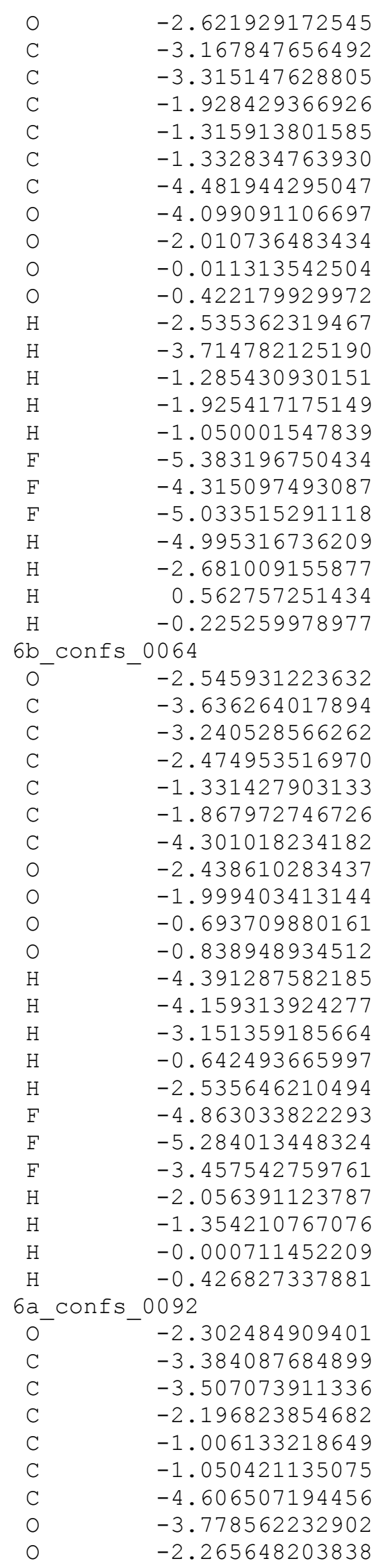

5.610783238342

5.432522215372

3. 949628802578

3.299297422663

3. 552853576373

5.050721604156

6.192151163191

3. 223336578188

1. 932828410403

3.050430509348

5.656843317475

5.910143701778

3.853857008252

3.750080457762

3.038799694456

5.222071904354

5.694251112055

7.483732352262

6.113321431688

3.574800589260

1.543053137780

3.562782540874

6.552709231389

5.511460524396

5.101559302226

4.149703005562

2. 975743150449

3.497121513331

4.436362704756

6.381033804023

4.835588924662

2.190102903537

2.374146600421

4.955960182180

4.594826477874

3.774731441109

2.386123160400

4.070354258899

3.891149534921

7.039434851540

6.076455273053

7.216073243061

4.169912186923

1.562703749359

2.683192776865

5.674860430453

5.804833199078

4.939497021802

3.862225977796

3.060823502450

4.012765165885

5.142349203615

5.835876701389

4.401065824762

2.098528922815
$-1.198746742149$

0.087119725127

0.445015256237

0.382256506013

$-1.004672717044$

$-1.329444834866$

0.108096979210

$-0.481206652308$

0.686762929242

$-1.128090270916$

$-0.445268026847$

0.849941647920

1.463900383794

1.147900568122

$-1.754094829880$

$-2.371779779950$

$-0.760328737551$

$-0.183809650499$

1.332835624396

$-0.530765577917$

0.108353867979

$-0.542887119512$

$-0.743088548600$

0.464629223048

$-0.328235429759$

$-1.459165863358$

$-0.849256391940$

0.002825658010

1.079170890026

$-0.822863883838$

$-2.386767413941$

$-1.920769932941$

0.571632870844

1. 854510338904

0.295352892439

$-1.932670800565$

$-0.209346013215$

$-0.636325645443$

1.764760455510

0.204873083199

$-1.688670858513$

$-1.421182774579$

$-2.975810427283$

$-1.567404573147$

1.169937651078

1.354276945467

0.062881900060

0.358548293604

$-0.713490072689$

$-0.768223472083$

$-0.959241083935$

0.075075036145

0.479046585841

$-1.996252360639$

$-1.786344043698$ 


\begin{tabular}{|c|c|}
\hline 0 & 0.236449448584 \\
\hline 0 & -0.793645008576 \\
\hline $\mathrm{H}$ & -3.246892492072 \\
\hline $\mathrm{H}$ & -4.331085754525 \\
\hline $\mathrm{H}$ & -2.068201493156 \\
\hline $\mathrm{H}$ & -1.078733266177 \\
\hline $\mathrm{H}$ & -0.315473824107 \\
\hline $\mathrm{F}$ & -4.512860133006 \\
\hline $\mathrm{F}$ & -5.715424386770 \\
\hline $\mathrm{F}$ & -4.768623584678 \\
\hline $\mathrm{H}$ & -3.441571217326 \\
\hline $\mathrm{H}$ & -2.607290643716 \\
\hline $\mathrm{H}$ & 0.363999129796 \\
\hline $\mathrm{H}$ & -0.581904429036 \\
\hline \multicolumn{2}{|c|}{$6 a$ confs 0563} \\
\hline 0 & -1.994507419492 \\
\hline C & -2.935091517168 \\
\hline $\mathrm{C}$ & -3.869181805065 \\
\hline $\mathrm{C}$ & -2.964209632158 \\
\hline $\mathrm{C}$ & -1.936004089058 \\
\hline $\mathrm{C}$ & -1.147297885072 \\
\hline $\mathrm{C}$ & -3.666074462863 \\
\hline 0 & -4.509591134633 \\
\hline 0 & -3.734518333424 \\
\hline 0 & -1.071435005624 \\
\hline 0 & -0.326870417551 \\
\hline $\mathrm{H}$ & -2.427965758877 \\
\hline $\mathrm{H}$ & -4.590000942345 \\
\hline $\mathrm{H}$ & -2.433223582206 \\
\hline $\mathrm{H}$ & -2.468692828852 \\
\hline $\mathrm{H}$ & -0.550188055341 \\
\hline $\mathrm{F}$ & -4.537782877076 \\
\hline $\mathrm{F}$ & -4.372084255072 \\
\hline $\mathrm{F}$ & -2.825355663007 \\
\hline $\mathrm{H}$ & -5.092492741213 \\
\hline $\mathrm{H}$ & -3.137013574393 \\
\hline $\mathrm{H}$ & -0.432755764131 \\
\hline $\mathrm{H}$ & 0.318337744622 \\
\hline \multicolumn{2}{|c|}{$5 a$ confs 0583} \\
\hline 0 & -5.505850348888 \\
\hline $\mathrm{C}$ & -6.661431165167 \\
\hline $\mathrm{C}$ & -6.156422780468 \\
\hline $\mathrm{C}$ & -4.714335727320 \\
\hline $\mathrm{C}$ & -4.337032871381 \\
\hline $\mathrm{C}$ & -7.509592103846 \\
\hline $\mathrm{C}$ & -8.128463637269 \\
\hline 0 & -6.698238442014 \\
\hline $\mathrm{H}$ & -7.256904147001 \\
\hline 0 & -6.994658762698 \\
\hline 0 & -3.866421460877 \\
\hline 0 & -3.940729411291 \\
\hline $\mathrm{H}$ & -6.134593638255 \\
\hline $\mathrm{H}$ & -4.715947095099 \\
\hline $\mathrm{H}$ & -3.530618399785 \\
\hline $\mathrm{H}$ & -8.351905177109 \\
\hline $\mathrm{F}$ & -8.832928438437 \\
\hline $\mathrm{F}$ & -8.98340660374 \\
\hline
\end{tabular}

3.364189943565

4.554885063580

4.462093597928

3.182100127782

2. 513815648367

4.464527459190

5.916701410037

6.631634681662

5.093866265661

6.626178768340

5.306128550282

2.539683381501

3.023586413283

5.236543169230

5.256887361970

5.488367834275

4.280088719362

3.125022257879

2.879800413519

4.158477846562

6.765368630030

3.896567705728

1. 977383544266

1.819330260310

4.378763875796

5.704102410575

4.491736327245

3.427534129478

2. 576898691075

4.053160864101

7.087750854828

6.650294396149

7.792433321931

4.598036052019

1.217791637759

2.162758446076

5.067444419068

4.061110122097

3.400783782292

2.233875567504

2.063951528124

3. 511446517629

4. 374944031180

5.479721222137

4.941905467079

3.009377783333

1.119614613381

1.454621524550

4.179169727880

2. 518785275707

1. 452946995109

3. 611591490208

3. 812332598734

6.311297200911

4.965356400907
$-0.914866084560$

1. 322179715859

1. 337566318527

$-0.474925266219$

0.174680991685

$-1.955507251427$

$-0.162608644873$

1. 554173661471

0.617367606176

$-0.590604674997$

$-2.048873874664$

$-2.577556176217$

$-0.019235839481$

1.970908736115

$-1.016594779592$

0.010249469582

0.202629669747

0.622661170366

$-0.482417171845$

$-0.774315508412$

$-0.361161060868$

$-0.988516671985$

0.858619186395

$-0.132192359020$

0.349028326895

0.961905839755

1.004785499859

1.541146651087

$-1.387321571671$

$-1.684679014592$

0.611700317125

$-1.500478278944$

$-0.513193035452$

$-1.299916147674$

0.826810072783

0.509039342247

0.149210054213

$-1.503475785857$

$-0.987580359742$

$-0.131091387156$

$-0.615581606438$

$-0.948808705811$

$-0.178793916557$

$-1.026402084469$

0.824437732108

$-1.820353524848$

$-0.328990061575$

0.318337745057

0.226446879004

0.931075015161

$-1.525565057497$

$-1.677743255249$

0.250027534196

$-0.223183250761$

$-1.922682276246$ 


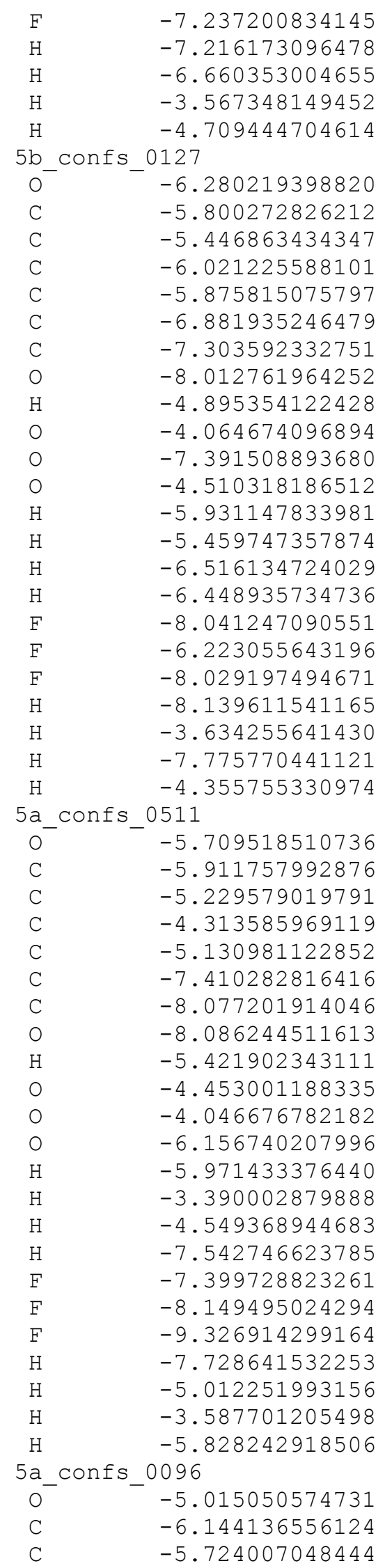

6.222212854989

5.539876818560

0.387872005094

2.153549750752

4.665656721844

3.749584491630

3.923149133932

2. 503426257761

1.589182116393

2.490258568236

4.616800075347

5.951424097092

3.829843152148

4.540592449972

2. 320827429357

1. 379373605810

2. 474839512615

2.291559145820

0.653369921174

2.230291987036

4.870759125495

6.661163323244

6.698677416595

5.805957497318

3.214115398410

2.410745864265

0.770879035425

3.149080394925

4.655180200981

4.010279136330

2.633253793778

2.816118406567

3.770030870154

3.895838005028

5.262229287290

3.142930948551

4.607067875611

2.271147582445

1.633895466709

3.071147483427

1.859153100268

3.312833797906

4. 363261573066

3. 397202373328

6.021766877821

5.938998469037

5.127226234997

3. 348449744100

1.872084864377

1.010637889629

2.188266018602

5.049958478582

4.171270784926

3.022195860026
$-1.677229184039$

1. 377749982504

0.204000713982

0.920403733536

0.568001120697

0.434804481006

$-0.908373018750$

$-1.431471009284$

$-0.335311702781$

0.889179047200

$-1.729257106008$

$-1.111609082571$

$-1.954548330259$

$-0.900325148063$

$-1.611922245363$

$-0.620443604311$

1. 240616933457

$-2.387257157147$

$-0.259089207018$

1.739327268643

$-2.705176002094$

$-1.979136586567$

$-0.804378096008$

0.001171759636

$-1.215114430056$

$-0.749214408525$

0.021187097779

1.914640547088

0.244053286731

$-1.020212436789$

$-0.911873298130$

0.293476192804

1.159568644379

$-1.301387108809$

$-1.460458878419$

$-0.341499272420$

$-1.797112564288$

$-2.034666429275$

1.009364550137

1.829538272911

$-0.676985879089$

$-0.033103134126$

1.869814605738

$-2.271526976506$

$-2.346154602386$

$-0.311853137291$

$-1.929647312880$

0.541096720858

$-2.709733509324$

0.433647372373

2.058654893802

$-1.362526385903$

$-1.273857985322$

$-0.352325316494$ 


\begin{tabular}{|c|c|}
\hline & \\
\hline $\mathrm{C}$ & -4.201585880158 \\
\hline $\mathrm{C}$ & -4.010964302748 \\
\hline $\mathrm{C}$ & -7.351081282420 \\
\hline C & -7.830935904369 \\
\hline 0 & -7.115060347632 \\
\hline $\mathrm{H}$ & -6.362093764753 \\
\hline 0 & -6.212731357835 \\
\hline 0 & -3.697315964925 \\
\hline 0 & -4.205180160373 \\
\hline $\mathrm{H}$ & -6.092885421672 \\
\hline $\mathrm{H}$ & -3.791991272116 \\
\hline $\mathrm{H}$ & -3.057590338521 \\
\hline $\mathrm{H}$ & -8.185151182890 \\
\hline $\mathrm{F}$ & -8.002053691286 \\
\hline $\mathrm{F}$ & -6.974722701624 \\
\hline $\mathrm{F}$ & -9.012818357622 \\
\hline $\mathrm{H}$ & -6.217465687148 \\
\hline $\mathrm{H}$ & -6.130589738422 \\
\hline $\mathrm{H}$ & -2.772730896052 \\
\hline $\mathrm{H}$ & -4.043957568135 \\
\hline & 0097 \\
\hline 0 & -2.290315389619 \\
\hline $\mathrm{C}$ & -3.381640427003 \\
\hline $\mathrm{C}$ & -3.490728689961 \\
\hline $\mathrm{C}$ & -2.190828819494 \\
\hline $\mathrm{C}$ & -0.995847972493 \\
\hline $\mathrm{C}$ & -1.033931530491 \\
\hline C & -4.598730572210 \\
\hline 0 & -3.699590260604 \\
\hline 0 & -2.220453354675 \\
\hline 0 & 0.220840688640 \\
\hline 0 & -0.750092018733 \\
\hline $\mathrm{H}$ & -3.258667799367 \\
\hline $\mathrm{H}$ & -4.336248012822 \\
\hline $\mathrm{H}$ & -2.117707719074 \\
\hline $\mathrm{H}$ & -1.047842665312 \\
\hline $\mathrm{H}$ & -0.311733186159 \\
\hline $\mathrm{F}$ & -4.739253094131 \\
\hline $\mathrm{F}$ & -4.515163099828 \\
\hline F & -5.714264183753 \\
\hline $\mathrm{H}$ & -3.335659956108 \\
\hline $\mathrm{H}$ & -1.319426005837 \\
\hline $\mathrm{H}$ & 0.395634784266 \\
\hline $\mathrm{H}$ & -0.580050715231 \\
\hline & 0025 \\
\hline 0 & -2.206885906584 \\
\hline $\mathrm{C}$ & -3.265445529409 \\
\hline $\mathrm{C}$ & -3.350442259051 \\
\hline $\mathrm{C}$ & -2.023747895708 \\
\hline $\mathrm{C}$ & -0.869127573294 \\
\hline $\mathrm{C}$ & -0.944389968983 \\
\hline $\mathrm{C}$ & -4.525742818616 \\
\hline 0 & -3.469451793244 \\
\hline 0 & -1.867579242930 \\
\hline 0 & 0.370939451698 \\
\hline 0 & -0.715475769097 \\
\hline $\mathrm{H}$ & -3.14633867035 \\
\hline
\end{tabular}

3.139985524197

4.656566774568

4.933120049919

6.019913640719

5.483943334318

3.777883244400

1.805706688834

2. 610594573615

5.286240968536

3.199074864395

2. 626138743107

4.975775469398

4.225884726656

5.521506485608

7.043341264270

6.516441684761

5.859836138524

1.124882869669

2.358456875548

4.639480955425

5.801997582924

4.915250018809

3. 945913055195

3.134161589838

4.076000418368

5.156135992671

5.807185823561

4.623757013900

2. 207189322885

3. 362082561974

4. 512882270708

4.356121676372

3.264964238268

2. 614566744018

4.556651307257

5.951421736628

6.703330252584

6.489392400781

5.064190339092

5.517217634585

1.881020771064

3.124401760046

5.169165488471

5.838004723206

4.924104366202

3.964118993213

3.203086644796

4.194026132365

5.224392903528

5.747431250120

4.611992232764

2.334367298911

3.529302395016

4.608054783647

4.335750588885
$-0.337644023591$

$-0.470002348018$

$-0.728424363586$

$-1.689207611593$

0.532385967283

$-2.272256249466$

$-0.864693170343$

0.873026650521

0.774344338427

0.667251736411

$-1.215453926952$

$-0.901524541998$

$-0.637479116219$

$-2.930333590651$

$-1.775911178063$

$-1.294473725309$

0.553515677461

$-0.187298988338$

0.771267105769

1.478621045972

0.121131586777

0.323848943755

$-0.855635298339$

$-0.856715950970$

$-0.994210284895$

0.088932457569

0.511489542666

$-2.071071280242$

$-1.908134286735$

$-0.961287534876$

1.303975739784

1.260130996014

$-0.713111268438$

0.114832020633

$-1.976654778616$

$-0.118342230278$

$-0.472462860751$

1.664497954965

0.560131685159

$-2.013368114712$

$-2.036076276064$

$-0.039298811944$

1.990498049538

0.162036769398

0.309346857274

$-0.891684085416$

$-0.905880345816$

$-0.999958738099$

0.130887739817

0.504477978356

$-2.133206887145$

$-2.007673572878$

$-0.848257349028$

1. 370417798764

1.230242575588 


\begin{tabular}{|c|c|c|c|}
\hline $\mathrm{H}$ & -4.184032906416 & 3.262464624661 & -0.723968777799 \\
\hline $\mathrm{H}$ & -1.935557913583 & 2.645055459615 & 0.038759634695 \\
\hline $\mathrm{H}$ & -0.932240444977 & 4.712077099055 & -1.963844839827 \\
\hline $\mathrm{H}$ & -0.233966037261 & 6.039184394808 & -0.041798762373 \\
\hline $\mathrm{F}$ & -4.813554323241 & 6.514092935085 & -0.567898371713 \\
\hline $\mathrm{F}$ & -4.440611642939 & 6.553643535362 & 1.560551224869 \\
\hline F & -5.577935599407 & 4.927920469306 & 0.690461957673 \\
\hline $\mathrm{H}$ & -4.190386855620 & 5.252190261029 & -2.115270456059 \\
\hline $\mathrm{H}$ & -2.629552063015 & 1.750177845770 & -2.089491727215 \\
\hline $\mathrm{H}$ & 0.396442505463 & 2.801467911728 & -1.483465734123 \\
\hline $\mathrm{H}$ & 0.010883256569 & 3.978593150927 & 1.247617111057 \\
\hline \multicolumn{4}{|c|}{ 6a_confs_0146 } \\
\hline 0 & -2.301801650946 & 5.846481806373 & 0.230303337813 \\
\hline C & -3.405539231526 & 4.974615416136 & 0.375952023042 \\
\hline C & -3.535253338854 & 3.972844314840 & -0.769815814576 \\
\hline C & -2.203900554759 & 3.235118697894 & -0.913575850062 \\
\hline C & -1.073695149782 & 4.243720297197 & -1.061522594844 \\
\hline C & -1.059618421190 & 5.179526993373 & 0.149174322902 \\
\hline C & -4.623694804185 & 5.874251815552 & 0.531565273682 \\
\hline 0 & -3.853009195539 & 4.656945118593 & -1.956784395636 \\
\hline 0 & -2.304933021527 & 2.430420546067 & -2.070482745127 \\
\hline 0 & 0.115050877654 & 3.494305623734 & -1.212834202913 \\
\hline 0 & -0.796885935210 & 4.421496763901 & 1.291462974625 \\
\hline $\mathrm{H}$ & -3.324164243574 & 4.408208772502 & 1.314277176416 \\
\hline $\mathrm{H}$ & -4.321251934285 & 3.248717985882 & -0.511897067374 \\
\hline $\mathrm{H}$ & -2.017875798309 & 2.619611132734 & -0.022687570292 \\
\hline $\mathrm{H}$ & -1.273777898657 & 4.859038336814 & -1.948177073771 \\
\hline $\mathrm{H}$ & -0.324530450664 & 5.983416952210 & 0.021540447707 \\
\hline $\mathrm{F}$ & -4.741951998620 & 6.782132564068 & -0.432048676118 \\
\hline $\mathrm{F}$ & -4.567509038972 & 6.533908348892 & 1.703649308216 \\
\hline $\mathrm{F}$ & -5.743500431077 & 5.128195412283 & 0.548606942439 \\
\hline $\mathrm{H}$ & -3.746305742091 & 4.025905366329 & -2.682347500107 \\
\hline $\mathrm{H}$ & -1.417885468004 & 2.106050174333 & -2.276620673622 \\
\hline $\mathrm{H}$ & 0.818938224207 & 4.050757831484 & -1.562887503640 \\
\hline $\mathrm{H}$ & -0.673504794092 & 5.015029728807 & 2.042049861240 \\
\hline \multicolumn{4}{|c|}{ 6a_confs_0093 } \\
\hline 0 & -2.346956513002 & 5.813352174064 & 0.203141423547 \\
\hline C & -3.487527313516 & 4.994817441003 & 0.358843288611 \\
\hline C & -3.628198478639 & 3.962520527964 & -0.758695991068 \\
\hline C & -2.337230417773 & 3.149157994658 & -0.816858273967 \\
\hline C & -1.143673178156 & 4.083049280310 & -0.956276426580 \\
\hline C & -1.139867800522 & 5.112363039653 & 0.181038347733 \\
\hline $\mathrm{C}$ & -4.672229987905 & 5.947985183365 & 0.443313288145 \\
\hline 0 & -3.849987168764 & 4.622744259021 & -1.980765372256 \\
\hline 0 & -2.438828700847 & 2.289832943970 & -1.933479568423 \\
\hline 0 & 0.019295447663 & 3.262197734510 & -0.888718804262 \\
\hline 0 & -0.950505717319 & 4.472895833575 & 1.417186171433 \\
\hline $\mathrm{H}$ & -3.448015970786 & 4.466373890163 & 1.321028704164 \\
\hline $\mathrm{H}$ & -4.464032388444 & 3.291657852005 & -0.514775996866 \\
\hline $\mathrm{H}$ & -2.231877147407 & 2.569079997635 & 0.111159178076 \\
\hline $\mathrm{H}$ & -1.211914463791 & 4.606834846943 & -1.916463483591 \\
\hline $\mathrm{H}$ & -0.370712521730 & 5.877751565849 & 0.016375114903 \\
\hline $\mathrm{F}$ & -4.628892546916 & 6.650443887223 & 1.587057170832 \\
\hline $\mathrm{F}$ & -5.820198410035 & 5.243034091477 & 0.450272944514 \\
\hline $\mathrm{F}$ & -4.726188179635 & 6.818416460790 & -0.560779273374 \\
\hline $\mathrm{H}$ & -3.793900057839 & 3.956911226390 & -2.679948738456 \\
\hline $\mathrm{H}$ & -1.588183579382 & 1.846953104634 & -2.04842916909 \\
\hline
\end{tabular}




\begin{tabular}{|c|c|}
\hline $\mathrm{H}$ & 0.755682786611 \\
\hline $\mathrm{H}$ & -0.287157691865 \\
\hline \multicolumn{2}{|c|}{ 6a confs 0132} \\
\hline 0 & -2.206941250676 \\
\hline $\mathrm{C}$ & -3.265586915411 \\
\hline $\mathrm{C}$ & -3.350557274080 \\
\hline $\mathrm{C}$ & -2.0239449950 \\
\hline $\mathrm{C}$ & -0.869233168358 \\
\hline $\mathrm{C}$ & -0.944492277450 \\
\hline $\mathrm{C}$ & -4.525824948590 \\
\hline 0 & -3.469147511116 \\
\hline 0 & -1.867806410947 \\
\hline 0 & 0.370758237318 \\
\hline 0 & -0.71555922499 \\
\hline $\mathrm{H}$ & -3.146575511396 \\
\hline $\mathrm{H}$ & -4.184301890622 \\
\hline $\mathrm{H}$ & -1.935832626799 \\
\hline $\mathrm{H}$ & -0.932244704871 \\
\hline $\mathrm{H}$ & -0.234027142068 \\
\hline $\mathrm{F}$ & -4.813550 \\
\hline $\mathrm{F}$ & -4.440657983752 \\
\hline $\mathrm{F}$ & -5.57808375958 \\
\hline $\mathrm{H}$ & -4.190049581035 \\
\hline $\mathrm{H}$ & -2.62749633997 \\
\hline $\mathrm{H}$ & 0.396071 \\
\hline $\mathrm{H}$ & 0.01078436389 \\
\hline \multicolumn{2}{|c|}{ 6b_confs_0053 } \\
\hline 0 & -2.39502011112 \\
\hline $\mathrm{C}$ & -3.52837767246 \\
\hline $\mathrm{C}$ & -3.095213671860 \\
\hline $\mathrm{C}$ & -2.2836866180 \\
\hline $\mathrm{C}$ & -1.14771615907 \\
\hline $\mathrm{C}$ & -1.66841604724 \\
\hline $\mathrm{C}$ & -4.32620157171 \\
\hline 0 & -2.31369773465 \\
\hline 0 & -1.75366659719 \\
\hline 0 & -0.55203471720 \\
\hline 0 & -0.59798556868 \\
\hline $\mathrm{H}$ & -4.18626615186 \\
\hline $\mathrm{H}$ & -3.96824021585 \\
\hline $\mathrm{H}$ & -2.93890460301 \\
\hline $\mathrm{H}$ & -0.40719984556 \\
\hline $\mathrm{H}$ & -2.35288358293 \\
\hline $\mathrm{F}$ & -3.58521408827 \\
\hline $\mathrm{F}$ & -4.83325182166 \\
\hline $\mathrm{F}$ & -5.35402300198 \\
\hline $\mathrm{H}$ & -1.80891924477 \\
\hline $\mathrm{H}$ & -1.43920094510 \\
\hline $\mathrm{H}$ & -0.34874494474 \\
\hline $\mathrm{H}$ & -0.91643508497 \\
\hline \multicolumn{2}{|c|}{$6 a$ confs 0105} \\
\hline 0 & -2.27805314720 \\
\hline $\mathrm{C}$ & -3.37659205654 \\
\hline $\mathrm{C}$ & -3.52914507504 \\
\hline $\mathrm{C}$ & -2.20647869325 \\
\hline $\mathrm{C}$ & -1.05131473278 \\
\hline 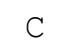 & -1.04603751758 \\
\hline
\end{tabular}

3.691587993547

3.778638671250

5.837984031835

4. 924123723317

3.964534117387

3.203308513477

4.194151137286

5.224317997876

5.747495029962

4. 612918017395

2.334736621672

3. 529288388281

4.607689795227

4.335499180507

3.262967141433

2.645225230239

4.712367434901

6.039109317250

6.514474549095

6.553425683988

4.928025504429

5.253146796528

1.747139046822

2.801429161783

3. 978243579311

5.558281690938

5.032869538089

4.066015002842

2.950194719444

3.510641294227

4.566341098690

6.227327063959

4.695504613241

2.066084266567

2.480080954783

5.188148274513

4.502901678545

3.639452640956

2. 350786323829

4.009430434322

4.109539387880

7.065270289660

6.927261388782

5.809630403425

5.419559381886

2.607756439610

1.761856052423

5.799767061387

5.820988123707

4.961207789862

3.934056296081

3.173606637419

4.147443809252

5.166999357020
$-1.338497137744$

1. 294572603726

0.162296665243

0.309298124537

$-0.892057533681$

$-0.906198698085$

$-1.000098682556$

0.130956024116

0.504621551734

$-2.133324686648$

$-2.008099523207$

$-0.848430402369$

1.370349947667

1.230035846581

$-0.724766470396$

0.038417677669

$-1.963902481626$

$-0.041556034838$

$-0.567562848921$

1.560908306350

0.690362920041

$-2.115326630035$

$-2.086779700063$

$-1.483620018648$

1.247376647134

0.393494456968

$-0.254446243211$

$-1.356451995284$

$-0.695419444584$

0.165849836614

1. 129789400645

$-0.758283179357$

$-2.353795716219$

$-1.657595698281$

0.911507408864

1. 728026006962

0.453585526658

$-1.860287411819$

$-0.049197091905$

$-0.481087262471$

1.866291474663

$-1.491159591349$

0.266170171874

$-1.514117838548$

$-1.955427086105$

$-2.397552165150$

0.296973121771

2. 402933319263

0.152599655069

0.355279187188

$-0.767384089623$

$-0.886865883450$

$-1.066710626993$

0.075146936178 


\begin{tabular}{|c|c|c|c|}
\hline & & & \\
\hline $\mathrm{C}$ & -4.594013327913 & 5.863589988861 & 0.505878120273 \\
\hline O & -3.830289683672 & 4.593607206510 & -1.971129824120 \\
\hline 0 & -2.207427419319 & 2.337411992857 & -2.039881441048 \\
\hline 0 & 0.189956365068 & 3.472403564553 & -1.011463360964 \\
\hline 0 & -0.792648295901 & 4.522733555152 & 1.298430088032 \\
\hline $\mathrm{H}$ & -3.272905797334 & 4.424671665450 & 1.308627380581 \\
\hline $\mathrm{H}$ & -4.334465408365 & 3.235731918578 & -0.489036945825 \\
\hline $\mathrm{H}$ & -2.031173527313 & 2.583812268781 & 0.022323951521 \\
\hline $\mathrm{H}$ & -1.174696177807 & 4.684349208570 & -2.015497848130 \\
\hline $\mathrm{H}$ & -0.308559358014 & 5.953367678908 & -0.116639107887 \\
\hline $\mathrm{F}$ & -4.525120951505 & 6.559956783268 & 1.651121305618 \\
\hline $\mathrm{F}$ & -5.709693288368 & 5.108223736284 & 0.563672799506 \\
\hline $\mathrm{F}$ & -4.736104740871 & 6.734989269706 & -0.488196929168 \\
\hline $\mathrm{H}$ & -3.692873663076 & 3.964125583133 & -2.692284642410 \\
\hline $\mathrm{H}$ & -2.624488659188 & 1.492597236932 & -1.836170076204 \\
\hline $\mathrm{H}$ & 0.179913642818 & 2.772088731504 & -1.676564551206 \\
\hline $\mathrm{H}$ & -0.036588486829 & 3.933037597612 & 1.163945903061 \\
\hline & 0601 & & \\
\hline 0 & -6.923236379654 & 3.771638554977 & 0.696115103377 \\
\hline $\mathrm{C}$ & -5.849179181266 & 4.045243474926 & -0.209633434719 \\
\hline $\mathrm{C}$ & -5.178439938362 & 2.690677026587 & -0.501230868239 \\
\hline $\mathrm{C}$ & -5.712766495139 & 1.802430776465 & 0.624427106772 \\
\hline $\mathrm{C}$ & -7.106494176189 & 2.390160612603 & 0.843151726109 \\
\hline $\mathrm{C}$ & -6.383887182532 & 4.706822471736 & -1.480492468245 \\
\hline $\mathrm{C}$ & -6.982540416391 & 6.085299736421 & -1.202736333286 \\
\hline 0 & -7.308739444679 & 3.911791100099 & -2.155890700425 \\
\hline $\mathrm{H}$ & -5.147159631895 & 4.724988589219 & 0.289672519494 \\
\hline 0 & -3.774652297052 & 2.727341263500 & -0.605052430566 \\
\hline 0 & -5.815591265670 & 0.439213915263 & 0.292923105327 \\
\hline 0 & -7.994496844149 & 1.935361017266 & -0.149470836996 \\
\hline $\mathrm{H}$ & -5.535948404182 & 2.296037909286 & -1.457992313005 \\
\hline $\mathrm{H}$ & -5.112685077231 & 1.949768484594 & 1.535700281197 \\
\hline $\mathrm{H}$ & -7.528909660093 & 2.222634720448 & 1.837308352535 \\
\hline $\mathrm{H}$ & -5.534114096733 & 4.881313635267 & -2.153223368240 \\
\hline $\mathrm{F}$ & -7.234042937520 & 6.735166767889 & -2.347812541362 \\
\hline $\mathrm{F}$ & -6.120012754031 & 6.844819751085 & -0.494204615171 \\
\hline $\mathrm{F}$ & -8.126800429586 & 6.021486223694 & -0.515960689867 \\
\hline $\mathrm{H}$ & -7.891175104679 & 3.472193134497 & -1.511160860017 \\
\hline $\mathrm{H}$ & -3.398563086326 & 3.130868954050 & 0.187506396801 \\
\hline $\mathrm{H}$ & -4.971839833493 & 0.129820911484 & -0.059465944309 \\
\hline $\mathrm{H}$ & -7.740725363147 & 1.032920968647 & -0.397477187166 \\
\hline $5 a_{-}$ & 0081 & & \\
\hline 0 & -4.955709573022 & 5.076191152621 & -1.154400029860 \\
\hline $\mathrm{C}$ & -6.135622016050 & 4.265204916448 & -1.225370005745 \\
\hline $\mathrm{C}$ & -5.852686804246 & 3.022797901562 & -0.378732431591 \\
\hline $\mathrm{C}$ & -4.324798580062 & 2.985055531260 & -0.35813361350 \\
\hline C & -3.999446622826 & 4.481419575924 & -0.305880111214 \\
\hline $\mathrm{C}$ & -7.317573754194 & 5.076109566690 & -0.680384153934 \\
\hline $\mathrm{C}$ & -7.881215285927 & 6.053002038557 & -1.705536742111 \\
\hline 0 & -6.944928260437 & 5.775489951261 & 0.481351062777 \\
\hline $\mathrm{H}$ & -6.309742669224 & 3.962961454716 & -2.263584005042 \\
\hline 0 & -6.478794491405 & 1.909452460054 & -0.963882180625 \\
\hline 0 & -3.777964055375 & 2.239663311856 & 0.694357903268 \\
\hline 0 & -4.079364880160 & 4.957909577168 & 1.016408679640 \\
\hline $\mathrm{H}$ & -6.216591906464 & 3.169545655049 & 0.652178257626 \\
\hline $\mathrm{H}$ & -3.971007249031 & 2.555578736230 & -1.302596524228 \\
\hline $\mathrm{H}$ & 4062987625 & 4.744584215538 & 5194297 \\
\hline
\end{tabular}




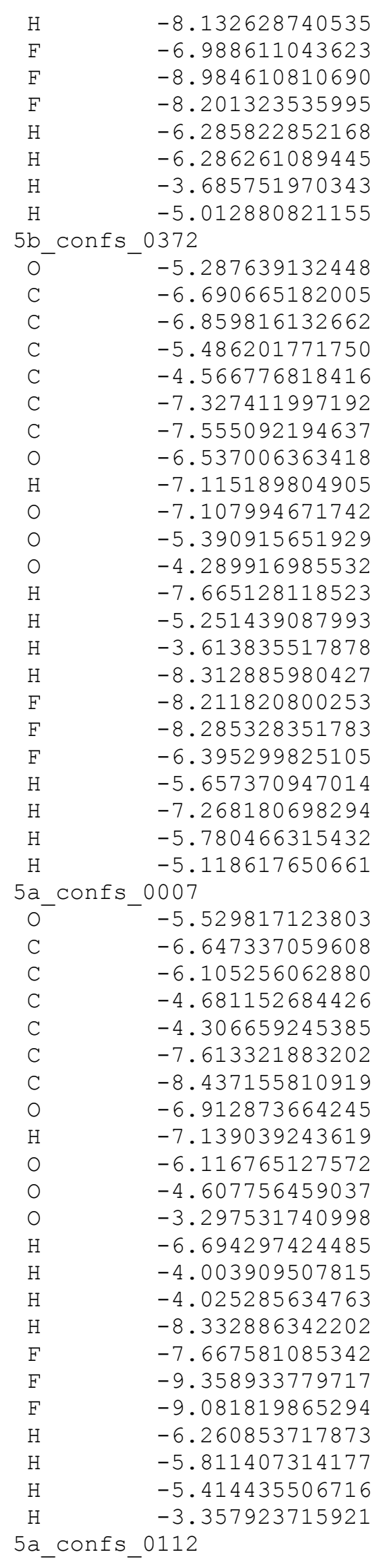

4.393955351138

7.013533912202

6.658311950012

5.422684415589

6.435696705192

1.130638738827

2.833524494706

5.028388387400

3.752902904798

3.509471109311

2.193260677041

2.009959599468

2.605846555146

4.730753054618

5.882497831610

5.180958895665

3.372944302341

1.181044956494

2.679146607707

1. 722314771568

2. 240017050562

0.957333174925

2. 958098550147

4.471125473649

6.889131398376

5.480481330806

6.370688371751

5.363506237318

0.341193763635

3.565637735750

1. 325685647314

3.873730129847

3.209489705704

2. 313662113372

2.830699608420

3.291952184935

4.293888888742

4.907927964125

5.314030887685

2. 563915210320

0.989395815842

3.921332900847

4.220316686134

2.404536033016

2.057178493208

2.458779261596

3.864066383814

5.595700114924

5.751493232253

3.955836717760

5.651350350600

0.384681134409

4.459899622742

4.736136559706
$-0.416300594260$

$-2.001533328424$

$-1.250270639163$

$-2.846094999961$

0.211949335957

$-0.427301308230$

1. 454450490412

1.272999235916

$-1.120659028040$

$-1.044655964751$

$-0.256530715649$

0.405146730474

$-0.672735175345$

$-0.360312154258$

$-1.335506429840$

0.710448373993

$-2.045829157007$

$-1.225956996002$

1.634666020492

$-1.707853577027$

0.486390710358

0.584860463976

$-0.276025560928$

0.038257383183

$-0.747056173280$

$-2.389325844299$

$-1.799172412562$

0.338593490363

$-0.778857792407$

1.563662001317

$-2.016548192760$

$-1.262959039186$

$-0.693178379012$

0.445729520250

0.642412379416

$-0.775670311372$

$-0.194627776333$

$-1.322845879430$

0.474890539882

$-1.430008864821$

$-0.048403071044$

1.535972813310

$-0.801529850941$

1.368441906690

1.014219485250

$-1.427875666476$

0.508877739113

$-2.177823645211$

$-0.840924713974$

$-2.017493892590$

$-0.163839822211$

0.638008650190

1. 465454605175

0.018173273325 


\begin{tabular}{|c|c|}
\hline \multirow{2}{*}{\multicolumn{2}{|c|}{-5.011862915262}} \\
\hline & \\
\hline $\mathrm{C}$ & -6.163893948659 \\
\hline $\mathrm{C}$ & -5.741253156214 \\
\hline $\mathrm{C}$ & -4.210835656213 \\
\hline $\mathrm{C}$ & -3.997566314692 \\
\hline $\mathrm{C}$ & -7.296375344986 \\
\hline $\mathrm{C}$ & -8.633677225212 \\
\hline 0 & -7.439945034973 \\
\hline $\mathrm{H}$ & -6.422446954456 \\
\hline 0 & -6.166208325226 \\
\hline O & -3.548060117671 \\
\hline 0 & -4.157410340886 \\
\hline $\mathrm{H}$ & -6.074298010941 \\
\hline $\mathrm{H}$ & -3.863335802877 \\
\hline $\mathrm{H}$ & -3.040207564528 \\
\hline $\mathrm{H}$ & -7.107454765722 \\
\hline $\mathrm{F}$ & -8.599612162684 \\
\hline $\mathrm{F}$ & -8.939523623172 \\
\hline $\mathrm{F}$ & -9.631538097265 \\
\hline $\mathrm{H}$ & -6.549500016399 \\
\hline $\mathrm{H}$ & -7.086261591373 \\
\hline $\mathrm{H}$ & -3.665737211643 \\
\hline $\mathrm{H}$ & -3.914495818946 \\
\hline \multicolumn{2}{|c|}{ 5a_confs_0719 } \\
\hline 0 & -6.767174773969 \\
\hline $\mathrm{C}$ & -6.673380182807 \\
\hline $\mathrm{C}$ & -5.215563612775 \\
\hline $\mathrm{C}$ & -4.493816646502 \\
\hline $\mathrm{C}$ & -5.581282572945 \\
\hline $\mathrm{C}$ & -7.717030916440 \\
\hline $\mathrm{C}$ & -7.874259586277 \\
\hline 0 & -8.977138127684 \\
\hline $\mathrm{H}$ & -6.926033580457 \\
\hline 0 & -4.779876173936 \\
\hline 0 & -3.290536908239 \\
\hline 0 & -5.572005692119 \\
\hline $\mathrm{H}$ & -5.108600715692 \\
\hline $\mathrm{H}$ & -4.271533892783 \\
\hline $\mathrm{H}$ & -5.479357954471 \\
\hline $\mathrm{H}$ & -7.391299459048 \\
\hline $\mathrm{F}$ & -6.742694377835 \\
\hline $\mathrm{F}$ & -8.140857957304 \\
\hline $\mathrm{F}$ & -8.861593636378 \\
\hline $\mathrm{H}$ & -8.844418399441 \\
\hline $\mathrm{H}$ & -3.914969085077 \\
\hline $\mathrm{H}$ & -3.509448520296 \\
\hline $\mathrm{H}$ & -6.168127227527 \\
\hline \multicolumn{2}{|c|}{ 6a_confs_1150 } \\
\hline 0 & -2.106236416613 \\
\hline $\mathrm{C}$ & -2.870828984700 \\
\hline $\mathrm{C}$ & -3.791254006080 \\
\hline $\mathrm{C}$ & -2.870998179811 \\
\hline $\mathrm{C}$ & -1.946726124011 \\
\hline $\mathrm{C}$ & -1.194992574020 \\
\hline $\mathrm{C}$ & -3.621228182393 \\
\hline 0 & -4.716758609588 \\
\hline 0 & -3.555942149743 \\
\hline
\end{tabular}

4.923857703735

4.067174331487

2.876696405228

2. 962618742441

4.472566240753

4.933394783035

4.214444103496

6.114102956133

3.715808319300

1.635303829021

2. 363797490371

4.974423770988

3.022446328565

2. 477646888799

4.778864193054

5.141308621482

3.081659559669

3. 845321818207

4.964807481029

6.430668128692

1.490133336963

2.931623231460

5.906631736094

4.195803000722

4.553926785675

4.325663242712

4.195774142744

3.561578261094

3. 707411486634

4.126007081931

3.855498160912

5.615451803447

5.412314443751

3.482666540243

2. 189654377157

3. 378626982384

5.201575041597

3.722580862864

2.655507145220

3. 873081932610

5.431839397099

3.448167737509

3.845455416144

5.204607313178

2.541358583876

1.707450260496

5.107839537756

5.447813821415

4.280239977891

3.094853695229

2.798878438810

4.060481266969

6.719587663527

3.980130221961

1.885701244545
$-1.308448725543$

$-1.188468294766$

$-0.307269603248$

$-0.342868093998$

$-0.444252719070$

$-0.632900419674$

$-0.713517953404$

$-1.371799938112$

$-2.193501005043$

$-0.806221151438$

0.729054940122

0.860910132080

0.730113184261

$-1.260751934246$

$-0.873409797929$

0.432048591440

0.034838407139

$-1.961981795329$

$-0.246147668955$

$-1.577642496869$

$-0.556433352694$

1. 503340263502

0.893009431776

1.364448447711

$-0.030593099503$

$-0.439530746724$

0.910248255257

1. 773459139402

$-0.762698405067$

$-2.217341775953$

$-0.167766492986$

$-0.122124131108$

$-1.217924503780$

0.864006029149

1.457479974006

$-0.988305756404$

1. 280565907398

2.849882699449

$-0.766854895164$

$-2.902834676484$

$-2.340955989068$

$-2.812975505138$

0.789756555325

$-1.590163933829$

0.810405123470

2.041817780041

$-1.146104096124$

$-0.004641955739$

0.366648480114

0.691053014886

$-0.482962304439$

$-0.915363853100$

$-0.369705710413$

$-0.648216905878$

0.939078914053 


\begin{tabular}{|c|c|}
\hline & \\
\hline 0 & -0.965592238455 \\
\hline 0 & -0.287338423799 \\
\hline $\mathrm{H}$ & -2.215947628808 \\
\hline $\mathrm{H}$ & -4.376121145672 \\
\hline $\mathrm{H}$ & -2.252485723250 \\
\hline $\mathrm{H}$ & -2.553697446625 \\
\hline $\mathrm{H}$ & -0.691837932124 \\
\hline $\mathrm{F}$ & -2.783632698340 \\
\hline $\mathrm{F}$ & -4.515408407107 \\
\hline $\mathrm{F}$ & -4.281356349964 \\
\hline $\mathrm{H}$ & -4.319679585617 \\
\hline $\mathrm{H}$ & -4.285661688649 \\
\hline $\mathrm{H}$ & -1.416531501652 \\
\hline $\mathrm{H}$ & 0.136255997022 \\
\hline $5 b$ & 0928 \\
\hline 0 & -5.770201626398 \\
\hline C & -6.341481314959 \\
\hline $\mathrm{C}$ & -6.204321738938 \\
\hline $\mathrm{C}$ & -4.814534499422 \\
\hline $\mathrm{C}$ & -4.680573622696 \\
\hline $\mathrm{C}$ & -7.792766301090 \\
\hline $\mathrm{C}$ & -7.915756958439 \\
\hline 0 & -8.477071883314 \\
\hline $\mathrm{H}$ & -5.780586549372 \\
\hline 0 & -6.363313241298 \\
\hline 0 & -4.721665738717 \\
\hline 0 & -3.456070164630 \\
\hline $\mathrm{H}$ & -6.943762974862 \\
\hline $\mathrm{H}$ & -4.070522370955 \\
\hline $\mathrm{H}$ & -4.768961123976 \\
\hline $\mathrm{H}$ & -8.288906659982 \\
\hline $\mathrm{F}$ & -7.223201052783 \\
\hline $\mathrm{F}$ & -7.452901806541 \\
\hline $\mathrm{F}$ & -9.192026467237 \\
\hline $\mathrm{H}$ & -7.922130573386 \\
\hline $\mathrm{H}$ & -6.394434417050 \\
\hline $\mathrm{H}$ & -3.795917449681 \\
\hline $\mathrm{H}$ & -3.384891464276 \\
\hline & 0107 \\
\hline 0 & -2.321638191668 \\
\hline $\mathrm{C}$ & -3.365720221469 \\
\hline $\mathrm{C}$ & -3.460854886581 \\
\hline $\mathrm{C}$ & -2.125345301949 \\
\hline $\mathrm{C}$ & -0.985548234644 \\
\hline $\mathrm{C}$ & -1.052434429095 \\
\hline C & -4.632104655644 \\
\hline 0 & -3.606040997386 \\
\hline 0 & -1.979390777133 \\
\hline 0 & 0.265426114539 \\
\hline 0 & -0.790033733993 \\
\hline $\mathrm{H}$ & -3.221734209136 \\
\hline $\mathrm{H}$ & -4.283905664614 \\
\hline $\mathrm{H}$ & -2.011093430056 \\
\hline $\mathrm{H}$ & -1.073578536001 \\
\hline $\mathrm{H}$ & -0.356267153344 \\
\hline $\mathrm{F}$ & -5.670041932496 \\
\hline $\mathrm{F}$ & -4.95065847079 \\
\hline
\end{tabular}

1.845904806265

4.458649641696

5.699175525324

4.544063451166

3. 370753257312

2. 438183803488

3.903663034963

7.758352637710

7.014472677864

6.615536426364

4.156038642688

2.027238041984

1.085086984474

3.657355200598

3.925681412015

3.732272355499

2.230532020569

1.969963305265

3.028200120560

4.196754129729

5.718501983866

3.679080063760

4. 274722722223

1. 922159125467

0.635010592855

3.678635766500

1. 708793325435

2.191815453697

2.594059143991

3. 836844632048

6.253499186146

6.264542488748

6.100002197965

3.833703339141

0.964056981796

0.398615194469

4.388554458256

5.894053506877

4.968363204236

3.997667959562

3. 251604075624

4.255494476047

5.296417984880

5.777805465453

4.636272612984

2.372819608422

3.607920162669

4.694865758633

4.389818832236

3.288427686371

2.707277970817

4.762443471456

6.118478005707

4.947003953597

6.531383212747
$-0.127269727955$

0.072997813240

0.838868265090

1.256746320688

1.559950997282

$-1.324926815459$

$-1.875292030305$

$-0.476034113688$

0.590139347728

$-1.529250139224$

$-1.512280418687$

1. 552853262574

0.262915371750

0.415796283605

0.172945707189

$-1.119141641086$

$-1.335069553959$

$-0.771421795880$

0.337346571359

$-1.083024158544$

$-1.094700955159$

0.021472331136

$-1.892759634417$

$-2.696019362977$

$-0.329377976450$

0.168298789224

$-0.709653410664$

$-1.548212594380$

1. 340340716547

$-1.991371153884$

$-2.116594029588$

0.041040665815

$-1.231824468343$

0.800638891778

$-2.797625468522$

$-0.204658309864$

0.818370840670

0.188521357394

0.364000694838

$-0.827903669203$

$-0.857572141897$

$-0.985479071181$

0.136688372902

0.576756324819

$-2.071056639533$

$-1.952535260957$

$-0.850686604769$

1. 376963452658

1.287594607724

$-0.641965182646$

0.092232745887

$-1.953290967890$

$-0.058111511534$

0.790808819394

$-0.496071468714$ 


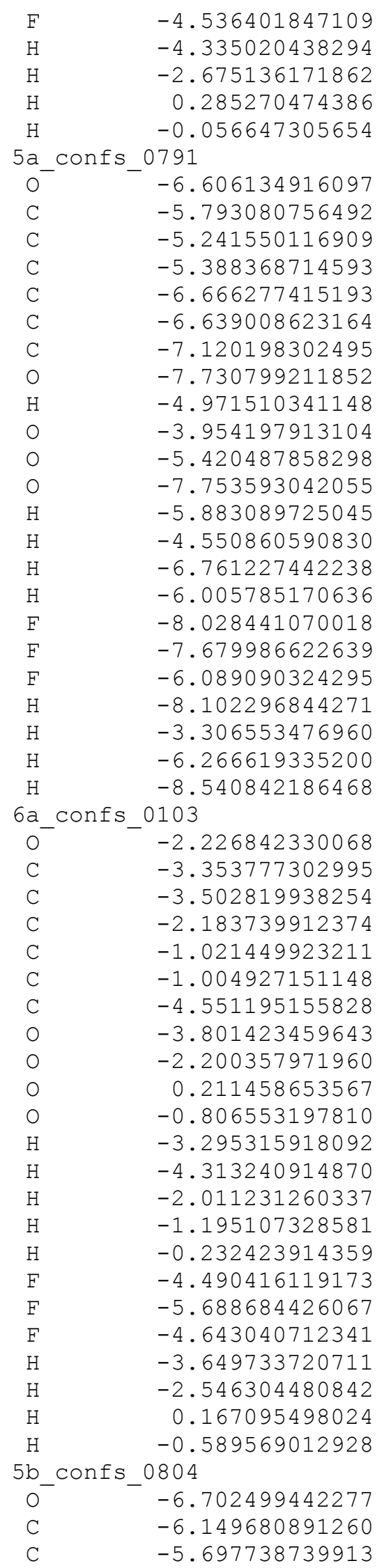

6.594402503436

5.266968801109

1.705994325942

2.871798050840

4.075418370354

3.515427193637

4.028882285761

2.805271564963

1. 685732262690

2.115567149782

4. 923030046854

6.185219012476

4.242377499244

4.608918796720

2.998372215884

0.384824431248

1.684928039445

2.578913819248

1.737912096614

1.738614163959

5.264338753079

5.923681815186

7.041002998140

6.825866173047

3. 654668453960

3.016358615404

0.266201208111

1.571891404547

5.780973096889

4.952407387565

3.894406294197

3.118276981773

4.085994221072

5.068406760558

5.887433680729

4.521934807088

2. 291924548124

3.414667011144

4. 346930320204

4.433805831505

3.209183237827

2. 522335611971

4.675135779783

5.828668287721

6.605884643205

5.166920319363

6.744456474184

3.873689717273

1.419767375137

2.790210499973

4.957787112716

4.446962135682

4.290087863200

2. 816916372689
1. 624084070922

$-2.046353930602$

$-1.944642213213$

$-1.476523411982$

1.245341627583

0.658518637877

$-0.408035350840$

$-1.162582051763$

$-0.124090152031$

0.601321817598

$-1.311022576261$

$-0.598369122088$

$-1.856083082989$

0.029167806498

$-1.686961280313$

$-0.625068004610$

$-0.192726869228$

$-2.021196601574$

0.584213210623

1. 622424849458

$-2.139345199734$

0.350161319654

$-1.464525154759$

$-0.011434324036$

$-1.178907753689$

$-0.971685500161$

$-1.079357058630$

0.351582440995

0.194682603412

0.378269818698

$-0.715667481197$

$-0.820169337241$

$-0.996765949932$

0.169104317455

0.475621388172

$-1.937435013077$

$-1.981158406821$

$-1.047960168321$

1. 348677112590

1.345783951397

$-0.420461876609$

0.084458766457

$-1.907780614659$

0.021884296710

1. 611417413620

0.521739397166

$-0.535793289408$

$-2.639232627263$

$-1.762886992588$

$-1.783023272251$

2.062395963691

0.993838134428

$-0.330299040325$

$-0.430227727175$ 


\begin{tabular}{|c|c|}
\hline & \\
\hline $\mathrm{C}$ & -6.526776795889 \\
\hline $\mathrm{C}$ & -6.554149190226 \\
\hline $\mathrm{C}$ & -7.273887710940 \\
\hline C & -6.743914579448 \\
\hline 0 & -7.883977869052 \\
\hline $\mathrm{H}$ & -5.292358619944 \\
\hline 0 & -4.315090049038 \\
\hline 0 & -7.866580603027 \\
\hline O & -5.309342542745 \\
\hline $\mathrm{H}$ & -5.930105232273 \\
\hline $\mathrm{H}$ & -6.046297273058 \\
\hline $\mathrm{H}$ & -7.391523381862 \\
\hline $\mathrm{H}$ & -8.001586043038 \\
\hline $\mathrm{F}$ & -6.172554486825 \\
\hline $\mathrm{F}$ & -5.816590856628 \\
\hline $\mathrm{F}$ & -7.725822853209 \\
\hline $\mathrm{H}$ & -8.035061264470 \\
\hline $\mathrm{H}$ & -4.108254697885 \\
\hline $\mathrm{H}$ & -7.908780712980 \\
\hline $\mathrm{H}$ & -5.240426164012 \\
\hline $5 b$ & 0792 \\
\hline 0 & -5.838318208928 \\
\hline $\mathrm{C}$ & -6.042818608683 \\
\hline $\mathrm{C}$ & -5.451089529574 \\
\hline $\mathrm{C}$ & -5.437282397768 \\
\hline $\mathrm{C}$ & -5.115551290876 \\
\hline $\mathrm{C}$ & -7.557103088601 \\
\hline $\mathrm{C}$ & -7.899540277650 \\
\hline 0 & -8.055300661862 \\
\hline $\mathrm{H}$ & -5.507529223959 \\
\hline 0 & -4.168208518909 \\
\hline 0 & -6.720678434957 \\
\hline 0 & -3.727966325745 \\
\hline $\mathrm{H}$ & -6.078812164440 \\
\hline $\mathrm{H}$ & -4.674191085373 \\
\hline $\mathrm{H}$ & -5.434637764795 \\
\hline $\mathrm{H}$ & -8.061262011605 \\
\hline $\mathrm{F}$ & -7.301592957374 \\
\hline $\mathrm{F}$ & -9.217224115663 \\
\hline $\mathrm{F}$ & -7.502301598512 \\
\hline $\mathrm{H}$ & -7.667040238834 \\
\hline $\mathrm{H}$ & -3.559494787663 \\
\hline $\mathrm{H}$ & -6.938701857922 \\
\hline $\mathrm{H}$ & -3.516354850306 \\
\hline & 0399 \\
\hline 0 & -6.535792401499 \\
\hline $\mathrm{C}$ & -5.640314987970 \\
\hline $\mathrm{C}$ & -4.932818551231 \\
\hline $\mathrm{C}$ & -5.73694591213 \\
\hline $\mathrm{C}$ & -6.186639086564 \\
\hline $\mathrm{C}$ & -6.423166875225 \\
\hline C & -7.266661992198 \\
\hline 0 & -7.23359746202 \\
\hline $\mathrm{H}$ & -4.88169025130 \\
\hline 0 & -3.605622868626 \\
\hline 0 & -6.86665222913 \\
\hline 0 & -7.30940393554 \\
\hline
\end{tabular}

2.148665036859

3.256174139943

4.707722926408

4.998938050019

5.892890639676

4.962817409311

2. 653907854647

1. 939022202378

3.225050758801

2.389698103914

1.240453696292

3.189631264784

3.887930764125

3.897455838725

5.964664941967

5.375507111050

5.803930132491

2.890065327273

1.191722131354

4.004785298410

5.254452412558

4.410253934599

3.033225991465

3.092426327695

4.561907667642

4.436287587611

3. 947577433415

5.742829432172

4.840692232596

2.825919225943

2.875370953958

4.710891836772

2.214424244050

2.433833871365

4.942299990116

3.767509417839

4.676260965059

3.987386083894

2.668417640884

6.146032472944

3.451814143864

1.938173256509

5.653012877049

3.691341360568

4.046523529495

2.740854940190

1.657803809248

2.412041554036

4.700276531494

5.878110219398

3.821015436999

4.742967215373

2.804065160282

1.334456352703

1.788199014337
0.683169131577

1.726233410003

$-1.290304991522$

$-2.686015886964$

$-0.860596045547$

$-0.437410040783$

$-0.235267029027$

0.289217915057

2. 386560173468

$-1.408832527804$

1.061409937356

2. 426323818117

$-1.380826030264$

$-3.217440499234$

$-2.683736105766$

$-3.511065165434$

0.090341089182

0.681029351583

$-0.318045761799$

2. 951943890872

0.386836631606

$-0.765975999229$

$-0.394410583547$

1.144992392889

1.374015525415

$-1.023238904226$

$-2.422393824112$

$-0.944594627508$

$-1.618739296158$

$-0.930772147055$

1. 692594658280

1.176989880017

$-0.754575272423$

1. 572478594828

2. 348312932123

$-0.310244325146$

$-3.373176677170$

$-2.643289284236$

$-2.590114245097$

$-0.156103799151$

$-0.511390940161$

1.636473833090

1. 172325476971

0.587818543806

$-0.465268598527$

$-0.900500440212$

$-0.174534523478$

1.075965519858

$-1.601353865697$

$-1.110107838325$

$-2.321649496948$

$-0.090162979076$

$-0.415033773748$

$-0.957065392783$

1.609499084145 


\begin{tabular}{|c|c|}
\hline & \\
\hline $\mathrm{H}$ & -4.970958762680 \\
\hline $\mathrm{H}$ & -5.132342944351 \\
\hline $\mathrm{H}$ & -5.378280920626 \\
\hline $\mathrm{H}$ & -5.694301430709 \\
\hline $\mathrm{F}$ & -7.712352945736 \\
\hline $\mathrm{F}$ & -6.528962716936 \\
\hline $\mathrm{F}$ & -8.334485025710 \\
\hline $\mathrm{H}$ & -7.473560086545 \\
\hline $\mathrm{H}$ & -3.088626180109 \\
\hline $\mathrm{H}$ & -7.557575557744 \\
\hline $\mathrm{H}$ & -7.743246875391 \\
\hline $6 \mathrm{~b}$ & 0141 \\
\hline $0^{-}$ & -2.421388409577 \\
\hline $\mathrm{C}$ & -3.546667435257 \\
\hline $\mathrm{C}$ & -3.093842851599 \\
\hline $\mathrm{C}$ & -2.284222966628 \\
\hline $\mathrm{C}$ & -1.177942203170 \\
\hline $\mathrm{C}$ & -1.731958268663 \\
\hline $\mathrm{C}$ & -4.338179965097 \\
\hline 0 & -2.305274849419 \\
\hline 0 & -1.773106359318 \\
\hline 0 & -0.626534959014 \\
\hline 0 & -0.653927665385 \\
\hline $\mathrm{H}$ & -4.212101388594 \\
\hline $\mathrm{H}$ & -3.958898262934 \\
\hline $\mathrm{H}$ & -2.942231609479 \\
\hline $\mathrm{H}$ & -0.415915981735 \\
\hline $\mathrm{H}$ & -2.436855038389 \\
\hline $\mathrm{F}$ & -3.584773540030 \\
\hline $\mathrm{F}$ & -4.871019716997 \\
\hline $\mathrm{F}$ & -5.346677273209 \\
\hline $\mathrm{H}$ & -1.807908103448 \\
\hline $\mathrm{H}$ & -1.424994852181 \\
\hline $\mathrm{H}$ & 0.113621194767 \\
\hline $\mathrm{H}$ & -0.966499494643 \\
\hline $6 b_{3}$ & 0119 \\
\hline 0 & -2.535576507138 \\
\hline $\mathrm{C}$ & -3.632912484295 \\
\hline $\mathrm{C}$ & -3.209658030683 \\
\hline $\mathrm{C}$ & -2.434446144453 \\
\hline $\mathrm{C}$ & -1.297774446668 \\
\hline $\mathrm{C}$ & -1.827982254030 \\
\hline $\mathrm{C}$ & -4.395133519260 \\
\hline 0 & -2.312261307974 \\
\hline 0 & -1.918011276204 \\
\hline 0 & -0.716476417832 \\
\hline 0 & -0.764806891219 \\
\hline $\mathrm{H}$ & -4.340901163537 \\
\hline $\mathrm{H}$ & -4.103810688491 \\
\hline $\mathrm{H}$ & -3.112292252062 \\
\hline $\mathrm{H}$ & -0.559810215743 \\
\hline $\mathrm{H}$ & -2.526785064200 \\
\hline $\mathrm{F}$ & -5.482433828576 \\
\hline $\mathrm{F}$ & -3.658322546311 \\
\hline $\mathrm{F}$ & -4.805094813977 \\
\hline $\mathrm{H}$ & -2.687560219403 \\
\hline $\mathrm{H}$ & -1.538752649380 \\
\hline
\end{tabular}

2.595126739061

0.771514201833

2.495633644260

5.137652907014

6.601025673263

6.703150498377

5.500074480543

3.058212583498

2. 085335474072

1. 023131973837

2. 387486700120

5.555101315458

5.057644743444

4.141881759217

2. 971608448423

3. 491404862258

4.507981168287

6.273948720782

4.832494214920

2.181771236359

2.399718229801

5.053721423987

4.497982605141

3.742835403209

2. 324059055614

4.009395425432

4.013136901963

7.153039085378

6.917326459497

5.893923851825

5.534536175771

2. 784889041048

2.719861633394

5.640938238793

5.609530683886

5.096321285961

4.087758232399

2. 977064854230

3.548921651241

4.607537617971

6.284908008907

4.611853230653

2.041991916176

2. 520816747012

5.215818282390

4.588229894650

3.670103867940

2.418663574426

4.041217112373

4.147054249280

5.854999121246

7.001502126530

7.113585272293

5.373445251163

2. 545633259219
$-1.985859992348$

0.051380123903

1. 816676375970

$-2.296739573624$

$-2.149867897042$

$-0.339045319483$

$-0.402454349615$

$-1.769136298104$

$-0.795891292424$

$-0.352116175096$

2. 229448158845

0.365312188263

$-0.324103606828$

$-1.458872173235$

$-0.875261797997$

0.045282458472

1.039799550332

$-0.784121630923$

$-2.410989787430$

$-1.914690968634$

0.736054140766

1.713038006547

0.352970168906

$-1.997156819890$

$-0.280708567666$

$-0.559606235816$

1.731450501620

$-1.453212440150$

0.265201878770

$-1.582948590753$

$-1.967210528064$

$-2.587557020518$

1. 266746250133

2. 412185024096

0.416074780458

$-0.282971264443$

$-1.361784511828$

$-0.658784065856$

0.194556045674

1.150284190581

$-0.844609609928$

$-2.318218114366$

$-1.579791771405$

0.958357914342

1.776163663549

0.395793122604

$-1.844871575202$

$-0.000358087788$

$-0.455754162876$

1.872985200925

$-1.511947409483$

$-1.713435960220$

0.116420904678

$-2.774894159346$

$-2.315398826866$ 


\begin{tabular}{|c|c|}
\hline $\mathrm{H}$ & -0.515454760606 \\
\hline $\mathrm{H}$ & -1.095542517957 \\
\hline \multicolumn{2}{|c|}{ 5b confs 0373} \\
\hline 0 & -5.292589622465 \\
\hline $\mathrm{C}$ & -6.596277680504 \\
\hline $\mathrm{C}$ & -6.394299785912 \\
\hline $\mathrm{C}$ & -5.009576803381 \\
\hline $\mathrm{C}$ & -4.286351620631 \\
\hline $\mathrm{C}$ & -7.396613506167 \\
\hline $\mathrm{C}$ & -8.107189211359 \\
\hline 0 & -6.570670207806 \\
\hline $\mathrm{H}$ & -7.092232316782 \\
\hline 0 & -6.276134027462 \\
\hline 0 & -5.059437627464 \\
\hline O & -3.764762527647 \\
\hline $\mathrm{H}$ & -7.16270658338 \\
\hline $\mathrm{H}$ & -4.530747382862 \\
\hline $\mathrm{H}$ & -3.471676874149 \\
\hline $\mathrm{H}$ & -8.179420083201 \\
\hline $\mathrm{F}$ & -7.230625160264 \\
\hline $\mathrm{F}$ & -8.845559587 \\
\hline $\mathrm{F}$ & -8.929354138318 \\
\hline $\mathrm{H}$ & -5.816043416504 \\
\hline $\mathrm{H}$ & -7.116234714284 \\
\hline $\mathrm{H}$ & -5.52650134 \\
\hline $\mathrm{H}$ & -4.426995777891 \\
\hline \multicolumn{2}{|c|}{ 6b_confs_0143 } \\
\hline 0 & -2.487331955800 \\
\hline $\mathrm{C}$ & -3.530547614523 \\
\hline $\mathrm{C}$ & -3.027550821740 \\
\hline $\mathrm{C}$ & -2.189883764270 \\
\hline $\mathrm{C}$ & -1.114233370309 \\
\hline $\mathrm{C}$ & -1.74584024185 \\
\hline $\mathrm{C}$ & -4.339339562160 \\
\hline 0 & -2.152361800109 \\
\hline 0 & -1.592239692368 \\
\hline 0 & -0.417471085267 \\
\hline 0 & -0.83385358264 \\
\hline $\mathrm{H}$ & -4.22948906809 \\
\hline $\mathrm{H}$ & -3.88522042681 \\
\hline $\mathrm{H}$ & -2.835438519292 \\
\hline $\mathrm{H}$ & -0.436312863969 \\
\hline $\mathrm{H}$ & -2.42212235933 \\
\hline $\mathrm{F}$ & -3.609792789 \\
\hline $\mathrm{F}$ & -4.84100057614 \\
\hline $\mathrm{F}$ & -5.36720192879 \\
\hline $\mathrm{H}$ & -2.582596799414 \\
\hline $\mathrm{H}$ & -1.25587115890 \\
\hline $\mathrm{H}$ & -0.20113156276 \\
\hline $\mathrm{H}$ & -0.13426845600 \\
\hline \multicolumn{2}{|c|}{ 6b confs 0448} \\
\hline 0 & -2.21036939573 \\
\hline $\mathrm{C}$ & -3.46647049998 \\
\hline $\mathrm{C}$ & -3.36949598742 \\
\hline $\mathrm{C}$ & -2.43698065766 \\
\hline $\mathrm{C}$ & -1.10837098056 \\
\hline 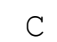 & -1.35364536562 \\
\hline
\end{tabular}

1.793860950323

5.839582809732

4.179334643300

3.609315589553

2.090926658502

2.022362053127

3.163567349583

4.300382805043

5.549669539075

4.647341697463

3.794861652770

1.436223876834

2.203641963394

2.793752244596

1.623902869939

1.060581651817

3.594869696078

3.637134369983

6.463847076146

6.119159934768

5.243701384468

5.129845306409

1. 462088406781

3.025723206645

2. 270766023726

5.571831477033

5.017575021275

4.060517511007

2. 987354855692

3. 626312793072

4.591585195816

6.177159640823

4.663530519212

2.113729942491

2.634564384175

5.246513672408

4.456348985942

3.597425501144

2. 367337081603

4.212509859207

4.052538260144

6.962686537899

6.947696544588

5.703309843403

5.390252720562

2.657958639568

1.931543487646

5.614717525288

5.910832490180

5.295029631697

3.877140349913

3.041507419668

3.776433121615

5.205818341827
0.353745421176

2.433638275621

$-1.214616780675$

$-1.197007553202$

$-1.001555944334$

$-0.350172537456$

$-1.084791805592$

$-0.067467473220$

$-0.577274455059$

1.012102023977

$-2.157952559726$

$-2.258026022044$

1.039644327147

$-2.313262472326$

$-0.375310919332$

$-0.542018077421$

$-0.500535629073$

0.314438045427

$-1.017812246111$

0.379873311687

$-1.599302715095$

0.633066648435

$-2.730492294668$

1.261341047366

$-2.789865918705$

0.393054320897

$-0.355597128182$

$-1.447617741141$

$-0.758265821975$

0.124381430838

1. 125379651533

$-0.914353058288$

$-2.380056134048$

$-1.690441519091$

0.834346969499

1. 916523113657

0.289530016877

$-1.954070691590$

$-0.123042475757$

$-0.519576943655$

1.805424690555

$-1.728810618519$

0.048809744918

$-1.645255772223$

$-2.845331897031$

$-2.418047603212$

0.206211374275

1.361306091663

$-0.149792912735$

$-0.235437760904$

$-0.815059850277$

0.071714860726

0.258913162872

0.736172175019 


\begin{tabular}{|c|c|}
\hline \multirow{2}{*}{\multicolumn{2}{|c|}{-4.356997773557}} \\
\hline & \\
\hline 0 & -2.778322579293 \\
\hline 0 & -2.268807303798 \\
\hline 0 & -0.355594121469 \\
\hline 0 & -0.126670069692 \\
\hline $\mathrm{H}$ & -3.945586517511 \\
\hline $\mathrm{H}$ & -4.365925003576 \\
\hline $\mathrm{H}$ & -2.894292548110 \\
\hline $\mathrm{H}$ & -0.585417810346 \\
\hline $\mathrm{H}$ & -1.822658745339 \\
\hline $\mathrm{F}$ & -4.467956566519 \\
\hline $\mathrm{F}$ & -5.591937512106 \\
\hline $\mathrm{F}$ & -3.905047187002 \\
\hline $\mathrm{H}$ & -3.290208030609 \\
\hline $\mathrm{H}$ & -2.043811268921 \\
\hline $\mathrm{H}$ & 0.496910824894 \\
\hline $\mathrm{H}$ & -0.229344900057 \\
\hline \multicolumn{2}{|c|}{ 6b confs 1018} \\
\hline 0 & -1.712727678324 \\
\hline $\mathrm{C}$ & -2.876315122880 \\
\hline $\mathrm{C}$ & -3.837321968888 \\
\hline $\mathrm{C}$ & -3.068948868980 \\
\hline $\mathrm{C}$ & -1.767952801131 \\
\hline $\mathrm{C}$ & -0.953598912384 \\
\hline $\mathrm{C}$ & -3.511180250405 \\
\hline 0 & -4.276039926146 \\
\hline 0 & -3.837337660514 \\
\hline 0 & -0.962156515181 \\
\hline 0 & 0.206910117258 \\
\hline $\mathrm{H}$ & -2.636292780373 \\
\hline $\mathrm{H}$ & -4.689408333288 \\
\hline $\mathrm{H}$ & -2.829550467776 \\
\hline $\mathrm{H}$ & -1.997378502094 \\
\hline $\mathrm{H}$ & -0.725263052606 \\
\hline $\mathrm{F}$ & -2.698132915589 \\
\hline $\mathrm{F}$ & -4.634738606746 \\
\hline $\mathrm{F}$ & -3.865437952901 \\
\hline $\mathrm{H}$ & -4.716720617091 \\
\hline $\mathrm{H}$ & -4.292682094313 \\
\hline $\mathrm{H}$ & -1.504102649459 \\
\hline $\mathrm{H}$ & 0.569377559810 \\
\hline \multicolumn{2}{|c|}{ 6b_confs_0023 } \\
\hline 0 & -2.423650995838 \\
\hline $\mathrm{C}$ & -3.529115587794 \\
\hline $\mathrm{C}$ & -3.070593358137 \\
\hline $\mathrm{C}$ & -2.244377227022 \\
\hline $\mathrm{C}$ & -1.149565531224 \\
\hline $\mathrm{C}$ & -1.744445465402 \\
\hline $\mathrm{C}$ & -4.305582967951 \\
\hline 0 & -2.287421010082 \\
\hline 0 & -1.676353606807 \\
\hline 0 & -0.469386465610 \\
\hline 0 & -0.768495105673 \\
\hline $\mathrm{H}$ & -4.213158023017 \\
\hline $\mathrm{H}$ & -3.937292800261 \\
\hline $\mathrm{H}$ & -2.891558693104 \\
\hline $\mathrm{H}$ & -0.444133501414 \\
\hline
\end{tabular}

6.218463587531

3.839449019335

1.760071632603

3. 082473804725

5.845480519821

5.227319676339

3.415157912351

2.902949770035

3.836246100712

5.186501603539

7. 423829969355

5.692310939204

6.395553811641

4.349199506916

1.869217530655

3.526633817587

6.731379442749

5.434246915296

5.310568852377

4.236489113912

2.915393470616

3.125866550266

4.242564551889

6.690410041515

4.473012885780

1.898904279923

1.967069548346

4.510704965704

5.049283859295

4.133705421751

2. 566326200077

3.433022809887

3.983475947654

7.589380601596

6.637013007783

7.147621623806

5.326891944952

2.296572300377

1.219967815721

3. 663507291478

5.565862060906

5.015273373766

4.009600807542

2.920337228977

3. 557575593275

4.584847892563

6.191859647801

4.608496118877

2.032790402588

2. 604551740282

5.259696006982

4.511012355629

3.556939306487

2. 319430658485

4.093144169752
$-1.049789293011$

$-2.098217666861$

$-0.470653969944$

1.223095764674

0.765121491413

0.757295011462

$-0.823983803232$

1.059681835936

$-0.706766636562$

1.737679021350

$-0.488045477669$

$-1.145673652094$

$-2.304439542030$

$-2.736267768686$

$-1.405445888801$

1. 311560201919

1.133340697436

$-0.550344102032$

0.217787234825

$-0.324127088720$

$-0.400922316439$

$-1.169862690925$

$-0.529859835094$

0.281886780547

$-1.646498781599$

$-1.004220572050$

$-1.147500286969$

$-1.234649615462$

1.263595269094

0.361607297154

0.611516344411

$-2.200637397335$

0.522531970398

0.832430433140

1. 023542562504

$-0.933417751882$

$-1.726323381372$

$-1.761500694285$

$-1.434454155295$

$-1.529579222613$

0.388244044247

$-0.287689593423$

$-1.347991430404$

$-0.652352891142$

0.196742682612

1.150096236027

$-0.863953068452$

$-2.364093514076$

$-1.588450094104$

0.981920012080

1.862457903392

0.413885283747

$-1.840067709052$

0.000097433537

$-0.459310324782$ 


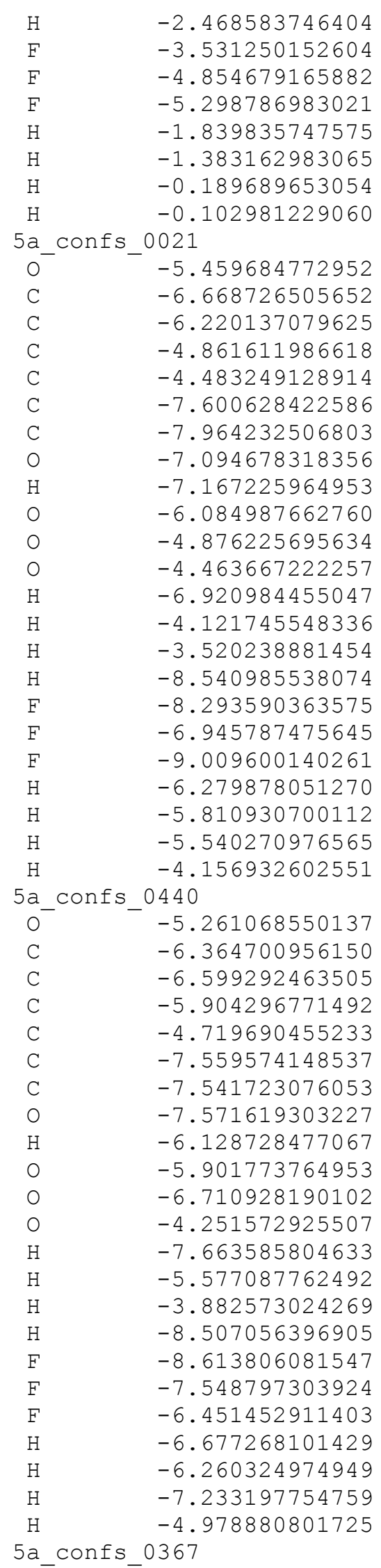

4.097464480867

7.001982779160

6.926353539934

5.745283607082

5.391318628942

2. 567080138234

1.882876904915

4.610822556955

3.577728404861

3. 026101167040

2.027845959801

2.588429589354

3.656807164537

4.091942998774

5.170307315014

4.703775061713

2.510283613040

0.779283149307

3.050097114723

4. 933812887373

1.967989411306

1.783869593072

3.464378383368

3.603227296585

4.637198038786

6.025228386578

5.897303984963

5.186097983724

0.114203408409

3.749601386087

5.571487701583

4.006255350166

3. 266898270482

2.106778586033

2.600844355315

3.396912640415

4.237292752300

4.996165219353

5.154328750943

2.852895537336

0.943412003645

3.436134424527

4.366820354835

1.901935009130

1.764563949974

2.756598837473

3.688924470476

5.785784780836

4.135483663589

5.766209961070

5.539316433066

0.606108458779

4.039256318602

4.609079871655
1.829932927898

$-1.596226452409$

0.109501080158

$-1.649646475041$

$-2.011697706757$

$-2.342762982394$

0.402616688690

2.131547949648

$-1.504473806622$

$-0.986429146962$

0.078956417428

0.574386850323

$-0.496918189577$

$-0.401592494685$

$-1.420080570246$

0.757598191821

$-1.810527180975$

$-0.571120858404$

1.892726638766

0.103390114438

0.921168933389

0.542372230844

$-0.977113926210$

$-0.123802702458$

$-2.606964812478$

$-1.619886577431$

$-1.000201029394$

0.519390536422

0.071077861789

1. 971805273455

$-0.553761753234$

$-0.378161619363$

$-0.870360679650$

0.128667840786

1.391725802147

0.815754432174

$-0.989981026895$

$-2.312586740952$

0.073872176781

$-1.859230325184$

$-0.266141334015$

2.192709397207

1. 667427642218

0.304038222079

2.011064499254

0.524476212138

$-0.968024957008$

$-2.437315370340$

$-3.348723591182$

$-2.425697081196$

0.103835891010

$-1.094832095790$

1.635739410414

2. 262743295366 
$-5.344492558991$

$-5.765330610987$

$-5.901705025474$

$-6.200988000935$

$-5.342201818046$

$-7.078517911114$

$-7.244268746793$

$-7.068403795505$

$-4.982754458858$

$-4.671508623224$

$-7.569096331053$

$-5.932375943345$

$-6.712362685969$

$-5.908722023737$

$-4.307355402303$

$-7.935910866781$

$-7.202252351861$

$-6.268798846389$

$-8.411233919367$

$-6.609423254477$

$-4.451520940185$

$-7.685269223584$

$-5.361506661023$
4.803024602437

4.052113509913

2. 597011100661

2.773882956976

3.992541399569

4.673840951144

4.380410263874

6.067127437426

4.143907441824

1.905888450045

3.072652448581

4. 670173533298

2.059775163804

1.887609282094

3. 712142111782

4.225884909170

3.044576926608

4.925887490207

4.826342220652

6.268811651397

1.741166737138

3.606604745251

5. 387624666148
0.568751307666

$-0.573688256253$

$-0.089631357010$

1.393390705514

1.738578403149

$-1.093991013924$

$-2.576098247507$

$-0.967531482227$

$-1.336520455640$

$-0.167593958737$

1. 529641802690

2. 802215748667

$-0.592527908835$

1. 967394509092

1.967830588546

$-0.573103004820$

$-2.795107427468$

$-3.315062972830$

$-3.047009982561$

$-0.139506966538$

$-1.091419433992$

2. 328334673744

3.098654729274 
CCSD(T)/aug-cc-pVDZ // M06-2X/6-31+G(d,p) (Gas Phase) Energies for Selected Conformers (“6a" = species $A$; “6b" = species $B$; “5a” = species C; “5b” = species D).

$\begin{array}{lll}\text { Conformer } & E(C C S D(T) / a u g-C C-p V D Z)^{a, b} & E_{r e l}{ }^{c} \\ \text { 6a_confs_0106 } & -0.90780413360 \mathrm{E}+03 & \\ \text { 6a_confs_0023 } & -0.90780346400 \mathrm{E}+03 & 0.000 \\ \text { 6a_confs_0051 } & -0.90780317147 \mathrm{E}+03 & 0.420 \\ \text { 5b_confs_0112 } & -0.90780203860 \mathrm{E}+03 & 0.604 \\ \text { 6a_confs_0079 } & -0.90780165856 \mathrm{E}+03 & 1.315 \\ \text { 6a_confs_0067 } & -0.90780157466 \mathrm{E}+03 & 1.553 \\ \text { 6a_confs_0475 } & -0.90780108719 \mathrm{E}+03 & 1.606 \\ \text { 6a_confs_0092 } & -0.90780079635 \mathrm{E}+03 & 1.912 \\ \text { 5b_confs_1170 } & -0.90780063082 \mathrm{E}+03 & 2.094 \\ \text { 6b_confs_0059 } & -0.90780048071 \mathrm{E}+03 & 2.198 \\ \text { 6a_confs_0563 } & -0.90780034424 \mathrm{E}+03 & 2.292 \\ \text { 5b_confs_0242 } & -0.90780005540 \mathrm{E}+03 & 2.378 \\ \text { 5a_confs_0182 } & -0.90780000686 \mathrm{E}+03 & 2.559 \\ \text { 6b_confs_0064 } & -0.90779975790 \mathrm{E}+03 & 2.590 \\ \text { 6a_confs_0146 } & -0.90779963785 \mathrm{E}+03 & 2.746 \\ \text { 6a_confs_0093 } & -0.90779916616 \mathrm{E}+03 & 2.821 \\ \end{array}$

a Hartrees. b Using M06-2X/6-31+G(d,p) geometries. c $\mathrm{kcal} / \mathrm{mol}$. 
M06-2X/6-31+G(d,p) Gibbs Free Energies, Boltzmann Populations, Total Energies, and Zeropoint Energy Corrections for Conformational Ensembles of Systems A-D Within Free Energy Window of $4 \mathrm{kcal} / \mathrm{mol}$; Implicit DMSO Solvent Model (IEFPCM). (“6a" = species A; "6b" = species B; “5a" = species C; “5b” = species D).

\begin{tabular}{|c|c|c|c|c|c|c|}
\hline Conformer & $\Delta \mathrm{G} \quad(298.15 \mathrm{~K})^{\mathrm{a}}$ & $\Delta \mathrm{G}_{\mathrm{rel}}^{\mathrm{b}}$ & Population ${ }^{c}$ & $E_{\text {tot }}{ }^{a}$ & $E_{\text {tot, rel }}{ }^{b}$ & $\mathrm{ZPE} \mathrm{E}^{\mathrm{a}}$ \\
\hline 6a_confs_0106 & -909.301296 & 0 & 0.259941738 & -909.4348561 & 0 & 0.172378 \\
\hline 5a_confs_0182 & -909.300036 & 0.791 & 0.068402551 & -909.4313746 & 2.185 & 0.171522 \\
\hline 6b_confs_0059 & -909.299739 & 0.977 & 0.049972851 & -909.4322762 & 1.619 & 0.171642 \\
\hline 6a_confs_0092 & -909.299466 & 1.148 & 0.037444753 & -909.4324757 & 1.494 & 0.172076 \\
\hline $6 a^{-} \operatorname{confs}{ }^{-} 0475$ & -909.299395 & 1.193 & 0.03470611 & -909.4327485 & 1.323 & 0.17235 \\
\hline $6 a^{-}$confs 0103 & -909.29937 & 1.209 & 0.033781421 & -909.4323828 & 1.552 & 0.171962 \\
\hline 5b_confs_1170 & -909.299352 & 1.22 & 0.03316003 & -909.4320324 & 1.772 & 0.171871 \\
\hline 5a_confs_-0096 & -909.299205 & 1.312 & 0.028390878 & -909.4304398 & 2.771 & 0.171425 \\
\hline 5b_confs_0127 & -909.299199 & 1.316 & 0.028199851 & -909.4317206 & 1.968 & 0.171782 \\
\hline $6 a^{-} \operatorname{confs}{ }^{-} 0146$ & -909.29909 & 1.384 & 0.02514217 & -909.4326648 & 1.375 & 0.172311 \\
\hline $5 \mathrm{~b}^{-}$confs 0242 & -909.298986 & 1.45 & 0.022491825 & -909.4314938 & 2.11 & 0.17186 \\
\hline 6a_confs_0067 & -909.29897 & 1.46 & 0.022115394 & -909.4321325 & 1.709 & 0.17219 \\
\hline 6a_confs_0093 & -909.298877 & 1.518 & 0.020053059 & -909.4318172 & 1.907 & 0.171948 \\
\hline 6a_confs_0079 & -909.298825 & 1.551 & 0.018966693 & -909.4318424 & 1.891 & 0.172027 \\
\hline $6 \mathrm{~b}^{-} \mathrm{confs}-0064$ & -909.29882 & 1.554 & 0.0188709 & -909.4309896 & 2.426 & 0.171428 \\
\hline $5 a^{-} \operatorname{confs}{ }^{-} 0791$ & -909.298696 & 1.632 & 0.016543162 & -909.4301331 & 2.964 & 0.17162 \\
\hline 6a_confs_0129 & -909.2986 & 1.692 & 0.014949907 & -909.4314991 & 2.107 & 0.17196 \\
\hline $6 a^{-}$confs ${ }^{-} 0023$ & -909.298525 & 1.739 & 0.013809802 & -909.4317187 & 1.969 & 0.17223 \\
\hline $6 a^{-}$confs-0105 & -909.298382 & 1.829 & 0.011863623 & -909.4318004 & 1.917 & 0.17225 \\
\hline $6 a^{-}$confs 0051 & -909.298357 & 1.844 & 0.011567043 & -909.4312707 & 2.25 & 0.171922 \\
\hline $6 a^{-}$confs ${ }^{-} 0097$ & -909.298348 & 1.85 & 0.011450497 & -909.431574 & 2.06 & 0.17213 \\
\hline 5a_confs_0814 & -909.298345 & 1.852 & 0.01141191 & -909.4292687 & 3.506 & 0.17115 \\
\hline $5 a^{-}$confs ${ }^{-} 0511$ & -909.298178 & 1.957 & 0.009558577 & -909.4293901 & 3.43 & 0.171365 \\
\hline 5b_confs_0170 & -909.298079 & 2.019 & 0.00860889 & -909.4308181 & 2.534 & 0.172015 \\
\hline $6 \mathrm{~b}^{-}$confs 0141 & -909.298046 & 2.039 & 0.008323139 & -909.4304337 & 2.775 & 0.171614 \\
\hline $5 \mathrm{~b}^{-}$confs ${ }^{-} 0109$ & -909.298027 & 2.051 & 0.008156262 & -909.4306334 & 2.65 & 0.17192 \\
\hline $5 a^{-}$confs 0079 & -909.297934 & 2.11 & 0.007383191 & -909.4291836 & 3.56 & 0.17152 \\
\hline $5 \mathrm{~b}^{-}$confs ${ }^{-} 0112$ & -909.297866 & 2.152 & 0.006877935 & -909.430317 & 2.848 & 0.17202 \\
\hline 5b_confs_0114 & -909.297797 & 2.196 & 0.006385663 & -909.4303267 & 2.842 & 0.17193 \\
\hline $6 b^{-}$confs 0448 & -909.29779 & 2.2 & 0.006342698 & -909.4302034 & 2.92 & 0.17175 \\
\hline $5 \mathrm{~b}^{-}$confs -0204 & -909.297768 & 2.214 & 0.006194582 & -909.430293 & 2.863 & 0.17184 \\
\hline 6b_confs_0119 & -909.297713 & 2.248 & 0.005849112 & -909.4301456 & 2.956 & 0.17175 \\
\hline $6 \mathrm{~b}^{-} \operatorname{confs}{ }^{-} 0053$ & -909.29768 & 2.269 & 0.005645429 & -909.4302374 & 2.898 & 0.171686 \\
\hline $5 \mathrm{~b}^{-}$confs -0509 & -909.297673 & 2.273 & 0.005607444 & -909.4300025 & 3.046 & 0.17171 \\
\hline 5a_confs_0093 & -909.29765 & 2.288 & 0.005467263 & -909.4286353 & 3.904 & 0.1712 \\
\hline $5 a^{-} \operatorname{confs}{ }^{-} 0867$ & -909.297621 & 2.306 & 0.005303663 & -909.4279778 & 4.316 & 0.1710 \\
\hline 5a_confs_0719 & -909.297574 & 2.336 & 0.005041803 & -909.4274198 & 4.666 & 0.1707 \\
\hline $5 a^{-} \operatorname{confs}-0251$ & -909.297563 & 2.342 & 0.004991004 & -909.4295554 & 3.326 & 0.17172 \\
\hline $6 \mathrm{~b}^{-}$confs ${ }^{-} 0714$ & -909.297453 & 2.411 & 0.00444233 & -909.4301351 & 2.962 & 0.1717 \\
\hline 5a_confs_0399 & -909.297422 & 2.431 & 0.004294878 & -909.4284262 & 4.035 & 0.1710 \\
\hline $5 a^{-} \operatorname{confs}{ }^{-} 0583$ & -909.297413 & 2.437 & 0.004251604 & -909.4286565 & 3.89 & 0.1713 \\
\hline $6 b_{-}^{-}$confs_0097 & -909.297347 & 2.478 & 0.003967343 & -909.42876 & 3.825 & 0.17098 \\
\hline $5 \mathrm{~b}^{-}$confs ${ }^{-} 0138$ & -909.297329 & 2.489 & 0.003894366 & -909.4283992 & 4.052 & 0.17103 \\
\hline 5a_confs_0601 & -909.297324 & 2.492 & 0.003874697 & -909.4287319 & 3.843 & 0.1715 \\
\hline $5 b^{-}$confs_-0090 & -909.29728 & 2.52 & 0.003695845 & -909.429684 & 3.246 & 0.1718 \\
\hline $5 a^{-}$confs -0789 & -909.297216 & 2.56 & 0.003454568 & -909.4285903 & 3.932 & 0.1712 \\
\hline $6 \mathrm{~b}^{-}$confs 0056 & -909.297202 & 2.569 & 0.003402489 & -909.4294034 & 3.422 & 0.1714 \\
\hline $6 \mathrm{~b}^{-} \operatorname{confs}{ }^{-} 0143$ & -909.297179 & 2.583 & 0.003323033 & -909.429643 & 3.271 & 0.1717 \\
\hline 5a_confs_ol12 & -909.297107 & 2.629 & 0.003074798 & -909.4275887 & 4.56 & 0.17112 \\
\hline 5b_confs_0070 & -909.297102 & 2.632 & 0.003059269 & -909.4282168 & 4.166 & 0.1710 \\
\hline $6 \mathrm{~b}^{-} \operatorname{confs}{ }^{-} 0687$ & -909.297064 & 2.656 & 0.002937823 & -909.4296585 & 3.262 & 0.1717 \\
\hline 6a_confs_0060 & -909.296925 & 2.743 & 0.002536615 & -909.4288476 & 3.77 & 0.1715 \\
\hline 6b_confs_0577 & -909.296864 & 2.781 & 0.002379033 & -909.4288802 & 3.75 & 0.17151 \\
\hline 5b_confs_0216 & -909.29676 & 2.846 & 0.002131844 & -909.4278895 & 4.372 & 0.1712 \\
\hline 5b_confs_0372 & -909.29673 & 2.865 & 0.002064564 & -909.4296091 & 3.292 & 0.1723 \\
\hline $6 a^{-} \operatorname{confs}{ }^{-} 0014$ & -909.29671 & 2.878 & 0.002019758 & -909.4292714 & 3.504 & 0.17169 \\
\hline 5a_confs_-0211 & -909.296683 & 2.895 & 0.00196263 & -909.4272432 & 4.777 & 0.17129 \\
\hline
\end{tabular}




\begin{tabular}{|c|c|c|c|c|c|c|}
\hline 5a_confs_0007 & -909.296658 & 2.91 & 0.001913566 & -909.4288515 & 3.768 & 0.172208 \\
\hline 5b_confs_o792 & -909.296589 & 2.954 & 0.001776607 & -909.4279065 & 4.361 & 0.17132 \\
\hline 5b_confs_0804 & -909.296588 & 2.954 & 0.001776607 & -909.4279065 & 4.361 & 0.1713 \\
\hline 5b_confs_0054 & -909.296582 & 2.958 & 0.001764653 & -909.4276029 & 4.551 & 0.1713 \\
\hline $5 b^{-}$confs 0525 & -909.29658 & 2.959 & 0.001761677 & -909.4276027 & 4.552 & 0.1713 \\
\hline a_confs_0049 & -909.296574 & 2.963 & 0.001749824 & -909.4292238 & 3.534 & 0.171 \\
\hline $\mathrm{b}^{-} \operatorname{confs}{ }^{-} 0124$ & -909.296566 & 2.968 & 0.001735119 & -909.4279494 & 4.334 & 0.171 \\
\hline 5a_confs_0297 & -909.296555 & 2.975 & 0.00171474 & -909.4287342 & 3.842 & 0.171 \\
\hline 5b_confs_0053 & -909.296508 & 3.004 & 0.001632831 & -909.4276464 & 4.524 & 0.1711 \\
\hline 6b_confs_0047 & -909.296477 & 3.024 & 0.001578633 & -909.4293829 & 3.434 & 0.171 \\
\hline 5a_confs_0438 & -909.296438 & 3.048 & 0.001515965 & -909.4277541 & 4.457 & 0.171 \\
\hline 5a_confs_0025 & -909.296365 & 3.094 & 0.001402721 & -909.4288781 & 3.751 & 0.171 \\
\hline $5 a^{-}$confs_0040 & -909.296324 & 3.12 & 0.001342497 & -909.4275169 & 4.605 & 0.171 \\
\hline 5b_confs_0042 & -909.296297 & 3.137 & 0.001304524 & -909.4273535 & 4.708 & 0.1710 \\
\hline 6a_confs_1150 & -909.296277 & 3.149 & 0.001278369 & -909.4289793 & 3.688 & 0.171 \\
\hline 5a_confs_0094 & -909.296213 & 3.19 & 0.001192897 & -909.4292188 & 3.537 & 0.1719 \\
\hline bb_confs_0942 & -909.29619 & 3.204 & 0.001165041 & -909.4281534 & 4.206 & 0.171 \\
\hline $6 a^{-}$confs 1062 & -909.296162 & 3.222 & 0.001130179 & -909.4287126 & 3.855 & 0.171 \\
\hline 6b_confs_1018 & -909.296145 & 3.232 & 0.001111264 & -909.4280063 & 4.298 & 0.1713 \\
\hline 5b_confs_0115 & -909.296061 & 3.285 & 0.001016174 & -909.4272787 & 4.755 & 0.11 \\
\hline bb_confs_0022 & -909.296015 & 3.314 & 0.000967634 & -909.4287234 & 3.848 & 0.1717 \\
\hline 5a_confs_0440 & -909.295956 & 3.351 & 0.000909055 & -909.4281649 & 4.199 & 0.1723 \\
\hline $5 b^{-}$confs_-0373 & -909.295893 & 3.39 & 0.000851144 & -909.4292126 & 3.541 & 0.1726 \\
\hline 6b_confs_0063 & -909.29586 & 3.411 & 0.000821504 & -909.4276252 & 4.537 & 0.171 \\
\hline 6a_confs_0043 & -909.295835 & 3.427 & 0.000799617 & -909.4288117 & 3.793 & $0.171 \mathrm{~s}$ \\
\hline 6b_confs_0023 & -909.295811 & 3.442 & 0.000779627 & -909.4277702 & 4.446 & 0.171 \\
\hline bb_confs_0110 & -909.295735 & 3.49 & 0.000718957 & -909.427072 & 4.885 & 0.1712 \\
\hline bb_confs_0093 & -909.295717 & 3.501 & 0.000705732 & -909.4274594 & 4.641 & 0.1716 \\
\hline 5b_confs_1155 & -909.295699 & 3.512 & 0.00069275 & -909.4271079 & 4.862 & 0.1715 \\
\hline 5b_confs_0059 & -909.295653 & 3.541 & 0.000659659 & -909.4272095 & 4.798 & 0.1715 \\
\hline 5a_confs_0783 & -909.295644 & 3.547 & 0.000653013 & -909.4280863 & 4.248 & 0.1724 \\
\hline b_confs_0015 & -909.295479 & 3.65 & 0.000548811 & -909.4278061 & 4.424 & 0.1714 \\
\hline A_confs_0021 & -909.295419 & 3.688 & 0.000514717 & -909.4282718 & 4.132 & 0.172 \\
\hline bb_confs_0082 & -909.295218 & 3.814 & 0.000416112 & -909.4277113 & 4.483 & 0.171 \\
\hline confs_0105 & -909.295074 & 3.904 & 0.000357471 & -909.4269769 & & 0.171 \\
\hline
\end{tabular}

a Hartrees. b kcal/mol. " Normalized based on Boltzmann distribution at 298.15 using Gibbs free energy window of $<4 \mathrm{kcal} / \mathrm{mol}$. 
M06-2X/6-31+G(d,p) Cartesian Coordinates (IEFPCM Implicit DMSO Solvent Model) for Systems A-D Within Free Energy Window of $4 \mathrm{kcal} / \mathrm{mol}$. ("6a" = species A; “6b" = species B; “5a" = species C; “5b" = species D).

\begin{tabular}{|c|c|c|c|}
\hline \multicolumn{4}{|c|}{ 6a_confs_0106 } \\
\hline 0 & -2.299210504745 & 5.806600435728 & 0.161284005414 \\
\hline $\mathrm{C}$ & -3.430735872498 & 4.985699102717 & 0.373356504107 \\
\hline $\mathrm{C}$ & -3.617376272356 & 3.939374527833 & -0.722848296696 \\
\hline $\mathrm{C}$ & -2.328148498520 & 3.124493366588 & -0.811982716083 \\
\hline $\mathrm{C}$ & -1.128091278765 & 4.048580176838 & -1.008322805714 \\
\hline $\mathrm{C}$ & -1.078251613507 & 5.094137995983 & 0.104491987981 \\
\hline $\mathrm{C}$ & -4.606477986251 & 5.941405867401 & 0.501404721944 \\
\hline 0 & -3.887673003659 & 4.583533961306 & -1.948852950732 \\
\hline 0 & -2.467453715534 & 2.251350234560 & -1.915922488722 \\
\hline 0 & 0.069919719231 & 3.302479098102 & -1.071635866286 \\
\hline 0 & -0.816586445107 & 4.400522845090 & 1.291951622005 \\
\hline $\mathrm{H}$ & -3.354517424901 & 4.464059383088 & 1.336852734208 \\
\hline $\mathrm{H}$ & -4.445693804023 & 3.275680443948 & -0.439706785176 \\
\hline $\mathrm{H}$ & -2.201029941362 & 2.553459134467 & 0.119172044790 \\
\hline $\mathrm{H}$ & -1.231364318643 & 4.566099605082 & -1.966228943253 \\
\hline $\mathrm{H}$ & -0.315890832579 & 5.850943445739 & -0.095929623605 \\
\hline $\mathrm{F}$ & -4.694007914592 & 6.816457602399 & -0.505836404009 \\
\hline $\mathrm{F}$ & -4.515118811163 & 6.659678543190 & 1.635960132830 \\
\hline $\mathrm{F}$ & -5.764575444159 & 5.260529332504 & 0.553184481825 \\
\hline $\mathrm{H}$ & -3.781901147642 & 3.919778871046 & -2.646336273896 \\
\hline $\mathrm{H}$ & -1.593311187798 & 1.891481596369 & -2.123460222948 \\
\hline $\mathrm{H}$ & 0.278899203726 & 3.001887173411 & -0.174126247787 \\
\hline $\mathrm{H}$ & -0.517102905150 & 5.014367256606 & 1.975031389802 \\
\hline \multicolumn{4}{|c|}{ 5a_confs_0182 } \\
\hline 0 & -4.992440903623 & 4.948641708832 & -1.232526576234 \\
\hline $\mathrm{C}$ & -6.182257340364 & 4.146260909941 & -1.246434856954 \\
\hline $\mathrm{C}$ & -5.884294615108 & 2.909607879833 & -0.390709753723 \\
\hline $\mathrm{C}$ & -4.352655553823 & 2.905873997288 & -0.328785237836 \\
\hline $\mathrm{C}$ & -4.040390330423 & 4.402861571067 & -0.349853232796 \\
\hline $\mathrm{C}$ & -7.353959953220 & 4.959312963023 & -0.700321083263 \\
\hline $\mathrm{C}$ & -7.716030204453 & 6.136522549301 & -1.601886810149 \\
\hline O & -7.128681421893 & 5.432674986399 & 0.598396615540 \\
\hline $\mathrm{H}$ & -6.386756310822 & 3.835399440542 & -2.276189367711 \\
\hline O & -6.453544759853 & 1.776863115140 & -1.006703716318 \\
\hline O & -3.808604583541 & 2.219216295455 & 0.766169744176 \\
\hline O & -4.237343738214 & 4.885816883576 & 0.961579122767 \\
\hline $\mathrm{H}$ & -6.281979197937 & 3.039275875147 & 0.625146457301 \\
\hline $\mathrm{H}$ & -3.965734458226 & 2.440147565398 & -1.240546631141 \\
\hline $\mathrm{H}$ & -3.048133729733 & 4.665189351980 & -0.718841264786 \\
\hline $\mathrm{H}$ & -8.238979480965 & 4.311683190072 & -0.682545932682 \\
\hline $\mathrm{F}$ & -6.775392605033 & 7.092515459960 & -1.613839072684 \\
\hline $\mathrm{F}$ & -8.857962204418 & 6.718888722021 & -1.193656737255 \\
\hline $\mathrm{F}$ & -7.905992173979 & 5.741399812326 & -2.874657008747 \\
\hline $\mathrm{H}$ & -6.181091623646 & 5.626830474809 & 0.707959536726 \\
\hline $\mathrm{H}$ & -6.418054764393 & 1.034247251763 & -0.390627450720 \\
\hline $\mathrm{H}$ & -3.940674420250 & 2.770831491883 & 1.552125462964 \\
\hline $\mathrm{H}$ & -3.681145626084 & 5.658438504242 & 1.124947793524 \\
\hline \multicolumn{4}{|c|}{ 6b_confs_0059 } \\
\hline 0 & -2.568790672451 & 5.541370608780 & 0.44759710567 \\
\hline
\end{tabular}




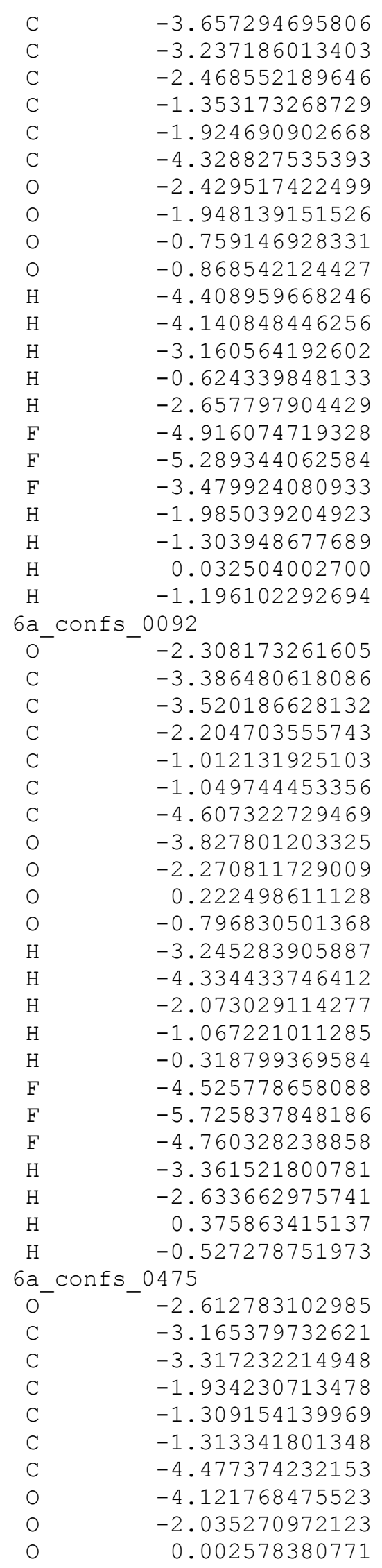

5.117112730916

4.174325392565

3.005850459476

3. 510939114964

4.453289481402

6.388535772528

4.874501019888

2.246657863166

2. 372151745486

4.951121506417

4.599834166141

3.787528012287

2.397917539655

4.077526094544

3.931917198765

7.044742636936

6.097285361914

7.245547006448

4.210754909668

1.620306410321

2.647135136240

5.341449831490

5.801777781229

4.937226515112

3.857024338730

3.066910230563

4.016067180032

5.143295019486

5.834767570142

4.393360761038

2. 116779857247

3. 341212297477

4. 552258183468

4.464799700343

3.172220362842

2.507421895977

4.467776452939

5.920248287144

6.619895561453

5.101287751341

6.645564546602

5.233211239585

2. 567264574027

3.090972621861

5.222557271362

5.609862346305

5.437812514111

3.955534577996

3. 299460132771

3.563870054891

5.063774429833

6.200304963316

3. 246117281318

1. 919289836632

3.056522093618
$-0.340365814020$

$-1.468911339088$

$-0.850757230263$

0.052080879930

1.103671666006

$-0.838290061824$

$-2.388260309491$

$-1.923188752310$

0.639069896198

1.844292787400

0.276232388004

$-1.958952407176$

$-0.248660535942$

$-0.543902966793$

1. 740189588327

0.179428600227

$-1.731807456709$

$-1.413927045936$

$-2.936402194869$

$-1.563853715689$

1.119990774400

2. 664926143942

0.055855957667

0.364029802392

$-0.704678344994$

$-0.774241861615$

$-0.959799264063$

0.077997100226

0.482882983793

$-1.981334095858$

$-1.813719611211$

$-0.899094409698$

1. 318784615767

1.344160149129

$-0.451087514219$

0.159422106475

$-1.956766980664$

$-0.157681981503$

1.569599794696

0.598233116904

$-0.574664751132$

$-2.105799671926$

$-2.591483544239$

0.024011645556

1.960074758517

$-1.204030436977$

0.083416349873

0.441370074020

0.365155785326

$-1.011895052389$

$-1.325897701861$

0.080395892178

$-0.484244574428$

0.632237651515

$-1.107326203151$ 


\begin{tabular}{|c|c|}
\hline & \\
\hline O & -0.416394993233 \\
\hline $\mathrm{H}$ & -2.536994928309 \\
\hline $\mathrm{H}$ & -3.706943582604 \\
\hline $\mathrm{H}$ & -1.289774577396 \\
\hline $\mathrm{H}$ & -1.904979292164 \\
\hline $\mathrm{H}$ & -1.022410028958 \\
\hline $\mathrm{F}$ & -5.342950398693 \\
\hline $\mathrm{F}$ & -4.285319142849 \\
\hline $\mathrm{F}$ & -5.082254605743 \\
\hline $\mathrm{H}$ & -5.057748351110 \\
\hline $\mathrm{H}$ & -2.766052802587 \\
\hline $\mathrm{H}$ & 0.577047456885 \\
\hline $\mathrm{H}$ & -0.170267748862 \\
\hline $6 a_{-}$ & 0103 \\
\hline 0 & -2.233968731564 \\
\hline $\mathrm{C}$ & -3.352814866710 \\
\hline C & -3.503099538612 \\
\hline $\mathrm{C}$ & -2.180972590192 \\
\hline $\mathrm{C}$ & -1.016540073451 \\
\hline $\mathrm{C}$ & -1.000131542454 \\
\hline $\mathrm{C}$ & -4.549353131440 \\
\hline 0 & -3.806947340882 \\
\hline 0 & -2.188995004694 \\
\hline 0 & 0.217375350574 \\
\hline 0 & -0.794213337656 \\
\hline $\mathrm{H}$ & -3.280840119600 \\
\hline $\mathrm{H}$ & -4.306394779896 \\
\hline $\mathrm{H}$ & -2.023285238696 \\
\hline $\mathrm{H}$ & -1.171936782846 \\
\hline $\mathrm{H}$ & -0.238686660236 \\
\hline $\mathrm{F}$ & -4.490836298488 \\
\hline $\mathrm{F}$ & -5.694816035692 \\
\hline $\mathrm{F}$ & -4.639442685639 \\
\hline $\mathrm{H}$ & -3.656045674933 \\
\hline $\mathrm{H}$ & -2.589280598653 \\
\hline $\mathrm{H}$ & 0.157628457462 \\
\hline $\mathrm{H}$ & -0.586002775703 \\
\hline $5 \mathrm{~b}$ & 1170 \\
\hline 0 & -6.159861361233 \\
\hline $\mathrm{C}$ & -5.910474040414 \\
\hline $\mathrm{C}$ & -5.487274549584 \\
\hline $\mathrm{C}$ & -5.820316213816 \\
\hline $\mathrm{C}$ & -5.569857152487 \\
\hline $\mathrm{C}$ & -7.175425510536 \\
\hline $\mathrm{C}$ & -7.590758212360 \\
\hline 0 & -8.271366058089 \\
\hline $\mathrm{H}$ & -5.088755389100 \\
\hline 0 & -4.124778167844 \\
\hline 0 & -7.201658686322 \\
\hline 0 & -4.175415900307 \\
\hline $\mathrm{H}$ & -6.059345826552 \\
\hline $\mathrm{H}$ & -5.190433381527 \\
\hline $\mathrm{H}$ & -6.049222435034 \\
\hline $\mathrm{H}$ & -6.951193847210 \\
\hline $\mathrm{F}$ & -8.048358848374 \\
\hline $\mathrm{F}$ & -8.575424020740 \\
\hline $\mathrm{F}$ & -6.563450226857 \\
\hline
\end{tabular}

5.656986508816

5.919349818636

3.858011831580

3.726186566593

3.052183927550

5.246915912052

5.754206453722

7.506169111072

6.091509989258

3. 344356288660

1. 578418163952

3.626203825822

6.537953371495

5.782700224569

4.941305933974

3.889060824631

3.117457301831

4.085873149922

5.079050307884

5.872767046164

4.522382193531

2.304669250406

3.406697919837

4.375388734067

4.421586623167

3.196482352302

2.513090448922

4.665190235391

5.848257673897

6.592175708957

5.168869552275

6.747427777041

3.869550177800

1.448468859619

2.745300816573

4.997446887242

4.231416620821

3.801978972335

2. 308774100296

1. 962463455342

3.304629226557

4.025264134544

5.494938814164

3.265250079209

4.381450999764

2.115337677647

1.697494350138

3.468354076435

1.683022835330

1.159750553480

3.426769164383

3.764915648683

5.940357029864

5.691638911027

6.287742417546
$-0.427593837710$

0.845435114289

1. 461551004569

1.141706043088

$-1.774099178369$

$-2.363111997255$

$-0.843874415911$

$-0.157882423192$

1.276858656142

$-0.272161028324$

0.094790977032

$-0.574758895111$

$-0.738041803352$

0.183118902572

0.378610917372

$-0.718174306792$

$-0.827995635589$

$-0.992410856115$

0.165087837799

0.488552692580

$-1.942175320635$

$-1.992404676720$

$-1.030997620275$

1. 351372085821

1.343284001493

$-0.429926629715$

0.072971200200

$-1.912446036893$

0.011788679252

1. 625070467859

0.522367943469

$-0.518950461751$

$-2.642077748968$

$-1.795271747115$

$-1.734239552879$

2.060545865031

0.380205725800

$-0.965764670055$

$-0.875839302959$

0.589951127866

1.262249844376

$-1.787640211716$

$-1.816296508868$

$-1.358556609941$

$-1.400036154154$

$-1.181082129952$

0.758786211860

1. 326129243971

$-1.565374573663$

0.981391044134

2.236017361425

$-2.829497090230$

$-0.638390169048$

$-2.713481886834$

$-2.177791022192$ 


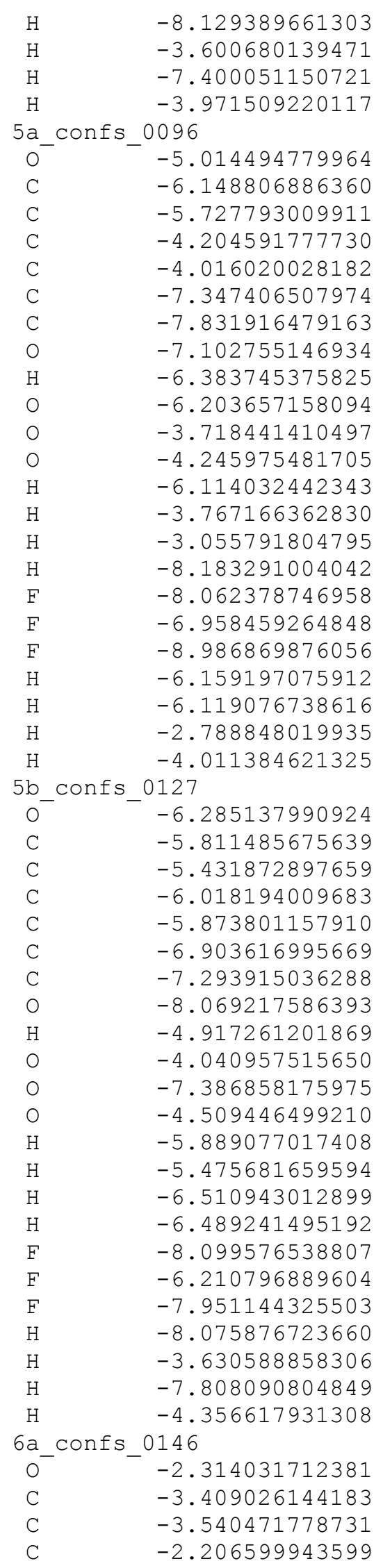

2.977054404572

2. 598291553804

0.808707216321

4.340397757739

5.039949615109

4.169065347877

3.010485608979

3.138885343974

4. 652723768229

4.938753300404

6.020262629144

5.519218214305

3.779736878658

1.799996586405

2.647082989643

5.301388608434

3.159064573813

2. 615881341320

4.965021094034

4.235264810476

5.518456580617

7.028868119562

6.560206352737

5.758340515525

1.104342927857

2.400015383692

4.685189409208

3.740042812860

3.927331048207

2. 515809287141

1.589091312784

2.469853508265

4.608261699496

5.965514421000

3.845923399283

4.559883033987

2. 355642077625

1. 402986516750

2.455168392387

2.307466395280

0.643062228901

2.199101952612

4.825947550809

6.630196248754

6.740178361702

5.877456877912

3.116103672765

2. 451058268563

0.850237530128

3.049583402789

5.851707800227

4.972200085416

3.968661481598

3.235244006844
$-0.438963348401$

$-0.523092307615$

0.435626570111

1.690448856087

$-1.384938066907$

$-1.278742390513$

$-0.366089867151$

$-0.323605555956$

$-0.478812488473$

$-0.724935315710$

$-1.687017618954$

0.524867386919

$-2.274528565700$

$-0.907553536987$

0.906024593354

0.755415053747

0.650969843862

$-1.180926086159$

$-0.895592845380$

$-0.623211221240$

$-2.914915631726$

$-1.822364394892$

$-1.257751786978$

0.593761617715

$-0.243417532144$

0.821049946251

1. 469314463021

0.442471953622

$-0.897929894308$

$-1.428474796346$

$-0.350969791878$

0.888433631018

$-1.716113842590$

$-1.134921910991$

$-1.866300107994$

$-0.895948495921$

$-1.583230159752$

$-0.662472617811$

1.227859380563

$-2.398444635156$

$-0.278536587877$

1. 734774878128

$-2.708824653553$

$-1.984862563487$

$-0.930979925970$

0.029149017279

$-1.220747057494$

$-0.709520563445$

0.008917614260

1.974971129709

0.216603613546

0.377817969842

$-0.766040925403$

$-0.909308029046$ 


\begin{tabular}{|c|c|}
\hline & \\
\hline $\mathrm{C}$ & -1.069559357784 \\
\hline $\mathrm{C}$ & -1.060760691598 \\
\hline $\mathrm{C}$ & -4.628873917473 \\
\hline 0 & -3.864598360689 \\
\hline 0 & -2.303901137638 \\
\hline 0 & 0.122563529593 \\
\hline 0 & -0.820708397182 \\
\hline $\mathrm{H}$ & -3.317673858485 \\
\hline $\mathrm{H}$ & -4.323882956810 \\
\hline $\mathrm{H}$ & -2.033133612576 \\
\hline $\mathrm{H}$ & -1.246937531134 \\
\hline $\mathrm{H}$ & -0.324940811601 \\
\hline $\mathrm{F}$ & -4.746451265337 \\
\hline $\mathrm{F}$ & -4.582700961957 \\
\hline F & -5.755669476319 \\
\hline $\mathrm{H}$ & -3.711863144226 \\
\hline $\mathrm{H}$ & -1.409189978651 \\
\hline $\mathrm{H}$ & 0.793204028459 \\
\hline $\mathrm{H}$ & -0.621392519700 \\
\hline & 0242 \\
\hline 0 & -6.201710199974 \\
\hline $\mathrm{C}$ & -5.829399909797 \\
\hline $\mathrm{C}$ & -5.463533359470 \\
\hline $\mathrm{C}$ & -5.949403437039 \\
\hline $\mathrm{C}$ & -5.731091730190 \\
\hline $\mathrm{C}$ & -6.992456360764 \\
\hline $\mathrm{C}$ & -7.352276100491 \\
\hline 0 & -8.124505450412 \\
\hline $\mathrm{H}$ & -4.952617569277 \\
\hline 0 & -4.081475291413 \\
\hline 0 & -7.304213858578 \\
\hline 0 & -4.344422307260 \\
\hline $\mathrm{H}$ & -5.982109423626 \\
\hline $\mathrm{H}$ & -5.356740990708 \\
\hline $\mathrm{H}$ & -6.302060321115 \\
\hline $\mathrm{H}$ & -6.688360339007 \\
\hline $\mathrm{F}$ & -6.277894515032 \\
\hline $\mathrm{F}$ & -7.854099166583 \\
\hline $\mathrm{F}$ & -8.281963130035 \\
\hline $\mathrm{H}$ & -8.684530261383 \\
\hline $\mathrm{H}$ & -3.614410406608 \\
\hline $\mathrm{H}$ & -7.812952468161 \\
\hline $\mathrm{H}$ & -4.156773403080 \\
\hline & 0067 \\
\hline 0 & -2.280829721497 \\
\hline $\mathrm{C}$ & -3.362177451102 \\
\hline $\mathrm{C}$ & -3.437390324114 \\
\hline $\mathrm{C}$ & -2.088696310100 \\
\hline $\mathrm{C}$ & -0.958778343707 \\
\hline $\mathrm{C}$ & -1.013727772974 \\
\hline $\mathrm{C}$ & -4.602817921827 \\
\hline 0 & -3.630538360977 \\
\hline 0 & -2.044197367280 \\
\hline 0 & 0.300014673196 \\
\hline 0 & -0.807806341368 \\
\hline $\mathrm{H}$ & -3.285590801754 \\
\hline $\mathrm{H}$ & -4.228119701594 \\
\hline
\end{tabular}

4.238855534299

5.183117188086

5.865838757924

4.651632693685

2. 430514362952

3. 490621318571

4.434239058801

4.410313706919

3.242793828234

2. 615362115454

4.847407940196

5.982802112880

6.784342879719

6.532162460455

5.131841463822

4.032700099015

2.142723754093

4.016714723599

5.028902627210

3. 865845455001

3. 918772319726

2. 465205241275

1. 642348058933

2. 650893615462

4.485548324551

5.912578089427

3.666140113477

4.562079085747

2. 314923872521

1.275213836701

2.702393263452

2.153967026893

0.735283416552

2.456080897322

4.534198565598

6.718645911364

6.002837468424

6.414764217952

3.678991929288

2. 490994521084

2.038022874462

3. 387271894789

5.813008229821

4.919639858247

3. 873957918722

3.153511752134

4.175332655429

5.166883544734

5.789508517146

4.450975087006

2.246598653306

3.542400991667

4. 473028010515

4.390509035192

3.150451497082
$-1.048872935993$

0.155889709396

0.536148757162

$-1.957886349887$

$-2.068601092099$

$-1.175075972508$

1.306556311125

1. 316857074516

$-0.509452909322$

$-0.019310478185$

$-1.943595772003$

0.030919610531

$-0.428465421445$

1. 705082232763

0.541346738506

$-2.687300070565$

$-2.300403004453$

$-1.627293463999$

2. 041284407522

0.448731210017

$-0.933003537424$

$-1.345019298755$

$-0.134488893722$

0.990443395629

$-1.735593722372$

$-1.331142067125$

$-1.553746801697$

$-1.066551766042$

$-1.588574085857$

$-0.230000027602$

1.229412609055

$-2.254974595603$

$-0.003608241564$

1. 901115952841

$-2.787804552857$

$-1.395806007440$

$-0.094934023699$

$-2.167660207895$

$-2.340562473062$

$-0.756938600271$

$-0.554758934948$

1.885464670391

0.134939605707

0.301482255538

$-0.819103399800$

$-0.880609740143$

$-1.008580686552$

0.149510517621

0.395654687619

$-2.099378042232$

$-1.963215685423$

$-1.015002500055$

1.340788783796

1. 261357105123

$-0.590709609736$ 


\begin{tabular}{|c|c|c|c|}
\hline & & & \\
\hline $\mathrm{H}$ & -1.940006784689 & 2.568622032587 & 0.032522744768 \\
\hline $\mathrm{H}$ & -1.108311361342 & 4.752603348637 & -1.931962720507 \\
\hline $\mathrm{H}$ & -0.286479290399 & 5.972235767546 & 0.016842287635 \\
\hline $\mathrm{F}$ & -4.584799483167 & 6.549635251047 & 1.502131374803 \\
\hline $\mathrm{F}$ & -5.708093258158 & 5.023185932596 & 0.451065067287 \\
\hline $\mathrm{F}$ & -4.745137571930 & 6.618377828366 & -0.648215462445 \\
\hline $\mathrm{H}$ & -4.571459221275 & 4.528911634414 & -2.297809156973 \\
\hline $\mathrm{H}$ & -2.426220027056 & 2.700855990001 & -2.730418954363 \\
\hline $\mathrm{H}$ & 0.261711337877 & 2.838016030416 & -1.677310802570 \\
\hline $\mathrm{H}$ & -0.647248594760 & 5.103050433394 & 2.055322330901 \\
\hline $6 a_{-}$ & 0093 & & \\
\hline 0 & -2.358230137443 & 5.818233059267 & 0.186450682000 \\
\hline C & -3.489874035747 & 4.987433918812 & 0.356072692664 \\
\hline $\mathrm{C}$ & -3.636354355884 & 3.959232395485 & -0.762402848845 \\
\hline $\mathrm{C}$ & -2.345393604729 & 3.146898201613 & -0.820131913205 \\
\hline $\mathrm{C}$ & -1.140677827566 & 4.069335112213 & -0.954197101796 \\
\hline C & -1.137939813258 & 5.122406902255 & 0.161939314432 \\
\hline $\mathrm{C}$ & -4.674158318563 & 5.935335132482 & 0.461870729790 \\
\hline 0 & -3.866242794864 & 4.623914924968 & -1.985770174941 \\
\hline 0 & -2.449052294924 & 2.290139675037 & -1.939905716256 \\
\hline 0 & 0.007770107225 & 3.241882912454 & -0.850164126065 \\
\hline 0 & -0.939301648591 & 4.517518791992 & 1.414015444812 \\
\hline $\mathrm{H}$ & -3.436714447713 & 4.453291150458 & 1.313607700289 \\
\hline $\mathrm{H}$ & -4.471248681957 & 3.288353965151 & -0.519164286821 \\
\hline $\mathrm{H}$ & -2.248385418897 & 2.562967256695 & 0.105858674421 \\
\hline $\mathrm{H}$ & -1.180864216461 & 4.578264701391 & -1.923527331469 \\
\hline $\mathrm{H}$ & -0.378121659263 & 5.888487206120 & -0.027059481121 \\
\hline $\mathrm{F}$ & -4.621496560190 & 6.637312500651 & 1.608695989622 \\
\hline $\mathrm{F}$ & -5.829156981197 & 5.247540863734 & 0.467932486375 \\
\hline $\mathrm{F}$ & -4.735702390822 & 6.824855097330 & -0.534841516540 \\
\hline $\mathrm{H}$ & -3.746865753588 & 3.971273341795 & -2.691311804876 \\
\hline $\mathrm{H}$ & -1.586260158565 & 1.876428212922 & -2.082949177271 \\
\hline $\mathrm{H}$ & 0.757402306677 & 3.656101257853 & -1.296312157229 \\
\hline $\mathrm{H}$ & -0.284231313679 & 3.811393419321 & 1.301593922030 \\
\hline $6 a_{-}$ & 0079 & & \\
\hline 0 & -2.301639555990 & 5.883539358372 & 0.175482368807 \\
\hline $\mathrm{C}$ & -3.361389631369 & 4.959969682921 & 0.347798570501 \\
\hline $\mathrm{C}$ & -3.402552258987 & 3.956510324632 & -0.804210537813 \\
\hline $\mathrm{C}$ & -2.039313716148 & 3.257804619128 & -0.876096140884 \\
\hline C & -0.911141071486 & 4.287959601496 & -0.942905960090 \\
\hline $\mathrm{C}$ & -1.015194201914 & 5.265923310881 & 0.224376158334 \\
\hline C & -4.621324443009 & 5.801768667370 & 0.464315164822 \\
\hline 0 & -3.705397293239 & 4.560779130518 & -2.050848150968 \\
\hline 0 & -1.971281897422 & 2.398512874019 & -1.996162900777 \\
\hline 0 & 0.351841134169 & 3.667450401306 & -0.917897363941 \\
\hline 0 & -0.829720866080 & 4.567504352703 & 1.412564252947 \\
\hline $\mathrm{H}$ & -3.257199101876 & 4.415248410673 & 1.294438514078 \\
\hline $\mathrm{H}$ & -4.182904290966 & 3.211471003309 & -0.625097940600 \\
\hline $\mathrm{H}$ & -1.897114642840 & 2.634543069676 & 0.013132303994 \\
\hline $\mathrm{H}$ & -1.023658164444 & 4.880460405763 & -1.863773857359 \\
\hline $\mathrm{H}$ & -0.305835567114 & 6.090424920750 & 0.117470320229 \\
\hline $\mathrm{F}$ & -5.714956618315 & 5.023053057688 & 0.455985983639 \\
\hline $\mathrm{F}$ & -4.746591718867 & 6.688592485915 & -0.534509174916 \\
\hline $\mathrm{F}$ & -4.631976748079 & 6.500374846793 & 1.611308497577 \\
\hline $\mathrm{H}$ & -3.278070291857 & 5.427836185000 & -2.111205432861 \\
\hline $\mathrm{H}$ & -2.360476499537 & 2.872195745027 & -2.747870995729 \\
\hline $\mathrm{H}$ & 0.348215306486 & 2.980705019219 & -1.599614828807 \\
\hline
\end{tabular}




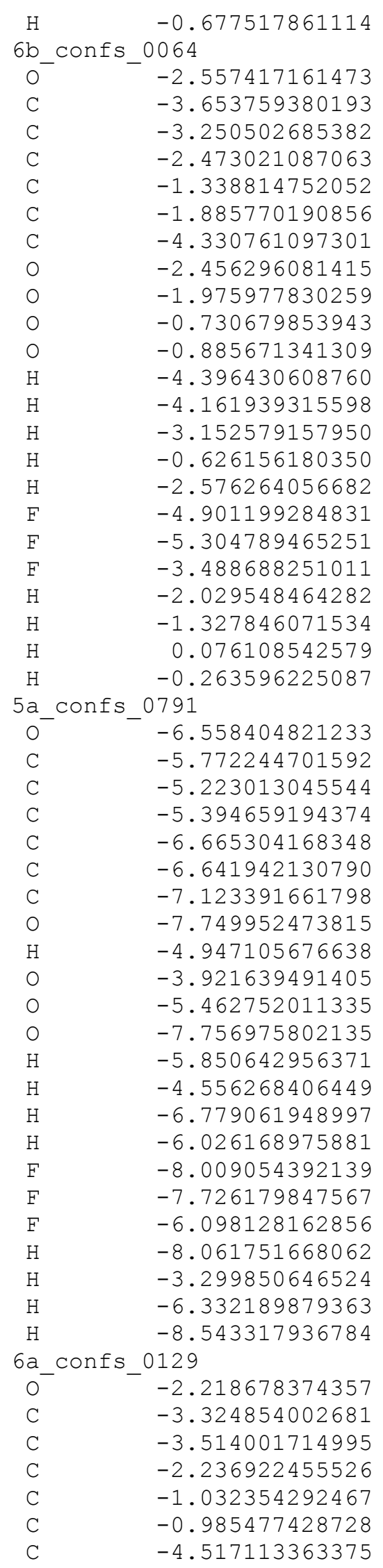

5.190672526840

5.536343377747

5.103731152484

4.151659572310

2. 990532967802

3.510017067879

4.464848306504

6.372012844749

4.843231212219

2.214161580013

2.377756247009

4.998522617803

4.592519565273

3.761063411210

2.393476533950

4.064248773256

3.938912652272

7.038506302079

6.073753649799

7.221654769407

4.174927536546

1.589994447518

2.648736860454

5. 487988551718

3.526331638374

4.031754198544

2.801762693397

1. 679013858180

2.124066010641

4.909740839488

6.172884861070

4.227111541906

4.623635789352

2.971633060959

0.386621502149

1.750229820483

2.578423219430

1.698754286770

1.736058760346

5.252934222258

5.917960384125

7.007939120766

6.847800373833

3. 578688055727

3.089892470307

0.288439972372

1. 596323319520

5.755401899064

4.895188386483

3.961480011533

3.130863793226

4.060463827605

5.071040946848

5.795226058980
2.135021149816

0.441104587913

$-0.332644567509$

$-1.458650889661$

$-0.840248233471$

0.031108652863

1.091451407553

$-0.831907299623$

$-2.396193025797$

$-1.911165428376$

0.616230287746

1.886520814358

0.299011224278

$-1.930912168346$

$-0.213668817921$

$-0.597806621784$

1.765830462002

0.188277945918

$-1.708103684124$

$-1.428002710171$

$-2.952750657056$

$-1.555014751467$

1.073348760067

1.327684712612

0.679964680230

$-0.408803716955$

$-1.157315709798$

$-0.126068476336$

0.600352594079

$-1.306473842748$

$-0.597562483566$

$-1.823572840592$

0.002597611005

$-1.662040145207$

$-0.661689950711$

$-0.211662873730$

$-2.026790478129$

0.579199069960

1. 613199670013

$-2.146843865859$

0.376780475069

$-1.462941194430$

$-0.044729843556$

$-1.167757085201$

$-0.931167484095$

$-1.078405344636$

0.327731235189

0.182529397851

0.362768650719

$-0.846161171096$

$-0.930031043551$

$-1.085656967918$

0.063159131508

0.627353207049 


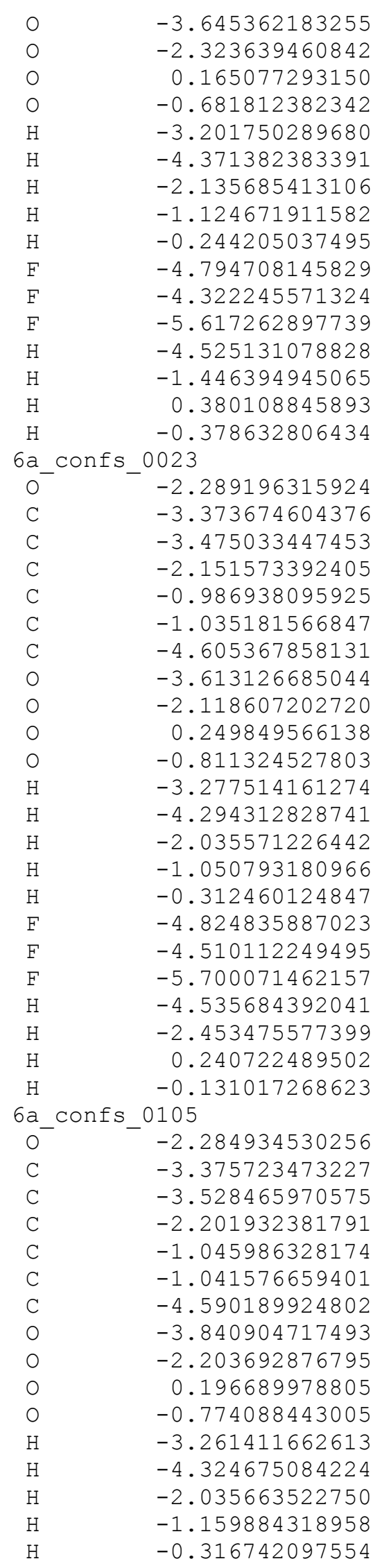

4.670314799107

2.238424825559

3. 312752822764

4. 341821476686

4.287232692811

3.298911533323

2. 572163792435

4.602352815881

5.851398924173

6.615733134549

6.568160386736

5.055734189921

5.060805622485

1.846966756341

3.020723069504

4.937938233987

5.872804456302

4.985015218025

3.966150748105

3.204687436538

4.187992843953

5.237418356203

5.860478290978

4.567953342907

2.281472254273

3.515158594397

4.645176891163

4.438998607348

3.265801945032

2.623581116848

4.707218795379

6.037150931206

6.632319136198

6.677853692858

5.098364894148

4.786594427759

2.739807694411

2.749529135947

3.963171190025

5.829815190698

4.954521871515

3.929344532458

3.173689875004

4.150200706684

5.182970740991

5.854512690255

4.590256911547

2.356187688843

3.478516765049

4.569296559516

4.415348839906

3.224012837312

2. 572044982583

4.679563287000

5.976234844431
$-2.059116798656$

$-2.017294617399$

$-1.153038672504$

1.220035338275

1.269456678837

$-0.672470793537$

0.013066314513

$-2.030431550255$

$-0.124903685459$

$-0.401181957866$

1. 704840013367

0.851495830320

$-2.123692511063$

$-2.135350527946$

$-0.254091954202$

1. 917017689009

0.221972597647

0.399686918920

$-0.744045257278$

$-0.789989476667$

$-0.912360819425$

0.200170458683

0.539253439274

$-2.017799850971$

$-1.858182290313$

$-0.777083945917$

1.454024081363

1. 347791122299

$-0.543392020350$

0.130767774317

$-1.877654737632$

0.010339928176

$-0.537088028825$

1. 597759277125

0.712148964075

$-2.196819922513$

$-2.644518416979$

$-1.368991768888$

1.341411973877

0.144172795356

0.353131519918

$-0.767847425260$

$-0.894423003188$

$-1.064954767371$

0.063603188688

0.517898771028

$-1.974162785367$

$-2.054268507497$

$-0.998350861125$

1.298994018611

1.302515486555

$-0.491007826751$

0.007764236444

$-2.019332702223$

$-0.144743090847$ 


\begin{tabular}{|c|c|c|c|}
\hline $\mathrm{F}$ & -4.515285885159 & 6.551434240166 & 1.666554195275 \\
\hline $\mathrm{F}$ & -5.715820160729 & 5.121261736631 & 0.568884376338 \\
\hline F & -4.730145205450 & 6.746372198318 & -0.468761732877 \\
\hline $\mathrm{H}$ & -3.708658233792 & 3.952080285422 & -2.690901486706 \\
\hline $\mathrm{H}$ & -2.608934085371 & 1.502245695357 & -1.856243100361 \\
\hline $\mathrm{H}$ & 0.167848452198 & 2.732274165012 & -1.613418881804 \\
\hline $\mathrm{H}$ & -0.088622868885 & 3.898813355302 & 1.154097583164 \\
\hline \multicolumn{4}{|c|}{ 6a_confs_0051 } \\
\hline 0 & -2.303795633486 & 5.893972505795 & 0.143244624768 \\
\hline C & -3.340630734629 & 4.950628063936 & 0.352679036227 \\
\hline C & -3.400426049089 & 3.953819818385 & -0.803693204488 \\
\hline C & -2.039576265399 & 3.257147461959 & -0.889382247487 \\
\hline C & -0.910139914271 & 4.284030491790 & -0.984047410871 \\
\hline C & -1.014454066219 & 5.322806751978 & 0.135265686434 \\
\hline C & -4.608310319711 & 5.774209275210 & 0.510566638853 \\
\hline 0 & -3.714686388119 & 4.565019551451 & -2.043198138161 \\
\hline 0 & -1.982530898398 & 2.375741815172 & -1.991619958180 \\
\hline 0 & 0.350447388874 & 3.661541690226 & -0.842552294770 \\
\hline 0 & -0.775898563529 & 4.737862037165 & 1.386576464890 \\
\hline $\mathrm{H}$ & -3.193068102393 & 4.408680765774 & 1.294604121032 \\
\hline $\mathrm{H}$ & -4.177884015906 & 3.207422439028 & -0.617794174139 \\
\hline $\mathrm{H}$ & -1.889541836818 & 2.647011663267 & 0.008269916687 \\
\hline $\mathrm{H}$ & -0.973786173443 & 4.811302181131 & -1.946917821197 \\
\hline $\mathrm{H}$ & -0.333866427502 & 6.160502142655 & -0.045485872079 \\
\hline $\mathrm{F}$ & -5.688588566592 & 4.977840079079 & 0.541455407607 \\
\hline $\mathrm{F}$ & -4.780688582412 & 6.654525289913 & -0.486871243684 \\
\hline $\mathrm{F}$ & -4.590499569316 & 6.476215239221 & 1.654703031764 \\
\hline $\mathrm{H}$ & -3.297836772694 & 5.437701718480 & -2.096592058333 \\
\hline $\mathrm{H}$ & -2.359218151614 & 2.840591597485 & -2.754985522354 \\
\hline $\mathrm{H}$ & 0.383324482715 & 2.905581114969 & -1.445966484794 \\
\hline $\mathrm{H}$ & -0.066044840048 & 4.085646305931 & 1.279141502275 \\
\hline \multicolumn{4}{|c|}{ 6a_confs_0097 } \\
\hline 0 & -2.298240265289 & 5.811400339869 & 0.115461436641 \\
\hline C & -3.383151799442 & 4.924325723049 & 0.333149421043 \\
\hline C & -3.500900031788 & 3.941192448568 & -0.834518743487 \\
\hline C & -2.188703013350 & 3.152526951791 & -0.867524350442 \\
\hline C & -0.995921730946 & 4.101419303928 & -0.995382740146 \\
\hline C & -1.034574357166 & 5.162995839285 & 0.106733875922 \\
\hline C & -4.603649708899 & 5.812248608110 & 0.511390684711 \\
\hline 0 & -3.772803832462 & 4.606327319293 & -2.049839663271 \\
\hline 0 & -2.221389559991 & 2.246382935517 & -1.946221444316 \\
\hline 0 & 0.219343213828 & 3.383200258340 & -0.972350642876 \\
\hline 0 & -0.779971840775 & 4.501033493234 & 1.310466012185 \\
\hline $\mathrm{H}$ & -3.256451636661 & 4.374544967038 & 1.273900165699 \\
\hline $\mathrm{H}$ & -4.330880489069 & 3.249308570795 & -0.664363140614 \\
\hline $\mathrm{H}$ & -2.103055009216 & 2.608344802064 & 0.086280801471 \\
\hline $\mathrm{H}$ & -1.039516025290 & 4.602153439693 & -1.968407462857 \\
\hline $\mathrm{H}$ & -0.309679552038 & 5.958807266868 & -0.079332052915 \\
\hline $\mathrm{F}$ & -4.735863415364 & 6.725396285137 & -0.460928445877 \\
\hline $\mathrm{F}$ & -4.542458856249 & 6.485722993411 & 1.672977564547 \\
\hline $\mathrm{F}$ & -5.725693158799 & 5.074684817100 & 0.533034770709 \\
\hline $\mathrm{H}$ & -3.238153883166 & 5.410942215267 & -2.106747318662 \\
\hline $\mathrm{H}$ & -1.344316869639 & 1.842919909757 & -2.011412992153 \\
\hline $\mathrm{H}$ & 0.390876309917 & 3.124645529017 & -0.053913699921 \\
\hline $\mathrm{H}$ & -0.516544488147 & 5.134475982867 & 1.990647964612 \\
\hline \multicolumn{4}{|c|}{ 5a_confs_0814 } \\
\hline 0 & & 3.131411930087 & -1.2 \\
\hline
\end{tabular}




\begin{tabular}{|c|c|}
\hline \multirow{2}{*}{\multicolumn{2}{|c|}{-6.865927721767}} \\
\hline & \\
\hline $\mathrm{C}$ & -5.914683691035 \\
\hline $\mathrm{C}$ & -4.577034470523 \\
\hline $\mathrm{C}$ & -5.028935969533 \\
\hline $\mathrm{C}$ & -7.296952630796 \\
\hline $\mathrm{C}$ & -8.234260259624 \\
\hline 0 & -6.226331668447 \\
\hline $\mathrm{H}$ & -7.751440517288 \\
\hline 0 & -6.395406243503 \\
\hline 0 & -3.926772477524 \\
\hline O & -4.088602175145 \\
\hline $\mathrm{H}$ & -5.847264588449 \\
\hline $\mathrm{H}$ & -3.921643716096 \\
\hline $\mathrm{H}$ & -5.339328820459 \\
\hline $\mathrm{H}$ & -7.884956105247 \\
\hline $\mathrm{F}$ & -9.273286857025 \\
\hline $\mathrm{F}$ & -7.632179892656 \\
\hline $\mathrm{F}$ & -8.747447077362 \\
\hline $\mathrm{H}$ & -5.432690647924 \\
\hline $\mathrm{H}$ & -5.898780846301 \\
\hline $\mathrm{H}$ & -3.434626079044 \\
\hline $\mathrm{H}$ & -3.647271755926 \\
\hline \multicolumn{2}{|c|}{$5 a$ confs 0511} \\
\hline 0 & -5.676472529238 \\
\hline $\mathrm{C}$ & -5.905947882074 \\
\hline $\mathrm{C}$ & -5.208349314808 \\
\hline $\mathrm{C}$ & -4.319857731578 \\
\hline $\mathrm{C}$ & -5.124423736245 \\
\hline $\mathrm{C}$ & -7.408821879951 \\
\hline $\mathrm{C}$ & -8.082740044067 \\
\hline 0 & -8.081287813864 \\
\hline $\mathrm{H}$ & -5.438970185523 \\
\hline 0 & -4.409747378493 \\
\hline 0 & -4.112573575144 \\
\hline 0 & -6.179548468951 \\
\hline $\mathrm{H}$ & -5.941546940714 \\
\hline $\mathrm{H}$ & -3.372651726789 \\
\hline $\mathrm{H}$ & -4.538977258655 \\
\hline $\mathrm{H}$ & -7.553973251409 \\
\hline $\mathrm{F}$ & -7.440141238998 \\
\hline $\mathrm{F}$ & -8.142441139620 \\
\hline $\mathrm{F}$ & -9.348611875522 \\
\hline $\mathrm{H}$ & -7.646602238498 \\
\hline $\mathrm{H}$ & -4.946395704481 \\
\hline $\mathrm{H}$ & -3.667157270103 \\
\hline $\mathrm{H}$ & -5.886760815277 \\
\hline \multicolumn{2}{|c|}{ 5b_confs_0170 } \\
\hline 0 & -6.289779819301 \\
\hline $\mathrm{C}$ & -5.784790182124 \\
\hline $\mathrm{C}$ & -5.349001008169 \\
\hline $\mathrm{C}$ & -5.978360776261 \\
\hline C & -5.897131563735 \\
\hline $\mathrm{C}$ & -6.866264340532 \\
\hline $\mathrm{C}$ & -7.292342063423 \\
\hline 0 & -8.013781143662 \\
\hline $\mathrm{H}$ & -4.905131819300 \\
\hline 0 & -3.931076417083 \\
\hline
\end{tabular}

3.379190080141

3.007200606534

2.793641516886

2.292541963051

4.842145653039

5.192344038805

5.745810137246

2.736098797728

1. 801005800360

4.028214471276

2.430013242370

3. 802065765239

2.090009872740

1.243303317139

5.001417427440

4.336924622249

5.182646865325

6.425650267488

5.303633543836

1.602579769822

4. 321713063331

3.284437247867

4.680794195494

4.010008980824

2.637309825659

2.807595493106

3.793350212652

3.888833888477

5.252408843910

3.150454837882

4.597097007834

2. 312988821277

1.629516068826

3.127162395564

1.846109167579

3.271838316355

4.379496221867

3.389016568417

6.033264023012

5.925911524530

5.116818959986

3.285858012176

1.874390127620

0.972421041663

2.226355465292

3.687124569644

3.902618731400

2.516830074985

1.557914645555

2. 401600813228

4.567777036542

5.915508318528

3.786831142867

4.555228796029

2.474667606666
$-0.049430721424$

1. 114276057347

0.398010466863

$-0.976412411086$

$-0.018334750270$

$-1.171652808834$

$-0.028779120015$

0.013561109569

1.674396831278

0.133640105338

$-1.972952321997$

1.865840861915

0.917920314450

$-0.946316981180$

0.894156587826

$-1.234252608159$

$-2.368175737456$

$-1.004791804757$

$-0.376436711920$

2. 478629751323

0.910726845588

$-1.842132630877$

0.207209830137

$-1.042236736950$

$-0.934037920605$

0.297991427500

1.139345456426

$-1.296018975486$

$-1.434976856848$

$-0.315210465316$

$-1.838837229270$

$-2.050751870710$

1.039932262492

1.807131316234

$-0.735323375462$

$-0.004722197028$

1.850695152768

$-2.261949474119$

$-2.324302395496$

$-0.277374270632$

$-1.871508044681$

0.548525242760

$-2.721933474321$

0.489376562370

2.021976036236

0.343229251047

$-0.977413112225$

$-1.504215536917$

$-0.477328417226$

0.794402995309

$-1.823585209701$

$-1.243757068582$

$-2.007836951494$

$-0.945233976905$

$-1.418366007128$ 


\begin{tabular}{|c|c|c|c|}
\hline & & & \\
\hline 0 & -7.357309847174 & 1.361884807421 & -0.736223809608 \\
\hline O & -4.624267741527 & 2.398797971388 & 1.365338867599 \\
\hline $\mathrm{H}$ & -5.696702467817 & 2.322309867433 & -2.524202746139 \\
\hline $\mathrm{H}$ & -5.437519103157 & 0.610969217703 & -0.394161455141 \\
\hline $\mathrm{H}$ & -6.607501661521 & 2.097369229891 & 1.563650165268 \\
\hline $\mathrm{H}$ & -6.432058537646 & 4.797326102283 & -2.804650329194 \\
\hline $\mathrm{F}$ & -6.228104860446 & 6.705463013444 & -1.005301002632 \\
\hline $\mathrm{F}$ & -7.979849416213 & 5.807912990454 & -0.099192663459 \\
\hline $\mathrm{F}$ & -8.084945953766 & 6.572625146854 & -2.111593764823 \\
\hline $\mathrm{H}$ & -8.044965521724 & 3.085774851671 & -1.332073911077 \\
\hline $\mathrm{H}$ & -3.605452017690 & 1.667047271986 & -1.837892371577 \\
\hline $\mathrm{H}$ & -7.464005598176 & 0.741442093331 & -1.469513355314 \\
\hline $\mathrm{H}$ & -3.958458139553 & 2.476475700699 & 0.662120409918 \\
\hline $6 b_{3}$ & 0141 & & \\
\hline 0 & -2.424433502622 & 5.561771907156 & 0.359655811381 \\
\hline C & -3.554500779247 & 5.061150965558 & -0.321712856720 \\
\hline C & -3.116611344011 & 4.133741219584 & -1.454166261215 \\
\hline $\mathrm{C}$ & -2.284517867070 & 2.982251226940 & -0.866367959111 \\
\hline $\mathrm{C}$ & -1.172740181066 & 3.519475681197 & 0.034716132597 \\
\hline $\mathrm{C}$ & -1.733708628028 & 4.516983364162 & 1.045077224694 \\
\hline $\mathrm{C}$ & -4.331717178686 & 6.279826774680 & -0.796544894340 \\
\hline 0 & -2.364690198926 & 4.816663700045 & -2.442710366996 \\
\hline 0 & -1.770656933922 & 2.182976630109 & -1.906534232347 \\
\hline 0 & -0.581682329291 & 2.430025684233 & 0.704785687576 \\
\hline 0 & -0.663196835341 & 5.069135759741 & 1.722678813434 \\
\hline $\mathrm{H}$ & -4.222069461425 & 4.513539023419 & 0.360504176761 \\
\hline $\mathrm{H}$ & -3.990790370520 & 3.716772621806 & -1.961463054643 \\
\hline $\mathrm{H}$ & -2.933703261767 & 2.337150256622 & -0.262139859361 \\
\hline $\mathrm{H}$ & -0.427069417156 & 4.053363406902 & -0.575848935669 \\
\hline $\mathrm{H}$ & -2.437568591997 & 4.019043225630 & 1.730237211564 \\
\hline $\mathrm{F}$ & -3.563314815395 & 7.159177963976 & -1.454794244952 \\
\hline $\mathrm{F}$ & -4.877624311732 & 6.935557545716 & 0.240464471149 \\
\hline $\mathrm{F}$ & -5.330465778873 & 5.915247788897 & -1.615221563258 \\
\hline $\mathrm{H}$ & -1.771764283166 & 5.454813673890 & -2.018028698916 \\
\hline $\mathrm{H}$ & -1.424038781006 & 2.782714909101 & -2.584708862381 \\
\hline $\mathrm{H}$ & 0.136873331951 & 2.769807562060 & 1.255289861211 \\
\hline $\mathrm{H}$ & -0.967308480703 & 5.488009108577 & 2.538432399542 \\
\hline & 0109 & & \\
\hline 0 & -6.281975053985 & 3.681074491025 & 0.463826708883 \\
\hline $\mathrm{C}$ & -5.811067552436 & 3.855390094698 & -0.87469019702 \\
\hline C & -5.456112285172 & 2.443982899288 & -1.395119309849 \\
\hline C & -6.096340817883 & 1.527478452682 & -0.34423140829 \\
\hline $\mathrm{C}$ & -5.941438021227 & 2.383364290207 & 0.918591453610 \\
\hline $\mathrm{C}$ & -6.880144163408 & 4.562387155346 & -1.702847018291 \\
\hline $\mathrm{C}$ & -7.238957234329 & 5.926906487635 & -1.117581388063 \\
\hline 0 & -8.065518956523 & 3.836111590388 & -1.869870781106 \\
\hline $\mathrm{H}$ & -4.900662334398 & 4.465236719470 & -0.878624517151 \\
\hline 0 & -4.040880686815 & 2.337461880284 & -1.350319030107 \\
\hline 0 & -7.461975427466 & 1.389944380944 & -0.691832756135 \\
\hline 0 & -4.654095774556 & 2.319527797202 & 1.454762418583 \\
\hline $\mathrm{H}$ & -5.847029776091 & 2.247653280291 & -2.398400217320 \\
\hline $\mathrm{H}$ & -5.594295906794 & 0.558319768972 & -0.26910910032 \\
\hline $\mathrm{H}$ & -6.639595528595 & 2.129554242285 & 1.718276444295 \\
\hline $\mathrm{H}$ & -6.448683101269 & 4.774960715474 & -2.689494406769 \\
\hline $\mathrm{F}$ & -7.911115766335 & 5.850760784938 & 0.038321225241 \\
\hline $\mathrm{F}$ & -8.016746653497 & 6.616642641516 & -1.97345516323 \\
\hline $\mathrm{F}$ & -6.137659838697 & 6.670681567488 & -0.89850510268 \\
\hline
\end{tabular}




\begin{tabular}{|c|c|c|c|}
\hline & & & \\
\hline $\mathrm{H}$ & -8.093490507873 & 3.091856001426 & -1.241299825996 \\
\hline $\mathrm{H}$ & -3.765482972789 & 1.511153690540 & -1.769425864557 \\
\hline $\mathrm{H}$ & -7.917853364279 & 0.848574649293 & -0.033943764874 \\
\hline $\mathrm{H}$ & -4.005478275582 & 2.356676418606 & 0.732371601168 \\
\hline $5 a$ & 0079 & & \\
\hline 0 & -5.007571803491 & 5.025329036832 & -1.343085404277 \\
\hline $\mathrm{C}$ & -6.133339760855 & 4.140432341805 & -1.270657564793 \\
\hline $\mathrm{C}$ & -5.686135832984 & 2.931125932244 & -0.425465373999 \\
\hline $\mathrm{C}$ & -4.163431704113 & 3.079765522429 & -0.385601233290 \\
\hline $\mathrm{C}$ & -3.998621801233 & 4.602716138182 & -0.464809993633 \\
\hline $\mathrm{C}$ & -7.331453855888 & 4.864303401570 & -0.655958554656 \\
\hline $\mathrm{C}$ & -7.828220109581 & 6.008135336669 & -1.536652458853 \\
\hline O & -7.080660273520 & 5.357404710673 & 0.629405381216 \\
\hline $\mathrm{H}$ & -6.383046922335 & 3.819306860611 & -2.287707201445 \\
\hline O & -6.162612552318 & 1.693700143391 & -0.891913724272 \\
\hline O & -3.660013672479 & 2.537883453543 & 0.813975746780 \\
\hline O & -4.228554130205 & 5.181573582549 & 0.803216328792 \\
\hline $\mathrm{H}$ & -6.061289622533 & 3.024513357631 & 0.598912317530 \\
\hline $\mathrm{H}$ & -3.710277773864 & 2.613739854644 & -1.268733287935 \\
\hline $\mathrm{H}$ & -3.045073472726 & 4.948332037395 & -0.870344327632 \\
\hline $\mathrm{H}$ & -8.163199945179 & 4.151063659774 & -0.596827829699 \\
\hline $\mathrm{F}$ & -8.057298808250 & 5.594740163427 & -2.797370363971 \\
\hline $\mathrm{F}$ & -6.964115486569 & 7.031672267225 & -1.599788835492 \\
\hline $\mathrm{F}$ & -8.986822853890 & 6.505236421961 & -1.067309867149 \\
\hline $\mathrm{H}$ & -6.140069935064 & 5.606500430120 & 0.705727243754 \\
\hline $\mathrm{H}$ & -5.822928591832 & 1.523969452938 & -1.781044953045 \\
\hline $\mathrm{H}$ & -2.729648888135 & 2.302347209110 & 0.707823790009 \\
\hline $\mathrm{H}$ & -3.971012202956 & 4.535008685276 & 1.481610166060 \\
\hline $5 b$ & 0112 & & \\
\hline 0 & -6.338063193115 & 3.665453619544 & 0.464124088196 \\
\hline $\mathrm{C}$ & -5.844506879095 & 3.937495582795 & -0.855182255152 \\
\hline $\mathrm{C}$ & -5.344362931061 & 2.581185053762 & -1.423380148096 \\
\hline $\mathrm{C}$ & -5.947413850874 & 1.558855447672 & -0.443434850165 \\
\hline $\mathrm{C}$ & -5.927108506581 & 2.367966658302 & 0.852685905709 \\
\hline $\mathrm{C}$ & -7.003350769666 & 4.550039930102 & -1.651937430004 \\
\hline $\mathrm{C}$ & -7.230109549707 & 6.019984734855 & -1.313372100964 \\
\hline O & -8.201929809473 & 3.843153329227 & -1.438754614549 \\
\hline $\mathrm{H}$ & -5.002519344539 & 4.636514943545 & -0.813898017837 \\
\hline O & -3.935358459362 & 2.519394654617 & -1.472403578212 \\
\hline O & -7.253072708904 & 1.166408908573 & -0.794816810971 \\
\hline O & -4.594690675158 & 2.377610536466 & 1.300096566965 \\
\hline $\mathrm{H}$ & -5.702325094075 & 2.402403892131 & -2.440449900824 \\
\hline $\mathrm{H}$ & -5.330418440322 & 0.661449634397 & -0.372638300850 \\
\hline $\mathrm{H}$ & -6.622391215770 & 2.023701137087 & 1.621696948504 \\
\hline $\mathrm{H}$ & -6.777881687903 & 4.511965132227 & -2.721449503150 \\
\hline $\mathrm{F}$ & -7.636495340602 & 6.180680102392 & -0.041074513234 \\
\hline $\mathrm{F}$ & -8.174572335787 & 6.560713309830 & -2.098025487031 \\
\hline $\mathrm{F}$ & -6.109076676891 & 6.740364761079 & -1.474961160486 \\
\hline $\mathrm{H}$ & -8.390606454682 & 3.880896807281 & -0.485690276321 \\
\hline $\mathrm{H}$ & -3.603764343226 & 2.558401641266 & -0.561377832392 \\
\hline $\mathrm{H}$ & -7.740678503843 & 1.928355667315 & -1.148431751620 \\
\hline $\mathrm{H}$ & -4.523103229366 & 2.929204515538 & 2.090975022480 \\
\hline & 0114 & & \\
\hline 0 & -6.258751490535 & 3.654215968862 & 0.395991522211 \\
\hline $\mathrm{C}$ & -5.803535009586 & 3.882157218607 & -0.937545591902 \\
\hline $\mathrm{C}$ & -5.385593563324 & 2.503398938985 & -1.495507660851 \\
\hline $\mathrm{C}$ & -5.967043247326 & 1.524867584364 & -0.459533101451 \\
\hline
\end{tabular}




\begin{tabular}{|c|c|}
\hline & \\
\hline $\mathrm{C}$ & -5.846384241132 \\
\hline $\mathrm{C}$ & -6.913734737168 \\
\hline $\mathrm{C}$ & -7.305629116318 \\
\hline 0 & -8.052270690303 \\
\hline $\mathrm{H}$ & -4.926849293081 \\
\hline 0 & -3.963415653032 \\
\hline 0 & -7.303150601754 \\
\hline 0 & -4.552855931077 \\
\hline $\mathrm{H}$ & -5.774231691280 \\
\hline $\mathrm{H}$ & -5.385793257784 \\
\hline $\mathrm{H}$ & -6.527493151517 \\
\hline $\mathrm{H}$ & -6.539051360330 \\
\hline $\mathrm{F}$ & -8.187036545247 \\
\hline $\mathrm{F}$ & -6.234764522655 \\
\hline F & -7.880902900789 \\
\hline $\mathrm{H}$ & -8.562703316245 \\
\hline $\mathrm{H}$ & -3.652194807220 \\
\hline $\mathrm{H}$ & -7.794241701339 \\
\hline $\mathrm{H}$ & -3.913373170959 \\
\hline & 0448 \\
\hline 0 & -2.200373841839 \\
\hline $\mathrm{C}$ & -3.465957965433 \\
\hline $\mathrm{C}$ & -3.377518043757 \\
\hline $\mathrm{C}$ & -2.423523008826 \\
\hline $\mathrm{C}$ & -1.097918889958 \\
\hline $\mathrm{C}$ & -1.347950579120 \\
\hline $\mathrm{C}$ & -4.330568366512 \\
\hline 0 & -2.824478129335 \\
\hline 0 & -2.244125800239 \\
\hline 0 & -0.316641715756 \\
\hline 0 & -0.125807357086 \\
\hline $\mathrm{H}$ & -3.946113534435 \\
\hline $\mathrm{H}$ & -4.369028417032 \\
\hline $\mathrm{H}$ & -2.872227255344 \\
\hline $\mathrm{H}$ & -0.587056304063 \\
\hline $\mathrm{H}$ & -1.824563720715 \\
\hline $\mathrm{F}$ & -4.520542345717 \\
\hline $\mathrm{F}$ & -5.540943791380 \\
\hline $\mathrm{F}$ & -3.801566912852 \\
\hline $\mathrm{H}$ & -3.512050274445 \\
\hline $\mathrm{H}$ & -2.080190119156 \\
\hline $\mathrm{H}$ & 0.517310573733 \\
\hline $\mathrm{H}$ & -0.185164200731 \\
\hline & 0204 \\
\hline 0 & -6.340850423594 \\
\hline $\mathrm{C}$ & -5.784271926414 \\
\hline $\mathrm{C}$ & -5.313936542775 \\
\hline $\mathrm{C}$ & -5.994863408710 \\
\hline $\mathrm{C}$ & -5.979041814957 \\
\hline $\mathrm{C}$ & -6.832560664223 \\
\hline $\mathrm{C}$ & -7.286760878360 \\
\hline 0 & -7.970331482656 \\
\hline $\mathrm{H}$ & -4.918013157594 \\
\hline 0 & -3.918103128918 \\
\hline 0 & -7.358245515000 \\
\hline 0 & -4.741104300364 \\
\hline $\mathrm{H}$ & -5.60504828800 \\
\hline
\end{tabular}

2.362578725869

4.540789129710

5.910087989172

3. 711157665272

4.540284414167

2. 475857779239

1.177521892125

2. 368022407700

2. 313685164065

0.601571912906

2.050375421518

4.715523803213

6.507205513091

6.717265899603

5.853424288594

3.828399334776

1.661398668558

1.981165200318

2. 447545079283

5.917567144722

5.308252710691

3.885920371613

3.061953136839

3. 801101431588

5.220558377050

6.238921124925

3. 856267001135

1.780056704208

3.090818154821

5.868715641439

5.257687074507

3.419784137906

2. 917957388522

3. 876918964831

5.195371409407

7. 413940726046

5.690344316885

6.506469109090

3.979601994378

1.901883706563

3.566844527792

6.652064845042

3.691006256635

3.915296469952

2. 528056931631

1. 566726254427

2. 398383772266

4.577318456136

5.923519190907

3.795565801895

4.583161704833

2.354252980478

1. 371840372837

2. 382612550381

2.336008664353
0.818986245348

$-1.745991171366$

$-1.197000679048$

$-1.742072852947$

$-0.936508941116$

$-1.470511903516$

$-0.728793006559$

1. 349256948313

$-2.501536232595$

$-0.401809689664$

1. 611385730599

$-2.761418800077$

$-2.023251736325$

$-1.101825818848$

0.008557103135

$-2.553683678849$

$-1.887585815751$

$-0.972376335673$

0.622475466931

$-0.154663910597$

$-0.237121709439$

$-0.805992329312$

0.068485656586

0.243443443513

0.746700781671

$-1.070610845255$

$-2.109311444329$

$-0.485959842992$

1.178542240003

0.786246019682

0.753599461794

$-0.786220736087$

1.058355220105

$-0.728069623225$

1.740259913616

$-0.451960598450$

$-1.278631295085$

$-2.273227498217$

$-2.774812965336$

$-1.433499285275$

1.291183289816

1.348266056813

0.390424996926

$-0.907767960466$

$-1.418578023856$

$-0.434231652816$

0.848155593941

$-1.798528916437$

$-1.237229829961$

$-2.032419258404$

$-0.831556509216$

$-1.228244959763$

$-0.763490231612$

1. 490441645178

$-2.456775410369$ 


\begin{tabular}{|c|c|}
\hline & \\
\hline $\mathrm{H}$ & -5.452952732110 \\
\hline $\mathrm{H}$ & -6.732924691068 \\
\hline $\mathrm{H}$ & -6.356607335554 \\
\hline $\mathrm{F}$ & -8.014586300777 \\
\hline $\mathrm{F}$ & -8.050244631809 \\
\hline $\mathrm{F}$ & -6.235535376032 \\
\hline $\mathrm{H}$ & -8.015580078517 \\
\hline $\mathrm{H}$ & -3.425531784510 \\
\hline $\mathrm{H}$ & -7.428068001402 \\
\hline $\mathrm{H}$ & -4.035137536647 \\
\hline & 0119 \\
\hline 0 & -2.526453963880 \\
\hline $\mathrm{C}$ & -3.635444764530 \\
\hline C & -3.219074955192 \\
\hline $\mathrm{C}$ & -2.429143236621 \\
\hline $\mathrm{C}$ & -1.289368901893 \\
\hline $\mathrm{C}$ & -1.836859712220 \\
\hline $\mathrm{C}$ & -4.362851835978 \\
\hline 0 & -2.345159484970 \\
\hline 0 & -1.915110318035 \\
\hline 0 & -0.656118428257 \\
\hline 0 & -0.783429735246 \\
\hline $\mathrm{H}$ & -4.348710869475 \\
\hline $\mathrm{H}$ & -4.112570839838 \\
\hline $\mathrm{H}$ & -3.098062396800 \\
\hline $\mathrm{H}$ & -0.573846880633 \\
\hline $\mathrm{H}$ & -2.544212483593 \\
\hline $\mathrm{F}$ & -5.400025114050 \\
\hline $\mathrm{F}$ & -3.577271949789 \\
\hline $\mathrm{F}$ & -4.850730979228 \\
\hline $\mathrm{H}$ & -2.844348386255 \\
\hline $\mathrm{H}$ & -1.538829685390 \\
\hline $\mathrm{H}$ & -0.462954864780 \\
\hline $\mathrm{H}$ & -1.121220213348 \\
\hline & 0053 \\
\hline 0 & -2.395086102197 \\
\hline $\mathrm{C}$ & -3.536594507239 \\
\hline $\mathrm{C}$ & -3.119483250839 \\
\hline $\mathrm{C}$ & -2.285843353984 \\
\hline $\mathrm{C}$ & -1.143607884351 \\
\hline $\mathrm{C}$ & -1.677550194078 \\
\hline $\mathrm{C}$ & -4.314437555098 \\
\hline 0 & -2.376961324430 \\
\hline 0 & -1.764863524682 \\
\hline 0 & -0.502718581385 \\
\hline 0 & -0.615082916687 \\
\hline $\mathrm{H}$ & -4.196906480421 \\
\hline $\mathrm{H}$ & -4.001511735473 \\
\hline $\mathrm{H}$ & -2.928830660110 \\
\hline $\mathrm{H}$ & -0.424240359448 \\
\hline $\mathrm{H}$ & -2.366496904342 \\
\hline $\mathrm{F}$ & -3.553448535726 \\
\hline $\mathrm{F}$ & -4.831051506604 \\
\hline $\mathrm{F}$ & -5.334264903318 \\
\hline $\mathrm{H}$ & -1.781974685838 \\
\hline $\mathrm{H}$ & -1.422250168927 \\
\hline $\mathrm{H}$ & -0.292023528422 \\
\hline
\end{tabular}

0.622704637679

2.088995977152

4.810146631677

5.814564076521

6.575580954414

6.719265655029

3.075858696626

2.918390578414

0.747510065876

2.454833319883

5.616774498930

5.111150068976

4.092425280768

2. 988595862256

3. 574849771574

4.607756187707

6.318479134291

4.635982592810

2.055336810135

2. 552719679689

5.210808670644

4.622661442875

3.661740835085

2. 431112994285

4.089285124008

4.140058194941

5.923174476368

7.088488444182

7.098648411954

5.051829098273

2. 561975936520

1.832751326484

5.723795157246

5.564016994899

5.037557726355

4.055435076819

2.952137269633

3.532850991345

4.568934246578

6.237148971251

4.677512486950

2.062393184553

2. 511774225199

5.189362060558

4.524982824815

3.612775996223

2. 356350914453

4.047155470324

4.106078236434

7.072956607534

6.951441307420

5.839478177878

5.341284842457

2. 597796746138

1.801041175222
$-0.338659065833$

1.572595900776

$-2.759666216847$

$-0.118119396631$

$-2.134491030270$

$-0.961710512308$

$-1.377075620663$

$-1.838401370825$

$-1.497862272805$

0.826990102260

0.425801479082

$-0.276124479703$

$-1.347539918264$

$-0.645875324033$

0.191249831900

1. 165033779693

$-0.842850473305$

$-2.318610351977$

$-1.571898176624$

0.928636022188

1.822380463866

0.407373362930

$-1.813775151770$

0.020316022387

$-0.467576146464$

1.869321168298

$-1.602681404833$

$-1.609726307912$

0.132930202017

$-3.032023776862$

$-2.308572030549$

0.311221414820

2.568189795117

0.390617704813

$-0.249718615087$

$-1.345051988533$

$-0.686465411535$

0.152322186526

1. 130789159186

$-0.769325472316$

$-2.378410679673$

$-1.651229930075$

0.881873322679

1. 752656371034

0.465929090869

$-1.816067363267$

$-0.027275860801$

$-0.505255641620$

1.854787169184

$-1.490340955507$

0.243329014644

$-1.545599119758$

$-1.998224745577$

$-2.383964046912$

0.259703299743 


\begin{tabular}{|c|c|}
\hline $\mathrm{H}$ & -0.936071336 \\
\hline \multicolumn{2}{|c|}{$5 b$ confs 0509} \\
\hline 0 & -5.277323962291 \\
\hline $\mathrm{C}$ & -6.534217943437 \\
\hline $\mathrm{C}$ & -6.819657446856 \\
\hline $\mathrm{C}$ & -5.800341831476 \\
\hline $\mathrm{C}$ & -4.631847047674 \\
\hline $\mathrm{C}$ & -7.602770119615 \\
\hline $\mathrm{C}$ & -7.250217439731 \\
\hline 0 & -7.873480620626 \\
\hline $\mathrm{H}$ & -6.458892544641 \\
\hline 0 & -6.475251158498 \\
\hline 0 & -6.356595192113 \\
\hline 0 & -3.871420246395 \\
\hline $\mathrm{H}$ & -7.845959355950 \\
\hline $\mathrm{H}$ & -5.539080794958 \\
\hline $\mathrm{H}$ & -3.946162379493 \\
\hline $\mathrm{H}$ & -8.524293660488 \\
\hline $\mathrm{F}$ & -6.214069126920 \\
\hline $\mathrm{F}$ & -8.294690702114 \\
\hline $\mathrm{F}$ & -6.951350151230 \\
\hline $\mathrm{H}$ & -7.185085518808 \\
\hline $\mathrm{H}$ & -7.098704638698 \\
\hline $\mathrm{H}$ & -5.728229301621 \\
\hline $\mathrm{H}$ & -4.465358816368 \\
\hline \multicolumn{2}{|c|}{$5 a$ confs 0093} \\
\hline 0 & -5.018200015794 \\
\hline $\mathrm{C}$ & -6.188877278603 \\
\hline $\mathrm{C}$ & -5.816627768261 \\
\hline $\mathrm{C}$ & -4.293160820559 \\
\hline $\mathrm{C}$ & -4.032967047581 \\
\hline $\mathrm{C}$ & -7.357686539343 \\
\hline $\mathrm{C}$ & -7.770765426156 \\
\hline 0 & -7.108428016463 \\
\hline $\mathrm{H}$ & -6.437671629973 \\
\hline 0 & -6.372352233707 \\
\hline 0 & -3.782619408455 \\
\hline 0 & -4.228542884861 \\
\hline $\mathrm{H}$ & -6.165169915562 \\
\hline $\mathrm{H}$ & -3.937077211922 \\
\hline $\mathrm{H}$ & -3.059295411143 \\
\hline $\mathrm{H}$ & -8.228178253061 \\
\hline $\mathrm{F}$ & -7.984014583403 \\
\hline $\mathrm{F}$ & -6.856030957013 \\
\hline $\mathrm{F}$ & -8.916799076583 \\
\hline $\mathrm{H}$ & -6.154806062357 \\
\hline $\mathrm{H}$ & -6.284407306255 \\
\hline $\mathrm{H}$ & -2.839339104299 \\
\hline $\mathrm{H}$ & -3.747783048647 \\
\hline \multicolumn{2}{|c|}{$5 a$ confs 0867} \\
\hline $0^{-}$ & -5.422240669041 \\
\hline $\mathrm{C}$ & -6.070162723212 \\
\hline $\mathrm{C}$ & -5.626785757631 \\
\hline $\mathrm{C}$ & -4.415311299551 \\
\hline $\mathrm{C}$ & -4.809087894838 \\
\hline $\mathrm{C}$ & -7.584928277835 \\
\hline $\mathrm{C}$ & -8.020509921444 \\
\hline
\end{tabular}

\begin{tabular}{lr}
5.704234466961 & \multicolumn{1}{l}{2.504722511981} \\
3.936096105690 & -0.626575927089 \\
3.284579750915 & -0.825058703549 \\
2.494780861913 & 0.479672529821 \\
3.084528465690 & 1.455812538496 \\
3.391376534830 & 0.511068170610 \\
4.321397482305 & -1.163622440599 \\
5.106383122442 & -2.425637230829 \\
5.241306893430 & -0.143561509927 \\
2.579694415978 & -1.661580301206 \\
1.125527015259 & 0.338025107979 \\
4.281584302035 & 1.967516478548 \\
2.260117149788 & 0.211341743423 \\
2.619106283415 & 0.839541710649 \\
2.379994841024 & 2.249674628769 \\
4.153088435133 & 0.886572440691 \\
3.777731403439 & -1.410182583269 \\
5.939085660547 & -2.262969874696 \\
5.859371458492 & -2.819746788232 \\
4.281373114263 & -3.447645703496 \\
5.191465437420 & 0.544617850797 \\
0.686550369380 & -0.254999597437 \\
4.707380030634 & 2.565326481066 \\
1.505480865978 & 0.064410979481
\end{tabular}

$-1.397034448148$

$-1.279599971267$

$-0.343653325482$

$-0.353056522272$

$-0.474399002000$

$-0.743335541157$

$-1.702156925535$

0.516912952730

$-2.267896801631$

$-0.840550867425$

0.790795218359

0.775891039429

0.679044564733

$-1.274810832584$

$-0.891181674911$

$-0.662004531033$

$-2.944647396905$

$-1.795795010744$

$-1.298162995067$

0.608483481904

$-0.167718068787$

0.677245206714

0.806531451080

0.017802932134

$-1.177642743460$

$-1.367093559722$

$-0.444373425803$

0.689134041554

$-1.034096136023$

$-0.862706869744$ 


\begin{tabular}{|c|c|}
\hline & \\
\hline O & -8.113513896748 \\
\hline $\mathrm{H}$ & -5.716152501677 \\
\hline 0 & -5.241197896842 \\
\hline 0 & -4.115703214135 \\
\hline 0 & -5.730536862724 \\
\hline $\mathrm{H}$ & -6.398047323733 \\
\hline $\mathrm{H}$ & -3.558509826965 \\
\hline $\mathrm{H}$ & -3.969355662465 \\
\hline $\mathrm{H}$ & -8.051322699440 \\
\hline $\mathrm{F}$ & -7.669136962818 \\
\hline $\mathrm{F}$ & -9.359003046216 \\
\hline $\mathrm{F}$ & -7.493775915872 \\
\hline $\mathrm{H}$ & -7.453513922478 \\
\hline $\mathrm{H}$ & -6.003403159103 \\
\hline $\mathrm{H}$ & -3.760160524686 \\
\hline $\mathrm{H}$ & -5.710640040547 \\
\hline $5 a_{-}$ & 0719 \\
\hline 0 & -6.758198989706 \\
\hline $\mathrm{C}$ & -6.659252012301 \\
\hline $\mathrm{C}$ & -5.198330063501 \\
\hline $\mathrm{C}$ & -4.489779991238 \\
\hline $\mathrm{C}$ & -5.599616742362 \\
\hline C & -7.691063736978 \\
\hline $\mathrm{C}$ & -7.878884281518 \\
\hline 0 & -8.952189404735 \\
\hline $\mathrm{H}$ & -6.919376698442 \\
\hline 0 & -4.746121448564 \\
\hline 0 & -3.308872644807 \\
\hline 0 & -5.650888033690 \\
\hline $\mathrm{H}$ & -5.092964488591 \\
\hline $\mathrm{H}$ & -4.237719089151 \\
\hline $\mathrm{H}$ & -5.496158340804 \\
\hline $\mathrm{H}$ & -7.346416567891 \\
\hline $\mathrm{F}$ & -6.752140549313 \\
\hline $\mathrm{F}$ & -8.211796811259 \\
\hline $\mathrm{F}$ & -8.847654290731 \\
\hline $\mathrm{H}$ & -8.815897462393 \\
\hline $\mathrm{H}$ & -3.893220096309 \\
\hline $\mathrm{H}$ & -3.561220632152 \\
\hline $\mathrm{H}$ & -6.193237623565 \\
\hline $5 a_{-}$ & 0251 \\
\hline 0 & -5.481899934076 \\
\hline $\mathrm{C}$ & -6.724960851015 \\
\hline $\mathrm{C}$ & -6.447403651645 \\
\hline $\mathrm{C}$ & -5.022071868779 \\
\hline $\mathrm{C}$ & -4.411329755891 \\
\hline C & -7.720570316862 \\
\hline $\mathrm{C}$ & -8.014966131621 \\
\hline 0 & -7.210495537413 \\
\hline $\mathrm{H}$ & -7.129196445799 \\
\hline 0 & -6.505359913063 \\
\hline 0 & -4.973434164291 \\
\hline 0 & -3.312984398063 \\
\hline $\mathrm{H}$ & -7.155335025954 \\
\hline $\mathrm{H}$ & -4.487243505890 \\
\hline $\mathrm{H}$ & -4.124240247251 \\
\hline $\mathrm{H}$ & -8.674884485847 \\
\hline
\end{tabular}

3. 356943155880

4. 574022550231

2.195773045158

1.128204410841

2.708267320861

1.815001056121

2.867652930554

3. 754071254009

3.775010596594

6.083748125045

5.678182646528

6.355974943627

3.291778540450

1.878405965130

0.614848667488

3.114891497767

4.272551241848

4.583479015940

4.346481464842

4.186948544117

3.575498773749

3.703911229431

4.112288350921

3. 832350136001

5.639681510759

5.441014051796

3. 431142823975

2.216351517582

3.409807095666

5.180034908241

3.717871110092

2.659839583287

3. 919510274901

5.408086921974

3.396213625914

3. 753858876514

5.217081561914

2.499506875600

1.728490504932

3.797369233049

3.198043032058

2.196814683355

2.555260304942

3. 048977661342

4.277870145205

5.264050324965

4.990210333628

2. 641304170097

0.902056467295

3.593780349684

3. 865104933016

2.303462278097

1. 696408890109

2. 226015472677

3. 791250669325
0.019271960055

$-2.021179684302$

$-2.688065190931$

0.016893931517

1.531917181197

$-1.010315410694$

$-0.965496250132$

1.260890597777

$-1.967542840996$

0.323682086618

$-0.953366778336$

$-1.819164476685$

0.733752471215

$-3.186532037719$

$-0.719837969686$

2. 408068172164

1.361244150834

$-0.038166626538$

$-0.440297707594$

0.914393563834

1.767595671139

$-0.754091747428$

$-2.205416882572$

$-0.149730573566$

$-0.163454844129$

$-1.206133421493$

0.872926354821

1. 424170005407

$-1.004675080098$

1.297909227108

2. 844860510898

$-0.752710310578$

$-2.912869641262$

$-2.328753175140$

$-2.795850799171$

0.806273714431

$-1.599210517573$

0.786801446460

2.057186682211

$-1.152074839011$

$-0.814745888803$

0.331555772072

0.759880486540

$-0.559385940852$

$-0.408989423810$

$-1.535760246010$

0.696102488292

$-1.668677954457$

$-0.235923003554$

1.716430791037

$-0.393135658878$

1.161990503004

1.170764886447

$-1.222288612113$

$-0.174135062198$ 


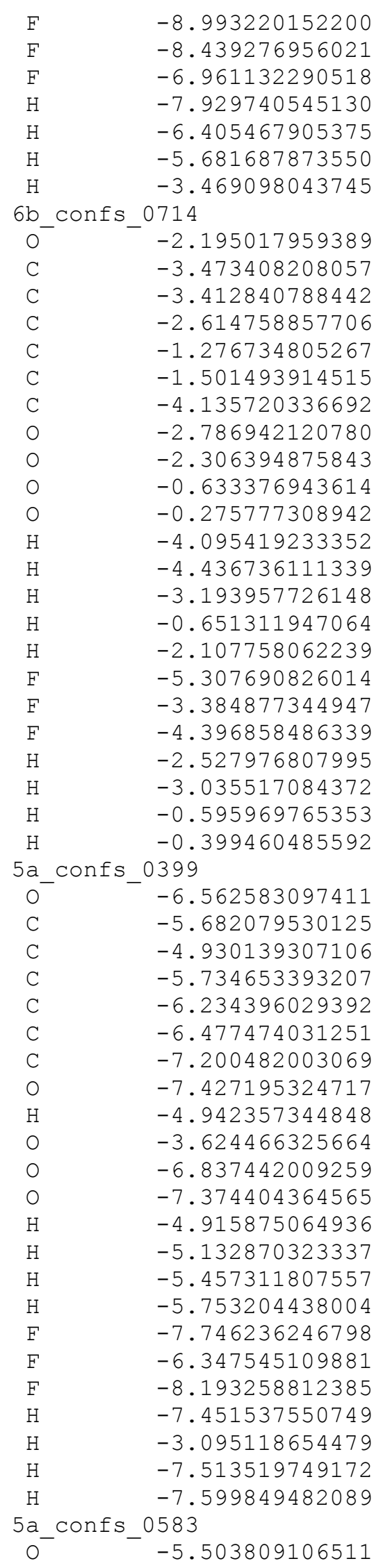

6.114782095298

4.621779797665

6.011163578186

5.341045485805

0.243876028190

4.234610974225

4.390763091792

5.383891085758

5.137578442951

4.352915806162

3.070600141858

3.365051799266

4.186858405627

6.500115237461

5.135420359568

2.427240153968

2.162620830004

4.544825966856

4.571252169279

4.088403661760

2.403813194362

3.962957117771

3.622885654752

6.390875392234

7.388783725217

7.029002915505

4.533066006132

1.851970894284

1.608266483187

4.901604556040

3.694511747910

4.060367901887

2.775278905356

1. 673118357464

2.386943232035

4.686366147491

5.956970754542

3.839593583026

4.786753676853

2.866958767055

1.358786435125

1.752061420616

2.634853846050

0.786731058004

2.448352886693

5.014921865725

6.578782761181

6.829005157051

5.736427603853

2.984584948631

2.126554502119

0.925112243866

2.072962197467

4.055036034123
$-1.166111887226$

$-2.638052433190$

$-1.879164113277$

1.237678617958

0.463880160546

1. 523399107333

0.408762250148

0.442169281540

$-0.092958362566$

$-1.402632531552$

$-1.142663217629$

$-0.471512780225$

0.789465165968

$-0.233326458352$

$-2.393694944584$

$-2.369287525517$

$-0.112648125020$

1. 314165224933

0.618199650811

$-1.702425824066$

$-0.490020127868$

$-1.150002765442$

1. 517282801295

$-0.881968104425$

$-0.891711766641$

0.976254972494

$-3.107158024252$

$-2.633331987125$

$-0.904641140183$

2.203446588407

0.602045174363

$-0.456869717872$

$-0.877166302558$

$-0.190114930116$

1.064215611894

$-1.599931273394$

$-1.162915402607$

$-2.185410478033$

$-0.101168056238$

$-0.345028459747$

$-1.023761713041$

1.532092153125

$-1.964041884671$

0.032535190738

1.836034053008

$-2.358556557129$

$-2.225482726683$

$-0.590349718956$

$-0.291847497723$

$-1.715545502134$

$-0.668338837052$

$-0.480354869779$

2.413961744599

$-1.544414822767$ 


\begin{tabular}{|c|c|}
\hline & \\
\hline $\mathrm{C}$ & -6.663101900647 \\
\hline $\mathrm{C}$ & -6.162145768937 \\
\hline $\mathrm{C}$ & -4.709559592434 \\
\hline C & -4.333749791729 \\
\hline $\mathrm{C}$ & -7.485181613227 \\
\hline $\mathrm{C}$ & -8.140546423880 \\
\hline 0 & -6.638046155749 \\
\hline $\mathrm{H}$ & -7.268537875162 \\
\hline 0 & -6.987768157465 \\
\hline 0 & -3.854063900801 \\
\hline 0 & -3.932351448821 \\
\hline $\mathrm{H}$ & -6.171012694907 \\
\hline $\mathrm{H}$ & -4.691301913019 \\
\hline $\mathrm{H}$ & -3.524939627463 \\
\hline $\mathrm{H}$ & -8.304071846368 \\
\hline $\mathrm{F}$ & -8.873912335715 \\
\hline $\mathrm{F}$ & -8.969305511887 \\
\hline $\mathrm{F}$ & -7.259197543502 \\
\hline $\mathrm{H}$ & -7.129711200227 \\
\hline $\mathrm{H}$ & -6.778457060947 \\
\hline $\mathrm{H}$ & -3.599676900905 \\
\hline $\mathrm{H}$ & -4.729551629695 \\
\hline & 0097 \\
\hline 0 & -2.470936300537 \\
\hline $\mathrm{C}$ & -3.547462911242 \\
\hline $\mathrm{C}$ & -3.056520699172 \\
\hline $\mathrm{C}$ & -2.262127743018 \\
\hline $\mathrm{C}$ & -1.140580004337 \\
\hline $\mathrm{C}$ & -1.717637192218 \\
\hline $\mathrm{C}$ & -4.353621908646 \\
\hline 0 & -2.195487992348 \\
\hline 0 & -1.757977162103 \\
\hline 0 & -0.496142399933 \\
\hline 0 & -0.659496454498 \\
\hline $\mathrm{H}$ & -4.227354748097 \\
\hline $\mathrm{H}$ & -3.918343974324 \\
\hline $\mathrm{H}$ & -2.943585767567 \\
\hline $\mathrm{H}$ & -0.447220030915 \\
\hline $\mathrm{H}$ & -2.379100682074 \\
\hline $\mathrm{F}$ & -4.865428985700 \\
\hline $\mathrm{F}$ & -5.381276731916 \\
\hline $\mathrm{F}$ & -3.629480875441 \\
\hline $\mathrm{H}$ & -2.709499765391 \\
\hline $\mathrm{H}$ & -1.168532780629 \\
\hline $\mathrm{H}$ & 0.306913403551 \\
\hline $\mathrm{H}$ & -0.969398293443 \\
\hline $5 b$ & 0138 \\
\hline 0 & -6.329936706305 \\
\hline $\mathrm{C}$ & -5.746195233228 \\
\hline $\mathrm{C}$ & -5.373232703093 \\
\hline $\mathrm{C}$ & -5.989202594969 \\
\hline C & -5.982186723003 \\
\hline $\mathrm{C}$ & -6.771911965458 \\
\hline $\mathrm{C}$ & -7.150748679483 \\
\hline 0 & -7.946036825853 \\
\hline $\mathrm{H}$ & -4.835852342110 \\
\hline 0 & $71350^{\circ}$ \\
\hline
\end{tabular}

3.409492811947

2. 241302601382

2.081991523472

3. 522740378824

4.398651354335

5.477801083980

5.009610835519

3.021984578279

1.120598847993

1.502912106049

4.237715037133

2. 514627109575

1.454465661652

3.599636154089

3.846061440359

6.305676336784

4.931369251691

6.236361006693

5.264169033632

0.441046208365

2.205038572299

4.593712031828

5.525538620876

5.024890623916

4.160613250843

3.010790112822

3.563118175896

4.483990986108

6.234708418418

4.875242613199

2.189728628899

2.449402499324

5.055913040887

4. 424163918681

3. 753775128691

2.433819307895

4.146062551172

3.918657370145

6.891488656139

5.851689219939

7.118742616339

5.415562339899

1.543471537207

2.750585426423

5.430644956279

3.583077815221

3.817049250249

2. 433848808979

1. 461950998741

2. 285936276311

4.551946550867

5.910592364237

3. 816298417965

4.418507718485

2. 355745858973
$-1.007559657263$

$-0.147332268432$

$-0.606893819952$

$-0.970910810974$

$-0.185778081385$

$-1.039920222025$

0.759295265673

$-1.833708079413$

$-0.373174179890$

0.344388969224

0.174142216581

0.917089148681

$-1.504230093241$

$-1.699037073558$

0.291911848995

$-0.272293425008$

$-1.947193266459$

$-1.702939882328$

1.550862594159

0.280113617905

0.963392592735

0.607189428740

0.494574497051

$-0.262967296773$

$-1.440586245132$

$-0.828494134439$

0.041254038553

1. 110852006593

$-0.701944400397$

$-2.298986761361$

$-1.854980540454$

0.625400876976

1.793299326297

0.361933718671

$-1.983213171722$

$-0.183152266974$

$-0.579384290264$

1. 787259167450

0.349242538911

$-1.480297741741$

$-1.405879699195$

$-2.910992138721$

$-1.441226309515$

1.069882364581

2. 627906461606

0.335699244303

$-0.948905845276$

$-1.529658528351$

$-0.503147857818$

0.777686615814

$-1.806057337444$

$-1.220688795672$

$-2.018480023802$

$-0.853953736328$

$-1.611774120431$ 


\begin{tabular}{|c|c|}
\hline & \\
\hline 0 & -7.361966323591 \\
\hline 0 & -4.702086145705 \\
\hline $\mathrm{H}$ & -5.832407377141 \\
\hline $\mathrm{H}$ & -5.431883971828 \\
\hline $\mathrm{H}$ & -6.752625416129 \\
\hline $\mathrm{H}$ & -6.311542494936 \\
\hline $\mathrm{F}$ & -7.888321647635 \\
\hline $\mathrm{F}$ & -6.056313503204 \\
\hline $\mathrm{F}$ & -7.871249518843 \\
\hline $\mathrm{H}$ & -8.030868081208 \\
\hline $\mathrm{H}$ & -3.724358618344 \\
\hline $\mathrm{H}$ & -7.442514659898 \\
\hline $\mathrm{H}$ & -4.704123397823 \\
\hline $5 a$ & 0601 \\
\hline 0 & -6.908571243072 \\
\hline $\mathrm{C}$ & -5.839345435254 \\
\hline $\mathrm{C}$ & -5.166495838298 \\
\hline $\mathrm{C}$ & -5.710175508065 \\
\hline $\mathrm{C}$ & -7.095799656678 \\
\hline $\mathrm{C}$ & -6.387526596518 \\
\hline $\mathrm{C}$ & -6.994615156225 \\
\hline 0 & -7.333361954626 \\
\hline $\mathrm{H}$ & -5.131367973177 \\
\hline 0 & -3.764140783524 \\
\hline 0 & -5.848132921119 \\
\hline 0 & -7.993242635536 \\
\hline $\mathrm{H}$ & -5.515001191006 \\
\hline $\mathrm{H}$ & -5.108324029125 \\
\hline $\mathrm{H}$ & -7.519622512042 \\
\hline $\mathrm{H}$ & -5.544910077326 \\
\hline $\mathrm{F}$ & -7.288462863441 \\
\hline $\mathrm{F}$ & -6.140457058913 \\
\hline $\mathrm{F}$ & -8.131099002282 \\
\hline $\mathrm{H}$ & -7.834237647759 \\
\hline $\mathrm{H}$ & -3.386922848888 \\
\hline $\mathrm{H}$ & -4.989000398078 \\
\hline $\mathrm{H}$ & -7.741186669046 \\
\hline $5 \mathrm{~b}$ & 0090 \\
\hline O & -6.213098340984 \\
\hline C & -5.778426210584 \\
\hline C & -5.345503970971 \\
\hline $\mathrm{C}$ & -5.921489308463 \\
\hline $\mathrm{C}$ & -5.804558276178 \\
\hline C & -6.906736379636 \\
\hline $\mathrm{C}$ & -7.303725069224 \\
\hline O & -8.037640053453 \\
\hline $\mathrm{H}$ & -4.917520385264 \\
\hline 0 & -3.933708616720 \\
\hline 0 & -7.253925349281 \\
\hline O & -4.514281883516 \\
\hline $\mathrm{H}$ & -5.728855539196 \\
\hline $\mathrm{H}$ & -5.327275982504 \\
\hline $\mathrm{H}$ & -6.490260522579 \\
\hline $\mathrm{H}$ & -6.547845595843 \\
\hline $\mathrm{F}$ & -7.851709133212 \\
\hline $\mathrm{F}$ & -8.210221896375 \\
\hline $\mathrm{F}$ & -6.241086299338 \\
\hline
\end{tabular}

1. 249370985003

2. 242235365847

2. 277324795056

0.525577152974

1.979733174753

4.770403732626

6.615267414974

6.646092907175

5.822992669596

3.132657363717

1.567283539917

0.677362030615

2.740944807720

3.785366060981

4.055416282501

2. 698749979701

1.802596402470

2.402442178094

4.713851504582

6.086617925838

3.927631546253

4.733578560855

2.751719126157

0.446770076278

1.969586297014

2.303109010214

1. 926437378217

2.223086897519

4.892816820149

6.722861869059

6.873835803319

6.019327519375

3.398371159948

3.057065381720

0.084802329288

1.071959890471

3.728147993665

3.908342960808

2.507457165403

1. 568004844421

2.449494742808

4.520450771047

5.909697581539

3.682453946237

4.587466879721

2.357695625579

1.198672152961

2.469910347613

2.275802543314

0.656137716939

2.163466876331

4.653246310010

5.903801493164

6.457067486383

6.732678263816
$-0.795798808233$

1. 331764720246

$-2.513506234411$

$-0.412856984369$

1. 490716090588

$-2.777340654058$

$-2.099774559274$

$-0.945937788444$

$-0.094640491890$

$-1.330447003682$

$-2.113030484121$

$-1.570270193284$

2. 159202775938

0.703643383722

$-0.213393123972$

$-0.506429142193$

0.612303120557

0.841269525728

$-1.479284688937$

$-1.199346967917$

$-2.146854361419$

0.275266098244

$-0.603995070345$

0.261449619258

$-0.161448030539$

$-1.465878853720$

1.522953749553

1. 831492307266

$-2.158895545843$

$-2.347695016578$

$-0.518518962415$

$-0.491037711747$

$-1.497629353061$

0.232494462794

0.008096615239

$-0.433562053676$

0.412538848322

$-0.934834025410$

$-1.440629560867$

$-0.371671484870$

0.877740936255

$-1.756444350963$

$-1.264266289401$

$-1.700536428191$

$-0.965608362377$

$-1.376305206105$

$-0.625099799866$

1. 414784088701

$-2.439843748702$

$-0.289022601778$

1.675998540612

$-2.784080805339$

$-0.044808050080$

$-2.097858049991$

$-1.231357988450$ 


\begin{tabular}{|c|c|}
\hline & \\
\hline $\mathrm{H}$ & -8.565704906810 \\
\hline $\mathrm{H}$ & -3.509277150854 \\
\hline $\mathrm{H}$ & -7.755828059608 \\
\hline $\mathrm{H}$ & -3.869921069405 \\
\hline $5 a_{-}$ & 0789 \\
\hline- & -6.064770056396 \\
\hline $\mathrm{C}$ & -6.895795562975 \\
\hline $\mathrm{C}$ & -6.040013372373 \\
\hline $\mathrm{C}$ & -4.631529504540 \\
\hline $\mathrm{C}$ & -4.908989210867 \\
\hline $\mathrm{C}$ & -7.393341694390 \\
\hline $\mathrm{C}$ & -8.210808555579 \\
\hline 0 & -6.379843424500 \\
\hline $\mathrm{H}$ & -7.761114399795 \\
\hline 0 & -6.353145139122 \\
\hline 0 & -4.051336347814 \\
\hline 0 & -3.888897971914 \\
\hline $\mathrm{H}$ & -6.105090002255 \\
\hline $\mathrm{H}$ & -3.987307573740 \\
\hline $\mathrm{H}$ & -5.136101097244 \\
\hline $\mathrm{H}$ & -8.090369174103 \\
\hline $\mathrm{F}$ & -9.192984864871 \\
\hline $\mathrm{F}$ & -7.478351223410 \\
\hline $\mathrm{F}$ & -8.799788696497 \\
\hline $\mathrm{H}$ & -5.517322302737 \\
\hline $\mathrm{H}$ & -7.226818734751 \\
\hline $\mathrm{H}$ & -3.635026079835 \\
\hline $\mathrm{H}$ & -3.535255010292 \\
\hline & 0056 \\
\hline 0 & -2.452751853798 \\
\hline $\mathrm{C}$ & -3.566631790627 \\
\hline $\mathrm{C}$ & -3.091411749580 \\
\hline $\mathrm{C}$ & -2.299800054324 \\
\hline $\mathrm{C}$ & -1.181320830601 \\
\hline $\mathrm{C}$ & -1.742127332175 \\
\hline $\mathrm{C}$ & -4.358603067966 \\
\hline 0 & -2.337233736512 \\
\hline 0 & -1.787633192040 \\
\hline 0 & -0.587354701012 \\
\hline 0 & -0.677754319720 \\
\hline $\mathrm{H}$ & -4.234640314840 \\
\hline $\mathrm{H}$ & -3.949385300551 \\
\hline $\mathrm{H}$ & -2.989378698871 \\
\hline $\mathrm{H}$ & -0.445189217854 \\
\hline $\mathrm{H}$ & -2.434889913142 \\
\hline $\mathrm{F}$ & -3.603389523706 \\
\hline $\mathrm{F}$ & -4.929730149044 \\
\hline $\mathrm{F}$ & -5.342013901538 \\
\hline $\mathrm{H}$ & -1.716801758481 \\
\hline $\mathrm{H}$ & -1.237092565370 \\
\hline $\mathrm{H}$ & 0.220099143499 \\
\hline $\mathrm{H}$ & -0.985865171745 \\
\hline $6 \mathrm{~b}$ & 0143 \\
\hline 0 & -2.489434927493 \\
\hline $\mathrm{C}$ & -3.539027386102 \\
\hline $\mathrm{C}$ & -3.030203727378 \\
\hline $\mathrm{C}$ & -2.18923688284 \\
\hline
\end{tabular}

3.767789725413

2.917969165461

1.988888072707

2. 510457334658

2.888810593046

3.764979820249

4.269250884065

3.827714206943

2.542630613796

4.893332533540

4.369610986316

5.718864247304

3.216001660444

3.581670373981

4.713738281671

2.155198716071

5.355684874829

3. 673505409312

1.701947818699

5.505529836118

3.545482723657

3.702124083848

5.387717591991

5.277323143949

3.850591539709

5.459666792880

2. 955623267585

5.545696679595

5.041175018877

4.150685503698

2. 998638917773

3.531373415974

4.507167027915

6.258625476104

4.884343752817

2.183200142727

2.412674440716

5.074206867975

4.470090761098

3.740461560646

2. 420871984230

4.071022996124

3.994864697997

7.173338424916

6.873980337465

5.897832783121

5.464513745655

1. 504726584396

2.694637269759

5. 492071610419

5.570383630025

5.022727071137

4.071723493640

2.995655667323
$-2.504930783617$

$-2.039005759745$

$-0.889569438733$

0.689110320599

$-0.562664218353$

0.192065190314

1. 387709838756

0.994061457787

0.208826260232

$-0.707730869145$

$-1.886256762103$

$-1.212315275175$

0.583130295008

2. 581675341677

0.048612922462

$-0.631890609293$

1. 517389139171

1. 862146474864

0.871776803826

$-0.120797665406$

$-1.472480374151$

$-2.787390461872$

$-2.541902321377$

$-1.120106214501$

2.892639190664

0.498909572669

$-1.051407716054$

0.375051082730

$-0.333321546856$

$-1.487671916916$

$-0.861331831348$

0.025747734525

1. 054154174115

$-0.785865728742$

$-2.427134600551$

$-1.888437002886$

0.648374030395

1.726701701089

0.328437641403

$-2.027619231407$

$-0.224683371369$

$-0.588378237201$

1.739890013704

$-1.408265112265$

0.263873865902

$-1.624943440575$

$-1.962699147024$

$-1.473177658105$

1.097866203719

2. 541432377665

0.412086736858

$-0.348433341586$

$-1.441855414908$

$-0.756843467774$ 


\begin{tabular}{|c|c|}
\hline & \\
\hline $\mathrm{C}$ & -1.113481719436 \\
\hline $\mathrm{C}$ & -1.753747180078 \\
\hline $\mathrm{C}$ & -4.322683852583 \\
\hline 0 & -2.167746541503 \\
\hline 0 & -1.591520515621 \\
\hline 0 & -0.411271196935 \\
\hline 0 & -0.839317634918 \\
\hline $\mathrm{H}$ & -4.241144165330 \\
\hline $\mathrm{H}$ & -3.882486132480 \\
\hline $\mathrm{H}$ & -2.835186936107 \\
\hline $\mathrm{H}$ & -0.430492987608 \\
\hline $\mathrm{H}$ & -2.432317518297 \\
\hline $\mathrm{F}$ & -3.563003376422 \\
\hline $\mathrm{F}$ & -4.889077172279 \\
\hline $\mathrm{F}$ & -5.308374256213 \\
\hline $\mathrm{H}$ & -2.673431871312 \\
\hline $\mathrm{H}$ & -1.236458379803 \\
\hline $\mathrm{H}$ & -0.153680013324 \\
\hline $\mathrm{H}$ & -0.137775625931 \\
\hline $5 a_{-}$ & 0112 \\
\hline 0 & -5.017281096075 \\
\hline $\mathrm{C}$ & -6.173482740478 \\
\hline $\mathrm{C}$ & -5.734215120258 \\
\hline $\mathrm{C}$ & -4.206446522946 \\
\hline $\mathrm{C}$ & -4.019784382050 \\
\hline $\mathrm{C}$ & -7.272065901653 \\
\hline $\mathrm{C}$ & -8.632746819025 \\
\hline 0 & -7.385965182836 \\
\hline $\mathrm{H}$ & -6.475983073218 \\
\hline 0 & -6.115053905358 \\
\hline O & -3.562809962006 \\
\hline 0 & -4.244716950326 \\
\hline $\mathrm{H}$ & -6.091069824695 \\
\hline $\mathrm{H}$ & -3.821728187741 \\
\hline $\mathrm{H}$ & -3.058691765017 \\
\hline $\mathrm{H}$ & -7.066085088850 \\
\hline $\mathrm{F}$ & -8.631750998530 \\
\hline $\mathrm{F}$ & -8.987595385132 \\
\hline $\mathrm{F}$ & -9.597526987820 \\
\hline $\mathrm{H}$ & -6.485206956596 \\
\hline $\mathrm{H}$ & -7.030368610034 \\
\hline $\mathrm{H}$ & -3.702422144015 \\
\hline $\mathrm{H}$ & -3.948502395342 \\
\hline $5 \mathrm{~b}$ & 0070 \\
\hline 0 & -6.285469852324 \\
\hline C & -5.799302920202 \\
\hline $\mathrm{C}$ & -5.454202032484 \\
\hline $\mathrm{C}$ & -5.970619152936 \\
\hline $\mathrm{C}$ & -5.883969012772 \\
\hline C & -6.889816745035 \\
\hline $\mathrm{C}$ & -7.258778564532 \\
\hline O & -8.061854017979 \\
\hline $\mathrm{H}$ & -4.891192570857 \\
\hline O & -4.059220417009 \\
\hline 0 & -7.333359029090 \\
\hline O & -4.564520270942 \\
\hline $\mathrm{H}$ & -5.993635086982 \\
\hline
\end{tabular}

3.631594362337

4.582588017240

6.203843235759

4.703811259925

2.130623661867

2.636814461224

5.234024872257

4.466575908794

3.603495818551

2. 373669838466

4.220267643319

4.036895060821

7.049240532875

6.914534205999

5.774402173232

5.109669984951

2.684956353188

1.956336602482

5. 611166144589

4.858941771093

4.024726831749

2. 845737692032

2. 967712440909

4. 477108651813

4.915506310520

4.248051991793

6.120568427586

3. 662753676514

1. 583613713409

2.411837044595

5.017485977586

2.981499492268

2.465860800851

4.792436398232

5.092314863507

3.071913951075

3. 982224133881

5.006805728106

6.446754423538

1.403364475523

3.011966791011

5.936114412411

3.725252154970

3. 901772451021

2. 495051922048

1. 567695184362

2. 457773465331

4.586474022924

5.958316363027

3.826438972299

4.514029066867

2.427933261002

1.317956114171

2. 455905653168

2. 277257741538
0.126821595780

1. 133760013180

$-0.895595150404$

$-2.368750747449$

$-1.698290006358$

0.836853079878

1.936871470131

0.293325505104

$-1.947248723500$

$-0.126179955943$

$-0.507165512850$

1.804460912721

$-1.607152456473$

0.090511886165

$-1.703733481496$

$-3.083400239079$

$-2.410824368496$

0.198331662848

1. 386950003650

$-1.391991266551$

$-1.212528435290$

$-0.320139909649$

$-0.328240318478$

$-0.469382356939$

$-0.617600358436$

$-0.707582540191$

$-1.327091224760$

$-2.200884628875$

$-0.817308184858$

0.785294157751

0.805431894493

0.709565716594

$-1.221315951003$

$-0.879279875598$

0.448058273924

$-0.042616775566$

$-1.973295231529$

$-0.172204023006$

$-1.473280462779$

$-0.569382404923$

1. 533127686020

0.840346219652

0.432191248172

$-0.900947524991$

$-1.441413168108$

$-0.329136458687$

0.909462927579

$-1.718430628698$

$-1.157948061353$

$-1.834934949042$

$-0.900785396994$

$-1.634490366919$

$-0.636051438231$

1. 365250957672

$-2.370358859889$ 


\begin{tabular}{|c|c|c|c|}
\hline $\mathrm{H}$ & -5.388097828020 & 0.646566926202 & -0.240227339114 \\
\hline $\mathrm{H}$ & -6.589699482073 & 2.185831460404 & 1.701020661827 \\
\hline $\mathrm{H}$ & -6.491330757020 & 4.778346838539 & -2.722393752449 \\
\hline $\mathrm{F}$ & -6.163015200027 & 6.717453797041 & -0.964145696431 \\
\hline $\mathrm{F}$ & -7.918936399779 & 5.898299612360 & 0.006005098182 \\
\hline $\mathrm{F}$ & -8.051710548878 & 6.623706393988 & -2.019688445030 \\
\hline $\mathrm{H}$ & -8.083021395491 & 3.144196137854 & -1.140732874055 \\
\hline $\mathrm{H}$ & -3.847888089600 & 1.620989476635 & -2.120308843266 \\
\hline $\mathrm{H}$ & -7.724835282654 & 0.749367331534 & 0.039764660369 \\
\hline $\mathrm{H}$ & -4.506825343314 & 3.003285652716 & 2.159398249457 \\
\hline \multicolumn{4}{|c|}{ 6b confs 0687} \\
\hline 0 & -2.060822804203 & 4.422839510339 & -1.326304918038 \\
\hline $\mathrm{C}$ & -2.256693605087 & 5.525376638188 & -0.472384281879 \\
\hline $\mathrm{C}$ & -3.464219884393 & 5.351080673947 & 0.447506771763 \\
\hline $\mathrm{C}$ & -3.290316634508 & 4.028857572090 & 1.202341101257 \\
\hline $\mathrm{C}$ & -3.000250610382 & 2.878479211829 & 0.243973791132 \\
\hline $\mathrm{C}$ & -1.789157785127 & 3.213398397900 & -0.622468646646 \\
\hline $\mathrm{C}$ & -2.360787677163 & 6.740680021150 & -1.382115436983 \\
\hline 0 & -4.645248491044 & 5.331409781756 & -0.321543209324 \\
\hline 0 & -4.489799168633 & 3.687009944067 & 1.878463329702 \\
\hline 0 & -2.721216512581 & 1.695499274152 & 0.957111659108 \\
\hline 0 & -1.492749356811 & 2.236205108154 & -1.551468753871 \\
\hline $\mathrm{H}$ & -1.368516226443 & 5.694143888202 & 0.156509675149 \\
\hline $\mathrm{H}$ & -3.479929865879 & 6.181662250734 & 1.167127748805 \\
\hline $\mathrm{H}$ & -2.455812514124 & 4.114692212140 & 1.910894382569 \\
\hline $\mathrm{H}$ & -3.867546957856 & 2.744079376819 & -0.421561965413 \\
\hline $\mathrm{H}$ & -0.891586634681 & 3.340737022232 & -0.000932455889 \\
\hline $\mathrm{F}$ & -3.258812296123 & 6.593477928814 & -2.361238386545 \\
\hline $\mathrm{F}$ & -1.178532452083 & 6.996577816500 & -1.971390585982 \\
\hline $\mathrm{F}$ & -2.698266009303 & 7.832395290182 & -0.674587475143 \\
\hline $\mathrm{H}$ & -5.344312060665 & 4.969254131769 & 0.243340473151 \\
\hline $\mathrm{H}$ & -4.511564195087 & 4.107922140056 & 2.747222374099 \\
\hline $\mathrm{H}$ & -3.446042744806 & 1.557324369665 & 1.583011141109 \\
\hline $\mathrm{H}$ & -2.295815513018 & 1.997897439316 & -2.036506332131 \\
\hline \multicolumn{4}{|c|}{$6 a$ confs 0060} \\
\hline $0^{-}$ & -2.293418607400 & 5.835374484660 & 0.169524413634 \\
\hline $\mathrm{C}$ & -3.389983738367 & 4.963873502553 & 0.350604330736 \\
\hline $\mathrm{C}$ & -3.536009284064 & 3.985964383080 & -0.828643099955 \\
\hline $\mathrm{C}$ & -2.224726102695 & 3.193012149494 & -0.885539023775 \\
\hline C & -1.053670276015 & 4.159652921379 & -1.032934646059 \\
\hline $\mathrm{C}$ & -1.046250756418 & 5.153626482091 & 0.127096000451 \\
\hline $\mathrm{C}$ & -4.602948076608 & 5.850297262885 & 0.559440406180 \\
\hline 0 & -3.688940347849 & 4.647385858725 & -2.065482567376 \\
\hline 0 & -2.158938589247 & 2.315704550387 & -1.991579225900 \\
\hline 0 & 0.176997745161 & 3.473794699270 & -1.051625495312 \\
\hline 0 & -0.811287339624 & 4.454408694561 & 1.310562152993 \\
\hline $\mathrm{H}$ & -3.278605919126 & 4.386448777526 & 1.278633586236 \\
\hline $\mathrm{H}$ & -4.372366172831 & 3.301636256160 & -0.634467379049 \\
\hline $\mathrm{H}$ & -2.107890781210 & 2.637738624035 & 0.054216467097 \\
\hline $\mathrm{H}$ & -1.193303687310 & 4.735708853287 & -1.956892923549 \\
\hline $\mathrm{H}$ & -0.302733658734 & 5.938542871587 & -0.034110713433 \\
\hline $\mathrm{F}$ & -4.455142012508 & 6.644958177356 & 1.629277927413 \\
\hline $\mathrm{F}$ & -5.699720488292 & 5.098204269631 & 0.761962115469 \\
\hline $\mathrm{F}$ & -4.860250224887 & 6.648748133628 & -0.490941301637 \\
\hline $\mathrm{H}$ & -4.585092051453 & 4.994658987419 & -2.149439068650 \\
\hline $\mathrm{H}$ & -2.629405476179 & 1.498556353063 & -1.787011542675 \\
\hline $\mathrm{H}$ & 0.098003797112 & 2.772545101706 & -1.713618930605 \\
\hline
\end{tabular}




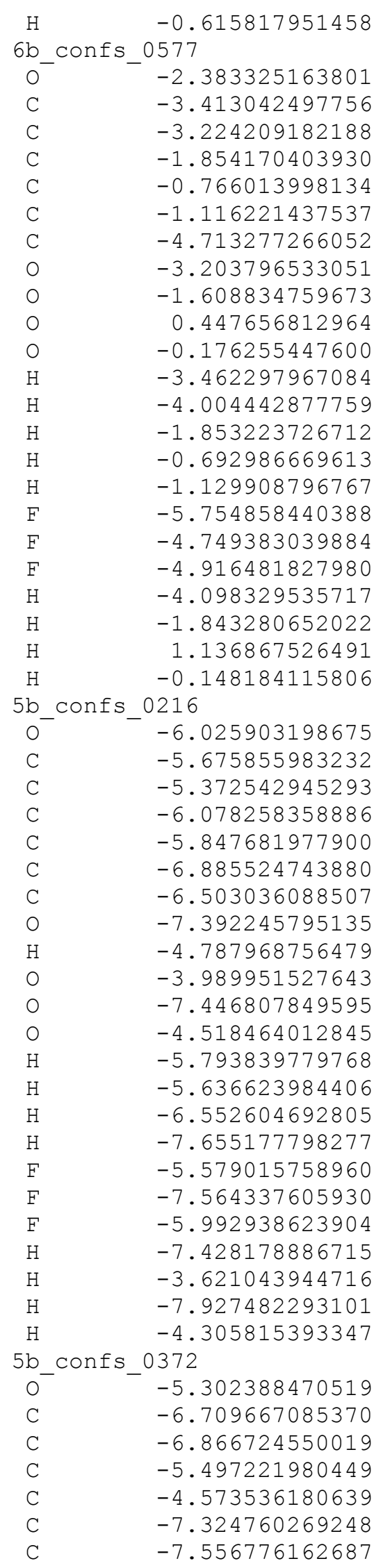

$\begin{array}{lr}5.082158605519 & 2.018468517764 \\ 5.633253431016 & -1.355219302580 \\ 5.433184114381 & -0.416416726990 \\ 4.133786353804 & 0.376538809389 \\ 4.172646729450 & 1.063555218876 \\ 4.497114166347 & 0.040858302778\end{array}$

$5.773695619695 \quad-0.729077119632$

$5.477379573051 \quad-1.201495623851$

$2.987581886941 \quad-0.454703148888$

$2.955867808253 \quad 1.728148685455$

$4.677197271187 \quad 0.736096122729$

$6.061893078751 \quad-1.706100141074$

$6.272169588682 \quad 0.295993608280$

$4.054661681391 \quad 1.141846883204$

$4.957023773349 \quad 1.829365116541$

$3.668861214640 \quad-0.682038873258$

$6.634578357988 \quad-0.045440661614$

$5.214835913018 \quad-0.392468113036$

$4.585302755296 \quad-2.201923039102$

$6.687499740336 \quad-1.742679798778$

$2.658585884088 \quad-0.605066195219$

$2.244370576976 \quad 1.113033833209$

$\begin{array}{ll}4.899079011392 & 0.095531651943\end{array}$

$5.332431469970 \quad-2.343339488381$

$\begin{array}{ll}3.922363607370 & 0.264842071511\end{array}$

$3.878837042537 \quad-1.129485004956$

$2.399925555686 \quad-1.452531939261$

$1.666582041695 \quad-0.303226365251$

$2.647572934343 \quad 0.845322736236$

$4.461712164738 \quad-1.878287502782$

$4.934349571730 \quad-3.270231061423$

$5.595657691478 \quad-1.222504048874$

$4.499535130528 \quad-1.293221392543$

$2.129024496529 \quad-1.502547741309$

$1.593884898054 \quad-0.640085513312$

$2.465574892881 \quad 1.266517110160$

$2.093708694282 \quad-2.411979806649$

$0.684715914328 \quad-0.109701691420$

$2.567269335743 \quad 1.677323081201$

$3.686273420388 \quad-1.993312959246$

$5.908249219328 \quad-3.239016281596$

$5.416177216119 \quad-3.934707391907$

$3.927771136793 \quad-4.004228839482$

$5.380571353756 \quad-0.278311088744$

$2.272535151664 \quad-0.616785582308$

$\begin{array}{ll}1.119866939908 & 0.050454827271\end{array}$

$3.118241590121 \quad 1.947304384688$

$3.738609767286 \quad-1.162572081828$

$3.506910606768 \quad-1.051858199801$

$2.187396848915 \quad-0.271066268887$

$2.019298787629 \quad 0.403356264081$

$2.615533242019 \quad-0.669573108523$

$4.730312368536 \quad-0.352319866494$

$5.884280129608 \quad-1.322246655621$ 


\begin{tabular}{|c|c|c|c|}
\hline & & & \\
\hline O & -6.513072369183 & 5.182054499583 & 0.704319167940 \\
\hline $\mathrm{H}$ & -7.155196715246 & 3.379045009809 & -2.044145009426 \\
\hline 0 & -7.063917876242 & 1.174595133834 & -1.250518101753 \\
\hline 0 & -5.405598863671 & 2.697103220593 & 1.633403709013 \\
\hline O & -4.267437224389 & 1.713563950011 & -1.688351799122 \\
\hline $\mathrm{H}$ & -7.686694999805 & 2.209820275853 & 0.454184133200 \\
\hline $\mathrm{H}$ & -5.262968518088 & 0.968678388471 & 0.588924034580 \\
\hline $\mathrm{H}$ & -3.632032702956 & 2.992677373404 & -0.268377625393 \\
\hline $\mathrm{H}$ & -8.304001039973 & 4.474628715477 & 0.061751994921 \\
\hline $\mathrm{F}$ & -8.161806424338 & 6.916073861301 & -0.715407764255 \\
\hline F & -8.330888389821 & 5.510359194700 & -2.353353399974 \\
\hline $\mathrm{F}$ & -6.400383397173 & 6.347236143996 & -1.829619844525 \\
\hline $\mathrm{H}$ & -5.633060047253 & 5.359534834770 & 0.329615756050 \\
\hline $\mathrm{H}$ & -7.246502922882 & 0.333946354803 & -0.809683959343 \\
\hline $\mathrm{H}$ & -5.797362027926 & 3.583172733131 & 1.555308475370 \\
\hline $\mathrm{H}$ & -5.083001782122 & 1.259168559504 & -1.956769850211 \\
\hline $6 a_{-}$ & 0014 & & \\
\hline 0 & -2.314871848622 & 5.830506060883 & 0.178060406054 \\
\hline C & -3.404457068842 & 4.951142418510 & 0.354866674260 \\
\hline C & -3.535937178268 & 3.992113440327 & -0.838316585800 \\
\hline $\mathrm{C}$ & -2.241352076183 & 3.166606930088 & -0.883407727757 \\
\hline $\mathrm{C}$ & -1.043372902577 & 4.110706801656 & -1.006897656151 \\
\hline $\mathrm{C}$ & -1.071600145123 & 5.159786675235 & 0.113228618477 \\
\hline $\mathrm{C}$ & -4.624064506684 & 5.826429782249 & 0.572610041281 \\
\hline 0 & -3.640830205645 & 4.675122472006 & -2.068318269680 \\
\hline 0 & -2.211785421857 & 2.288558464542 & -1.985666803323 \\
\hline 0 & 0.188535804475 & 3.426229680222 & -0.979984507993 \\
\hline 0 & -0.799069553494 & 4.470920661778 & 1.303487017554 \\
\hline $\mathrm{H}$ & -3.288243563483 & 4.365515912191 & 1.277242820947 \\
\hline $\mathrm{H}$ & -4.390975693147 & 3.321731307145 & -0.677717198724 \\
\hline $\mathrm{H}$ & -2.157834253098 & 2.607170997473 & 0.059613905482 \\
\hline $\mathrm{H}$ & -1.104118233798 & 4.623427714654 & -1.970658070539 \\
\hline $\mathrm{H}$ & -0.333337926418 & 5.943385320634 & -0.073013767092 \\
\hline $\mathrm{F}$ & -4.471569305636 & 6.631954195251 & 1.632910089239 \\
\hline $\mathrm{F}$ & -5.709852347697 & 5.065212324000 & 0.793684828791 \\
\hline $\mathrm{F}$ & -4.900897268458 & 6.613003670412 & -0.483001198227 \\
\hline $\mathrm{H}$ & -4.515594635230 & 5.071926555508 & -2.157819308371 \\
\hline $\mathrm{H}$ & -2.811060033974 & 1.550352816695 & -1.820393450642 \\
\hline $\mathrm{H}$ & 0.343099377132 & 3.137850357478 & -0.068350120728 \\
\hline $\mathrm{H}$ & -0.532511013372 & 5.093745441063 & 1.991940262946 \\
\hline $5 a_{-}$ & 0211 & & \\
\hline 0 & -5.007235398196 & 4.993874769037 & -1.388107653199 \\
\hline C & -6.167937313242 & 4.158841033941 & -1.273652243518 \\
\hline C & -5.776173454954 & 3.004684407093 & -0.329342565980 \\
\hline $\mathrm{C}$ & -4.248362118091 & 3.085521524247 & -0.333809770672 \\
\hline $\mathrm{C}$ & -4.019889324557 & 4.588493915714 & -0.459875258212 \\
\hline $\mathrm{C}$ & -7.348250861957 & 4.966507665838 & -0.735964659652 \\
\hline $\mathrm{C}$ & -7.764533604918 & 6.089091285415 & -1.682965381175 \\
\hline 0 & -7.112285286070 & 5.509538000876 & 0.533008741181 \\
\hline $\mathrm{H}$ & -6.412578711059 & 3.769476976463 & -2.268170141133 \\
\hline 0 & -6.309329616184 & 1.751819307954 & -0.686559393724 \\
\hline 0 & -3.622763663633 & 2.553957071850 & 0.802472705822 \\
\hline 0 & -4.234292351762 & 5.198277132459 & 0.788009484703 \\
\hline $\mathrm{H}$ & -6.131721369752 & 3.200613208763 & 0.687076227140 \\
\hline $\mathrm{H}$ & -3.867914372990 & 2.619073170325 & -1.254821479776 \\
\hline $\mathrm{H}$ & -3.047944173871 & 4.865718136084 & -0.870275630061 \\
\hline $\mathrm{H}$ & -8.213431009546 & 4.295286114112 & -0.668300819541 \\
\hline
\end{tabular}




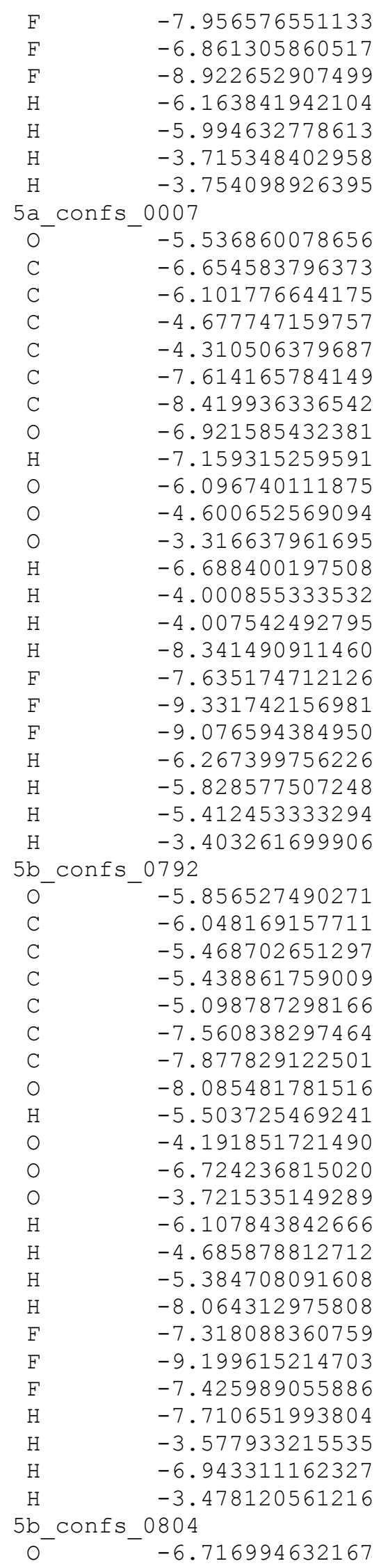

5.631909107755

7.077880205745

6.646414658022

5.713378929029

1.500104968634

1.592818373371

6.035620037273

3.847299723750

3.197000004172

2.306590796256

2. 830138538890

3.287360923495

4.285323317955

4. 912094633781

5.303135982889

2. 550408728992

0.982068091236

3.925385465045

4.240077263223

2.387276263754

2.056438367096

2.453866223223

3.851356407991

5.595039220293

5.775709448589

3.980949623292

5.667557148955

0.378009734573

4.458109006473

4.778805086077

5.251246881849

4.400902367972

3.021764572915

3.103562944323

4.573359537989

4.413499359660

3.966926813308

5.711520320351

4.822084255780

2.796804672085

2.907051419162

4.706932531714

2.201614186131

2.439253780709

4.978215024930

3.717285457561

4.767607925123

3. 962249280359

2.720447413555

6.098750377191

3. 441386264706

1.967159908755

5.641374703871

4.447456056259
$-2.935105063052$

$-1.749867246104$

$-1.284196968956$

0.626584834201

$-1.565780079212$

0.785678780500

0.827063580421

$-1.283370464329$

$-0.688382271200$

0.448376099701

0.639614132156

$-0.779942067876$

$-0.190820846213$

$-1.323822946296$

0.492406364185

$-1.414768598805$

$-0.047433298323$

1.531011029913

$-0.815933130313$

1.371200180546

1.007695846517

$-1.421032365772$

0.500812323666

$-2.175614486504$

$-0.851252499776$

$-2.033609849562$

$-0.128538325604$

0.657178570587

1. 473114300382

$-0.011887697078$

0.387201196018

$-0.756845163566$

$-0.372332047869$

1.165891138538

1.367643347946

$-1.028499458030$

$-2.445768815988$

$-0.918596205863$

$-1.609321994688$

$-0.927969640529$

1. 715282810454

1.120203021460

$-0.707513846714$

1.599910909830

2. 341056465746

$-0.343479361019$

$-3.366962846689$

$-2.674659840651$

$-2.677864663356$

$-0.113347678008$

$-0.542138861440$

1.686980023122

1.167131511295

0.983106224985 


\begin{tabular}{|c|c|}
\hline & \\
\hline $\mathrm{C}$ & -6.157836367250 \\
\hline $\mathrm{C}$ & -5.722823984671 \\
\hline $\mathrm{C}$ & -6.540598470448 \\
\hline C & -6.538137565871 \\
\hline $\mathrm{C}$ & -7.271558640349 \\
\hline $\mathrm{C}$ & -6.715886276435 \\
\hline 0 & -7.919214801096 \\
\hline $\mathrm{H}$ & -5.292022980335 \\
\hline 0 & -4.332660579061 \\
\hline 0 & -7.885324932986 \\
\hline 0 & -5.273127924529 \\
\hline $\mathrm{H}$ & -5.977738265987 \\
\hline $\mathrm{H}$ & -6.071322265111 \\
\hline $\mathrm{H}$ & -7.350584101687 \\
\hline $\mathrm{H}$ & -7.980516439352 \\
\hline $\mathrm{F}$ & -6.068678367474 \\
\hline $\mathrm{F}$ & -5.844370003129 \\
\hline $\mathrm{F}$ & -7.691837803585 \\
\hline $\mathrm{H}$ & -8.088404815700 \\
\hline $\mathrm{H}$ & -4.105714949446 \\
\hline $\mathrm{H}$ & -7.942802121376 \\
\hline $\mathrm{H}$ & -5.204843711956 \\
\hline $5 b$ & 0054 \\
\hline 0 & -5.995052640839 \\
\hline $\mathrm{C}$ & -5.708719625539 \\
\hline $\mathrm{C}$ & -5.361861041986 \\
\hline $\mathrm{C}$ & -6.058829025177 \\
\hline $\mathrm{C}$ & -5.837377840463 \\
\hline $\mathrm{C}$ & -6.963586508671 \\
\hline $\mathrm{C}$ & -6.631777431471 \\
\hline 0 & -7.503874099899 \\
\hline $\mathrm{H}$ & -4.849964534359 \\
\hline 0 & -3.951283051249 \\
\hline 0 & -7.424659241289 \\
\hline 0 & -4.583971141830 \\
\hline $\mathrm{H}$ & -5.718970209764 \\
\hline $\mathrm{H}$ & -5.609098906563 \\
\hline $\mathrm{H}$ & -6.582749519269 \\
\hline $\mathrm{H}$ & -7.700005814062 \\
\hline $\mathrm{F}$ & -7.726355716735 \\
\hline $\mathrm{F}$ & -6.095768842793 \\
\hline $\mathrm{F}$ & -5.749433241321 \\
\hline $\mathrm{H}$ & -7.463863621049 \\
\hline $\mathrm{H}$ & -3.677785647949 \\
\hline $\mathrm{H}$ & -7.897798367594 \\
\hline $\mathrm{H}$ & -3.914313930130 \\
\hline $5 b$ & 0525 \\
\hline 0 & -4.821647458047 \\
\hline $\mathrm{C}$ & -6.125802156980 \\
\hline $\mathrm{C}$ & -5.976381428943 \\
\hline $\mathrm{C}$ & -4.727768145402 \\
\hline C & -3.875151086822 \\
\hline $\mathrm{C}$ & -7.062420693013 \\
\hline $\mathrm{C}$ & -8.511998629563 \\
\hline 0 & -6.774408640883 \\
\hline $\mathrm{H}$ & -6.441804570676 \\
\hline 0 & . \\
\hline
\end{tabular}

4.278799012950

2. 800032863274

2.148891515977

3.265954946468

4.691127801393

5.022194883305

5.859378467021

4.941728528144

2. 632015231221

1.956395288784

3.246573961099

2. 362059225115

1.234564027776

3.215085966425

3.860586700161

3. 968735513092

6.043003322012

5.364959164066

5.751487572270

2.898884056098

1.195338622561

3.989747274526

3.865512310763

3. 850312239370

2.396859811683

1.622277456266

2. 567236198190

4.393299115339

4.921318941624

5.481863926570

4.502850973674

2.272195716063

1.537783599726

2. 410898347880

2.092721932945

0.638356191171

2.460201498878

3.588471995696

5.374742871634

3.956360577058

5.932601508977

5.252514965811

1. 404743734181

1.025486228107

2. 387289858395

4.246628844454

3.709053621633

2.802941370773

3.376153892924

3.742957363295

4.913716007983

4.534117374594

5.934384431713

3.123011984493

1. 492188947418
$-0.331259321520$

$-0.429512785025$

0.702289225160

1.736125680524

$-1.306827325112$

$-2.681665261087$

$-0.873321736387$

$-0.438126457465$

$-0.259473647136$

0.318495241962

2. 348717361444

$-1.397341006754$

1.077051132205

2. 464504264784

$-1.429430259446$

$-3.213718983361$

$-2.644097004189$

$-3.535811887296$

0.074625394806

0.645249240998

$-0.272840967835$

2.963262875745

0.288436713498

$-1.116037692900$

$-1.460233005103$

$-0.337959304398$

0.850993209969

$-1.823500602734$

$-3.208233736455$

$-1.120531372941$

$-1.307928187606$

$-1.346309263028$

$-0.683653840921$

1. 442102661964

$-2.448080545332$

$-0.168829744893$

1.641319700291

$-1.946263582129$

$-3.837896601645$

$-3.979566207092$

$-3.169242226586$

$-0.179280786928$

$-1.672944618701$

$-0.015800735642$

0.738639769310

$-1.471914749915$

$-1.217256859784$

0.010762539604

0.687194453957

$-0.535222535946$

$-1.013037887815$

$-1.260029138933$

$-1.932930590412$

$-2.087202720566$

$-0.473532221333$ 


\begin{tabular}{|c|c|c|c|}
\hline O & -5.135880043495 & 4.526081313022 & 1.396526787798 \\
\hline 0 & -3.169212617192 & 2.656153818858 & -1.050111427762 \\
\hline $\mathrm{H}$ & -6.846847444191 & 2.829431713292 & 0.672167456849 \\
\hline $\mathrm{H}$ & -4.228607482163 & 2.643868732332 & 1.330281778782 \\
\hline $\mathrm{H}$ & -3.150912076135 & 4.536763568616 & -0.342830246393 \\
\hline $\mathrm{H}$ & -6.988195024132 & 5.272341424669 & 0.021869711059 \\
\hline $\mathrm{F}$ & -8.717902498781 & 4.100742042519 & -2.514025500468 \\
\hline $\mathrm{F}$ & -9.334705687896 & 5.575040848202 & -1.060571444972 \\
\hline $\mathrm{F}$ & -8.903310979620 & 3.548431633763 & -0.430134796671 \\
\hline $\mathrm{H}$ & -5.808241873228 & 5.997156069995 & -1.985178644574 \\
\hline $\mathrm{H}$ & -5.739789100722 & 0.867579630489 & 0.263663428998 \\
\hline $\mathrm{H}$ & -4.381446532542 & 4.902262373146 & 1.867345695027 \\
\hline $\mathrm{H}$ & -3.776575527597 & 1.900992991818 & -1.118833086529 \\
\hline \multicolumn{4}{|c|}{ 6a_confs_0049 } \\
\hline 0 & -2.285895429390 & 5.824161469718 & 0.10461430827 \\
\hline $\mathrm{C}$ & -3.377487031021 & 4.950642418419 & 0.338686305795 \\
\hline C & -3.517374342663 & 3.958458860676 & -0.819248100169 \\
\hline $\mathrm{C}$ & -2.196177945920 & 3.172214025317 & -0.886008795982 \\
\hline $\mathrm{C}$ & -1.016774045462 & 4.128774553843 & -1.025857624583 \\
\hline $\mathrm{C}$ & -1.028485522502 & 5.150464977518 & 0.109931730756 \\
\hline $\mathrm{C}$ & -4.588467374549 & 5.849361101216 & 0.523921511763 \\
\hline 0 & -3.827583834161 & 4.610627298074 & -2.032193084911 \\
\hline 0 & -2.131134972393 & 2.306487466920 & -2.001303240054 \\
\hline 0 & 0.210583052231 & 3.440363093744 & -1.012185971863 \\
\hline 0 & -0.813801826852 & 4.485362673236 & 1.312799311520 \\
\hline $\mathrm{H}$ & -3.246680937644 & 4.405256900458 & 1.281453206566 \\
\hline $\mathrm{H}$ & -4.341738273751 & 3.266722604103 & -0.619966698213 \\
\hline $\mathrm{H}$ & -2.077523397087 & 2.607981137001 & 0.049026118957 \\
\hline $\mathrm{H}$ & -1.127056888884 & 4.687408786241 & -1.967077307173 \\
\hline $\mathrm{H}$ & -0.289396862785 & 5.937387413234 & -0.059975640169 \\
\hline $\mathrm{F}$ & -4.724796439838 & 6.757132266605 & -0.452980697092 \\
\hline $\mathrm{F}$ & -4.509754893182 & 6.530735473368 & 1.680280752431 \\
\hline $\mathrm{F}$ & -5.716633466780 & 5.121080978059 & 0.563392227193 \\
\hline $\mathrm{H}$ & -3.283996103435 & 5.406171408581 & -2.121994459258 \\
\hline $\mathrm{H}$ & -2.629169612707 & 1.500254962635 & -1.818566465347 \\
\hline $\mathrm{H}$ & 0.142146702166 & 2.726547920107 & -1.662024603880 \\
\hline $\mathrm{H}$ & -0.611000553393 & 5.128202210929 & 2.005077215437 \\
\hline \multicolumn{4}{|c|}{ 5b confs 0124} \\
\hline 0 & -6.284632511066 & 3.729145313143 & 0.44193433932 \\
\hline $\mathrm{C}$ & -5.804039865695 & 3.918630864367 & -0.888943573430 \\
\hline $\mathrm{C}$ & -5.457777508881 & 2.519277077059 & -1.446321906934 \\
\hline $\mathrm{C}$ & -5.982453872092 & 1.574308483880 & -0.347776700295 \\
\hline $\mathrm{C}$ & -5.893151024169 & 2.449359762212 & 0.901139229066 \\
\hline $\mathrm{C}$ & -6.895410038173 & 4.598231043588 & -1.706005481622 \\
\hline $\mathrm{C}$ & -7.242264443734 & 5.994813592202 & -1.199419981456 \\
\hline 0 & -8.056580430485 & 3.799606264244 & -1.668000602906 \\
\hline $\mathrm{H}$ & -4.898978965094 & 4.536212044183 & -0.889484841481 \\
\hline 0 & -4.059560627769 & 2.460641756709 & -1.634970727447 \\
\hline 0 & -7.321373767780 & 1.200592902263 & -0.591869180478 \\
\hline 0 & -4.574588263467 & 2.442337581362 & 1.366715246660 \\
\hline $\mathrm{H}$ & -5.987481302624 & 2.315942639554 & -2.384931523059 \\
\hline $\mathrm{H}$ & -5.384956239796 & 0.664727421907 & -0.26284228034 \\
\hline $\mathrm{H}$ & -6.605523874396 & 2.164082416244 & 1.680468390205 \\
\hline $\mathrm{H}$ & -6.522910825193 & 4.732290156127 & -2.728520003242 \\
\hline $\mathrm{F}$ & -7.785138345049 & 5.999688217806 & 0.022894220743 \\
\hline $\mathrm{F}$ & -8.131098860662 & 6.581537011344 & -2.024536478758 \\
\hline $\mathrm{F}$ & -6.150786551038 & 6.779409363921 & -1.16461677692 \\
\hline
\end{tabular}




\begin{tabular}{|c|c|}
\hline & \\
\hline $\mathrm{H}$ & -8.565692172123 \\
\hline $\mathrm{H}$ & -3.840554948435 \\
\hline $\mathrm{H}$ & -7.820314449596 \\
\hline $\mathrm{H}$ & -4.523531112680 \\
\hline $5 a$ & 0297 \\
\hline- & -5.438555527984 \\
\hline $\mathrm{C}$ & -6.065374768501 \\
\hline $\mathrm{C}$ & -6.063470115681 \\
\hline $\mathrm{C}$ & -5.691451388320 \\
\hline $\mathrm{C}$ & -4.787230419981 \\
\hline $\mathrm{C}$ & -7.473991818730 \\
\hline $\mathrm{C}$ & -7.487824335488 \\
\hline 0 & -8.224782907407 \\
\hline $\mathrm{H}$ & -5.493915313061 \\
\hline 0 & -5.028351413569 \\
\hline 0 & -6.800307398531 \\
\hline 0 & -4.687958126670 \\
\hline $\mathrm{H}$ & -7.038199861114 \\
\hline $\mathrm{H}$ & -5.174175980412 \\
\hline $\mathrm{H}$ & -3.773622096463 \\
\hline $\mathrm{H}$ & -7.921843842454 \\
\hline $\mathrm{F}$ & -8.741565263155 \\
\hline $\mathrm{F}$ & -6.724405745904 \\
\hline $\mathrm{F}$ & -7.058467426652 \\
\hline $\mathrm{H}$ & -9.165193723981 \\
\hline $\mathrm{H}$ & -5.225596302699 \\
\hline $\mathrm{H}$ & -7.439283173243 \\
\hline $\mathrm{H}$ & -5.551433050001 \\
\hline & 0053 \\
\hline 0 & -6.321480846935 \\
\hline $\mathrm{C}$ & -5.770006123776 \\
\hline $\mathrm{C}$ & -5.333744734765 \\
\hline $\mathrm{C}$ & -5.898610745142 \\
\hline $\mathrm{C}$ & -5.915460962961 \\
\hline $\mathrm{C}$ & -6.845591090970 \\
\hline $\mathrm{C}$ & -7.290420472134 \\
\hline 0 & -7.981224543111 \\
\hline $\mathrm{H}$ & -4.894840549492 \\
\hline 0 & -3.932442993337 \\
\hline 0 & -7.262902465494 \\
\hline 0 & -4.630305462088 \\
\hline $\mathrm{H}$ & -5.790022883507 \\
\hline $\mathrm{H}$ & -5.293876566345 \\
\hline $\mathrm{H}$ & -6.665889245128 \\
\hline $\mathrm{H}$ & -6.410696736766 \\
\hline $\mathrm{F}$ & -7.991682434036 \\
\hline $\mathrm{F}$ & -8.075442752697 \\
\hline $\mathrm{F}$ & -6.234005895722 \\
\hline $\mathrm{H}$ & -8.020904772381 \\
\hline $\mathrm{H}$ & -3.594808280392 \\
\hline $\mathrm{H}$ & -7.325221869814 \\
\hline $\mathrm{H}$ & -4.646117573007 \\
\hline $6 \mathrm{~b}$ & 0047 \\
\hline 0 & -2.450587442131 \\
\hline $\mathrm{C}$ & -3.586023774960 \\
\hline $\mathrm{C}$ & -3.170272056141 \\
\hline $\mathrm{C}$ & -2.32860228822 \\
\hline
\end{tabular}

3.903242259178

1.638525514640

1.994784038934

2. 982914275136

4.370611535417

3.398880463755

2.064697578983

2.513912756040

3.723049668709

3.857834518716

5.164432596942

4.033973751000

3.262899016704

1.204810674552

2. 925177862626

4.599837214059

1.563281335450

1.722962980411

3. 428691059183

3.102100738290

5.450980781594

5.069454026579

6.215839166620

3. 889180017941

0.883542423758

3.378169566246

4.605680266425

3.691675901806

3.932566475256

2. 551804598687

1.575266160508

2.415968621692

4.598155139397

5.943198297298

3.797975165862

4.587475936293

2.402557344874

1.295220017953

2. 432564330765

2.359453237897

0.669732455485

2.084964048962

4.827374022000

5.834079578774

6.594148319464

6.740739171168

3.119019874880

2.878186648999

0.703621521705

2.942753130273

5.579295437136

5.053472857208

4.069995295018

2. 970802111874
$-2.482368462555$

$-2.091099183835$

$-0.849362567206$

2.165818845981

$-0.168420780137$

$-0.993262321040$

$-0.196554906472$

1. 214440431113

0.933841770342

$-1.352588448784$

$-2.141087720532$

$-0.171906424071$

$-1.920132183645$

$-0.631072910668$

1. 985269815829

1.992823507431

$-0.218560150811$

1.759602783466

0.642278725369

$-2.009227003911$

$-2.545471977146$

$-3.243706875179$

$-1.436231529251$

$-0.340463237857$

$-1.520112279968$

1.405642277096

2.437899438828

0.342355993670

$-0.956124094578$

$-1.517919406465$

$-0.474478071955$

0.795832601994

$-1.809608576527$

$-1.239355780395$

$-1.996954469844$

$-0.881402937496$

$-1.578405934041$

$-0.746893289649$

1. 339413131545

$-2.496284570662$

$-0.384466615361$

1.518995374567

$-2.790333436625$

$-0.103211323816$

$-2.118970677460$

$-0.989147928323$

$-1.300185365094$

$-2.347166942643$

$-1.508202893520$

2.159615212677

0.352912997264

$-0.299592266169$

$-1.394244492383$

$-0.738609489420$ 


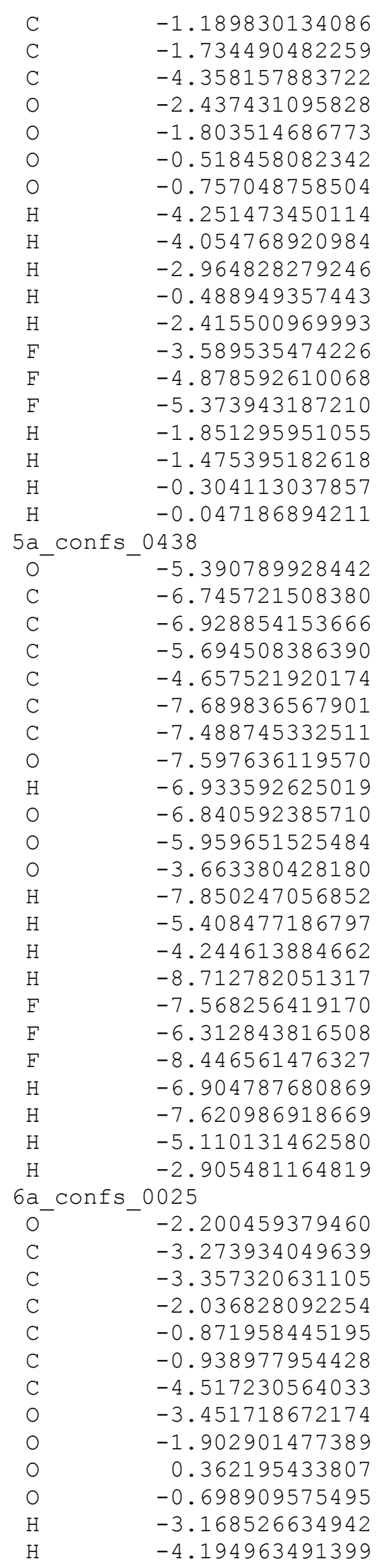

3.563892379759

4.583427177680

6.255045677014

4.689017793364

2.085399197130

2. 552898431278

5. 216150497107

4.542067542384

3.623353089918

2. 370953210965

4.091841509446

4.104751128243

7.088249149426

6.971038761834

5.860211361257

5.367450029212

2. 622810178980

1.848030690744

5.516046493022

3. 614980658198

3.174321789166

2.334887770521

2. 700833201072

2.947193450663

4.373132010069

5.173202039751

5.270286114882

2.530173831042

0.949601640891

3.915693405962

3. 781106762971

2.588491404516

1. 904073778453

2.008620503329

3. 975763927564

4.379463701473

5.811377019854

6.111647865384

4.981143789528

0.661805669619

4.317590982959

3.786608682134

5.839261110662

4.928351546724

4.005768934967

3.231498617720

4.212049461446

5.221537425089

5.771470654992

4.720619540928

2. 376453518756

3.533934650213

4.586837592313

4.314571890289

3.306623673618
0.095848233023

1.092068514973

$-0.823617926248$

$-2.436493377097$

$-1.704583105072$

0.809797270901

1. 829829179969

0.411970118339

$-1.856813931621$

$-0.076653146158$

$-0.574121467695$

1.808551256924

$-1.540152990979$

0.185288301563

$-1.606367402133$

$-2.069162146978$

$-2.442567451405$

0.181959257587

1.244154062812

$-0.749190729278$

$-0.738676889765$

0.555513249343

1. 369190412784

0.274292644149

$-0.817381052888$

$-2.101026509160$

0.253848074304

$-1.606325583373$

0.294871198315

2.049408500882

0.763101222323

1.092286698585

2.060774957210

$-0.114794431043$

$-0.884911485328$

$-3.187031247309$

$-2.155245534812$

$-2.227831393166$

0.877944804952

$-0.195418133530$

2.288869052410

0.165732174394

0.198364720237

0.313641808560

$-0.915696775140$

$-0.937931945067$

$-1.009261282525$

0.141959373101

0.525029838861

$-2.127301468600$

$-2.054229577881$

$-0.871476469663$

1. 371954418308

1. 218229550831

$-0.793779069883$ 


\begin{tabular}{|c|c|c|c|}
\hline & & & \\
\hline $\mathrm{H}$ & -1.958107782571 & 2.657241381444 & -0.004944493077 \\
\hline $\mathrm{H}$ & -0.919723393130 & 4.756702646734 & -1.959816887413 \\
\hline $\mathrm{H}$ & -0.228328112672 & 6.037668901543 & -0.021201227922 \\
\hline $\mathrm{F}$ & -4.780248215251 & 6.586895818262 & -0.511680347009 \\
\hline $\mathrm{F}$ & -4.412556439430 & 6.543264111732 & 1.615295436554 \\
\hline $\mathrm{F}$ & -5.591949882747 & 4.980267469786 & 0.688544222848 \\
\hline $\mathrm{H}$ & -4.339339356172 & 5.083405680698 & -2.233992200387 \\
\hline $\mathrm{H}$ & -2.399500996822 & 1.562983017353 & -1.901805992158 \\
\hline $\mathrm{H}$ & 0.356677077738 & 2.784097654473 & -1.483448182650 \\
\hline $\mathrm{H}$ & -0.019589365235 & 3.909994700259 & 1.225946550074 \\
\hline $5 a_{2}$ & 0040 & & \\
\hline 0 & -6.472722851731 & 3.783502671203 & 0.801715496471 \\
\hline C & -5.768721318932 & 3.959042004807 & -0.431876904126 \\
\hline C & -5.434157427759 & 2.546902917282 & -0.955815672561 \\
\hline C & -5.641848491904 & 1.671046295600 & 0.284496895766 \\
\hline $\mathrm{C}$ & -6.761648861459 & 2.422027907082 & 1.014487936115 \\
\hline C & -6.630008204739 & 4.732161743217 & -1.427143974965 \\
\hline $\mathrm{C}$ & -6.849251046487 & 6.184568901141 & -1.019687367714 \\
\hline 0 & -7.878369631310 & 4.088064875635 & -1.529962643709 \\
\hline $\mathrm{H}$ & -4.847607163197 & 4.518671554556 & -0.234326642301 \\
\hline 0 & -4.174439048698 & 2.450446781947 & -1.574203013105 \\
\hline 0 & -5.952547240263 & 0.330364743726 & 0.013352130373 \\
\hline 0 & -8.020215299109 & 2.060650295919 & 0.497453522452 \\
\hline $\mathrm{H}$ & -6.158069496899 & 2.247346567036 & -1.721721671770 \\
\hline $\mathrm{H}$ & -4.733893362141 & 1.685062855742 & 0.899158311447 \\
\hline $\mathrm{H}$ & -6.789004824456 & 2.237783717437 & 2.089387038107 \\
\hline $\mathrm{H}$ & -6.100116492669 & 4.758589161498 & -2.387813206754 \\
\hline $\mathrm{F}$ & -5.676293474223 & 6.835520044588 & -0.923102146180 \\
\hline $\mathrm{F}$ & -7.482001096159 & 6.315744566698 & 0.152484938831 \\
\hline $\mathrm{F}$ & -7.590825721025 & 6.826449417948 & -1.941876149921 \\
\hline $\mathrm{H}$ & -8.268246024058 & 4.225327746244 & -2.403071053977 \\
\hline $\mathrm{H}$ & -3.477763032272 & 2.598463726530 & -0.920494345700 \\
\hline $\mathrm{H}$ & -6.898459461980 & 0.294941770286 & -0.198344756299 \\
\hline $\mathrm{H}$ & -8.205790428528 & 2.622319733876 & -0.277096720479 \\
\hline 5 b_ & 0042 & & \\
\hline 0 & -6.311889813708 & 3.698566600498 & 0.366665247162 \\
\hline C & -5.772831249065 & 3.919119147035 & -0.940254146565 \\
\hline $\mathrm{C}$ & -5.353221020537 & 2.527879277635 & -1.489678897637 \\
\hline C & -5.900055690026 & 1.568278262828 & -0.428285593067 \\
\hline $\mathrm{C}$ & -5.901501142609 & 2.430663443591 & 0.833361446720 \\
\hline $\mathrm{C}$ & -6.849710151207 & 4.582738506635 & -1.793412778329 \\
\hline $\mathrm{C}$ & -7.300046875020 & 5.924288337904 & -1.218838883639 \\
\hline 0 & -7.983801919417 & 3.782139230518 & -1.987640656135 \\
\hline $\mathrm{H}$ & -4.891977890451 & 4.568120240035 & -0.882508448691 \\
\hline 0 & -3.954231943880 & 2.372758881698 & -1.576758418437 \\
\hline 0 & -7.238374679938 & 1.285310057109 & -0.805745397299 \\
\hline 0 & -4.608031068466 & 2.451198595448 & 1.358191839944 \\
\hline $\mathrm{H}$ & -5.834494262149 & 2.315686579252 & -2.450923526487 \\
\hline $\mathrm{H}$ & -5.291117689075 & 0.665596346310 & -0.336676041050 \\
\hline $\mathrm{H}$ & -6.639207090825 & 2.119456263458 & 1.580036080605 \\
\hline $\mathrm{H}$ & -6.412068376067 & 4.817052314017 & -2.771923231901 \\
\hline $\mathrm{F}$ & -6.245507718564 & 6.718517423040 & -0.948390343245 \\
\hline $\mathrm{F}$ & -8.016424403196 & 5.808243565788 & -0.093395498263 \\
\hline $\mathrm{F}$ & -8.072410223708 & 6.583312650694 & -2.103870845786 \\
\hline $\mathrm{H}$ & -8.014821510979 & 3.082950129024 & -1.310864714783 \\
\hline $\mathrm{H}$ & -3.632900667084 & 2.838546220501 & -2.358389015618 \\
\hline $\mathrm{H}$ & -7.643184904862 & 0.692975784858 & -0.158782406276 \\
\hline
\end{tabular}




\begin{tabular}{|c|c|}
\hline $\mathrm{H}$ & -4.606189709165 \\
\hline \multicolumn{2}{|c|}{$6 a$ confs 1150} \\
\hline 0 & -2.096951637789 \\
\hline $\mathrm{C}$ & -2.880936793541 \\
\hline $\mathrm{C}$ & -3.813391862097 \\
\hline $\mathrm{C}$ & -2.904122472529 \\
\hline $\mathrm{C}$ & -1.970292744784 \\
\hline $\mathrm{C}$ & -1.202792796635 \\
\hline $\mathrm{C}$ & -3.611013565833 \\
\hline 0 & -4.723466420593 \\
\hline 0 & -3.617942015728 \\
\hline 0 & -0.999488496895 \\
\hline 0 & -0.289171034762 \\
\hline $\mathrm{H}$ & -2.242939662553 \\
\hline $\mathrm{H}$ & -4.413246154803 \\
\hline $\mathrm{H}$ & -2.293939257451 \\
\hline $\mathrm{H}$ & -2.561564160217 \\
\hline $\mathrm{H}$ & -0.700337300305 \\
\hline $\mathrm{F}$ & -2.758435490833 \\
\hline $\mathrm{F}$ & -4.531716907799 \\
\hline $\mathrm{F}$ & -4.236914209375 \\
\hline $\mathrm{H}$ & -4.265575389574 \\
\hline $\mathrm{H}$ & -3.994767530463 \\
\hline $\mathrm{H}$ & -1.457687950235 \\
\hline $\mathrm{H}$ & 0.082693854794 \\
\hline \multicolumn{2}{|c|}{$6 a$ confs 0094} \\
\hline 0 & -2.295484247488 \\
\hline $\mathrm{C}$ & -3.372037854486 \\
\hline $\mathrm{C}$ & -3.469313558866 \\
\hline $\mathrm{C}$ & -2.139434816470 \\
\hline $\mathrm{C}$ & -0.973026979977 \\
\hline $\mathrm{C}$ & -1.023239036013 \\
\hline $\mathrm{C}$ & -4.605741923898 \\
\hline 0 & -3.768183188037 \\
\hline 0 & -2.155390574360 \\
\hline 0 & 0.208226797093 \\
\hline 0 & -0.813712562122 \\
\hline $\mathrm{H}$ & -3.247528970387 \\
\hline $\mathrm{H}$ & -4.280362472367 \\
\hline $\mathrm{H}$ & -2.032129366755 \\
\hline $\mathrm{H}$ & -1.064849390382 \\
\hline $\mathrm{H}$ & -0.301225097991 \\
\hline $\mathrm{F}$ & -4.740773674583 \\
\hline $\mathrm{F}$ & -4.565256607707 \\
\hline $\mathrm{F}$ & -5.718800936821 \\
\hline $\mathrm{H}$ & -3.243281569222 \\
\hline $\mathrm{H}$ & -1.267076634600 \\
\hline $\mathrm{H}$ & 0.925176214657 \\
\hline $\mathrm{H}$ & -0.637853549219 \\
\hline \multicolumn{2}{|c|}{ 6b_confs_0942 } \\
\hline $0^{-}$ & -2.045501994186 \\
\hline $\mathrm{C}$ & -3.327734685106 \\
\hline $\mathrm{C}$ & -3.515645415428 \\
\hline $\mathrm{C}$ & -2.386531921616 \\
\hline $\mathrm{C}$ & -1.034585167310 \\
\hline $\mathrm{C}$ & -1.033088943226 \\
\hline $\mathrm{C}$ & -4.31094232245 \\
\hline
\end{tabular}

2.983902142126

5.131408488339

5.457606984760

4.292272513716

3.096691664851

2.804187561203

4.063850361377

6.738812968945

4.022010484706

1.900919310443

1.841528192345

4.442501402500

5.694476498395

4.540856979645

3.358602353992

2. 455633505257

3. 912520394992

7.774884400668

7.039935869872

6.666150255017

4.069281014393

1.915546317266

1.097076059993

3. 633246417324

5.833728071277

4.937742591027

3.936072428378

3.176204594406

4.151415133394

5.186736315493

5.811111455278

4.577584417233

2.271550508048

3.379061405235

4.536153862775

4.401701370056

3.225662371370

2.635732853007

4.690828732835

5.992020910303

6.711536972038

6.499253381492

5.059532504888

5.386760974596

1.893436379665

3.864412051682

5.187860715523

5.772631283030

5.216166318096

3.900568391413

2. 940215967573

3. 625138757556

4. 922911380783

6.299469761134
2.164284228774

$-1.136794858495$

$-0.000715990150$

0.342174618345

0.665385872049

$-0.503053234580$

$-0.917281760898$

$-0.368027608425$

$-0.701562497773$

0.901630826573

$-0.145668707873$

0.075880808297

0.859133032560

1.223032469493

1.541352603195

$-1.361374616689$

$-1.877525539416$

$-0.440900900338$

0.562435122146

$-1.551082212357$

$-1.552849442791$

1.790373490044

0.270037814947

0.460400712135

0.120579916056

0.340015606396

$-0.813529681871$

$-0.848096069216$

$-0.976971155230$

0.153631703792

0.495157498319

$-2.035374509456$

$-1.928279152317$

$-0.961447999529$

1. 364795202470

1.288804044259

$-0.629452140577$

0.104477409067

$-1.928863149745$

$-0.008338628813$

$-0.488802883369$

1. 649466222334

0.514885863588

$-2.114206923692$

$-1.994623465029$

$-1.387448681060$

2.056120973625

$-0.176828928761$

0.006299092712

$-0.751704480663$

$-0.346747387060$

$-0.480257193225$

0.317453604865

$-0.413014608235$ 


\begin{tabular}{|c|c|}
\hline 0 & -3.475147641274 \\
\hline 0 & -2.363747773710 \\
\hline 0 & 0.014162598424 \\
\hline O & 0.156417439891 \\
\hline $\mathrm{H}$ & -3.521431676612 \\
\hline $\mathrm{H}$ & -4.482687813336 \\
\hline $\mathrm{H}$ & -2.524790988582 \\
\hline $\mathrm{H}$ & -0.854856091936 \\
\hline $\mathrm{H}$ & -1.223928628458 \\
\hline $\mathrm{F}$ & -4.053843234891 \\
\hline $\mathrm{F}$ & -4.286193216462 \\
\hline $\mathrm{F}$ & -5.565084511659 \\
\hline $\mathrm{H}$ & -3.338021696148 \\
\hline $\mathrm{H}$ & -2.963967352517 \\
\hline $\mathrm{H}$ & 0.074818915520 \\
\hline $\mathrm{H}$ & 0.881332121076 \\
\hline \multicolumn{2}{|c|}{$6 a$ confs_1062 } \\
\hline 0 & -1.857551935270 \\
\hline $\mathrm{C}$ & -3.147743450497 \\
\hline $\mathrm{C}$ & -3.883121901380 \\
\hline $\mathrm{C}$ & -3.047121165382 \\
\hline $\mathrm{C}$ & -1.632358571689 \\
\hline $\mathrm{C}$ & -1.007031716402 \\
\hline $\mathrm{C}$ & -3.855819745502 \\
\hline 0 & -3.953229232554 \\
\hline 0 & -3.655954551730 \\
\hline 0 & -0.883636673560 \\
\hline 0 & -0.756797538433 \\
\hline $\mathrm{H}$ & -3.091019453117 \\
\hline $\mathrm{H}$ & -4.879628772909 \\
\hline $\mathrm{H}$ & -2.992973320563 \\
\hline $\mathrm{H}$ & -1.666164277376 \\
\hline $\mathrm{H}$ & -0.088834857277 \\
\hline $\mathrm{F}$ & -3.194543413874 \\
\hline $\mathrm{F}$ & -5.087547855371 \\
\hline $\mathrm{F}$ & -4.000915717933 \\
\hline $\mathrm{H}$ & -4.614973613822 \\
\hline $\mathrm{H}$ & -3.063998873707 \\
\hline $\mathrm{H}$ & -0.149250456051 \\
\hline $\mathrm{H}$ & -0.471782905600 \\
\hline \multicolumn{2}{|c|}{ 6b_confs_1018 } \\
\hline 0 & -1.697122591087 \\
\hline $\mathrm{C}$ & -2.873365426298 \\
\hline $\mathrm{C}$ & -3.831993913335 \\
\hline $\mathrm{C}$ & -3.070470474868 \\
\hline $\mathrm{C}$ & -1.762677012832 \\
\hline C & -0.947104992054 \\
\hline $\mathrm{C}$ & -3.481680150875 \\
\hline 0 & -4.257397441481 \\
\hline 0 & -3.851496729292 \\
\hline 0 & -0.959177080255 \\
\hline 0 & 0.216246053614 \\
\hline $\mathrm{H}$ & -2.637527830111 \\
\hline $\mathrm{H}$ & -4.686174517120 \\
\hline $\mathrm{H}$ & -2.840258997268 \\
\hline $\mathrm{H}$ & -1.980486178991 \\
\hline $\mathrm{H}$ & -0.714093280437 \\
\hline
\end{tabular}

4.150079962023

1.821174571935

2.834014442807

5.625694726877

5.027947638632

3. 466829932871

2. 623088981774

3. 877585902465

4.712765239103

6.808981704842

7.325449086047

5.817908368741

3.295848275477

1.137966126345

2. 023192270331

4.983370910142

5.452750109013

5.412425602266

4.113323467995

2. 981954786885

3.042852341333

4.428191826918

6.667465890738

3.914691878305

1.745329239216

2.056058022062

4.629065345358

5.493737191183

4.104573049981

3.132031045669

2.835383398457

4.545125637018

7.774829473784

6.735627707105

6.715167523610

4.499883333160

1.051544085788

1.748108575444

3.780880468712

5.429843707359

5.318355495936

4.250496912559

2. 923662645596

3.121460382270

4.232497719364

6.710385125094

4.525508393468

1. 919358523433

1.960346055305

4.507623928922

5.063317626567

4.142052974447

2. 574019662186

3.424542320455

3. 971373801557
$-2.138372039108$

$-1.216961966479$

0.038117775481

0.157597531141

1.073850594293

$-0.461059888232$

0.695107182446

$-1.534588844780$

1. 384931757598

$-1.621576430997$

0.457124598242

$-0.426572118201$

$-2.574932542444$

$-0.892710723408$

$-0.484508060184$

0.178353074997

$-0.495975113815$

0.077307722144

$-0.296620870684$

0.289865898432

$-0.277740655431$

$-0.043108336138$

$-0.397007417577$

$-1.691631186047$

$-0.001199989012$

0.417610020174

1. 324363551154

1. 170921207862

0.162409583826

1.378376119860

$-1.352122966867$

$-0.628474454023$

$-0.033896456927$

0.137257282735

$-1.733305969557$

$-2.079555912073$

0.321202532803

$-0.128425209490$

1.698750618652

$-0.537896793458$

0.229235616367

$-0.323439789541$

$-0.400603502691$

$-1.163301803729$

$-0.515780915724$

0.266055714378

$-1.644972158206$

$-1.009606972443$

$-1.135306191090$

$-1.226980273201$

1. 274744317074

0.354388744140

0.612415018158

$-2.197885368440$

0.531775894114 


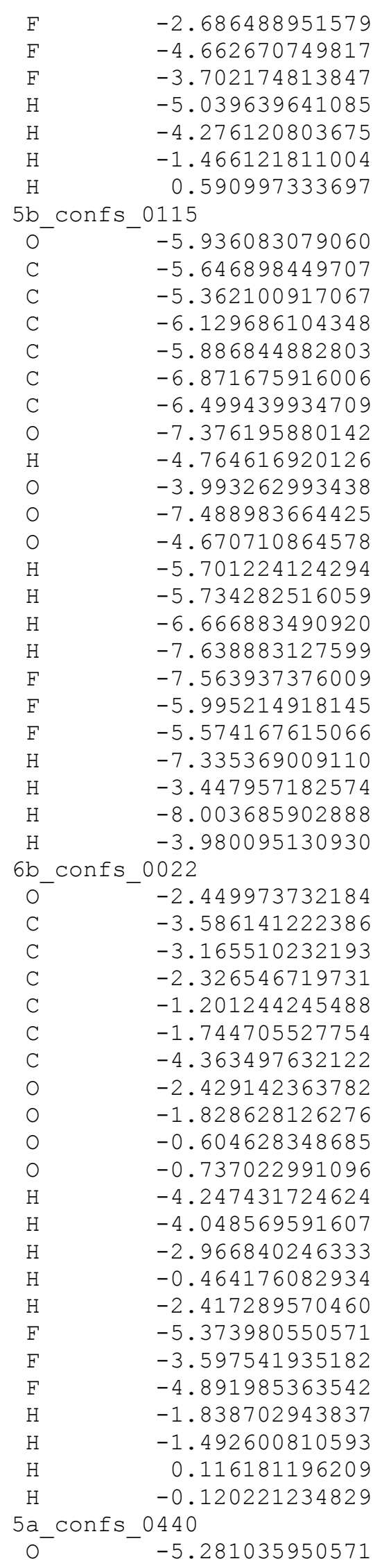

7.573734470072

6.684763442125

7.216114507594

5.090414559122

2. 319107052747

1.230398398978

3.662622294843

3.874627849906

3.839406597590

2. 363713638226

1.636425891240

2. 567739516612

4.434835014501

4.950217356677

5.542500675235

4.463268326029

2.050220960673

1. 626873342634

2. 329914947495

2.067772919935

0.631279640578

2.510813778314

3.661794428563

5. 451022741602

3.962119483606

5.921958256321

5.317376208082

2.503340634094

1.140948672471

2.251829119617

5.593261077479

5.053651675262

4.056821277065

2.944416837529

3. 536882520372

4.598939444644

6.244651465100

4. 672424922075

2.078137554674

2. 487515868540

5.218232747550

4.552058019627

3.609951505891

2. 341533226598

4.020949949948

4.138173247339

5.834961583983

7.075087648480

6.967981978735

5.346567862130

2.631364930340

2.850127059134

5.645207597506

4.023648932639
0.914650106220

0.908802631343

$-0.955652580109$

$-1.641680642372$

$-1.784704354608$

$-1.516768236203$

$-1.516488459978$

0.275129106530

$-1.127333198268$

$-1.456441947274$

$-0.354785608371$

0.841560828410

$-1.848450824075$

$-3.227322095858$

$-1.148427051140$

$-1.313427289654$

$-1.249377695017$

$-0.733535515484$

1.479464399513

$-2.453194945488$

$-0.180020175368$

1. 603246263794

$-1.983962632824$

$-3.871994670261$

$-3.991523706021$

$-3.176035068848$

$-0.206047044642$

$-1.905349357803$

$-0.076998496842$

0.800526724991

0.342772834879

$-0.298869965243$

$-1.376846525564$

$-0.728779673491$

0.120744261282

1.080910104967

$-0.839671321717$

$-2.419806033112$

$-1.721587970570$

0.847129232140

1. 799420688975

0.423238530570

$-1.841619024875$

$-0.073699723166$

$-0.542410289681$

1.817089762919

$-1.621217074036$

$-1.561645099508$

0.160173228433

$-2.051336805475$

$-2.443253010484$

1.379572133961

1.186191738796

$-0.377278863948$ 


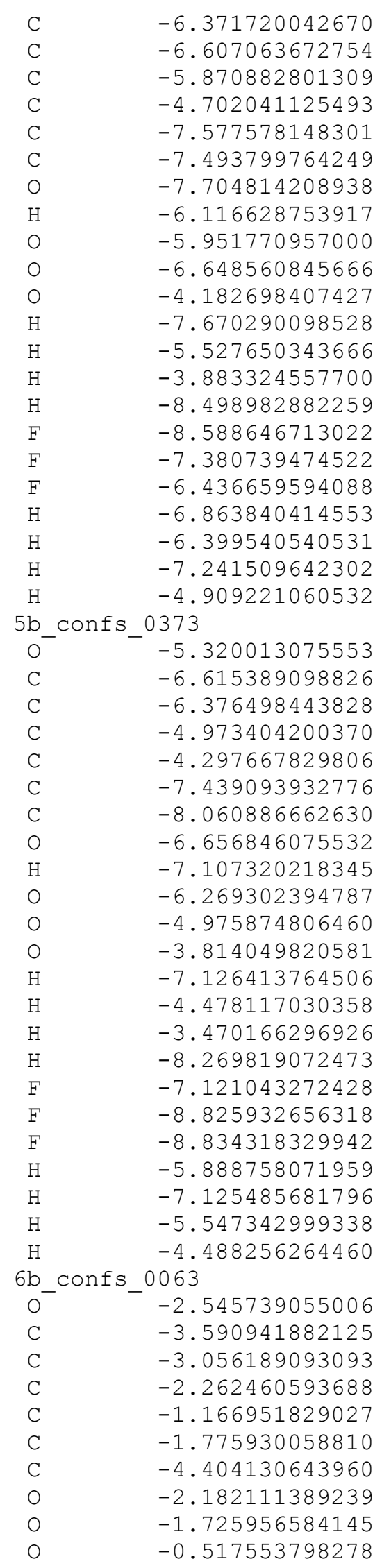

3.253256261117

2. 113741431980

2.609912385429

3.396274663099

4.192707159479

5.025641676397

5.056464067939

2.810686159004

0.927557524158

3. 461209701141

4.358773610238

1.937090343849

1.777792160526

2.744677051730

3.608044269534

5.783817141254

4.244302527541

5.855915509940

5.538572333909

0.555480948103

4.003342669954

4. 679091471041

4.192388008014

3. 587604517533

2.078592010557

2.047308024015

3.200945018004

4.277559868892

5.581573133422

4.547864404117

3.732101781564

1.392117732586

2. 238513682149

2.823892758801

1.618992768918

1.096172303002

3.650272720464

3.636528406482

6.483428247086

6.142682777245

5.382103054019

5.064081742428

1.376290770050

2.989441367027

2. 282544903624

5.592234739033

5.051353305132

4.162861470875

3.045042961121

3.645585695322

4.593646100802

6.235726495174

4.866276543431

2.195320996588

2. 560469041082
$-0.869375055119$

0.157154874530

1.396800659264

0.782637179940

$-1.028228145512$

$-2.302689022618$

0.075559260967

$-1.839349870083$

$-0.244821674851$

2.213720571536

1. 620275510791

0.357171787990

2.013037386461

0.463375846001

$-1.104259822446$

$-2.464029369307$

$-3.388907989099$

$-2.288074586988$

0.158462454581

$-1.015299278145$

1. 664709089424

2.180409056629

$-1.221398551422$

$-1.204739563536$

$-0.969046413557$

$-0.354115675419$

$-1.112453250204$

$-0.101116845287$

$-0.589180234777$

1.036425798296

$-2.173054675362$

$-2.207888631765$

1.039267985598

$-2.364318629005$

$-0.317225315558$

$-0.556656623770$

$-0.562061812417$

0.207591031884

$-0.924614597986$

0.358443224322

$-1.668604294999$

0.736422991520

$-2.655165678431$

1. 273443017187

$-2.806953255309$

0.468241253557

$-0.306816036499$

$-1.445712855551$

$-0.777988462006$

0.093787811635

1.128747994062

$-0.799760256873$

$-2.300335348472$

$-1.763618062685$

0.724254026047 


\begin{tabular}{|c|c|}
\hline & \\
\hline 0 & -0.812566614484 \\
\hline $\mathrm{H}$ & -4.277298557111 \\
\hline $\mathrm{H}$ & -3.897218334082 \\
\hline $\mathrm{H}$ & -2.949131439622 \\
\hline $\mathrm{H}$ & -0.471937617475 \\
\hline $\mathrm{H}$ & -2.412078976610 \\
\hline $\mathrm{F}$ & -5.406073864227 \\
\hline $\mathrm{F}$ & -3.676277569796 \\
\hline $\mathrm{F}$ & -4.951689992760 \\
\hline $\mathrm{H}$ & -2.686160728109 \\
\hline $\mathrm{H}$ & -1.135706989886 \\
\hline $\mathrm{H}$ & 0.286701704995 \\
\hline $\mathrm{H}$ & -0.228296093462 \\
\hline $6 a$ & 0043 \\
\hline 0 & -2.356008097043 \\
\hline $\mathrm{C}$ & -3.439000473067 \\
\hline $\mathrm{C}$ & -3.521138244101 \\
\hline $\mathrm{C}$ & -2.208843645958 \\
\hline $\mathrm{C}$ & -1.012650384867 \\
\hline $\mathrm{C}$ & -1.094330842888 \\
\hline $\mathrm{C}$ & -4.669018215505 \\
\hline 0 & -3.760030886476 \\
\hline 0 & -2.210480106795 \\
\hline 0 & 0.140483223310 \\
\hline 0 & -0.898330830489 \\
\hline $\mathrm{H}$ & -3.327755572660 \\
\hline $\mathrm{H}$ & -4.352197870081 \\
\hline $\mathrm{H}$ & -2.149696659252 \\
\hline $\mathrm{H}$ & -0.989413172760 \\
\hline $\mathrm{H}$ & -0.376195914500 \\
\hline $\mathrm{F}$ & -4.785010784107 \\
\hline $\mathrm{F}$ & -4.639108120887 \\
\hline $\mathrm{F}$ & -5.785907831729 \\
\hline $\mathrm{H}$ & -3.225716460294 \\
\hline $\mathrm{H}$ & -1.358015390204 \\
\hline $\mathrm{H}$ & 0.910647543717 \\
\hline $\mathrm{H}$ & -0.238781263363 \\
\hline $6 \mathrm{~b}_{2}$ & 0023 \\
\hline O & -2.433794655731 \\
\hline C & -3.545132989672 \\
\hline C & -3.090166328808 \\
\hline $\mathrm{C}$ & -2.254794900655 \\
\hline $\mathrm{C}$ & -1.155323844471 \\
\hline C & -1.752958854876 \\
\hline $\mathrm{C}$ & -4.306023840644 \\
\hline O & -2.335704812261 \\
\hline O & -1.696438702356 \\
\hline 0 & -0.485325217303 \\
\hline 0 & -0.774563780426 \\
\hline $\mathrm{H}$ & -4.229328779370 \\
\hline $\mathrm{H}$ & -3.960258795809 \\
\hline $\mathrm{H}$ & -2.901936519341 \\
\hline $\mathrm{H}$ & -0.439652812615 \\
\hline $\mathrm{H}$ & -2.469631051577 \\
\hline $\mathrm{F}$ & -3.517798981887 \\
\hline $\mathrm{F}$ & -4.868032923119 \\
\hline $\mathrm{F}$ & -5.289171233503 \\
\hline
\end{tabular}

5.228590773367

4.457543862992

3. 728015322513

2. 482304023518

4.211546740721

4.032205814563

5.814030981599

7.110993290635

6.913153673684

5.368616067555

1.573407155538

2.874400350615

5.735774594139

5.826766804229

4. 928929988627

3.968209223780

3.182088221379

4.127227682190

5.195862382597

5.806302633842

4.654740841503

2.295700400580

3.322613621317

4.621568537678

4.365297273739

3.269854364600

2. 620865737427

4.619206686513

6.002541827764

6.735134147421

6.460121774574

5.060957940107

5.461273335753

1.838867948538

3.738492364802

3.916576261037

5.561687268831

5.012486156579

4.008163717893

2. 919926368142

3.551393885047

4. 576757043574

6.198255637169

4. 615107857352

2.035178224214

2. 597122026241

5.263537190221

4.512411049301

3.549045072758

2.319689931621

4.080715714702

4.091161899367

7.023982085569

6.928134805236

5.780015917176
1.894209685712

0.316974574351

$-1.998839380488$

$-0.125738431639$

$-0.543038212407$

1. 827514361717

$-1.591260095402$

$-1.510468185396$

0.219677965473

$-2.951626948078$

$-1.315024047957$

1.156940467824

1.311078183073

0.141460819736

0.321653835330

$-0.867029812031$

$-0.877077171543$

$-0.943632123000$

0.159274436622

0.486271953184

$-2.077116813694$

$-1.972056772344$

$-0.754609952949$

1.422338865991

1.255542379639

$-0.733814231235$

0.068082817768

$-1.923753634935$

$-0.021631375895$

$-0.473290140342$

1.659895861842

0.469237585479

$-2.103708202059$

$-1.977444928279$

$-1.162810564481$

1.327817167197

0.391584687198

$-0.285713792402$

$-1.347593042177$

$-0.657946069860$

0.193948890805

1.148524566328

$-0.860260355736$

$-2.381885030286$

$-1.604612121631$

0.988475429576

1.857123826112

0.415692236811

$-1.824933933250$

$-0.007397386616$

$-0.454819405112$

1.832970427529

$-1.563641342598$

0.115708757817

$-1.671396342979$ 


\begin{tabular}{|c|c|c|c|}
\hline $\mathrm{H}$ & -1.763061851624 & 5.304295075136 & -2.013296848242 \\
\hline $\mathrm{H}$ & -1.358806417508 & 2.572180360516 & -2.338480658649 \\
\hline $\mathrm{H}$ & -0.097608130775 & 1.929850672866 & 0.405656859141 \\
\hline $\mathrm{H}$ & -0.098584575668 & 4.623502040491 & 2.125090648220 \\
\hline \multicolumn{4}{|c|}{ 5b confs 0110} \\
\hline $0^{-}$ & -6.267772648143 & 3.699036810264 & 0.442643657288 \\
\hline $\mathrm{C}$ & -5.763683397216 & 3.908181553764 & -0.877207980746 \\
\hline $\mathrm{C}$ & -5.339170701832 & 2.516690013485 & -1.421149591957 \\
\hline $\mathrm{C}$ & -5.866789963793 & 1.556470972532 & -0.344139583020 \\
\hline $\mathrm{C}$ & -5.850660602208 & 2.430222259653 & 0.908849024291 \\
\hline $\mathrm{C}$ & -6.865695335325 & 4.535394125331 & -1.722550193159 \\
\hline $\mathrm{C}$ & -7.282728353319 & 5.919231081597 & -1.234265501022 \\
\hline 0 & -7.991733709067 & 3.687414807062 & -1.703937310358 \\
\hline $\mathrm{H}$ & -4.892834248351 & 4.573387831138 & -0.852810346293 \\
\hline 0 & -3.937643644873 & 2.378949932783 & -1.524954101994 \\
\hline 0 & -7.180709570684 & 1.128614762602 & -0.628576205482 \\
\hline 0 & -4.549796198094 & 2.463701381596 & 1.420855125725 \\
\hline $\mathrm{H}$ & -5.824697235310 & 2.295373703008 & -2.378736237099 \\
\hline $\mathrm{H}$ & -5.231803919094 & 0.674874006417 & -0.247225614215 \\
\hline $\mathrm{H}$ & -6.579422247534 & 2.116374585800 & 1.661586175162 \\
\hline $\mathrm{H}$ & -6.478986797284 & 4.679232394659 & -2.738643947130 \\
\hline $\mathrm{F}$ & -6.227572646422 & 6.751854713546 & -1.190257096199 \\
\hline $\mathrm{F}$ & -7.844134837470 & 5.910514232270 & -0.020447875509 \\
\hline $\mathrm{F}$ & -8.183760656718 & 6.459101468706 & -2.078153745915 \\
\hline $\mathrm{H}$ & -8.495046907106 & 3.774564606972 & -2.523816471557 \\
\hline $\mathrm{H}$ & -3.629287183696 & 2.852704669308 & -2.307208422353 \\
\hline $\mathrm{H}$ & -7.705854440474 & 1.902717226279 & -0.894864540414 \\
\hline $\mathrm{H}$ & -4.543814755989 & 3.006292861228 & 2.220110781956 \\
\hline \multicolumn{4}{|c|}{ 5b_confs_0093 } \\
\hline 0 & -6.024638404457 & 3.853644459229 & 0.275927717714 \\
\hline $\mathrm{C}$ & -5.708735778462 & 3.864598364048 & -1.122600762979 \\
\hline $\mathrm{C}$ & -5.348525280984 & 2.418759146309 & -1.485558435831 \\
\hline C & -6.055567662964 & 1.621101018201 & -0.378252293371 \\
\hline C & -5.861935912122 & 2.548913395211 & 0.821880186012 \\
\hline $\mathrm{C}$ & -6.950015045345 & 4.425687353719 & -1.837173860895 \\
\hline $\mathrm{C}$ & -6.610088684168 & 4.947615810386 & -3.222547673313 \\
\hline 0 & -7.471800401009 & 5.525399001157 & -1.137237050813 \\
\hline $\mathrm{H}$ & -4.847916277807 & 4.522441007601 & -1.285493518847 \\
\hline 0 & -3.937534127656 & 2.303477692161 & -1.353984936511 \\
\hline 0 & -7.445017526606 & 1.537489635930 & -0.616774743167 \\
\hline 0 & -4.617997752533 & 2.400043061805 & 1.432887396815 \\
\hline $\mathrm{H}$ & -5.683592577776 & 2.132731526862 & -2.487133931417 \\
\hline $\mathrm{H}$ & -5.601178939888 & 0.639291651923 & -0.211377574962 \\
\hline $\mathrm{H}$ & -6.623751306156 & 2.416202500226 & 1.590632635210 \\
\hline $\mathrm{H}$ & -7.700511321194 & 3.633552663898 & -1.958233309391 \\
\hline $\mathrm{F}$ & -7.695972777800 & 5.429432607370 & -3.84578724223 \\
\hline $\mathrm{F}$ & -6.101453786134 & 3.972457584525 & -3.999428477085 \\
\hline $\mathrm{F}$ & -5.702793497475 & 5.936616410539 & -3.184668466637 \\
\hline $\mathrm{H}$ & -7.461586935753 & 5.288008697548 & -0.197291141643 \\
\hline $\mathrm{H}$ & -3.651409071676 & 1.443827674352 & -1.689960230940 \\
\hline $\mathrm{H}$ & -7.616082312102 & 0.870739588854 & -1.294045303806 \\
\hline & -3.934794619933 & 2.391869148147 & 0.74252101809 \\
\hline \multicolumn{4}{|c|}{ 5b_confs_1155 } \\
\hline 0 & -6.103182088957 & 3.415868568617 & -1.781683897901 \\
\hline $\mathrm{C}$ & -6.651892818536 & 3.607556527750 & -0.47546789807 \\
\hline $\mathrm{C}$ & -5.782719597353 & 2.786638288587 & 0.5029052145 \\
\hline $\mathrm{C}$ & -4.611975590542 & 2.312359285886 & -0.37774758835 \\
\hline
\end{tabular}




\begin{tabular}{|c|c|}
\hline$C$ & \\
\hline $\mathrm{C}$ & -6.591499298970 \\
\hline $\mathrm{C}$ & -7.646822383592 \\
\hline 0 & -5.318546423571 \\
\hline $\mathrm{H}$ & -7.681838705662 \\
\hline 0 & -6.582962223996 \\
\hline 0 & -3.526012268384 \\
\hline O & -5.937790564531 \\
\hline $\mathrm{H}$ & -5.394474614041 \\
\hline $\mathrm{H}$ & -4.247725919272 \\
\hline $\mathrm{H}$ & -4.508111605627 \\
\hline $\mathrm{H}$ & -6.781437451356 \\
\hline $\mathrm{F}$ & -8.877552055476 \\
\hline $\mathrm{F}$ & -7.413523362405 \\
\hline $\mathrm{F}$ & -7.665402236798 \\
\hline $\mathrm{H}$ & -5.170163003851 \\
\hline $\mathrm{H}$ & -6.092623749043 \\
\hline $\mathrm{H}$ & -3.863228202521 \\
\hline $\mathrm{H}$ & -6.308487094954 \\
\hline \multicolumn{2}{|c|}{ 5b confs 0059} \\
\hline $0^{-}$ & -5.993770874586 \\
\hline $\mathrm{C}$ & -5.705441682797 \\
\hline $\mathrm{C}$ & -5.326357825727 \\
\hline $\mathrm{C}$ & -6.030315233165 \\
\hline $\mathrm{C}$ & -5.842668336634 \\
\hline $\mathrm{C}$ & -6.968809466290 \\
\hline $\mathrm{C}$ & -6.650446303800 \\
\hline 0 & -7.516879584202 \\
\hline $\mathrm{H}$ & -4.866219340368 \\
\hline 0 & -3.937055107072 \\
\hline 0 & -7.418240126913 \\
\hline 0 & -4.606767416708 \\
\hline $\mathrm{H}$ & -5.652526804633 \\
\hline $\mathrm{H}$ & -5.563545420455 \\
\hline $\mathrm{H}$ & -6.612912711600 \\
\hline $\mathrm{H}$ & -7.698176631968 \\
\hline $\mathrm{F}$ & -7.752325824055 \\
\hline $\mathrm{F}$ & -6.111539771982 \\
\hline $\mathrm{F}$ & -5.775413222592 \\
\hline $\mathrm{H}$ & -7.472622001160 \\
\hline $\mathrm{H}$ & -3.430602220933 \\
\hline $\mathrm{H}$ & -7.583478130990 \\
\hline $\mathrm{H}$ & -3.917085961368 \\
\hline \multicolumn{2}{|c|}{$5 a$ confs 0783} \\
\hline 0 & -5.656466916050 \\
\hline $\mathrm{C}$ & -5.533512637265 \\
\hline $\mathrm{C}$ & -4.943936831350 \\
\hline $\mathrm{C}$ & -5.491635749209 \\
\hline $\mathrm{C}$ & -5.946727560201 \\
\hline $\mathrm{C}$ & -6.871253930925 \\
\hline $\mathrm{C}$ & -7.459684840440 \\
\hline 0 & -7.840815363670 \\
\hline $\mathrm{H}$ & -4.839333161416 \\
\hline 0 & -3.534480848093 \\
\hline 0 & -6.484889297966 \\
\hline 0 & -7.323801768269 \\
\hline $\mathrm{H}$ & -5.250116314372 \\
\hline
\end{tabular}

2.293921806661

5.110188239594

5.901741850210

5.627442114387

3.239306944091

1.738810306598

3.213153872595

1.098228033585

3.406487985509

1.325995530044

2. 455852850520

5.292381397746

5.403205959939

5.895231076524

7.187157325429

5.472572859504

1.270567622512

4.122190205707

1.068141348008

3.878347502551

3.893682668797

2.447791233633

1.646369805858

2. 568076688540

4.429350920525

4.973464299247

5.506048705600

4.577899464419

2.221237733641

1.553319251684

2. 410504297217

2.156050956031

0.669521072809

2.432731337418

3. 618724940104

5.424289112739

4.020524283292

5.991039024604

5.267646171080

2.707727793466

0.885819630007

2.394433106741

3.925157600095

4.172207407106

2.874617637487

1.778863520062

2.561147815543

4.538841590232

5.829467586200

3.523751835095

5.008064719574

2. 861678041111

0.976308793540

2.364418854487

2.682665610009
$-1.773613901221$

$-0.189719842389$

$-0.955877458588$

$-0.496632213346$

$-0.425874771812$

1.006166466173

$-0.321187172809$

$-1.932936182847$

1.321357223369

$-0.083887989475$

$-2.574567288474$

0.871830228397

$-0.758189385403$

$-2.280305617946$

$-0.568992038392$

$-1.444679782408$

1.693621494872

$-0.278848837084$

$-2.824668760839$

0.264862232157

$-1.138670885834$

$-1.502588846042$

$-0.402056473493$

0.804445714711

$-1.837439364503$

$-3.219025281302$

$-1.122095136546$

$-1.312170488813$

$-1.318712724825$

$-0.640775108755$

1. 427726435017

$-2.505616914349$

$-0.246086520017$

1. 564262027404

$-1.963266949898$

$-3.837382603285$

$-4.003386354026$

$-3.175622808414$

$-0.183270516987$

$-1.981827637285$

$-1.318642726170$

0.743940931254

0.646324641106

$-0.752182891727$

$-1.318197961760$

$-0.381730634230$

0.889616207509

$-1.403541304371$

$-0.839481731586$

$-1.310365738737$

$-0.875983601552$

$-1.186497359629$

$-0.962244183139$

1.096329380895

$-2.352869085830$ 


\begin{tabular}{|c|c|}
\hline & \\
\hline $\mathrm{H}$ & -4.668296885178 \\
\hline $\mathrm{H}$ & -5.393006197984 \\
\hline $\mathrm{H}$ & -6.692221899044 \\
\hline $\mathrm{F}$ & -6.544781212034 \\
\hline $\mathrm{F}$ & -7.954590557447 \\
\hline $\mathrm{F}$ & -8.471985097201 \\
\hline $\mathrm{H}$ & -8.009470616499 \\
\hline $\mathrm{H}$ & -3.149440328915 \\
\hline $\mathrm{H}$ & -7.205226928637 \\
\hline $\mathrm{H}$ & -7.560325057835 \\
\hline & 0015 \\
\hline 0 & -2.538374116700 \\
\hline $\mathrm{C}$ & -3.585851406448 \\
\hline $\mathrm{C}$ & -3.026850688691 \\
\hline $\mathrm{C}$ & -2.339694251626 \\
\hline $\mathrm{C}$ & -1.298829420683 \\
\hline C & -1.929147170837 \\
\hline $\mathrm{C}$ & -4.282297626543 \\
\hline 0 & -2.161591246174 \\
\hline 0 & -1.754327630011 \\
\hline 0 & -0.808036961950 \\
\hline 0 & -1.006586989975 \\
\hline $\mathrm{H}$ & -4.338548764828 \\
\hline $\mathrm{H}$ & -3.841192648799 \\
\hline $\mathrm{H}$ & -3.107244504241 \\
\hline $\mathrm{H}$ & -0.488165518709 \\
\hline $\mathrm{H}$ & -2.684664637487 \\
\hline $\mathrm{F}$ & -5.187229356527 \\
\hline $\mathrm{F}$ & -3.433980727740 \\
\hline $\mathrm{F}$ & -4.934510393873 \\
\hline $\mathrm{H}$ & -1.569745054750 \\
\hline $\mathrm{H}$ & -1.279630619769 \\
\hline $\mathrm{H}$ & -0.028391158441 \\
\hline $\mathrm{H}$ & -0.311109105196 \\
\hline $5 a_{-}$ & 0021 \\
\hline 0 & -5.445586709946 \\
\hline $\mathrm{C}$ & -6.660592952055 \\
\hline $\mathrm{C}$ & -6.214502016544 \\
\hline C & -4.892956923503 \\
\hline $\mathrm{C}$ & -4.450868739818 \\
\hline $\mathrm{C}$ & -7.585172938100 \\
\hline $\mathrm{C}$ & -8.049033521522 \\
\hline 0 & -7.017706774682 \\
\hline $\mathrm{H}$ & -7.166849431843 \\
\hline 0 & -6.001718113981 \\
\hline 0 & -5.010178024865 \\
\hline 0 & -4.350311507062 \\
\hline $\mathrm{H}$ & -6.941479171640 \\
\hline $\mathrm{H}$ & -4.149626924813 \\
\hline $\mathrm{H}$ & -3.508495280544 \\
\hline $\mathrm{H}$ & -8.489585926168 \\
\hline $\mathrm{F}$ & -8.490452261081 \\
\hline $\mathrm{F}$ & -7.065764176908 \\
\hline $\mathrm{F}$ & -9.056421865725 \\
\hline $\mathrm{H}$ & -6.194133428851 \\
\hline $\mathrm{H}$ & -5.731057816079 \\
\hline $\mathrm{H}$ & -5.704576670645 \\
\hline
\end{tabular}

1.111366893300

2.278566739097

4.724173580524

6.811301721266

5.657317514725

6.273810308307

3.329911862572

3. 508674269268

1.548488990167

2. 700197110234

5.456023826306

5.018612414665

4.150290890593

2.952009986875

3. 416327242040

4.376542014919

6.281473256499

4.884283668016

2.151525086767

2. 254312297754

4.902176981876

4.451839298753

3.788076012131

2.380005683704

3. 942863643972

3.855783955499

5.988091774398

7.184513986969

6.884325126629

5.429361078682

1.434502577503

2.476236605907

5. 361622589543

3. 615246311607

3.057114835605

2. 016575780117

2.590946552394

3.631882122198

4.120135992191

5.139530664556

4.810486643204

2.569421543747

0.819037479492

3.106621887819

4.904052091763

1.872243242346

1.792701797001

3. 371457855196

3.621980789421

4.545364257897

5.986743784655

5.889707980667

5.248805336117

0.122070827426

3.783547186251
$-0.121488787943$

1. 787055676552

$-2.467711766873$

$-0.779063602816$

0.398209972166

$-1.603495691839$

$-0.366291287309$

$-1.791205040273$

$-1.268452306296$

1. 971267097678

0.506941575056

$-0.335939653135$

$-1.468636218139$

$-0.808091954306$

0.204712323789

1.214987284299

$-0.820398857510$

$-2.306601470575$

$-1.806940900905$

0.837516964259

2.100325030832

0.231336911784

$-2.102318468059$

$-0.262123543633$

$-0.323381508571$

1.818656395553

$-1.767189117180$

$-1.329280841419$

0.188236029519

$-1.768528446264$

$-1.363778906715$

1. 362843302937

1.606654068382

$-1.470156613736$

$-0.974996144866$

0.050560018824

0.613939962368

$-0.462301142642$

$-0.374519616389$

$-1.411955410263$

0.712192570893

$-1.811618217841$

$-0.673963964125$

1. 913606345222

0.127397795488

0.858328180237

0.668895503355

$-0.948016943711$

$-0.010724993080$

$-2.532867552614$

$-1.760471334463$

$-0.934423624347$

0.418189536002

$-0.062646804647$

1. 916769072902 


\begin{tabular}{|c|c|}
\hline \multirow{2}{*}{\multicolumn{2}{|c|}{$\begin{array}{rr}\mathrm{H} & -3.90 \\
6 \mathrm{~b} & \text { confs } 0082\end{array}$}} \\
\hline & \\
\hline 0 & -2.492287495697 \\
\hline $\mathrm{C}$ & -3.583714027919 \\
\hline $\mathrm{C}$ & -3.063790870461 \\
\hline $\mathrm{C}$ & -2.247075676538 \\
\hline $\mathrm{C}$ & -1.154973820910 \\
\hline $\mathrm{C}$ & -1.754516993350 \\
\hline $\mathrm{C}$ & -4.406943828025 \\
\hline 0 & -2.312042965105 \\
\hline 0 & -1.583273592980 \\
\hline 0 & -0.521431279174 \\
\hline 0 & -0.730502846815 \\
\hline $\mathrm{H}$ & -4.241119027470 \\
\hline $\mathrm{H}$ & -3.902102213977 \\
\hline $\mathrm{H}$ & -2.926502025377 \\
\hline $\mathrm{H}$ & -0.423326742511 \\
\hline $\mathrm{H}$ & -2.440588995348 \\
\hline $\mathrm{F}$ & -5.002210681911 \\
\hline $\mathrm{F}$ & -5.374635963920 \\
\hline $\mathrm{F}$ & -3.673843237074 \\
\hline $\mathrm{H}$ & -1.717285992400 \\
\hline $\mathrm{H}$ & -2.210305297323 \\
\hline $\mathrm{H}$ & -0.286080134441 \\
\hline $\mathrm{H}$ & -1.087946291276 \\
\hline \multicolumn{2}{|c|}{ 6b confs 0105} \\
\hline 0 & -2.493949712864 \\
\hline $\mathrm{C}$ & -3.549956721692 \\
\hline $\mathrm{C}$ & -3.019053038551 \\
\hline $\mathrm{C}$ & -2.210179662098 \\
\hline $\mathrm{C}$ & -1.111407373205 \\
\hline $\mathrm{C}$ & -1.718150171418 \\
\hline $\mathrm{C}$ & -4.386496545468 \\
\hline 0 & -2.149311794439 \\
\hline 0 & -1.559407778454 \\
\hline 0 & -0.441453703358 \\
\hline 0 & -0.695715413577 \\
\hline $\mathrm{H}$ & -4.219327247104 \\
\hline $\mathrm{H}$ & -3.863989440034 \\
\hline $\mathrm{H}$ & -2.887083767194 \\
\hline $\mathrm{H}$ & -0.415429732364 \\
\hline $\mathrm{H}$ & -2.374355576481 \\
\hline $\mathrm{F}$ & -5.415032987382 \\
\hline $\mathrm{F}$ & -3.687069361371 \\
\hline $\mathrm{F}$ & -4.897338856226 \\
\hline $\mathrm{H}$ & -2.651288393312 \\
\hline $\mathrm{H}$ & -2.189385689942 \\
\hline $\mathrm{H}$ & -0.214832401423 \\
\hline$\Pi$ & -1.053084632042 \\
\hline
\end{tabular}

5.501325038330

5.584247792178

5.048518850723

4.174544521537

3.048862681200

3.608533042247

4.579415062530

6.242095857542

4.926190777566

2.230064196248

2.568400711359

5.205578227309

4.456071166643

3.741950640630

2.444558212001

4.164671128179

4.054018822222

6.843779332744

5.851653522425

7.176085864403

5.523958802575

1. 617359678141

1.886266551029

5.666474558565

5.573893441750

5.042483674258

4.187045268112

3.059990858154

3.634700866711

4.566148239681

6.225565172733

4.905831376117

2.230099567537

2. 596222889752

5.195962205676

4.424907405806

3.763207624633

2.464028896175

4.220437457949

3.999961445091

5.804710364344

7.105996993880

6.898080691223

5.505551522732

1.603674719735

1.926124698703

5.630974619246
$-0.491216622568$

0.391757269909

$-0.326404386409$

$-1.473073067103$

$-0.820097778827$

0.087638766200

1.095638255507

$-0.785855589989$

$-2.399507472400$

$-1.759786591259$

0.796698068586

1.774489041616

0.327328791561

$-2.027656165553$

$-0.200456732429$

$-0.519026434664$

1. 778496152789

0.258423551487

$-1.630455837830$

$-1.406054306725$

$-1.923781381446$

$-2.163432467004$

0.151619415621

2.544798898362

0.494005824903

$-0.268361573629$

$-1.433950415236$

$-0.788449741012$

0.101445793807

1.142581689440

$-0.721948089713$

$-2.278141663361$

$-1.727157111446$

0.782126319986

1.824457705292

0.351790993267

$-1.992107404692$

$-0.159221807158$

$-0.515106947529$

1.823649344615

$-1.478970802662$

$-1.457794243784$

0.318500487954

$-2.843177681351$

$-2.104115119304$

0.121509491796

2.609534949819 